DiRECTEUR DE LA PUBLICATION: Bruno David,

Président du Muséum national d'Histoire naturelle

RÉdACTEUR EN CHEF / EDITOR-IN-CHIEF: Didier Merle

ASSISTANTS DE RÉDACTION / AsSISTANT EDITORS: Emmanuel Côtez (geodiv@mnhn.fr); Anne Mabille

MISE EN PAGE / PAGE LAYOUT: Emmanuel Côtez

COMITÉ SCIENTIFIQUE / SCIENTIFIC BOARD:

Christine Argot (MNHN, Paris)

Beatrix Azanza (Museo Nacional de Ciencias Naturales, Madrid)

Raymond L. Bernor (Howard University, Washington DC)

Alain Blieck (chercheur CNRS retraité, Haubourdin)

Henning Blom (Uppsala University)

Jean Broutin (UPMC, Paris)

Gaël Clément (MNHN, Paris)

Ted Daeschler (Academy of Natural Sciences, Philadelphie)

Bruno David (MNHN, Paris)

Gregory D. Edgecombe (The Natural History Museum, Londres)

Ursula Göhlich (Natural History Museum Vienna)

Jin Meng (American Museum of Natural History, New York)

Brigitte Meyer-Berthaud (CIRAD, Montpellier)

Zhu Min (Chinese Academy of Sciences, Pékin)

Isabelle Rouget (UPMC, Paris)

Sevket Sen (MNHN, Paris)

Stanislav Štamberg (Museum of Eastern Bohemia, Hradec Králové)

Paul Taylor (The Natural History Museum, Londres)

COUVERTURE / COVER:

Réalisée d'après les figures de l'article/Created from article figures.

Geodiversitas est indexé dans / Geodiversitas is indexed in:

- Science Citation Index Expanded (SciSearch ${ }^{\circledR}$ )

- ISI Alerting Services ${ }^{\circledR}$

- Current Contents ${ }^{\circledR}$ / Physical, Chemical, and Earth Sciences ${ }^{\circledR}$

- Scopus ${ }^{\circledR}$

Geodiversitas est distribué en version électronique par / Geodiversitas is distributed electronically by:

- BioOne ${ }^{\circledR}$ (http://www.bioone.org)

Les articles ainsi que les nouveautés nomenclaturales publiés dans Geodiversitas sont référencés par / Articles and nomenclatural novelties published in Geodiversitas are referenced by:

- ZooBank ${ }^{\circledR}$ (http://zoobank.org)

Geodiversitas est une revue en flux continu publiée par les Publications scientifiques du Muséum, Paris Geodiversitas is a fast track journal published by the Museum Science Press, Paris

Les Publications scientifiques du Muséum publient aussi / The Museum Science Press also publish:

Adansonia, Zoosystema, Anthropozoologica, European Journal of Taxonomy, Naturae, Cryptogamie sous-sections Algologie, Bryologie, Mycologie

Diffusion - Publications scientifiques Muséum national d'Histoire naturelle

CP $41-57$ rue Cuvier F-75231 Paris cedex 05 (France)

Tél. : 33 (0)1407948 05 / Fax: 33 (0)14079 3840

diff.pub@mnhn.fr / http://sciencepress.mnhn.fr

(C) Publications scientifiques du Muséum national d'Histoire naturelle, Paris, 2019

ISSN (imprimé / print): 1280-9659/ ISSN (électronique / electronic): 1638-9395 


\title{
L'apport du registre paléogène d'Amazonie sur la diversification initiale des Caviomorpha (Hystricognathi, Rodentia) : implications phylogénétiques, macroévolutives et paléobiogéographiques
}

\author{
Myriam BOIVIN \\ Laboratoire de Planétologie et Géodynamique (LPG), UMR 6112, CNRS, \\ Université de Nantes, 2 Chemin de la Houssinière, F-44300 Nantes cedex 3 (France) \\ et Institut des Sciences de l'Évolution de Montpellier, \\ UMR 5554, CNRS, IRD, EPHE, Université de Montpellier, \\ Place Eugène Bataillon, F-34095 Montpellier cedex 05 (France) \\ myriam.boivin@univ-nantes.fr \\ myriam.boivin@umontpellier.fr \\ Laurent MARIVAUX \\ Pierre-Olivier ANTOINE \\ Institut des Sciences de l'Évolution de Montpellier, \\ UMR 5554, CNRS, IRD, EPHE, Université de Montpellier, \\ Place Eugène Bataillon, F-34095 Montpellier cedex 05 (France) \\ laurent.marivaux@umontpellier.fr \\ pierre-olivier.antoine@umontpellier.fr
}

Soumis le 13 avril 2018 | accepté le 8 novembre 2018 | publié le 28 février 2019

urn:Isid:zoobank.org:pub:9DCB8512-42BB-4010-BCEC-DF38479499D5

Boivin M., Marivaux L. \& Antoine P.-O. 2019. - L'apport du registre paléogène d'Amazonie sur la diversification initiale des Caviomorpha (Hystricognathi, Rodentia): implications phylogénétiques, macroévolutives et paléobiogéographiques. Geodiversitas 41 (4): 143-245. https://doi.org/10.5252/geodiversitas2019v41a4. http://geodiversitas.com/41/4

\section{RÉSUMÉ}

Les rongeurs caviomorphes constituent l'un des groupes de mammifères placentaires les plus diversifiés d'Amérique du Sud. Malgré la grande diversité taxonomique actuelle et un riche registre néogène, les premières phases de l'histoire évolutive du groupe n'étaient, il y a peu, documentées que par quelques localités, majoritairement situées aux moyennes et hautes latitudes du continent sud-américain. Des recherches paléontologiques récentes menées en Amazonie péruvienne (régions de Contamana et Tarapoto) ont permis la découverte d'une vingtaine de localités éocènes et oligocènes, livrant de nombreux restes dentaires de caviomorphes. Les études systématiques effectuées sur ce matériel ont conduit à la description de nombreuses nouvelles espèces. Une analyse cladistique de grande ampleur (107 taxons terminaux; 513 caractères morphologiques), incluant ces nouvelles espèces amazoniennes, a été réalisée ici. Pour la première fois, les quatre super-familles actuelles (i.e., Cavioidea, Erethizontoidea, Chinchilloidea, et Octodontoidea) y sont largement représentées, notamment par l'ensemble des familles actuelles, elles-mêmes documentées par plusieurs représentants fossiles et/ou actuels. Cette analyse a permis la reconnaissance de caviomorphes basaux, n'appartenant à aucune de ces super-familles. Les relations de parenté étroites entre Erethizontoidea et Cavioidea d'une part, et Octodontoidea et Chinchilloidea d'autre part, comme proposé dans des phylogénies moléculaires récentes, apparaissent soutenues par les caractères morpho-anatomiques. Ce constat mène à la défi- 
MOTS CLÉS

Amérique du Sud, Pérou,

Cavioidea

Chinchilloidea,

Erethizontoidea,

Octodontoidea

rongeurs caviomorphes, morphologie,

analyses cladistiques,

macroévolution,

taxons nouveaux,

genre nouveau,

statut nouveau,

combinaison nouvelle.

KEY WORDS

South America,

Peru,

Cavioidea

Chinchilloidea

Erethizontoidea,

Octodontoidea

caviomorph rodents,

morphology,

cladistic analyses,

macroevolution,

new taxa,

new genus,

new status,

new combination. nition formelle des clades Erethicavioi Boivin, n. taxon (Erethizontoidea + Cavioidea + taxons éteints apparentés) et Octochinchilloi Boivin, n. taxon (Octodontoidea + Chinchilloidea + taxons éteints apparentés). Une classification synthétique des Caviomorpha est proposée. Les résultats révèlent également l'existence de trois phases de diversifications majeures au cours du Paléogène et du Miocène inférieur (radiation initiale des caviomorphes; émergence des super-familles actuelles; diversification des super-familles avec l'émergence de familles/sous-familles actuelles). Ces phases semblent coïncider avec des évènements climatiques globaux et des périodes de surrection andine intense. À la lumière de ces nouvelles données paléontologiques et des résultats phylogénétiques présentés ici, il semblerait que les régions de basses latitudes du continent sud-américain aient été le siège de la première phase de diversification des caviomorphes. L'origine géographique des super-familles reste quelque peu ambiguë, à l'exception toutefois des chinchilloïdes qui semblent avoir émergé dans les régions de basses latitudes.

\section{ABSTRACT}

New insight from the Paleogene record of Amazonia into the early diversification of Caviomorpha (Hystricognathi, Rodentia): phylogenetic, macroevolutionary, and paleobiogeographic implications.

Caviomorph rodents represent one of the most successful groups of placental mammals from South America. Despite their modern and Neogene high taxonomic diversity, the early evolutionary history of caviomorphs has long remained obscure. Until recently, the majority of Paleogene caviomorph assemblages were limited to South American localities at middle and high latitudes, whereas only a few were known at lower latitudes. Recent field expeditions in Peruvian Amazonia (Contamana and Tarapoto areas) have led to the discovery of about twenty Eocene and Oligocene localities yielding hundreds of fossils of caviomorphs (mainly isolated teeth). The systematic studies of these fossils have revealed the presence of numerous new species. A large-scale cladistic analysis (107 terminal taxa; 513 morphological characters), including these new Amazonian species, was undertaken here. For the first time, the four modern superfamilies (i.e., Erethizontoidea, Cavioidea, Octodontoidea, and Chinchilloidea) and all extant families were considered and included through modern and fossil terminal taxa. As a result, several stem Caviomorpha were recognized outside all four superfamilies. Close relationships between Erethizontoidea and Cavioidea on one hand and Octodontoidea and Chinchilloidea on the other hand, as hypothesized in most recent molecular phylogenies, are supported morphologically, thus leading to the formal definition of the clades Erethicavioi Boivin, n. taxon (Erethizontoidea + Cavioidea + extinct related taxa) and Octochinchilloi Boivin, n. taxon (Octodontoidea + Chinchilloidea + extinct related taxa). A synthetic classification of Caviomorpha is proposed. Three main diversification phases would have occurred during the Paleogene and early Miocene (early radiation of caviomorphs; emergence of modern superfamilies; diversification of superfamilies with emergence of some modern families/subfamilies). These three phases seemingly coincide with global climatic events and intense Andean uplift phases. Based on these recent fossil discoveries and on the phylogenetic results presented here, low latitudes of South America are viewed as the first diversity hotspot of early caviomorphs. Geographic origin of modern superfamilies remains somewhat ambiguous, except for chinchilloids which would have emerged in low-latitude regions.

\section{EXTENDED ABSTRACT}

Caviomorph rodents constitute one of the most successful groups of placental mammals from South America. They are represented nowadays by four superfamilies: Cavioidea (guinea pigs and their allies), Chinchilloidea (chinchillas and their allies), Octodontoidea (spiny rats and their allies) and Erethizontoidea (New World porcupines). Despite their modern and Neogene high taxonomic diversity, the early evolutionary history of caviomorphs has long remained obscure. Until recently, the majority of Paleogene caviomorph assemblages were limited to South American localities at middle and high latitudes, whereas only a few were known at lower latitudes. In these low-latitude regions, the Paleogene record of caviomorphs was essentially known through a single locality: Santa Rosa (Peruvian Amazonia; late ?Eocene/early Oligocene). Recent field expeditions in Peruvian Amazonia (Contamana and Tarapoto areas) have led to the discovery of about twenty Eocene and Oligocene localities, yielding hundreds of fossils of caviomorphs (mainly isolated teeth) in a well-constrained stratigraphic framework. The systematic studies of these fossils have revealed the presence of numerous new species. The late middle Eocene localities from Contamana (c. $41 \mathrm{Ma}$ ) have so far yielded the earliest representatives of caviomorphs including mostly taxa that harbor very primitive dental features. Besides, the latter localities and the younger Santa Rosa assemblage would document the earliest representatives of the octodontoids, cavioids and erethizontoids. The Oligocene caviomorph assemblages from Tarapoto/ Shapaja (early Oligocene) and Contamana (late Oligocene) show a higher morpho-dental disparity linked to the presence of at least three of the four superfamilies (i.e., octodontoids, chinchilloids, and erethizontoids). Amazonia (i.e., Proto-Amazonia at those times) has therefore played a substantial role 
in the early radiation(s) of caviomorphs. The current work aims to undertake a large-scale cladistic analysis with a hundred of terminal taxa, notably Paleogene and including several Amazonian species from Contamana and Tarapoto areas. For the first time, all four modern superfamilies and all extant families were considered and included through modern and fossil terminal taxa. A matrix was assembled in observing/describing 513 morphological characters ( 432 dental and 81 cranio-mandibular) through a comprehensive taxonomic sampling (116 terminal taxa). Many of these characters were based on those from the matrix realised by L. Marivaux for the phylogenetic analysis published in Antoine et al. (2012). Nevertheless, additional characters based on new observations, associated with the inclusion of characters from other cladistic analyses allowed to modify, subtantially complement, and then considerably expand the morphological dataset from the initial matrix. The ingroup was composed of 96 caviomorph taxa (35 Paleogene, 36 Miocene, one Pliocene, and 24 extant). We selected as outgroups three taxa among which one basal glire and two basal rodents, and as branching-groups, 17 taxa among which one ischyromyoid and 16 (a priori) non-caviomorph Ctenohystrica (15 extinct and one extant). Terminals were mainly scored at the specific level. Several cladistic analyses with a different number of taxa were performed (AG0-AG9) with PAUP* v.4.0 beta 10 Win. The analyses AG0 and AG1 gathered the highest number of taxa (107 out of 116). AG0 was totally unconstrained, whereas AG1 was constrained by a molecular scaffold in order to recover the caviomorph clades that are strongly supported by genomic sequences. In these two general analyses, nine taxa (the three species of Gaudeamus, Loretomys, Plesiosteiromys, Cephalomys arcidens, Soriamys, Litodontomys, and "Asteromys» bolivianus) were excluded. Indeed, their joint inclusion in AG0 and AG1 generated too many topologic irresolutions. In fact, these taxa have proven to have an ambiguous superfamilial status (in displaying convergent characters with different representatives of distinct superfamilies), or are usually poorly documented. They were treated subsequently in incorporating them one by one in the dataset and global parameters of the AG1 (analyses AG2-AG9).

The results of the AG1 have revealed that several early caviomorphs occupied a phylogenetic position outside the four main superfamilies, and as such were considered as stem Caviomorpha. The latter originate from Eocene localities of Contamana (eight species: Cachiyacuy kummeli, Cachiyacuy contamanensis, Canaanimys maquiensis, Pozomys ucayaliensis, Tarapotomys mayoensis, Tarapotomys subandinus, Shapajamys labocensis, and Eobranisamys javierpradoi) and from Santa Rosa (two species: Eoespina woodi = Eosachacui lavocati and Eobranisamys romeropittmanae). Following the phylogenetic results of the AG1 (incorporating a molecular scaffold), we decided here to name the clade including the Erethizontoidea + the Cavioidea and its extinct related taxa (?Paleosteiromys): Erethicavioi Boivin, n. taxon. Similarly, the clade uniting the Octodontoidea + the Chinchilloidea and its extinct related taxa (Eosallamys and Mayomys) was named here: Octochinchilloi Boivin, n. taxon. Within these high level clades, no stem Cavioidea or stem Erethizontoidea were recognized (with the possible exception of Paleosteiromys), whereas many basal taxa were regognized as stem Octodontoidea and stem Chinchilloidea. Following our results, several previously alleged dasyproctids would be stem Caviomorpha (Eobranisamys) or stem Chinchilloidea (Eoincamys and Microscleromys) instead. The two species of Chambiramys (C. sylvaticus and C. shipiborum) and Scleromys praecursor from the Late Oligocene localities of Contamana would be also stem Chinchilloidea. Scleromys praecursor being phylogenetically remote from other species of Scleromys (including the type species $S$. angustus), we reassigned this species to the new genus Maquiamys n. gen., under the binomen Maquiamys praecursor n. comb. The results of supplementary analyses supported phylogenetic affinities: i) within chinchilloid caviomorphs for all three species of the African Gaudeamys (AG2); ii) among stem Caviomorpha for Plesiosteiromys (AG8); and iii) among stem Octodontoidea for Loretomys (AG9). According the results of AG3-AG7, Litodontomys and "Asteromys" bolivianus would be included within cepalomyids, contrary to Soriamys. The position of Asteromys punctus is ambiguous (either as a Cephalomyidae or a Cavioidea). The superfamilail status of cephalomyids was not recovered here. A synthetic classification of Caviomorpha is proposed. Transposed into a chronostratigraphical context, the strict consensus tree of the eight equally most parcimonious trees generated by the AG1 revealed the existence of several ghost-lineages. They are particularly abundant for post-Miocene caviomorphs, and therefore likely artefactual, being related to the taxonomic sampling of the analysis (post-Miocene fossil caviomorphs are underrepresented with respect to both Eocene-Miocene and recent taxa). Other ghost-lineages are implied during two key periods of the evolutionary history of caviomorphs: around the divergence of crown Caviomorpha (middle Eocene-late Eocene), the emergence of the superfamilies (late Eocene-early Oligocene), and within each superfamily (notably regarding their stem taxa; Oligocene and ranging up to the early Miocene for the chinchilloids and erethizontoids). Many dental characters, evolutionary trends or character associations diagnosing each superfamily are detailed and listed. In this context, we then also discuss here the evolutionary history of the dental morphology of caviomorphs during the Paleogene. 
From a macroevolutionary perspective, our phylogenetic results point out the existence of three main diversification phases: i) the early radiation of caviomorphs at the end of the Middle Eocene; ii) the emergence of modern superfamilies around the Eocene-Oligocene transition; and iii) their diversification with the emergence of several modern families/subfamilies around the Oligocene-Miocene transition. Interestingly, these three phases seemingly would coincide with well-identified global climatic events and intense Andean uplift phases. Indeed, the end of the middle Eocene records a global warming (the Mid-Eocene Climatic Optimum [MECO]) and the main period of the Andean Orogeny of Peru (Inca phase). The Eocene-Oligocene transition is marked by a strong global cooling (the Eocene-Oligocene Boundary cooling $[\mathrm{EOBc}]$ ), the end of the Inca phase and the beginning of a significant shortening in Eastern Cordillera Central Andes. Finally, at the Oligocene-Miocene transition, a global cooling (the Oligocene-Miocene Boundary cooling [OMBc]) is recorded, and this time window is also the beginning of the Quechua orogenetic phase. These different global climatic changes associated with distinct pulses of the Andean uplifts could have driven the early diversifications of caviomorph rodents. It is clear that the evolutionary history of the group was complex and might have been influenced by a combinaison of biotic and abiotic factors, acting at different spatio-temporal scales.

Based on these recent fossil discoveries and on phylogenetic results presented here, low latitudes of South America are thus viewed as the first diversity hotspot of early caviomorphs. The geographic origin of modern superfamilies remains somewhat ambiguous, except for chinchilloids, which would have emerged in regions of low-latitudes. After a pioneering transatlantic dispersal from Africa, the original differentiation of crown Caviomorpha in South America has probably occurred in lowland tropical rainforest at low latitudes during the late middle and late Eocene. From low-latitudes of the Neotropics, caviomorphs would have subsequently dispersed toward middle- and high-latitudes. However, the chronology, tempo and modalities of these dispersals, as well as the pathways by which these dispersals took place remain difficult to address. Our results would favor to the hypothesis of geographic areas expanding independently for each superfamily around the Eocene-Oligocene transition. Perhaps due to a richer fossil record than at low- and mid-latitudes, the Southern cone of South America clearly was a distinctive center of taxonomic diversification for the four modern superfamilies. The different geographic origins of the superfamilies as suggested by AG1, notably between erethizontoids and cavioids, and between chinchilloids and octodontoids, suggest an incomplete EoceneOligocene record of the group for both low- and high-latitudes. Despite their central position, the minor role played by mid-latitude regions may also suggest the existence of extensive gaps in the fossil record of this geographical area. Lastly, several dispersal events would have occurred between these regions during the Oligocene epoch. They would at least have concerned different octodontoids, erethizontoids and chinchilloids, with a noticeable expansion of the corresponding distribution areas.

\section{INTRODUCTION}

Les rongeurs caviomorphes constituent l'un des groupes de mammiferes placentaires les plus diversifiés d'Amérique du Sud. Ils sont étroitement apparentés aux phiomorphes (Phiomorpha sensu lato ou hystricognathes de l'Ancien Monde; e.g. Lavocat 1969; Huchon \& Douzery 2001; Marivaux et al. 2002, 2004; Antoine et al. 2012; Fabre et al. 2012) au sein de l'infra-ordre Hystricognathi Tullberg, 1899. Le groupe représente un modèle biologique d'intérêt avec ses c. 244 espèces actuelles (soit c. 11\% de la diversité spécifique totale des rongeurs; e.g. Upham \& Patterson 2015) regroupées en quatre super-familles: Cavioidea (cochon d'Inde et formes apparentées), Chinchilloidea (chinchilla, viscaches et pacaranas), Octodontoidea (rats épineux et formes apparentées) et Erethizontoidea (porcs-épics du Nouveau Monde). Ils présentent une énorme diversité morphologique et sont caractérisés par un large spectre d'adaptations écomorphologiques illustrant plusieurs modes de vie (terrestre, fouisseur, arboricole et semi-aquatique; e.g. Patton et al. 2015).
Malgré leur grande diversité actuelle et un riche registre néogène, les premières phases de l'histoire évolutive des caviomorphes n'étaient, il y a peu, documentées que par quelques localités, majoritairement situées aux moyennes et hautes latitudes du continent sud-américain. L'Amazonie, située dans les Néotropiques de basses latitudes, est l'une des zones géographiques concentrant de nos jours le maximum de la richesse spécifique des caviomorphes (Upham \& Patterson 2015). Toutefois, la paléobiodiversité de cette zone géographique est longtemps restée sous-documentée en raison de conditions particulières de préservation des restes fossiles, du couvert végétal dense, et des conditions difficiles de travail en milieu tropical. Jusqu'à récemment, le registre paléogène des caviomorphes y était essentiellement connu par une seule localité: Santa Rosa (Amazonie péruvienne; ?Éocène supérieur/Oligocène inférieur; Frailey \& Campbell 2004). Des recherches paléontologiques récentes menées en Amazonie péruvienne (régions de Contamana et de Tarapoto) ont, depuis, permis la découverte de 18 
localités éocènes et oligocènes, livrant de nombreux restes dentaires de caviomorphes (Antoine et al. 2012, 2016, 2017 ; Boivin et al. 2017a, b, 2018a, b). Ces nouvelles localités d'Amazonie péruvienne documentent trois étages mammaliens sud-américains (SALMAs: South American Land Mammal Ages) du Paléogène (Antoine et al. 2016, 2017 ; Boivin et al. 2018a) : Barrancien (fin Éocène moyen; Contamana, Formation Pozo [membre inférieur]), Tinguiriricien (Oligocène inférieur; Tarapoto, Formation Pozo [membre supérieur]) et Déséadien (Oligocène supérieur; Contamana, Formation Chambira). Les études systématiques précédentes effectuées sur ce matériel ont conduit à la description de 52 taxons distincts, dont 17 nouvelles espèces formellement nommées (Antoine et al. 2012; Boivin et al. 2017a, b, 2018a). Les localités de Contamana datées de la fin de l'Éocène moyen livrent en particulier les plus anciens caviomorphes connus à ce jour (c. $41 \mathrm{Ma}$; Antoine et al. 2016; Boivin et al. 2017a). Certains de ces rongeurs sont caractérisés par des traits dentaires plésiomorphes, rappelant les formes hystricognathes de l'Ancien Monde qui leur sont subcontemporaines (Antoine et al. 2012; Boivin et al. 2017a). En outre, ces localités de Contamana et celle de Santa Rosa documenteraient les plus anciens octodontoïdes, cavioïdes et éréthizontoïdes (Frailey \& Campbell 2004; Antoine et al. 2012; Boivin et al. 2017a). Les assemblages de caviomorphes de Tarapoto (Shapaja; Boivin et al. 2018a) et de l'Oligocène supérieur de Contamana (Boivin et al. 2017b) montrent quant à elles une riche diversité alpha-taxonomique et une disparité morphologique importante associées à la présence d'au moins trois des quatre superfamilles (i.e., octodontoïdes, chinchilloïdes et éréthizontoïdes). Ainsi, la zone géographique de l'actuelle Amazonie aurait abrité au Paléogène des faunes variées de caviomorphes, et ce dès les premières phases de leur histoire évolutive. Cette région de basses latitudes a manifestement joué un rôle majeur dans la/ les radiation(s) initiale(s) du groupe, juste après son arrivée sur le continent sud-américain à partir de l'Afrique (avant c. $41 \mathrm{Ma}$; Antoine et al. 2012).

Bien que les relations phylogénétiques des caviomorphes modernes soient aujourd'hui mieux établies (e.g. Voss et al. 2013; Upham \& Patterson 2015; Fabre et al. 2017), les positions phylogénétiques de nombreux représentants éteints du groupe, notamment paléogènes, restent cependant incertaines ou ambiguës. Plusieurs facteurs peuvent expliquer ce manque de résolution: un manque de données paléontologiques rendant difficile la documentation des taxons et la compréhension de l'évolution morphologique de certains groupes (e.g. Cavioidea; Vucetich et al. 2015a), des homoplasies dentaires pouvant être fréquentes selon les caractères considérés (e.g. hypsodontie, taeniodontie, etc.), une définition des super-familles souvent ancienne et s'appuyant rarement sur des synapomorphies non-ambiguës, l'existence de groupes «fourre-tout» (e.g. Dasyproctidae) et celle de lignées fantômes manifestes, notamment aux basses latitudes de l'Amérique du Sud. Récemment, des analyses phylogénétiques, notamment de parcimonie, ont permis la révision taxonomique de plusieurs groupes de caviomorphes. À l'échelle suprafamiliale, les études se sont concentrées principalement sur les Cavioidea (Pérez 2010a, b ; Pérez \& Vucetich 2011, 2012a, b ; Pérez \& Pol 2012; Pérez et al. 2012) et les Octodontoidea (Carvalho \& Salles 2004; Candela \& Rasia 2012; Arnal et al. 2014, 2017; Arnal \& Vucetich 2015a; Candela 2016; Verzi et al. 2014, 2016) et, dans une bien moindre mesure, sur les Chinchilloidea (Kramarz et al. 2013; Kerber et al. 2018) et Erethizontoidea (Candela 2000, 2001, 2004).

Ce travail consiste en la réalisation d'une analyse cladistique de grande ampleur incluant plus d'une centaine de taxons terminaux, notamment paléogènes (dont la majorité des espèces de Contamana et de Tarapoto récemment décrites; Boivin et al. 2017a, b, 2018a). Pour la première fois, les quatre superfamilles y sont représentées par un grand nombre de familles (toutes les familles actuelles sont considérées), elles-mêmes documentées par plusieurs représentants fossiles et/ou actuels. Cette analyse a pour but d'établir un cadre phylogénétique de la radiation initiale des caviomorphes et de formaliser la position de ces nouvelles espèces découvertes en Amazonie péruvienne. En outre, les résultats nous permettront d'évaluer le rôle de ces espèces fossiles dans la compréhension des relations inter- et intrafamiliales des caviomorphes. Replacés dans un contexte temporel, ces résultats phylogénétiques offriront un cadre robuste et réfutable, afin de mieux appréhender les premiers temps de l'histoire évolutive des caviomorphes, et en particulier l'émergence des familles et des super-familles modernes.

\section{MATÉRIEL ET MÉTHODES}

\section{CARACTÈRES}

\section{Choix des caractères}

Une matrice composée de 513 caractères, essentiellement dentaires (432) mais aussi crâniens/mandibulaires (81), a été assemblée (Annexes 1 et 2) à l'aide du logiciel Nexus Data Editor (NDE; Page 2001). Les 432 caractères dentaires se divisent en trois sous-partitions:

- les 20 premiers caractères portent sur la morphologie globale des dents (supérieures et inférieures, les incisives inclues) et la microstructure de l'émail des incisives;

- les 231 suivants portent sur la morphologie occlusale des dents supérieures (P3, dP4, P4, M1-3);

- et les 181 derniers portent sur la morphologie occlusale des dents inférieures (dp4, p4, m1-3).

Nombre de ces caractères sont issus de ceux proposés par L. Marivaux à l'occasion de l'analyse phylogénétique publiée par Antoine et al. (2012). Toutefois, des observations nouvelles et la prise en compte d'autres analyses cladistiques (Carvalho \& Salles 2004; Candela \& Rasia 2012; Pérez et al. 2012; Kramarz et al. 2013; Arnal \& Vucetich 2015a; Verzi et al. 2016) ont permis de modifier, d'agrémenter substantiellement, et ainsi d'élargir considérablement le corpus de données morphologiques de la matrice initiale, publiée en données supplémentaires par Antoine et al. (2012). Les observations nouvelles ont été effectuées directement sur le matériel (originaux ou moulages) ou à partir de photographies et de 
A

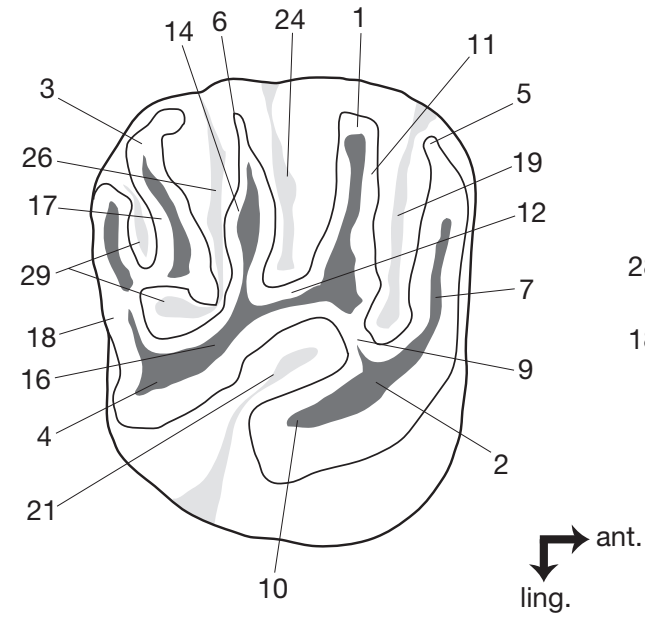

D

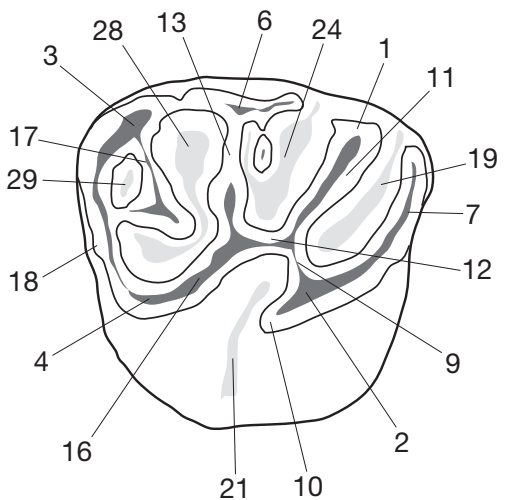

B

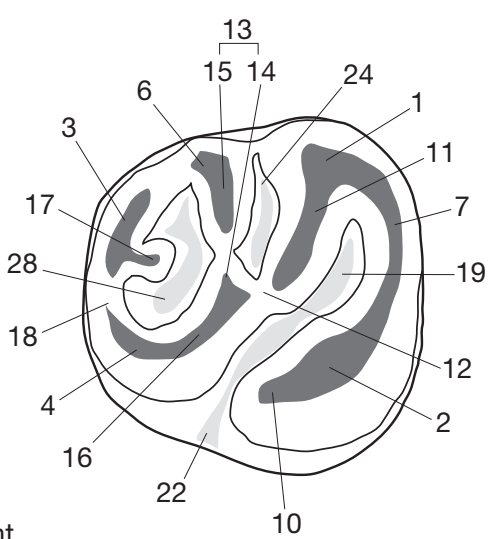

$\mathrm{E}$

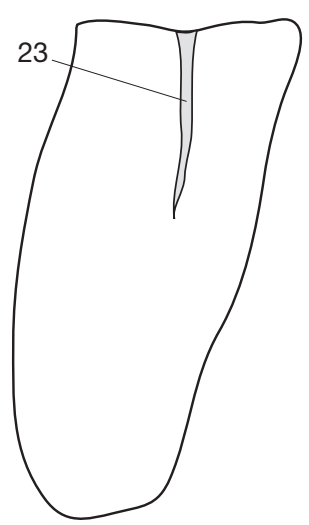

C
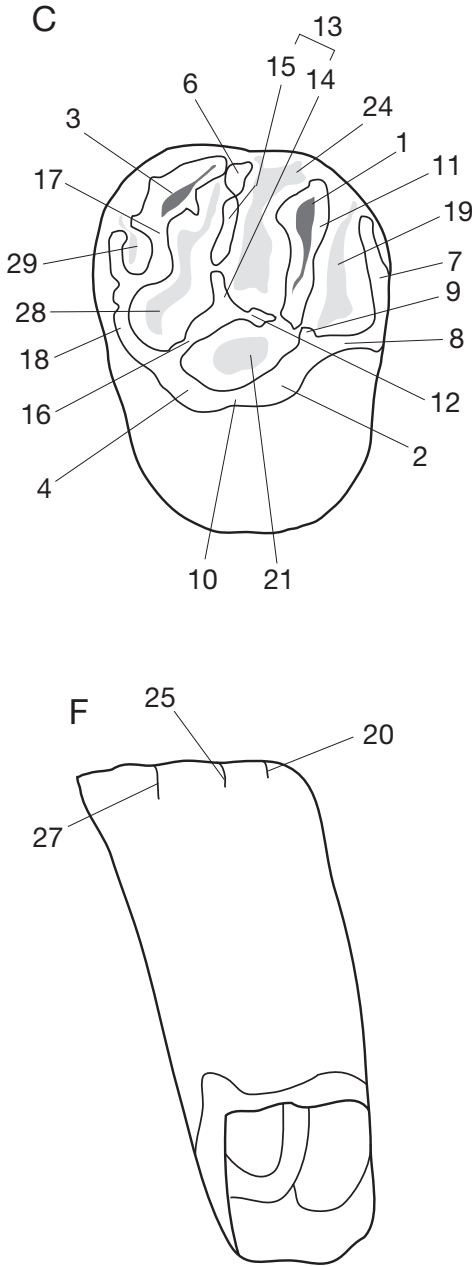

FIG. 1. - Nomenclature des dents supérieures utilisée dans le présent travail, basée sur celle proposée dans Boivin et al. (2017b): A, B, molaire supérieure, vue occlusale; A, molaire pentalophodonte; $\mathbf{B}$, molaire tétralophodonte; $\mathbf{C}$, P4, vue occlusale; $\mathbf{D}$, dP4, vue occlusale; $\mathbf{E}, \mathbf{F}$, dent supérieure; E, vue linguale; $\mathbf{F}$, vue labiale. Abréviations: ant, antérieur; ling, lingual; 1, paracône; 2, protocône; 3, métacône; 4, hypocône; 5, parastyle; 6, mésostyle; 7 , antérolophe; 8 , bras antérieur du protocône; 9 , protolophe lingual (= bras postérieur du protocône); 10, excroissance postérieure du protocône; 11, protolophe (= protolophe labial); 12, mure; 13, troisième crête transverse (= crête transverse centrale); 14, mésolophule; 15, mésolophe; 16, bras antérieur de l'hypocône; 17, métalophe; 18, postérolophe; 19, paraflexus; 20, parastria; 21, hypoflexus/hypofossette; 22 , confluence du paraflexus avec l'hypoflexus; 23, hypostria; 24 , mésoflexus/ mésofossette mésial; 25, mésostria; 26, mésoflexus/mésofossette distal; 27 , métastria; 28 , confluence du mésoflexus distal avec le métaflexus; 29 , métaflexus.

figurations issues de la littérature (Annexe 3). L'observation approfondie du matériel provenant des régions de Contamana et de Tarapoto a notamment permis de proposer de nouvelles hypothèses d'homologies dentaires chez les caviomorphes. Ces hypothèses ont servi de base à l'élaboration de certains caractères. Ces hypothèses sont synthétisées et plus amplement discutées dans la thèse de Boivin (2017) et dans Boivin \& Marivaux (2018).

Il n'a pas été possible d'intégrer des caractères postcrâniens à cette analyse: le squelette axial et appendiculaire des caviomorphes a été peu étudié chez les formes actuelles et le registre fossile est à la fois extrêmement lacunaire et largement méconnu (e.g. Wood 1949; Sánchez-Villagra et al. 2003; Horovitz et al. 2006; Candela \& Picasso 2008; Candela et al. 2012; Olivares \& Verzi 2015). De fait, ces caractères auraient été très majoritairement codés manquants et la distribution de leurs états aurait été ambiguë, minimisant ainsi considérablement leur portée.
La constitution et/ou la modification des caractères et de leurs états ont obéi à plusieurs critères:

- les caractères choisis varient entre espèces et taxons de rang taxonomique supérieur;

- de nombreux caractères, bien que variables au sein d'une même espèce (i.e., polymorphiques sensu Wiens 1995 et Wiens \& Servedio 1997), ont été sélectionnés car ils témoignent, cependant, de tendances différentes entre certaines espèces. Certains auteurs ont mis en évidence que ce type de caractères pouvait être porteur de signal phylogénétique (e.g. Wiens 1995; Wiens \& Servedio 1997); - les caractères dentaires ont été codés si possible sur des spécimens frais ou peu usés, sauf mention contraire, afin de limiter l'effet de l'usure pouvant être source de polymorphisme; - les caractères ont porté sur des structures considérées a priori comme homologues chez les taxons terminaux (i.e., homologie primaire);

- chaque caractère correspond à un attribut d'une structure (présence/absence, forme, développement, etc.), celui-ci variant 
A

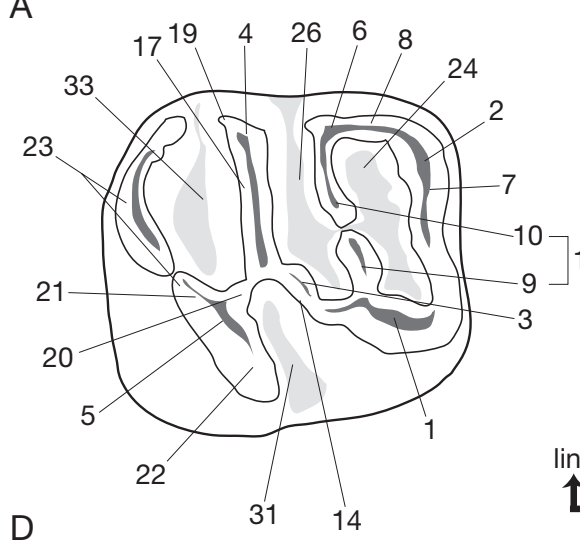

B

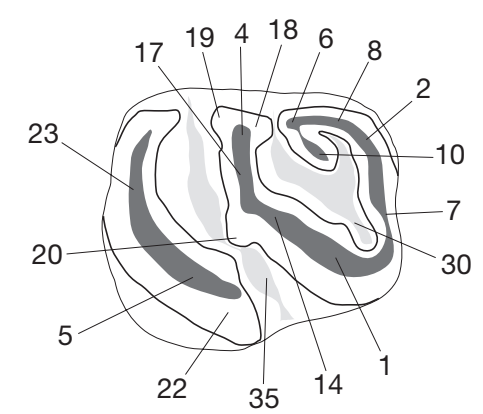

$\stackrel{\uparrow}{\longrightarrow}$ ant.

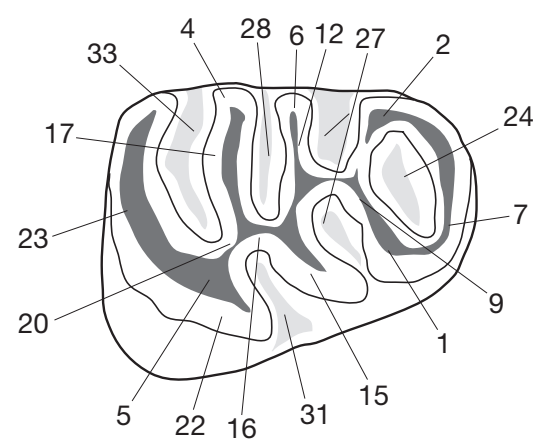

$\mathrm{E}$

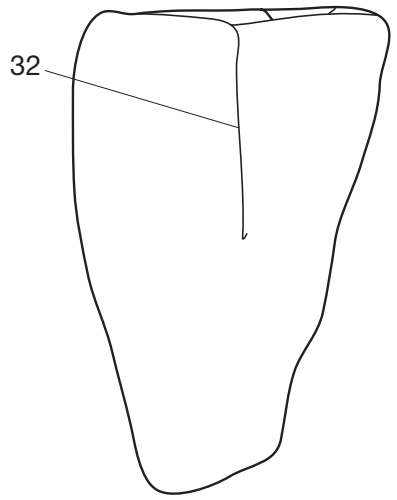

C
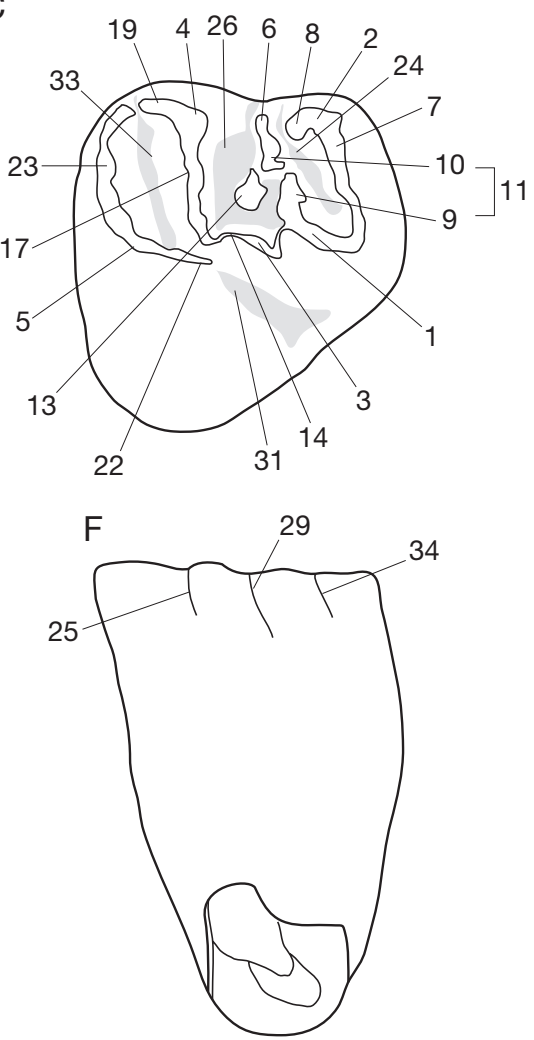

FIG. 2. - Nomenclature des dents inférieures utilisée dans le présent travail, basée sur celle proposée dans Boivin et al. (2017b): A, B, molaire inférieure, vue occlusale; A, molaire tétralophodonte; B, molaire trilophodonte/tétralophodonte; C, P4, vue occlusale; D, dP4, vue occlusale; E, F, dent supérieure; $\mathbf{E}$, vue labiale; $\mathbf{F}$, vue linguale. Abréviations: ant, antérieur; ling, lingual; $\mathbf{1}$, protoconide; $\mathbf{2}$, métaconide; $\mathbf{3}$, mésoconide; $\mathbf{4}$, entoconide; $\mathbf{5}$, hypoconide; $\mathbf{6}$ mésostylide; $\mathbf{7}$, métalophulide I; $\mathbf{8}$, bras postérieur du métaconide; $\mathbf{9}$, bras postérieur du protoconide; 10 , néomésolophide; $\mathbf{1 1}$, deuxième cristide transverse; 12, mésolophide; 13, reste de mésolophide ?; 14, ectolophide; 15, ectolophide mésial; 16, ectolophid distal; 17, hypolophide; 18, bras antérieur de l'entoconide; 19, bras postérieur de l'entoconide; 20, bras antérieur de l'hypoconide; 21, bras postérieur de l'hypoconide; 22 , excroissance antérieure de I'hypoconide; 23, postérolophide; 24 , antéroflexide/antérofossettide; 25, antérostriide; 26 , mésoflexide; 27 , mésoflexide mésial; 28 , mésoflexide distal; 29, mésostriide; 30, confluence de l'antéroflexide avec le mésoflexide; $\mathbf{3 1}$, hypoflexide; $\mathbf{3 2}$, hypostriide; 33, métaflexide; 34, métastriide; 35, confluence de l'hypoflexide avec le métaflexide.

a priori de façon indépendante par rapport aux autres attributs de cette même structure (codage 3 sensu Pleijel 1995);

- les caractères sont binaires ou à états multiples (ordonnés ou non ordonnés, voir section "Traitement des caractères"); - la décomposition des caractères en "états» est le reflet de toute la gamme morphologique observée pour les structures considérées sur l'ensemble des taxons étudiés;

- la taille globale n'a pas été prise en compte et les caractères quantitatifs ont été traduits par des rapports (e.g. indice d'hypsodontie, largeur d'une dent par rapport à sa longueur, etc.).

\section{Nomenclatures utilisées}

La nomenclature dentaire principale utilisée dans ce volume (Figs 1 ; 2) suit celle proposée par M. Boivin et L. Marivaux dans Boivin et al. (2017b). Cette nomenclature est parfois difficile d'emploi, particulièrement chez certains caviomorphes actuels (et les formes fossiles qui leur sont directement apparentées) arborant une morphologie dentaire très différente de celle des plus anciens représentants de ce groupe, caractérisés par des dents brachyodontes, buno-lophodontes et des molaires supérieures et inférieures pentalophodontes/ tétralophodontes (Antoine et al. 2012). C'est le cas, par exemple des Hydrochoerinae, pour lesquels une nomenclature particulière (Vucetich et al. 2005, 2013) a été suivie pour les caractères 250, 251, 299-301 et 428-432.

Les caractères 433-513 sont fondés sur les nomenclatures crâniennes et mandibulaires de Hill (1935), Schaub (1958), Wahlert (1985), Novacek (1993), Emmons (2005), Wible et al. (2005), Pérez (2010a), Olivares \& Verzi (2015) et Verzi et al. (2016).

\section{Traitement des caractères}

Certains caractères portant sur une même structure ou sur des structures associées ou en interaction (e.g. dents supérieures et inférieures en occlusion durant la mastication) peuvent être interdépendants. Afin de limiter la redondance de l'information morphologique due à leur interdépendance, seuls les caractères montrant des changements d'états exclusifs ont été conservés (i.e., les lignes de codage sont distinctes pour au moins un des taxons terminaux entre les deux caractères susceptibles a priori d'être interdépendants).

La plupart des caractères à états multiples correspondent à une suite additive d'états (transformation additive) et ont été considérés comme ordonnés mais non orientés (commande 
TABLEAU 1. - Loci dentaires et restes cranio-mandibulaires non codés (i.e., manquants ou dont l'état est trop fragmentaire/déformé pour permettre leur codage; cellules vertes) des taxons inclus dans les analyses cladistiques. Les cellules vertes claires (x) correspondent aux loci et aux restes cranio-mandibulaires non codés et les vertes foncées (+) aux p4/P4 non développées chez les taxons montrant une rétention des dp4/dP4. Les taxons pour lesquelles toutes ces structures sont documentées et dont l'état permet leur codage ne sont pas indiqués dans ce tableau.

\begin{tabular}{|c|c|c|c|c|c|c|c|c|c|c|}
\hline Taxons avec locus manquant & dP4 & P4 & M1-3 & dp4 & p4 & m1-3 & $\begin{array}{c}\text { Total loci } \\
\text { dentaire } \\
\text { manquant }\end{array}$ & 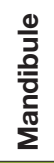 & 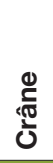 & $\begin{array}{c}\text { Total } \\
\text { structure } \\
\text { manquante }\end{array}$ \\
\hline Archetypomys erlianensis & - & $x$ & - & - & $x$ & - & $2 / 6$ & $\times$ & $\times$ & $4 / 8$ \\
\hline Tribosphenomys minutus & - & - & - & - & - & - & $0 / 6$ & - & $x$ & $1 / 8$ \\
\hline Cocomys lingchaensis & - & - & - & $x$ & - & - & $1 / 6$ & - & - & $1 / 8$ \\
\hline Reithroparamys delicatissimus & $x$ & - & - & $x$ & - & - & $2 / 6$ & - & - & $2 / 8$ \\
\hline Tamquammys dispinorum-wilsoni-tantillus & $x$ & - & - & $x$ & - & - & $2 / 6$ & - & $x$ & $3 / 8$ \\
\hline Chapattimys wilsoni & $x$ & - & - & $x$ & - & - & $2 / 6$ & $x$ & $x$ & $4 / 8$ \\
\hline Birbalomys sondaari-woodi & - & - & - & - & - & - & $0 / 6$ & - & $x$ & $1 / 8$ \\
\hline Ottomania proavita & - & - & - & - & - & - & $0 / 6$ & $x$ & $x$ & $2 / 8$ \\
\hline Baluchimys barryi & - & $x$ & - & - & $x$ & - & $2 / 6$ & $x$ & $x$ & $4 / 8$ \\
\hline Bugtimys zafarullahi & - & - & - & - & - & - & $0 / 6$ & - & $x$ & $1 / 8$ \\
\hline Hodsahibia azrae & - & $x$ & - & - & - & - & $1 / 6$ & $x$ & $x$ & $3 / 8$ \\
\hline «Waslamys» attiai & - & - & - & - & - & - & $0 / 6$ & - & $x$ & $1 / 8$ \\
\hline Phiomys hammudai & $x$ & - & - & - & - & - & $1 / 6$ & - & $x$ & $2 / 8$ \\
\hline Metaphiomys schaubi & - & + & - & - & + & - & $2 / 6$ & - & $x$ & $3 / 8$ \\
\hline Gaudeamus aslius & $x$ & - & - & - & - & - & $1 / 6$ & - & - & $1 / 8$ \\
\hline Gaudeamus hylaeus & $x$ & - & - & - & - & - & $1 / 6$ & - & $x$ & $2 / 8$ \\
\hline Gaudeamus aegyptius & $x$ & - & - & - & - & - & $1 / 6$ & - & $x$ & $2 / 8$ \\
\hline Paraphiomys pigotti & - & + & - & - & + & - & $2 / 6$ & - & - & $2 / 8$ \\
\hline Thryonomys swinderianus & - & + & - & - & + & - & $2 / 6$ & - & - & $2 / 8$ \\
\hline Cachiyacuy contamanensis & - & - & - & - & - & - & $0 / 6$ & $x$ & $x$ & $2 / 8$ \\
\hline Cachiyacuy kummeli & $x$ & - & - & - & - & - & $1 / 6$ & $x$ & $x$ & $3 / 8$ \\
\hline Canaanimys maquiensis & $x$ & $x$ & - & - & $x$ & - & $3 / 6$ & $x$ & $x$ & $5 / 8$ \\
\hline Protosteiromys medianus & $x$ & $x$ & - & $x$ & $x$ & - & $4 / 6$ & $x$ & $x$ & $6 / 8$ \\
\hline Eosteiromys homogenidens & $x$ & - & - & - & - & - & $1 / 6$ & - & - & $1 / 8$ \\
\hline Branisamyopsis australis-praesigmoides & $x$ & - & - & - & - & - & $1 / 6$ & - & $x$ & $2 / 8$ \\
\hline Neosteiromys pattoni & $x$ & - & - & $x$ & $x$ & - & $3 / 6$ & - & - & $3 / 8$ \\
\hline Erethizon dorsatum & $x$ & - & - & - & - & - & $1 / 6$ & - & - & $1 / 8$ \\
\hline Coendou prehensilis-insidiosus & - & - & - & $x$ & - & - & $1 / 6$ & - & - & $1 / 8$ \\
\hline Chaetomys subspinosus & - & - & - & $x$ & - & - & $1 / 6$ & - & - & $1 / 8$ \\
\hline Palaeosteiromys amazonensis & $x$ & $x$ & - & $x$ & $x$ & - & $4 / 6$ & $x$ & $x$ & $6 / 8$ \\
\hline Plesiosteiromys newelli & $x$ & $x$ & - & $x$ & $x$ & $x$ & $5 / 6$ & $x$ & $x$ & $7 / 8$ \\
\hline Eoincamys pascuali & $x$ & - & - & $x$ & - & - & $2 / 6$ & $x$ & $x$ & $4 / 8$ \\
\hline Eoincamys ameghinoi & $x$ & - & - & $x$ & - & - & $2 / 6$ & $x$ & $x$ & $4 / 8$ \\
\hline Eoincamys valverdei & $x$ & - & - & $x$ & $x$ & - & $3 / 6$ & $x$ & $x$ & $5 / 8$ \\
\hline Eoincamys parvus & $x$ & - & - & - & $x$ & - & $2 / 6$ & $x$ & $x$ & $4 / 8$ \\
\hline Eobranisamys romeropittmanae & $x$ & $x$ & - & $x$ & $x$ & - & $4 / 6$ & - & $x$ & $5 / 8$ \\
\hline Eobranisamys javierpradoi & - & - & - & - & - & - & $0 / 6$ & $x$ & $x$ & $2 / 8$ \\
\hline Litodontomys chubutomys & - & - & - & - & - & - & $4 / 6$ & $x$ & $x$ & $6 / 8$ \\
\hline Australoprocta fleaglei & - & - & - & - & - & - & $2 / 6$ & $x$ & $x$ & $4 / 8$ \\
\hline Cuniculus paca-taczanowskii & - & - & - & - & - & - & $2 / 6$ & - & - & $2 / 8$ \\
\hline Luantus propheticus & - & - & - & - & - & - & $0 / 6$ & - & $x$ & $1 / 8$ \\
\hline Asteromys punctus & $x$ & $x$ & $x$ & $x$ & - & - & $4 / 6$ & $x$ & $x$ & $6 / 8$ \\
\hline Neoreomys australis & $x$ & - & - & $x$ & - & - & $2 / 6$ & - & - & $2 / 8$ \\
\hline Dolichotis patagonum & $x$ & - & - & $x$ & - & - & $2 / 6$ & - & - & $2 / 8$ \\
\hline Hydrochoerus hydrochaeris & $x$ & - & - & $x$ & - & - & $2 / 6$ & - & - & $2 / 8$ \\
\hline Cavia aperea & $x$ & - & - & $x$ & - & - & $2 / 6$ & - & - & $2 / 8$ \\
\hline Galea spixii & $x$ & - & - & $x$ & - & - & $2 / 6$ & - & - & $2 / 8$ \\
\hline Cephalomys arcidens & $x$ & - & - & - & - & - & $1 / 6$ & - & - & $1 / 8$ \\
\hline Soriamys gaimanensis & $x$ & - & - & - & - & - & $1 / 6$ & $x$ & $x$ & $3 / 8$ \\
\hline Scleromys quadrangulatus & - & - & - & - & - & - & $0 / 6$ & - & $x$ & $1 / 8$ \\
\hline Scleromys osbornianus & $x$ & - & - & - & - & - & $1 / 6$ & - & - & $1 / 8$ \\
\hline Scleromys angustus & $x$ & - & - & $x$ & - & - & $2 / 6$ & - & - & $2 / 8$ \\
\hline Scleromys praecursor & $x$ & $x$ & - & $x$ & - & - & $3 / 6$ & $x$ & $x$ & $5 / 8$ \\
\hline Microscleromys paradoxalis-cribriphilus & $x$ & $x$ & - & $x$ & - & - & $3 / 6$ & $x$ & $x$ & $5 / 8$ \\
\hline Drytomomys aequatorialis & $x$ & - & - & - & - & - & $1 / 6$ & - & - & $1 / 8$ \\
\hline Potamarchus murinus & $x$ & - & - & $x$ & - & $x$ & $3 / 6$ & $x$ & - & $4 / 8$ \\
\hline Dinomys branickii & $x$ & - & - & $x$ & - & - & $2 / 6$ & - & - & $2 / 8$ \\
\hline Neoepiblema ambrosettianus-horridula & $x$ & - & - & $x$ & - & - & $2 / 6$ & - & - & $2 / 8$ \\
\hline Garridomys curunuquem & $x$ & - & - & $x$ & - & - & $2 / 6$ & $\times$ & $x$ & $4 / 8$ \\
\hline Eoviscaccia frassinettii & - & $x$ & $x$ & $x$ & - & - & $3 / 6$ & $x$ & $x$ & $5 / 8$ \\
\hline Eoviscaccia australis & - & - & - & - & - & - & $0 / 6$ & - & $x$ & $1 / 8$ \\
\hline Perimys intermedius & - & - & - & - & - & - & $0 / 6$ & $x$ & $x$ & $2 / 8$ \\
\hline Chinchilla lanigera & $x$ & - & - & $x$ & - & - & $2 / 6$ & - & - & $2 / 8$ \\
\hline Lagidium viscacia & $x$ & - & - & $x$ & - & - & $2 / 6$ & - & - & $2 / 8$ \\
\hline
\end{tabular}




\begin{tabular}{|c|c|c|c|c|c|c|c|c|c|c|}
\hline Taxons avec locus manquant & dP4 & P4 & M1-3 & dp4 & p4 & m1-3 & $\begin{array}{l}\text { Total loci } \\
\text { dentaire } \\
\text { manquant }\end{array}$ & 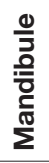 & $\begin{array}{l}\stackrel{0}{\frac{10}{0}} \\
\frac{\cos }{0}\end{array}$ & $\begin{array}{c}\text { Total } \\
\text { structure } \\
\text { manquante }\end{array}$ \\
\hline Lagostomus maximus & $x$ & - & - & $x$ & - & - & $2 / 6$ & - & - & $2 / 8$ \\
\hline Eoespina woodi-Eosachacui lavocati & - & - & - & - & - & - & $0 / 6$ & - & $x$ & $1 / 8$ \\
\hline Draconomys verai & $x$ & $x$ & - & $x$ & - & - & $3 / 6$ & - & $x$ & $4 / 8$ \\
\hline Platypittamys brachyodon & $x$ & - & - & $x$ & - & - & $2 / 6$ & - & - & $2 / 8$ \\
\hline Eosallamys paumacoutoi-simpsoni & $x$ & - & - & - & $x^{*}$ & - & $2 / 6$ & $x$ & $x$ & $4 / 8$ \\
\hline Prospaniomys priscus-Prospaniomys cf. priscus & - & + & - & - & + & - & $2 / 6$ & - & - & $2 / 8$ \\
\hline Spaniomys riparius & - & + & - & - & + & - & $2 / 6$ & - & - & $2 / 8$ \\
\hline Abrocoma bennettii & $x$ & $x$ & $x$ & $x$ & $x$ & - & $4 / 6$ & - & - & $4 / 8$ \\
\hline Protadelphomys latus & $x$ & - & - & $x$ & - & - & $2 / 6$ & $x$ & - & $3 / 8$ \\
\hline Willidewu esteparius & $x$ & - & - & $x$ & - & - & $2 / 6$ & - & - & $2 / 8$ \\
\hline Xenodontomys simpsoni-elongatus & - & + & - & - & + & - & $2 / 6$ & - & - & $2 / 8$ \\
\hline Ctenomys boliviensis & - & + & - & - & + & - & $2 / 6$ & - & - & $2 / 8$ \\
\hline Caviocricetus lucasi & - & + & - & - & + & - & $2 / 6$ & - & $x$ & $3 / 8$ \\
\hline Plesiacarechimys koenigswaldi & - & + & - & - & + & - & $2 / 6$ & - & $x$ & $3 / 8$ \\
\hline Dudumus ruigomezi & - & + & - & - & + & - & $2 / 6$ & - & $x$ & $3 / 8$ \\
\hline Galileomys eurygnathus & - & - & - & - & - & - & $0 / 6$ & - & $x$ & $1 / 8$ \\
\hline Neophanomys biplicatus & $x$ & + & $x$ & - & + & - & $4 / 6$ & $x$ & $x$ & $6 / 8$ \\
\hline Pithanotomys columnaris & - & + & - & - & + & - & $2 / 6$ & - & - & $2 / 8$ \\
\hline Octodon degus & - & + & - & - & + & - & $2 / 6$ & - & - & $2 / 8$ \\
\hline Spalacopus cyanus & - & + & - & - & + & - & $2 / 6$ & - & - & $2 / 8$ \\
\hline Mesocapromys/Mysasteles sp. & $x$ & $x$ & - & $x$ & $x$ & - & $4 / 6$ & - & - & $4 / 8$ \\
\hline Myocastor coypus & - & + & - & - & + & - & $2 / 6$ & - & - & $2 / 8$ \\
\hline Deseadomys arambourgi & $x$ & - & - & $x$ & - & - & $2 / 6$ & - & $x$ & $3 / 8$ \\
\hline Adelphomys candidus & $x$ & + & - & - & + & - & $3 / 6$ & - & $x$ & $4 / 8$ \\
\hline Prostichomys bowni & - & + & - & - & + & - & $2 / 6$ & - & $x$ & $3 / 8$ \\
\hline Stichomys regularis & - & + & - & - & + & - & $2 / 6$ & - & - & $2 / 8$ \\
\hline Maruchito trilofodonte & $x$ & $x$ & - & - & $x$ & - & $3 / 6$ & - & $x$ & $4 / 8$ \\
\hline Proechimys cuvieri & - & + & - & - & + & - & $2 / 6$ & - & - & $2 / 8$ \\
\hline Echimys chrysurus & - & + & - & - & + & - & $2 / 6$ & - & - & $2 / 8$ \\
\hline Makalata didelphoides & - & + & - & - & + & - & $2 / 6$ & - & - & $2 / 8$ \\
\hline Pampamys emmonsae & - & + & - & - & + & - & $2 / 6$ & - & $x$ & $3 / 8$ \\
\hline Thrichomys apereoides & - & + & - & - & + & - & $2 / 6$ & - & - & $2 / 8$ \\
\hline Euryzygomatomys spinosus & - & + & - & - & + & - & $2 / 6$ & - & - & $2 / 8$ \\
\hline Loretomys minutus & $x$ & $x$ & - & $x$ & $x$ & $x$ & $5 / 6$ & $x$ & $x$ & $7 / 8$ \\
\hline Mayomys confluens & - & $x$ & - & - & - & - & $1 / 6$ & $x$ & $x$ & $3 / 8$ \\
\hline Leucokephalos zeffiae & $x$ & $x$ & $x$ & $x$ & - & - & $4 / 6$ & - & $x$ & $5 / 8$ \\
\hline Pozomys ucayaliensis & $x$ & $x$ & - & $x$ & - & - & $3 / 6$ & - & $x$ & $4 / 8$ \\
\hline Chambiramys sylvaticus & - & $x$ & - & $x$ & - & - & $2 / 6$ & $x$ & $x$ & $4 / 8$ \\
\hline Chambiramys shipiborum & - & $x$ & - & - & - & - & $1 / 6$ & $x$ & $x$ & $3 / 8$ \\
\hline Tarapotomys mayoensis & - & - & - & - & - & - & $0 / 6$ & $x$ & $x$ & $2 / 8$ \\
\hline Tarapotomys subandinus & - & - & - & $x$ & $x$ & - & $2 / 6$ & $x$ & $x$ & $4 / 8$ \\
\hline
\end{tabular}

typeset $=O R D$ dans PAUP). Pour équilibrer l'influence de ces caractères en termes de changements évolutifs par rapport aux caractères non-ordonnés (binaires ou à états multiples non additifs), les caractères à états multiples additifs ont été équilibrés (commande wtset ${ }^{*}$ saled dans PAUP): le nombre de changements d'états de caractères a été pris en compte afin que la somme des changements (nombre de pas) suivant l'ordination soit égale à «1». Aucune pondération différentielle a priori entre caractères n'a été appliquée. L'option d'optimisation ACCTRAN (Accelerated transformation) a été choisie pour chacune des analyses.

La variabilité intraspécifique et, dans certain cas, intragénérique (voir section "Choix des taxons") a été considérée comme des états polymorphes (polymorph; état $\mathrm{x}$ et état $\mathrm{y}$ ) et ont été notés $\mathrm{x}+\mathrm{y}$ sous NDE et (xy) sous PAUP. Ils sont au nombre de 1547 sur un total de 59508 états soit 2,6\%. Bien plus rares (18 soit $0,03 \%$ des états), les états intermédiaires (uncertain; état $\mathrm{x}$ ou état $\mathrm{y}$ ) ont été notés $\mathrm{x} / \mathrm{y}$ sous NDE et $\{x y\}$ sous PAUP. Au vu du grand nombre d'états polymorphes par rapport aux états intermédiaires, seul le polymorphisme a été prise en compte (commande options mstaxa=polymorph).

Les états manquants et non-applicables ont été respectivement codés par «?» et «-». Certains taxons, notamment parmi les formes actuelles, présentent de nombreux états ambigus (codés «?» ou «-» selon les cas; Annexe 2). Ces derniers résultent souvent de la présence chez ces formes d'un patron dentaire dérivé sur lequel la reconnaissance des structures homologues est difficile. Les caractères correspondant à la taille relative d'une dent par rapport à un autre locus ne sont pas pris en compte (codés «?») chez les taxons connus uniquement par du matériel isolé pour ces loci.

La polarisation des caractères a été réalisée a posteriori grâce au critère de comparaison extra-groupe (Watrous \& Wheeler 1981 ; Darlu \& Tassy 1993; voir section «Choix des taxons" pour le choix de l'extra-groupe). 


\section{ChOIX DES TAXONS}

Un total de 116 taxons, dont 91 éteints et 25 modernes, a été considéré dans ces analyses (Annexe 3). Les taxons ont été majoritairement codés au niveau spécifique. Pour neuf taxons fossiles, deux espèces (voire trois pour Tamquammys) ont toutefois été considérées, pour améliorer le degré de complétude de leur codage. Toutefois, cette procédure pose question quant au traitement des caractères variables entre espèces d'un même genre (liés à la variabilité intragénérique). Ne sachant pas quel est l'état ancestral de ces caractères chez les genres concernés, ils ont été codés comme de la variabilité spécifique (état $\mathrm{x}$ et état $\mathrm{y}$ ) ce qui a sans doute contribué à augmenter artificiellement le codage du "polymorphisme» de ces taxons. Du fait d'une synonymie supposée entre Eoespina woodi et Eosachacui lavocati de Santa Rosa (Boivin et al. 2017a), ces deux espèces ont été codées comme s'il s'agissait d'un seul taxon (i.e., Eoespina woodi-Eosachacui lavocati). Bien qu'une priorité ait été donnée à l'observation des espèces types de chaque genre actuel, leur sélection a été tributaire du matériel disponible en musée et notamment de l'observation, en plus de spécimens adultes, de juvéniles/subadultes caractérisés par des dents peu usées, permettant ainsi de vérifier un maximum de caractères. L'attribution spécifique du matériel observé en collection reste indéterminée pour deux taxons modernes: Dasyprocta sp. et Mesocapromys/Mysateles sp. Ce dernier a été codé à partir d'un seul crâne. En revanche, le codage de Dasyprocta a été réalisé à partir de plusieurs crânes (Dasyprocta sp.), ce qui a pu générer un plus grand nombre de caractères "polymorphes" pour ce taxon (là encore liés à de la variabilité intragénérique).

L'extra-groupe inclut un glire basal (Tribosphenomys) et deux rongeurs basaux (Archetypomys et Cocomys). Le groupe de branchement (voir Antoine 2002, 2003 et Orliac et al. 2010) comprend un ischyromyoïde (Reithroparamys) et 16 Ctenohystrica: trois ctenohystricas basaux ("Ctenodactyloidea": Birbalomys, Chapattimys et Tamquammys), quatre hystricognathes asiatiques ("Baluchimyinae»: Baluchimys, Bugtimys, Hodsahibia et Ottomania) et neuf hystricognathes africains (hystricognathe basal: "Waslamys»; Gaudeamuridae: Gaudeamus aslius, G. hylaeus et G. aegyptius; "phiomorphes»: Acritophiomys, Phiomys, Metaphiomys, Paraphiomys et Thryonomys). Aucun hystricidé (actuel ou fossile) n'a été inclus dans les analyses en raison de leur registre ancien peu connu (Sallam \& Seiffert 2016) et de leur position phylogénétique ambiguë (Sallam et al. 2009, 2011; Coster et al. 2010, 2012; Barbière \& Marivaux 2015). De même, les Ctenodactylidae (et les Diatomyidae) n'ont pas été pris en compte en raison de leur morphologie dentaire très dérivée et convergente avec certains octodontoïdes (e.g. octodontidés, cténomyidés). Quatre-vingt seize caviomorphes constituent le groupe interne: 35 sont paléogènes (dont 17 espèces de Contamana et de Tarapoto), 36 miocènes, un pliocène et 24 actuels. Le choix des taxons du groupe interne s'est opéré en privilégiant les taxons bien documentés et dont le matériel a été directement observé. Au moins un représentant actuel par famille et sous-famille a été inclus dans cette analyse. Enfin, il est à noter que 79 taxons du groupe interne ne sont pas documentés pour tous les loci dentaires. Ce chiffre notable est toutefois biaisé par la prise en considération de 21 octodontoïdes, lesquels sont caractérisés par la rétention des $\mathrm{dp} 4 / \mathrm{dP} 4$ à l'âge adulte (i.e., les prémolaires définitives n'existent pas, d'où l'absence de codage sur ces dernières) (Tableau 1). Si l'on ne tient pas compte des caractères localisés sur $\mathrm{p} 4$ et $\mathrm{P} 4$ pour les taxons retenant leurs $\mathrm{dp} 4 / \mathrm{dP} 4$ à l'âge adulte, le nombre de taxons du groupe interne qui ne sont pas documentés par tous les loci dentaires est alors réduit à 60 .

\section{TRAITEMENT DES DONNÉES}

Les reconstructions phylogénétiques ont été exécutées par le logiciel PAUP* v.4.0 beta 10 Win (Swofford 2002). Au vu du grand nombre de taxons, une méthode de recherche heuristique (commande Hsearch) a été choisie, avec 1000 réplications et une addition aléatoire de séquences taxonomiques imposée à chaque réplication. La majorité des analyses ont été réalisées sur le cluster de calcul MBB (Montpellier Bioinformatics Biodiversity computing cluster platform, ISE-M, Montpellier, France). La visualisation et l'analyse des résultats ont été effectuées avec FigTree v.1.4.2 (Rambaut 2014) et Mesquite v.2.72 (Maddison \& Maddison 2009). Afin de tester le soutien des clades obtenus, les indices de Bremer (Bremer 1994) ont été calculés avec PAUP.

Une contrainte topologique des espèces actuelles, a été appliquée pour les analyses AG1-AG9 (commandes loadconstr asbackbone et enforce dans PAUP; Springer et al. 2001; voir section "Analyses phylogénétiques»). Elle est basée sur des résultats robustes de phylogénies moléculaires (ARNr 12S, cyt- $b$, GHR, RAGI, vWF; Upham \& Patterson 2015). Elle permet de conserver les clades de caviomorphes fortement soutenus par les données génétiques. Cette contrainte reconnaît la monophylie des quatre super-familles de caviomorphes (Octodontoidea, Cavioidea, Erethizontoidea et Chinchilloidea) et soutient d'étroites relations de parenté, d'une part entre les cavioïdes et les éréthizontoïdes, et d'autre part entre les chinchilloïdes et les octodontö̈des. Des relations interfamiliales et intergénériques au sein des super-familles sont également soutenues. L'arbre de contraintes employé dans la majorité des analyses (AG1-AG3, AG6, AG8 et AG9) est illustré en Figure 3. Pour les AG4, AG5 et AG7, des contraintes, légèrement modifiées par rapport à celle de la Figure 3, ont été appliquées (voir section «Autres analyses contraintes (de l'AG2 à l'AG9)»). Dans ce contexte phylogénétique où les relations entre les taxons actuels sont préétablies par des données génétiques, les nombreux taxons fossiles sélectionnés pour notre analyse cladistique, non pris en compte par la contrainte, se positionnent par rapport aux espèces actuelles en fonction de leurs caractéristiques morphologiques.

\section{ANALYSES PHYLOGÉNÉTIQUES}

Plusieurs analyses phylogénétiques avec un nombre variable de taxons ont été réalisées (AG0-AG9; Tableau 2). Dans aucune de ces analyses, les 116 taxons codés n’ont été considérés tous ensembles. Au maximum 111 taxons ont été inclus dans une même analyse (AG3-AG5).

Les analyses effectuées (Tableau 2) sont: 


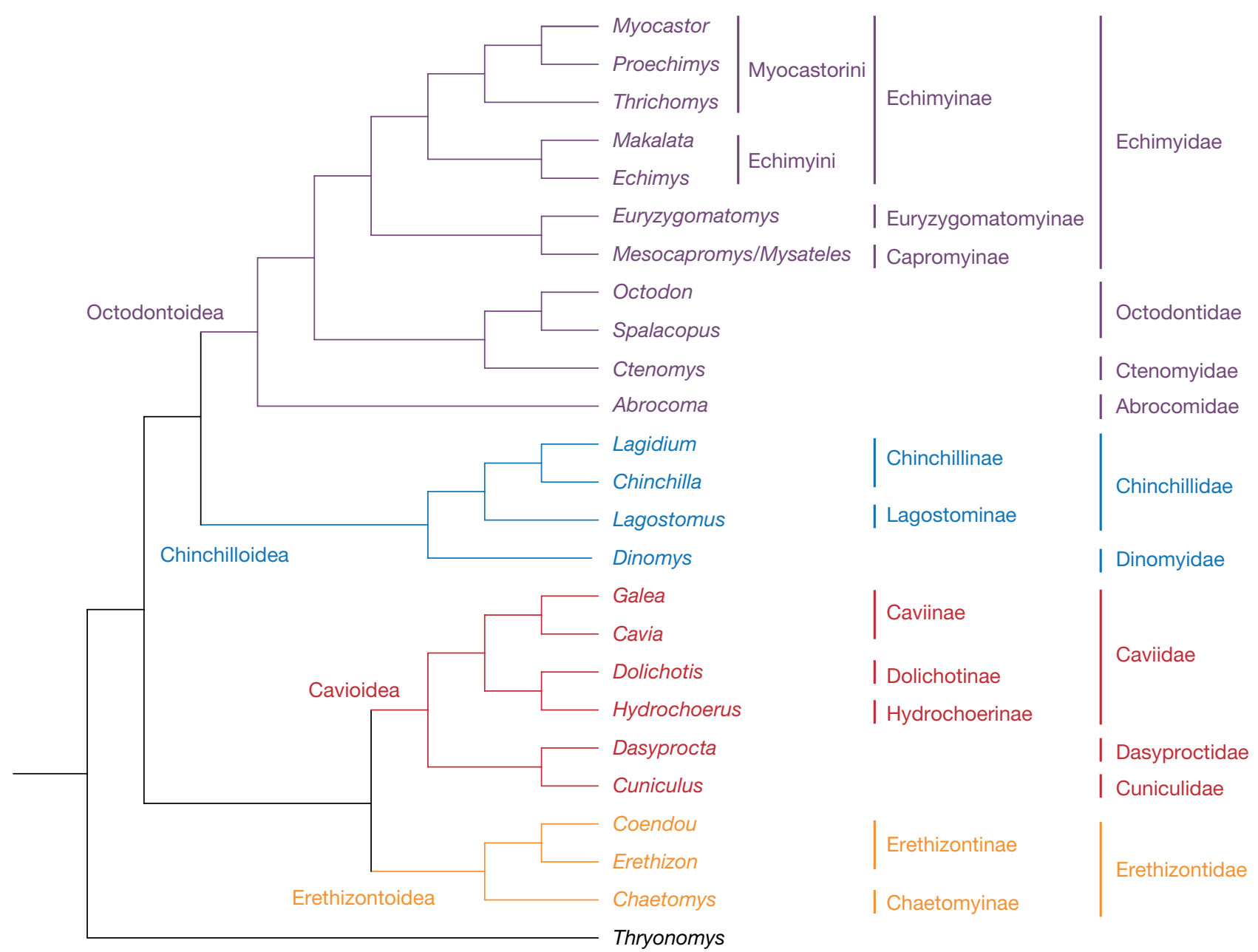

FIG. 3. - Arbre de contrainte employé pour les AG1-AG9, basé sur des données moléculaires. Modifié d'après Upham \& Patterson (2015: 77, fig. 3). Les quatre super-familles de caviomorphes sont différenciées par des couleurs: Cavioidea (rouge), Chinchilloidea (bleu), Erehizontoidea (orange) et Octodontoidea (violet).

- une analyse principale, non contrainte (AG0), comprenant une grande majorité des taxons (107/116). Toutefois neuf taxons ont été exclus : les trois espèces de Gaudeamus du groupe de branchement, l'octodontoïde Loretomys, l'éréthizontoïde supposé Plesiosteiromys, les céphalomyidés Cephalomys arcidens et Soriamys, ainsi que les caviomorphes aux relations suprafamiliales incertaines comme Litodontomys et «Asteromys» bolivianus (Annexe 3). En effet, ces taxons étant généralement peu documentés (e.g. Plesiosteiromys, Loretomys et Litodontomys) ou ayant un statut suprafamilial ambigu du fait notamment de plusieurs caractères convergents partagés avec différents représentants des super-familles (i.e., cas des céphalomyidés, de Litodontomys et d' "Asteromys» bolivianus), leur inclusion conjointe dans les analyses entraîne visiblement de nombreuses irrésolutions topologiques. Le traitement de ces taxons a fait l'objet d'autres analyses, lesquelles seront décrites ci-dessous;

- une analyse principale contrainte qui comprend les mêmes taxons que l'AG0. Cette analyse a été divisée en deux sousanalyses: pour la première, les caractères ordonnés ont été équilibrés (AG1) au contraire de la seconde où les caractères sont restés non équilibrés (AG1 non équilibrée);
- les AG2-AG9 sont des analyses contraintes qui comprennent l'ensemble des taxons de l'AG0 et de l'AG1 et qui incluent successivement les différents taxons non pris en compte dans ces dernières analyses. Le cas de l'énigmatique hystricognathe africain Gaudeamus a été traité à part. Ce genre, illustrant une famille monogénérique du Paléogène (i.e., Gaudeamuridae Sallam et al. 2011) était communément rapproché de Thryonomys (Thryonomyidae) ou plus récemment d'Hystrix + Atherurus (i.e., Hystricidae; Sallam et al. 2009, 2011; Barbière \& Marivaux 2015). Cependant, certaines analyses cladistiques récentes soutiennent, en revanche, une affiliation aux caviomorphes (Coster et al. 2010, 2012; Sallam et al. 2011; Antoine et al. 2012). Gaudeamus est ici représenté par trois espèces ( $G$. aslius, $G$. hylaeus et $G$. aegyptius), toutes incluses dans une même analyse (AG2). Les céphalomyidés (Cephalomys arcidens et Soriamys) et les taxons rapprochés de cette famille (Litodontomys et "Asteromys» bolivianus) ont été également traités à part et et ont fait l'objet de plusieurs analyses (AG3-AG7). Enfin, l'ajout de Plesiosteiromys et de Loretomys a fait l'objet de deux analyses séparées: AG8 et AG9, respectivement. 


\begin{tabular}{|c|c|c|c|c|c|c|c|c|c|c|c|}
\hline Analyses & $\begin{array}{l}\text { Nombre } \\
\text { total de } \\
\text { taxons } \\
\text { inclus }\end{array}$ & $\begin{array}{l}\text { Taxons } \\
\text { supplémentaires } \\
\text { par rapport à } \\
\text { l'AGO }\end{array}$ & 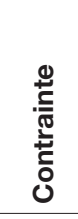 & 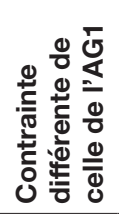 & 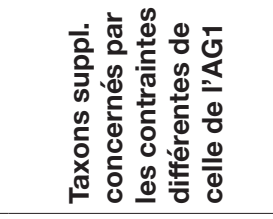 & $\begin{array}{l}\text { Type des } \\
\text { contraintes } \\
\text { différentes } \\
\text { de celle } \\
\text { de l'AG1 } \\
\end{array}$ & 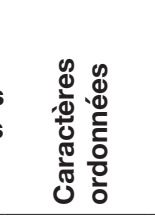 & 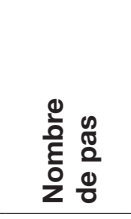 & 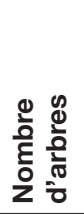 & IC & IR \\
\hline AGO & 107 & - & Non & - & - & - & équilibrés & 3274,16 & 5 & 0,578 & 0,607 \\
\hline AG1 équilibré & 107 & 0 & Oui & - & - & - & équilibrés & 3302,65 & 8 & 0,573 & 0,599 \\
\hline $\begin{array}{l}\text { AG1 non } \\
\text { équilibré }\end{array}$ & 107 & 0 & Oui & - & - & - & $\begin{array}{l}\text { non } \\
\text { équilibrés }\end{array}$ & 3839 & 872 & 0,547 & 0,593 \\
\hline AG2 & 110 & $\begin{array}{c}\text { Gaudeamus aslius, } \\
\text { G. hylaeus et } \\
\text { G. aegyptius }\end{array}$ & Oui & Non & - & - & équilibrés & 3493,48 & 42 & 0,582 & 0,597 \\
\hline AG3 & 111 & $\begin{array}{l}\text { "Asteromys" } \\
\text { bolivianus, } \\
\text { Cephalomys } \\
\text { arcidens, } \\
\text { Litodontomys et } \\
\text { Soriamys }\end{array}$ & Oui & Non & - & - & équilibrés & 3393,98 & 2 & 0,562 & 0,592 \\
\hline AG4 & 111 & $\begin{array}{l}\text { "Asteromys" } \\
\text { bolivianus, } \\
\text { Cephalomys } \\
\text { arcidens, } \\
\text { Litodontomys et } \\
\text { Soriamys }\end{array}$ & Oui & Oui & $\begin{array}{l}\text { "Asteromys» } \\
\text { bolivianus, } \\
\text { Asteromys punctus, } \\
\text { C. arcidens, } \\
\text { Litodontomys et } \\
\text { Soriamys }\end{array}$ & $\begin{array}{l}\text { Clade } \\
\text { dans les } \\
\text { chinchilloïdes }\end{array}$ & équilibrés & 3397,47 & 291 & 0,511 & 0,591 \\
\hline AG5 & 111 & $\begin{array}{l}\text { "Asteromys" } \\
\text { bolivianus, } \\
\text { Cephalomys } \\
\text { arcidens, } \\
\text { Litodontomys et } \\
\text { Soriamys }\end{array}$ & Oui & Oui & $\begin{array}{l}\text { "Asteromys" } \\
\text { bolivianus, } \\
\text { A. punctus, } \\
\text { C. arcidens, } \\
\text { Litodontomys et } \\
\text { Soriamys }\end{array}$ & $\begin{array}{l}\text { Clade } \\
\text { dans les } \\
\text { cavioïdes }\end{array}$ & équilibrés & 3398,14 & 6 & 0,561 & 0,591 \\
\hline AG6 & 110 & $\begin{array}{l}\text { «Asteromys» } \\
\text { bolivianus, } \\
\text { Cephalomys } \\
\text { arcidens et } \\
\text { Litodontomys }\end{array}$ & Oui & Non & - & - & équilibrés & 3372,97 & 4 & 0,565 & 0,594 \\
\hline AG7 & 110 & $\begin{array}{l}\text { «Asteromys» } \\
\text { bolivianus, } \\
\text { Cephalomys } \\
\text { arcidens et } \\
\text { Litodontomys }\end{array}$ & Oui & Oui & $\begin{array}{l}\text { "Asteromys» } \\
\text { bolivianus, } A \text {. } \\
\text { punctus, C. arcidens } \\
\text { et Litodontomys }\end{array}$ & $\begin{array}{l}\text { Clade } \\
\text { dans les } \\
\text { chinchilloïdes }\end{array}$ & équilibrés & 3373,31 & 52 & 0,565 & 0,594 \\
\hline AG8 & 108 & Plesiosteiromys & Oui & Non & - & - & équilibrés & 3304,98 & 16 & 0,573 & 0,599 \\
\hline AG9 & 108 & Loretomys & Oui & Non & - & - & équilibrés & 3308,15 & 54 & 0,573 & 0,599 \\
\hline
\end{tabular}

\section{ABRÉVIATIONS}

INSTITUTIONNELLES

LACM Los Angeles County Museum, Los Angeles;

MACN Museo Argentino de Ciencias Naturales «Bernardino Rivadavia", Buenos Aires;

MUSM Museo de Historia Natural de la Universidad Nacional Mayor San Marcos, Lima.

\section{RÉSULTATS}

Dans cette section, les topologies obtenues et les caractères/ états (car. Xétat) soutenant les principaux nœuds sont discutés. Pour la description de chaque nœud d'intérêt, les caractères ambigus sont notés " $A$ " et les caractères nonambigus "NA», et pour chacun leur nombre est indiqué entre parenthèses.

\section{ANALYSE PRINCIPALE NON CONTRAINTE (AG0)}

Les résultats de l'AG0 sont disponibles en Figure 4 (cinq arbres équiparcimonieux de 3274,16 pas chacun; Indice de Cohérence $[\mathrm{IC}]=0,578$; Indice de Rétention $[\mathrm{IR}]=0,607)$. Compte tenu du nombre de taxons (>100), les indices ont une valeur élevée, qui témoigne d'une homoplasie à la fois modérée et structurante au sein de l'échantillon. Ici, seul un consensus strict est présenté où les hypothèses de parenté sont à la fois très peu ambiguës mais peu en accord avec les données moléculaires. En effet, plusieurs relations de parenté intra- et inter-suprafamiliales appuyées par les données moléculaires (concernant les taxons modernes sélectionnés) ne sont pas soutenues. Par exemple, pour les relations entre les super-familles, les chinchilloïdes et les octodontoïdes sont monophylétiques mais leur position de groupe-frère n'est pas retrouvée. De plus, les éréthizontoïdes et cavioïdes sont paraphylétiques, Chaetomys s'associant avec Cuniculus. 


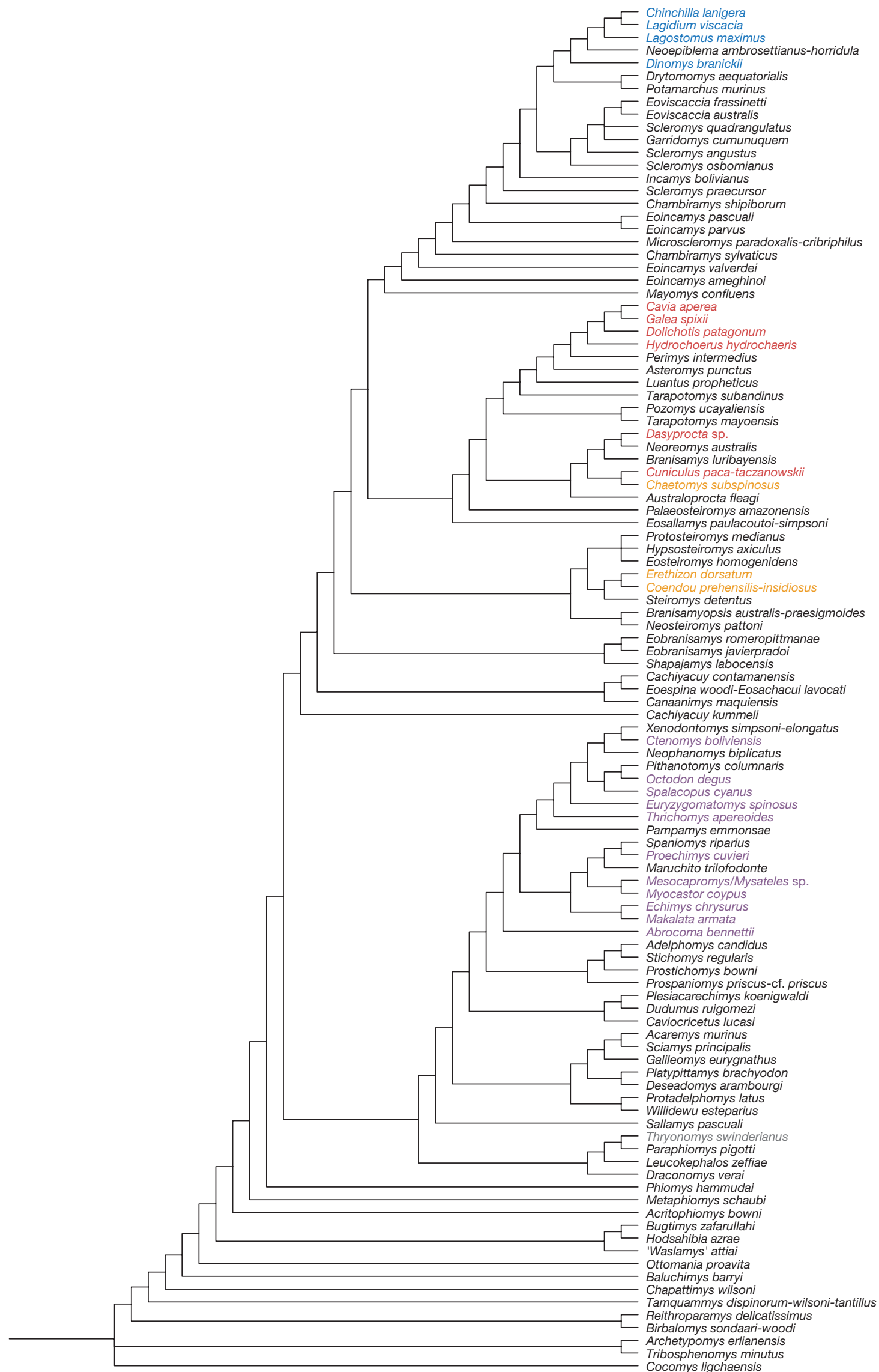

FIG. 4. - Résultats de l'AG0. Consensus strict de cinq arbres équiparcimonieux de 3274,16 pas chacun (IC =0,578; IR =0,607). Le code couleur est le même que celui de la Figure 3 . Ici, seulement les taxons actuels ont été colorés. 
ANALYSE PRINCIPALE CONTRAINTE

(AG1 ET AG1 NON ÉQUILIBRÉE)

$A G 1$

Les analyses heuristiques effectuées sur le jeu de données taxons/caractères de l'AG1 ont généré huit arbres équiparcimonieux de 3302,65 pas chacun $(\mathrm{IC}=0,573$; IR = 0,599). L'arbre de consensus strict (3306,65 pas) est présenté en Figure 5. Les indices de Bremer de l'ensemble des nœuds du consensus strict de l'AG1 sont disponibles en Figure 6. La liste des caractères aux nouds est donnée en Annexe 5 et la liste des ICs et IRs de chaque caractère est donnée dans le Tableau 2. En comparaison avec les résultats de l'AG0, la contrainte appliquée au jeu de données dans l'AG1 se traduit par une augmentation du nombre de pas $(28,49$ pas de différence) mais n'implique pas de changements drastiques sur l'homoplasie générée. Bien que la topologie de certains clades soit identique entre les deux analyses (e.g. Chinchilloidea), les consensus stricts de l'AG0 et AG1 varient sensiblement. Les résultats de l'AG1 stipulent que l'homoplasie est significative mais reste, cependant, minoritaire. En effet, la majorité des caractères utilisés $(68 \%)$ présentent un IC (ainsi qu'un IR) strictement supérieur à 0,500, et 38\% des caractères ont un IC supérieur à 0,700 (43\% ont un IR supérieur à 0,700 ; Tableau 3). De plus, l'examen approfondi des caractères fortement homoplasiques (i.e., IC $\leq 0,500$ ) révèle une structuration dans leur distribution interne (i.e., IR > 0,500) pour 45\% d'entre eux.

Sur la base des résultats de l'AG1, 20 nouds principaux (de A à T) concernant les relations du groupe de branchement et du groupe d'étude seront ici passés en revue, ainsi que 31 nœuds associés aux super-familles de caviomorphes (N1 et N2 pour les Erethizontoidea, O1-O6 pour les Cavioidea, S1-S1 1 pour les Chinchilloidea, et T1-T12 pour les Octodontoidea). Le choix de ces 51 nœuds décrits s'est fait selon trois critères: i) leur correspondance à un niveau taxonomique supragénérique (i.e., groupe couronne, infra-ordre, parvordre, superfamille, famille, sous-famille, tribu); ii) s'ils concernent les espèces fossiles de Contamana et de Tarapoto/Shapaja; et iii) s'ils nécessitent d'être discutés par rapport à des études précédentes (voir section «Discussion»).

Nœud A - le clade des Hystricognathi (20 NA et 9 A). Ce clade extrêmement soutenu (i.e., supporté par 20 NA et indice de Bremer égal ou supérieur à 3) rassemble les «baluchimyinés" asiatiques (Baluchimys, Bugtimys, Hodsahibia et Ottomania), les hystricognathes africains (Acritophiomys, Metaphiomys, Paraphiomys, Phiomys, Thryonomys et "Waslamys») et l'ensemble des caviomorphes inclus dans l'analyse. Ces taxons sont notamment caractérisés par: des dents lophodontes (car. 12) avec des loph(-id)es hauts (car. 1281 et 2, 3641 et 3771) et montrant des connexions avec certaines cuspides (car. $132^{1}$ et 2221); une hauteur du talonide similaire à celle du trigonide sur les molaires inférieures (car. 3522); et la présence d'une troisième crête transverse sur les M1-2 (car. 1751). Les hystricognathes basaux montrent également un métaconule réduit (car. 1621) et mésialement déplacé (car. $163^{1}$ ) sur les molaires supérieures. Deux résultats de l'AG1 sont en accord avec des études récentes visant à reconstruire les relations phylogénétiques des hystricognathes (e.g. Marivaux et al. 2004; Antoine et al. 2012; Coster et al. 2012; Sallam et al. 2012; Barbière \& Marivaux 2015) : i) la paraphylie des «baluchimyinés » et des premiers hystricognathes africains; et ii) la divergence des caviomorphes à partir de certains hystricognathes africains basaux. Les relations entre caviomorphes et les hystricognathes africains (taxons basaux, Gaudeamuridae et "phiomorphes») qui leur sont étroitement apparentés semblent néanmoins varier par rapport aux analyses précédentes. Toutefois, il faut noter que les hystricognathes basaux de l'Ancien Monde (Asie et Afrique) n'ont pas été ici échantillonnés de façon exhaustive, et servent seulement de groupes de branchement aux caviomorphes (mais voir section "Autres analyses contraintes (de l'AG2 à l'AG9)»), ce qui limite la portée des comparaisons avec les études précédentes.

Noud B - le clade des Caviomorpha (10 NA et 11 A). Ce clade est très soutenu (i.e., soutenu par 10 NA et indice de Bremer égal ou supérieur à 3). Les caviomorphes regroupent: i) un grand clade incluant les quatre super-familles (Erethizontoidea, Cavioidea, Chinchilloidea et Octodontoidea) et les taxons basaux qui leur sont directement apparentés; et ii) une dizaine de taxons extérieurs à ce clade, situés à sa racine (branchements basaux: Cachiyacuy kummeli, C. contamanensis, Canaanimys, Eobranisamys, Eoespina, Pozomys, Shapajamys et Tarapotomys). La monophylie des caviomorphes est notamment soutenue par: la présence d'un mésostyle sur les M1-2 fortement développé en surface (car. 1661) et hauteur (car. 1671), et distalement déplacé (car. 1681), et un métacône réduit (car. 2011). Certains caviomorphes montrent des configurations accentuées de ces caractères: un mésostyle encore plus développé (car. $166^{2}$ et 1672 ) et déplacé distalement ( $168^{2}$; particulièrement chez les octodontoïdes), ainsi qu'un métacône très réduit à indistinct (2012).

Nœud C ( 1 NA et 1 A). Ce nœud correspond à l'ensemble des caviomorphes à l'exception de Cachiyacuy kummeli. Il n'est soutenu que par un seul NA (et $1 \mathrm{~A}$ ) : un protolophe lingual labiodistalement orienté sur la P4 (car. 382), tandis qu'il est transverse chez C. kummeli et les taxons du groupe de branchement codés pour ce caractère. En outre, le caractère 38 est très peu codé à l'échelle de la matrice (seulement chez 28 taxons).

Nœud D (1 NA et 8 A). Ce nœud correspond au clade divergeant de la majorité des caviomorphes et issu du noeud C. Il réunit Cachiyacuy contamanensis, Canaanimys et Eoespina, révèlant ainsi la paraphylie du genre Cachiyacuy. Un seul NA le soutient toutefois: un entoconide connecté postérieurement au postérolophide (car. 3411).

Noud E (1 NA et 1 A). Ce noud exprime la monophylie de Cachiyacuy contamanensis avec Eoespina, qui n'est elle-même soutenue que par un seul NA (et 1 A) : un métacône postérieurement isolé du postérolophide (car. 2080). 
TABLEAU 3. - Indices de Cohérence (IC) et de Rétention (IR) calculés pour chaque caractère (Car.) obtenus en AG1. Les caractères 140 et 233 étant constants, ils n'apparaissent pas dans ce tableau.

\begin{tabular}{|c|c|c|c|c|c|c|c|c|c|c|c|}
\hline Car. & IC & IR & Car. & IC & IR & Car. & IC & IR & Car. & IC & IR \\
\hline 1 & 0,67 & 0,89 & 75 & 0,60 & 0,00 & 150 & 0,73 & 0,82 & 224 & 0,20 & 0,00 \\
\hline 2 & 1,00 & 1,00 & 76 & 0,50 & 0,79 & 151 & 0,40 & 0,65 & 225 & 0,40 & 0,40 \\
\hline 3 & 0,27 & 0,77 & 77 & 0,75 & 0,67 & 152 & 0,67 & 0,67 & 226 & 0,14 & 0,54 \\
\hline 4 & 0,50 & 0,76 & 78 & 0,25 & 0,50 & 153 & 0,67 & 0,50 & 227 & 0,14 & 0,00 \\
\hline 5 & 0,57 & 0,71 & 79 & 0,75 & 0,00 & 154 & 0,70 & 0,77 & 228 & 0,40 & 0,80 \\
\hline 6 & 0,50 & 0,50 & 80 & 0,50 & 0,67 & 155 & 0,67 & 0,67 & 229 & 0,17 & 0,23 \\
\hline 7 & 0,50 & 0,00 & 81 & 1,00 & $0 / 0$ & 156 & 1,00 & $0 / 0$ & 230 & 0,50 & 0,67 \\
\hline 8 & 0,07 & 0,19 & 82 & 0,33 & 0,60 & 157 & 0,50 & 0,50 & 231 & 0,40 & 0,25 \\
\hline 9 & 0,11 & 0,77 & 83 & 1,00 & 1,00 & 158 & 1,00 & $0 / 0$ & 232 & 0,80 & 0,90 \\
\hline 10 & 0,25 & 0,87 & 84 & 0,47 & 0,63 & 159 & 0,67 & 0,78 & 234 & 0,29 & 0,74 \\
\hline 11 & 0,13 & 0,65 & 85 & 0,50 & 0,71 & 160 & 0,50 & 0,67 & 235 & 0,20 & 0,73 \\
\hline 12 & 0,25 & 0,63 & 86 & 1,00 & $0 / 0$ & 161 & 1,00 & 1,00 & 236 & 1,00 & 1,00 \\
\hline 13 & 0,67 & 0,91 & 87 & 0,50 & 0,77 & 162 & 1,00 & 1,00 & 237 & 1,00 & 1,00 \\
\hline 14 & 0,33 & 0,60 & 88 & 0,60 & 0,75 & 163 & 1,00 & 1,00 & 238 & 1,00 & 1,00 \\
\hline 15 & 1,00 & 1,00 & 89 & 0,75 & 0,50 & 164 & 0,63 & 0,00 & 239 & 1,00 & 1,00 \\
\hline 16 & 0,50 & 0,67 & 90 & 1,00 & 1,00 & 165 & 0,75 & 0,00 & 240 & 1,00 & 1,00 \\
\hline 17 & 0,54 & 0,75 & 91 & 0,50 & 0,00 & 166 & 0,55 & 0,85 & 241 & 0,75 & 0,75 \\
\hline 18 & 0,29 & 0,00 & 92 & 1,00 & 1,00 & 167 & 0,75 & 0,95 & 242 & 0,75 & 0,75 \\
\hline 19 & 0,25 & 0,40 & 93 & 0,83 & 0,67 & 168 & 0,38 & 0,82 & 243 & 0,33 & 0,33 \\
\hline 20 & 0,13 & 0,13 & 94 & 0,33 & 0,78 & 169 & 0,78 & 0,00 & 244 & 0,25 & 0,67 \\
\hline 21 & 0,33 & 0,33 & 95 & 1,00 & 1,00 & 170 & 1,00 & $0 / 0$ & 245 & 0,20 & 0,60 \\
\hline 22 & 0,15 & 0,35 & 96 & 0,38 & 0,52 & 171 & 0,64 & 0,50 & 246 & 0,50 & 0,67 \\
\hline 23 & 1,00 & $0 / 0$ & 97 & 0,38 & 0,44 & 172 & 1,00 & $0 / 0$ & 247 & 0,33 & 0,50 \\
\hline 24 & 0,50 & 0,67 & 98 & 0,40 & 0,50 & 173 & 0,52 & 0,29 & 248 & 0,67 & 0,83 \\
\hline 25 & 1,00 & 1,00 & 99 & 0,50 & 0,60 & 174 & 0,39 & 0,30 & 249 & 0,67 & 0,67 \\
\hline 26 & 0,67 & 0,75 & 100 & 0,43 & 0,75 & 175 & 0,33 & 0,75 & 250 & 1,00 & $0 / 0$ \\
\hline 27 & 0,44 & 0,17 & 101 & 1,00 & $0 / 0$ & 176 & 0,67 & 0,83 & 251 & 0,50 & 0,00 \\
\hline 28 & 0,50 & 0,25 & 102 & 0,86 & 0,67 & 177 & 0,58 & 0,80 & 252 & 0,17 & 0,50 \\
\hline 29 & 0,67 & 0,50 & 103 & 0,43 & 0,64 & 178 & 0,58 & 0,74 & 253 & 0,11 & 0,53 \\
\hline 30 & 0,33 & 0,50 & 104 & 1,00 & 1,00 & 179 & 0,68 & 0,47 & 254 & 0,38 & 0,29 \\
\hline 31 & 0,33 & 0,74 & 105 & 1,00 & $0 / 0$ & 180 & 0,80 & 0,87 & 255 & 0,67 & 0,00 \\
\hline 32 & 1,00 & 1,00 & 106 & 1,00 & 1,00 & 181 & 0,89 & 0,91 & 256 & 0,31 & 0,25 \\
\hline 33 & 0,33 & 0,33 & 107 & 1,00 & 1,00 & 182 & 0,63 & 0,14 & 257 & 0,33 & 0,33 \\
\hline 34 & 1,00 & $0 / 0$ & 108 & 1,00 & $0 / 0$ & 183 & 0,81 & 0,73 & 258 & 0,38 & 0,23 \\
\hline 35 & 0,45 & 0,50 & 109 & 0,33 & 0,39 & 184 & 1,00 & $0 / 0$ & 259 & 0,43 & 0,20 \\
\hline 36 & 0,25 & 0,65 & 110 & 0,67 & 0,67 & 185 & 0,69 & 0,62 & 260 & 0,46 & 0,14 \\
\hline 37 & 1,00 & 1,00 & 111 & 0,17 & 0,29 & 186 & 1,00 & $0 / 0$ & 261 & 0,53 & 0,46 \\
\hline 38 & 0,60 & 0,85 & 112 & 1,00 & 1,00 & 187 & 1,00 & $0 / 0$ & 262 & 0,59 & 0,46 \\
\hline 39 & 0,50 & 0,63 & 113 & 0,60 & 0,00 & 188 & 0,50 & 0,00 & 263 & 0,29 & 0,71 \\
\hline 40 & 1,00 & 1,00 & 114 & 0,38 & 0,44 & 189 & 1,00 & 1,00 & 264 & 0,50 & 0,67 \\
\hline 41 & 0,50 & 0,67 & 115 & 0,67 & 0,84 & 190 & 0,33 & 0,00 & 265 & 0,58 & 0,74 \\
\hline 42 & 0,67 & 0,50 & 116 & 0,67 & 0,00 & 191 & 0,29 & 0,00 & 266 & 0,85 & 0,71 \\
\hline 43 & 0,50 & 0,60 & 117 & 0,67 & 0,63 & 192 & 0,15 & 0,00 & 267 & 0,67 & 0,40 \\
\hline 44 & 0,64 & 0,00 & 118 & 0,80 & 0,71 & 193 & 1,00 & 1,00 & 268 & 0,25 & 0,00 \\
\hline 45 & 1,00 & 1,00 & 119 & 1,00 & 1,00 & 194 & 1,00 & 1,00 & 269 & 0,55 & 0,64 \\
\hline 46 & 0,71 & 0,00 & 120 & 0,13 & 0,07 & 195 & 1,00 & $0 / 0$ & 270 & 0,77 & 0,29 \\
\hline 47 & 0,60 & 0,50 & 121 & 0,13 & 0,29 & 196 & 0,77 & 0,71 & 271 & 1,00 & $0 / 0$ \\
\hline 48 & 1,00 & $0 / 0$ & 122 & 0,07 & 0,52 & 197 & 0,44 & 0,55 & 272 & 0,63 & 0,46 \\
\hline 49 & 0,50 & 0,00 & 123 & 0,15 & 0,71 & 198 & 0,77 & 0,77 & 273 & 0,86 & 0,67 \\
\hline 50 & 0,56 & 0,00 & 124 & 1,00 & $0 / 0$ & 199 & 0,44 & 0,75 & 274 & 1,00 & 1,00 \\
\hline 51 & 0,56 & 0,71 & 125 & 0,84 & 0,40 & 200 & 0,50 & 0,69 & 275 & 1,00 & 1,00 \\
\hline 52 & 0,64 & 0,50 & 126 & 1,00 & 1,00 & 201 & 0,40 & 0,63 & 276 & 0,71 & 0,00 \\
\hline 53 & 0,75 & 0,00 & 127 & 0,33 & 0,00 & 202 & 0,40 & 0,63 & 277 & 0,93 & 0,67 \\
\hline 54 & 0,50 & 0,50 & 128 & 0,75 & 0,95 & 203 & 0,39 & 0,50 & 278 & 1,00 & $0 / 0$ \\
\hline 55 & 1,00 & 1,00 & 129 & 0,25 & 0,25 & 204 & 0,71 & 0,00 & 279 & 0,50 & 0,50 \\
\hline 56 & 0,75 & 0,82 & 130 & 0,70 & 0,67 & 205 & 0,60 & 0,56 & 280 & 0,67 & 0,80 \\
\hline 57 & 0,69 & 0,56 & 131 & 0,50 & 0,83 & 206 & 0,46 & 0,53 & 281 & 1,00 & $0 / 0$ \\
\hline 58 & 0,33 & 0,50 & 132 & 1,00 & 1,00 & 207 & 0,75 & 0,00 & 282 & 0,50 & 0,33 \\
\hline 59 & 0,78 & 0,60 & 133 & 0,33 & 0,60 & 208 & 0,11 & 0,47 & 283 & 0,60 & 0,00 \\
\hline 60 & 0,50 & 0,75 & 134 & 1,00 & 1,00 & 209 & 0,33 & 0,00 & 284 & 0,47 & 0,55 \\
\hline 61 & 0,50 & 0,25 & 135 & 0,50 & 0,88 & 210 & 1,00 & 1,00 & 285 & 0,67 & 0,50 \\
\hline 62 & 0,44 & 0,64 & 136 & 1,00 & 1,00 & 211 & 0,65 & 0,84 & 286 & 0,57 & 0,25 \\
\hline 63 & 0,33 & 0,56 & 137 & 1,00 & 1,00 & 212 & 0,62 & 0,82 & 287 & 0,83 & 0,00 \\
\hline 64 & 0,22 & 0,36 & 138 & 0,50 & 0,50 & 213 & 0,67 & 0,00 & 288 & 1,00 & 1,00 \\
\hline 65 & 0,29 & 0,29 & 139 & 0,50 & 0,80 & 214 & 0,85 & 0,67 & 289 & 0,80 & 0,78 \\
\hline 66 & 0,50 & 0,50 & 141 & 0,50 & 0,75 & 215 & 0,87 & 0,77 & 290 & 0,50 & 0,86 \\
\hline 67 & 0,50 & 0,40 & 142 & 0,73 & 0,53 & 216 & 1,00 & $0 / 0$ & 291 & 0,25 & 0,50 \\
\hline 68 & 0,46 & 0,68 & 143 & 0,64 & 0,38 & 217 & 0,70 & 0,70 & 292 & 0,75 & 0,00 \\
\hline 69 & 0,62 & 0,62 & 144 & 1,00 & 1,00 & 218 & 1,00 & $0 / 0$ & 293 & 0,50 & 0,50 \\
\hline 70 & 0,33 & 0,33 & 145 & 1,00 & $0 / 0$ & 219 & 0,50 & 0,80 & 294 & 0,75 & 0,00 \\
\hline 71 & 1,00 & 1,00 & 146 & 0,81 & 0,74 & 220 & 1,00 & 1,00 & 295 & 1,00 & $0 / 0$ \\
\hline 72 & 1,00 & 1,00 & 147 & 0,64 & 0,77 & 221 & 1,00 & 1,00 & 296 & 0,17 & 0,60 \\
\hline 73 & 0,33 & 0,56 & 148 & 0,57 & 0,75 & 222 & 0,27 & 0,56 & 297 & 1,00 & 1,00 \\
\hline 74 & 1,00 & 1,00 & 149 & 0,80 & 0,00 & 223 & 0,33 & 0,29 & 298 & 1,00 & 1,00 \\
\hline
\end{tabular}

\begin{tabular}{ccc}
\hline Car. & IC & IR \\
\hline 299 & 0,50 & 0,00 \\
300 & 0,50 & 0,00 \\
301 & 1,00 & $0 / 0$ \\
302 & 0,50 & 0,67 \\
303 & 0,13 & 0,53 \\
304 & 1,00 & 1,00 \\
305 & 0,29 & 0,44 \\
306 & 0,50 & 0,50 \\
307 & 0,50 & 0,88 \\
308 & 0,27 & 0,27 \\
309 & 0,50 & 0,67 \\
310 & 0,60 & 0,75 \\
311 & 0,33 & 0,33 \\
312 & 0,50 & 0,63 \\
313 & 0,57 & 0,50 \\
314 & 0,50 & 0,33 \\
315 & 0,83 & 0,50
\end{tabular}

$\begin{array}{ll}0,50 & 0,33\end{array}$

$\begin{array}{lll}315 & 0,83 & 0,50 \\ 316 & 1,00 & 0 / 0\end{array}$

$\begin{array}{lll}317 & 0,57 & 0,50\end{array}$

$\begin{array}{lll}318 & 1,00 & 0 / 0\end{array}$

$\begin{array}{lll}319 & 1,00 \quad 0 / 0\end{array}$

$320 \quad 0,75 \quad 0,50$

$\begin{array}{lll}321 & 1,00 & 0 / 0\end{array}$

$322 \quad 0,60 \quad 0,00$

$\begin{array}{lll}323 & 0,80 & 0,67\end{array}$

$\begin{array}{lll}324 & 0,86 & 0,00\end{array}$

$\begin{array}{llll}325 & 0,40 & 0,33\end{array}$

$\begin{array}{lll}326 & 0,67 & 0,00\end{array}$

$\begin{array}{lll}327 & 0,25 & 0,40\end{array}$

$\begin{array}{llll}328 & 0,47 & 0,47\end{array}$

$\begin{array}{lll}329 & 0,53 & 0,39\end{array}$

$\begin{array}{lll}330 & 0,50 & 0,29\end{array}$

$\begin{array}{lll}331 & 1,00 & 1,00\end{array}$

$\begin{array}{lll}332 & 0,67 & 0,67\end{array}$

$\begin{array}{lll}333 & 0,50 & 0,36\end{array}$

$\begin{array}{lll}334 & 0,63 & 0,36\end{array}$

$\begin{array}{lll}335 & 1,00 & 0 / 0\end{array}$

$\begin{array}{lll}336 & 1,00 & 1,00\end{array}$

$337 \quad 1,00 \quad 1,00$

$\begin{array}{llll}338 & 0,31 & 0,40\end{array}$

$\begin{array}{lll}339 & 0,63 & 0,25\end{array}$

$\begin{array}{lll}340 & 0,41 & 0,60\end{array}$

$\begin{array}{lll}341 & 0,85 & 0,60\end{array}$

$3421,00 \quad 1,00$

$\begin{array}{lll}343 & 0,60 & 0,33\end{array}$

$\begin{array}{llll}344 & 0,75 & 0,88\end{array}$

$\begin{array}{llll}345 & 0,80 & 0,67\end{array}$

$3461,00 \quad 1,00$

$\begin{array}{lll}347 & 0,50 & 0,63\end{array}$

$\begin{array}{lll}348 & 1,00 & 0 / 0\end{array}$

$\begin{array}{lll}349 & 0,50 & 0,83\end{array}$

$\begin{array}{lll}350 & 1,00 & 1,00\end{array}$

$\begin{array}{lll}351 & 1,00 & 1,00\end{array}$

$\begin{array}{lll}352 & 0,67 & 0,88\end{array}$

$\begin{array}{llll}353 & 0,13 & 0,22\end{array}$

$\begin{array}{lll}354 & 0,36 & 0,33\end{array}$

$\begin{array}{lll}355 & 0,80 & 0,80\end{array}$

$\begin{array}{lll}356 & 0,67 & 0,60\end{array}$

$\begin{array}{lll}357 & 0,23 & 0,50\end{array}$

$\begin{array}{lll}358 & 0,33 & 0,67\end{array}$

$\begin{array}{lll}359 & 0,50 & 0,56\end{array}$

$\begin{array}{lll}360 & 0,65 & 0,30\end{array}$

$\begin{array}{llll}361 & 0,60 & 0,00\end{array}$

$\begin{array}{lll}362 & 1,00 & 0 / 0\end{array}$

$\begin{array}{lll}363 & 0,67 & 0,00\end{array}$

$\begin{array}{lll}364 & 1,00 & 1,00\end{array}$

$\begin{array}{lll}365 & 1,00 & 0 / 0\end{array}$

$\begin{array}{lll}366 & 0,75 & 0,83\end{array}$

$\begin{array}{lll}367 & 0,84 & 0,40\end{array}$

$\begin{array}{lll}368 & 0,60 & 0,00\end{array}$

$\begin{array}{lll}369 & 0,97 & 0,67\end{array}$

$\begin{array}{llll}370 & 0,67 & 0,50\end{array}$

$\begin{array}{llll}371 & 0,71 & 0,78\end{array}$

$\begin{array}{llll}372 & 0,73 & 0,22\end{array}$

\section{Car. IC IR}

$\begin{array}{lll}373 & 0,91 & 0,80\end{array}$

$\begin{array}{llll}374 & 0,38 & 0,84\end{array}$

$\begin{array}{lll}375 & 0,35 & 0,45\end{array}$

$\begin{array}{llll}376 & 0,64 & 0,50\end{array}$

$\begin{array}{lll}377 & 1,00 & 1,00\end{array}$

$\begin{array}{lll}378 & 0,63 & 0,80\end{array}$

$\begin{array}{lll}379 & 1,00 & 1,00\end{array}$

$380 \quad 1,00 \quad 0 / 0$

$381 \quad 1,00 \quad 0 / 0$

$382 \quad 1,00 \quad 0 / 0$

$\begin{array}{llll}383 & 0,77 & 0,71\end{array}$

$\begin{array}{lll}384 & 0,70 & 0,33\end{array}$

$\begin{array}{lll}385 & 1,00 & 0 / 0\end{array}$

$\begin{array}{lll}386 & 0,86 & 0,83\end{array}$

$\begin{array}{lll}387 & 1,00 & 0 / 0\end{array}$

$\begin{array}{llll}388 & 0,86 & 0,67\end{array}$

$\begin{array}{llll}389 & 0,80 & 0,67\end{array}$

$\begin{array}{lll}390 & 1,00 & 1,00\end{array}$

$\begin{array}{lll}391 & 0,83 & 0,00\end{array}$

$\begin{array}{llll}392 & 0,93 & 0,71\end{array}$

$\begin{array}{lll}393 & 0,88 & 0,67\end{array}$

$\begin{array}{lll}394 & 1,00 & 1,00\end{array}$

$\begin{array}{lll}395 & 0,42 & 0,63\end{array}$

$\begin{array}{lll}396 & 0,64 & 0,47\end{array}$

$\begin{array}{llll}397 & 0,83 & 0,60\end{array}$

$\begin{array}{lll}398 & 1,00 & 1,00\end{array}$

$399 \quad 1,00 \quad 1,00$

$\begin{array}{lll}400 & 1,00 & 0 / 0\end{array}$

$401 \quad 0,63 \quad 0,76$

$\begin{array}{llll}402 & 0,88 & 0,83\end{array}$

$\begin{array}{lll}403 & 0,83 & 0,92\end{array}$

$404 \quad 0,31 \quad 0,50$

$\begin{array}{lll}405 & 1,00 & 0 / 0\end{array}$

$\begin{array}{llll}406 & 0,80 & 0,90\end{array}$

$\begin{array}{lll}407 & 0,67 & 0,50\end{array}$

$\begin{array}{llll}408 & 0,67 & 0,86\end{array}$

$\begin{array}{lll}409 & 0,29 & 0,55\end{array}$

$\begin{array}{lll}410 & 0,13 & 0,42\end{array}$

$\begin{array}{llll}411 & 0,25 & 0,73\end{array}$

$412 \quad 0,25 \quad 0,00$

$\begin{array}{llll}413 & 0,50 & 0,80\end{array}$

$\begin{array}{lll}414 & 0,33 & 0,00\end{array}$

$\begin{array}{llll}415 & 1,00 & 1,00\end{array}$

$416 \quad 1,00 \quad 1,00$

$\begin{array}{lll}417 & 1,00 & 0 / 0\end{array}$

$418 \quad 0,40 \quad 0,81$

$419 \quad 1,00 \quad 1,00$

$\begin{array}{lll}420 & 0,75 & 0,67\end{array}$

$421 \quad 1,00 \quad 1,00$

$422 \quad 1,00 \quad 1,00$

$4231,00 \quad 1,00$

$424 \quad 1,00 \quad 1,00$

$425 \quad 0,50 \quad 0,50$

$\begin{array}{llll}426 & 1,00 & 1,00\end{array}$

$427 \quad 1,00 \quad 1,00$

$\begin{array}{lll}428 & 1,00 & 0 / 0\end{array}$

$429 \quad 0,50 \quad 0,00$

$\begin{array}{lll}430 & 1,00 \quad 0 / 0\end{array}$

$\begin{array}{lll}431 & 0,50 & 0,00\end{array}$

$432 \quad 1,00 \quad 0 / 0$

$\begin{array}{lll}433 & 0,67 & 0,88\end{array}$

$\begin{array}{lll}434 & 0,33 & 0,43\end{array}$

$\begin{array}{lll}435 & 1,00 & 0 / 0\end{array}$

$\begin{array}{llll}436 & 0,33 & 0,82\end{array}$

$\begin{array}{llll}437 & 0,14 & 0,14\end{array}$

$\begin{array}{lll}438 & 0,29 & 0,55\end{array}$

$\begin{array}{llll}439 & 0,36 & 0,48\end{array}$

$\begin{array}{lll}440 & 0,26 & 0,45\end{array}$

$\begin{array}{llll}441 & 0,24 & 0,54\end{array}$

$\begin{array}{llll}442 & 0,21 & 0,42\end{array}$

$\begin{array}{lll}443 & 0,34 & 0,15\end{array}$

$\begin{array}{llll}444 & 0,23 & 0,66\end{array}$

$\begin{array}{llll}445 & 0,50 & 0,75\end{array}$

\begin{tabular}{lll}
446 & 0,67 & 0,80 \\
\hline
\end{tabular}

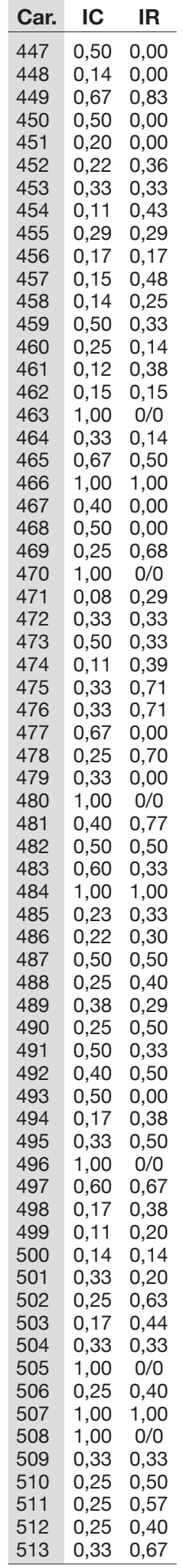

Car. IC IR

$\begin{array}{lll}4,20 & 0,00\end{array}$

$0,220,36$

$\begin{array}{lll}454 & 0,11 & 0,43\end{array}$

$\begin{array}{lll}455 & 0,29 & 0,29\end{array}$

$\begin{array}{ll}0,17 & 0,17\end{array}$

$\begin{array}{lll}458 & 0,14 & 0,25\end{array}$

$0,50 \quad 0,33$

$\begin{array}{lll}661 & 0,12 & 0,38\end{array}$

$0,15 \quad 0,15$

$\begin{array}{lll}4,04 & 0,33 & 0,14\end{array}$

$\begin{array}{lll}465 & 0,67 & 0,50\end{array}$

$\begin{array}{lll}1,00 & 1,00\end{array}$

$\begin{array}{lll}68 & 0,50 & 0,00\end{array}$

$0,25 \quad 0,68$

$\begin{array}{lll}471 & 0,08 & 0,29\end{array}$

$\begin{array}{ll}0,33 & 0,33\end{array}$

\begin{tabular}{l|ll}
\hline 74 & 0,11 & 0,39
\end{tabular}

$\begin{array}{lll}475 & 0,33 & 0,71\end{array}$

$\begin{array}{lll}0,33 & 0,71\end{array}$

$\begin{array}{lll}478 & 0,25 & 0,70\end{array}$

$\begin{array}{ll}0,33 & 0,00\end{array}$

$\begin{array}{lll}481 & 0,40 & 0,77\end{array}$

$0,50 \quad 0,50$

\begin{tabular}{lll}
483 & 0,60 & 0,33 \\
\hline 84 & 1,00 & 1,00
\end{tabular}

$\begin{array}{lll}8,85 & 0,23 & 0,33\end{array}$

$\begin{array}{lll}0,22 & 0,30\end{array}$

$\begin{array}{lll}488 & 0,25 & 0,40\end{array}$

$\begin{array}{ll}0,38 & 0,29\end{array}$

$\begin{array}{lll}91 & 0,50 & 0,33\end{array}$

$0,40 \quad 0,50$

\begin{tabular}{lll}
\hline 94 & 0,50 & 0,00 \\
\hline & 0,17 & 0,38
\end{tabular}

$\begin{array}{lll}495 & 0,33 & 0,50\end{array}$

$\begin{array}{lll}1,00 & 0 / 0\end{array}$

$\begin{array}{lll}988 & 0,17 & 0,38\end{array}$

$\begin{array}{lll}0,11 & 0,20\end{array}$

$\begin{array}{lll}0,14 & 0,14\end{array}$

$\begin{array}{llll}502 & 0,25 & 0,63\end{array}$

$\begin{array}{lll}0,17 & 0,44\end{array}$

$\begin{array}{lll}505 & 1,00 & 0 / 0\end{array}$

$\begin{array}{lll}5,025 & 0,40\end{array}$

$\begin{array}{lll}507 & 1,00 & 1,00\end{array}$

$\begin{array}{lll}0,25 & 0,50\end{array}$ 


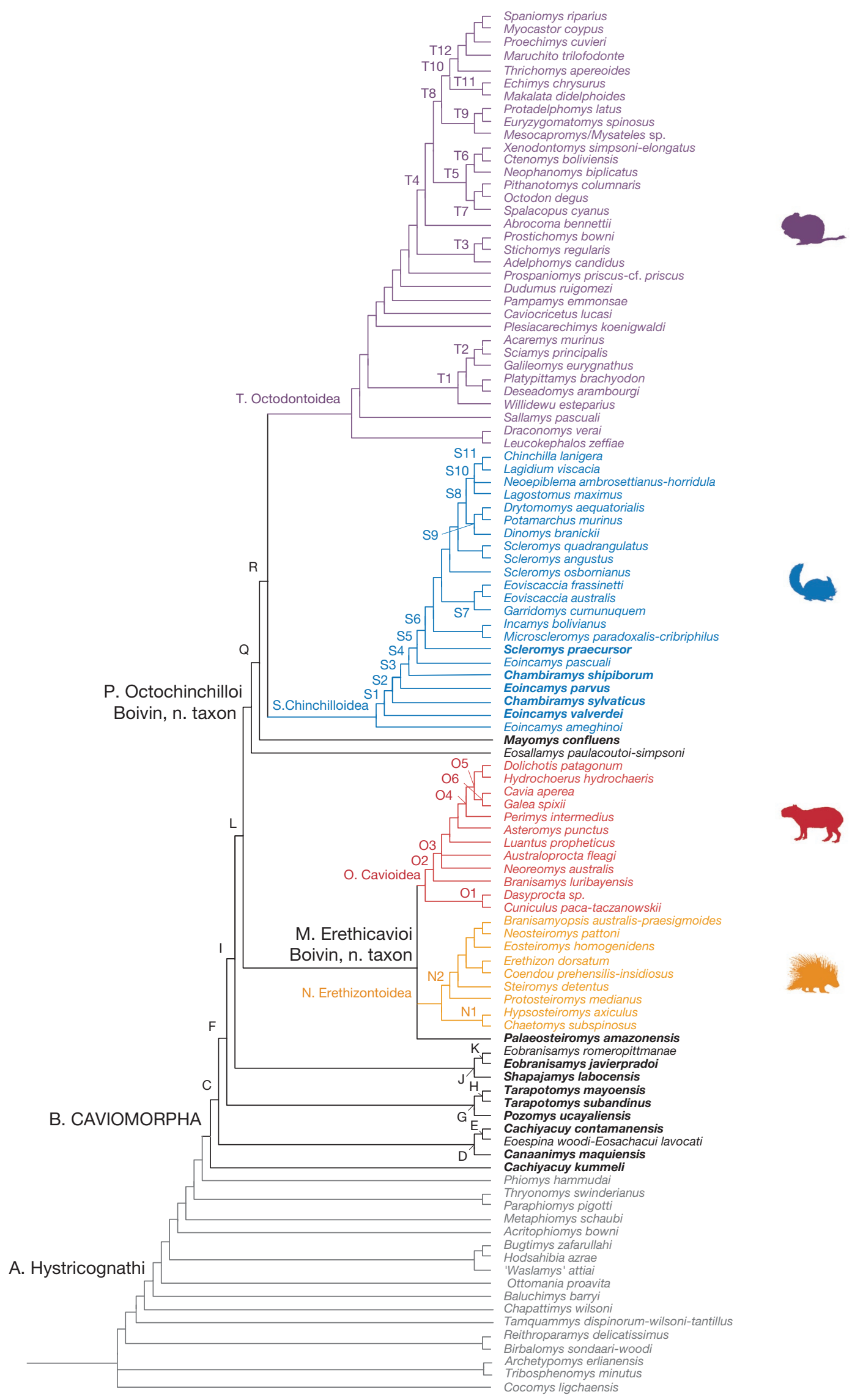

FiG. 5. - Résultats de l'AG1. Consensus strict de huit arbres équiparcimonieux de 3302,65 pas chacun (IC $=0,573 ; \mathrm{IR}=0,599)$. Les taxons en gras sont les espèces découvertes dans le Paléogène de Contamana (Éocène et Oligocène) et à Tarapoto/Shapaja. Le code couleur est le même que celui de la Figure 3. 


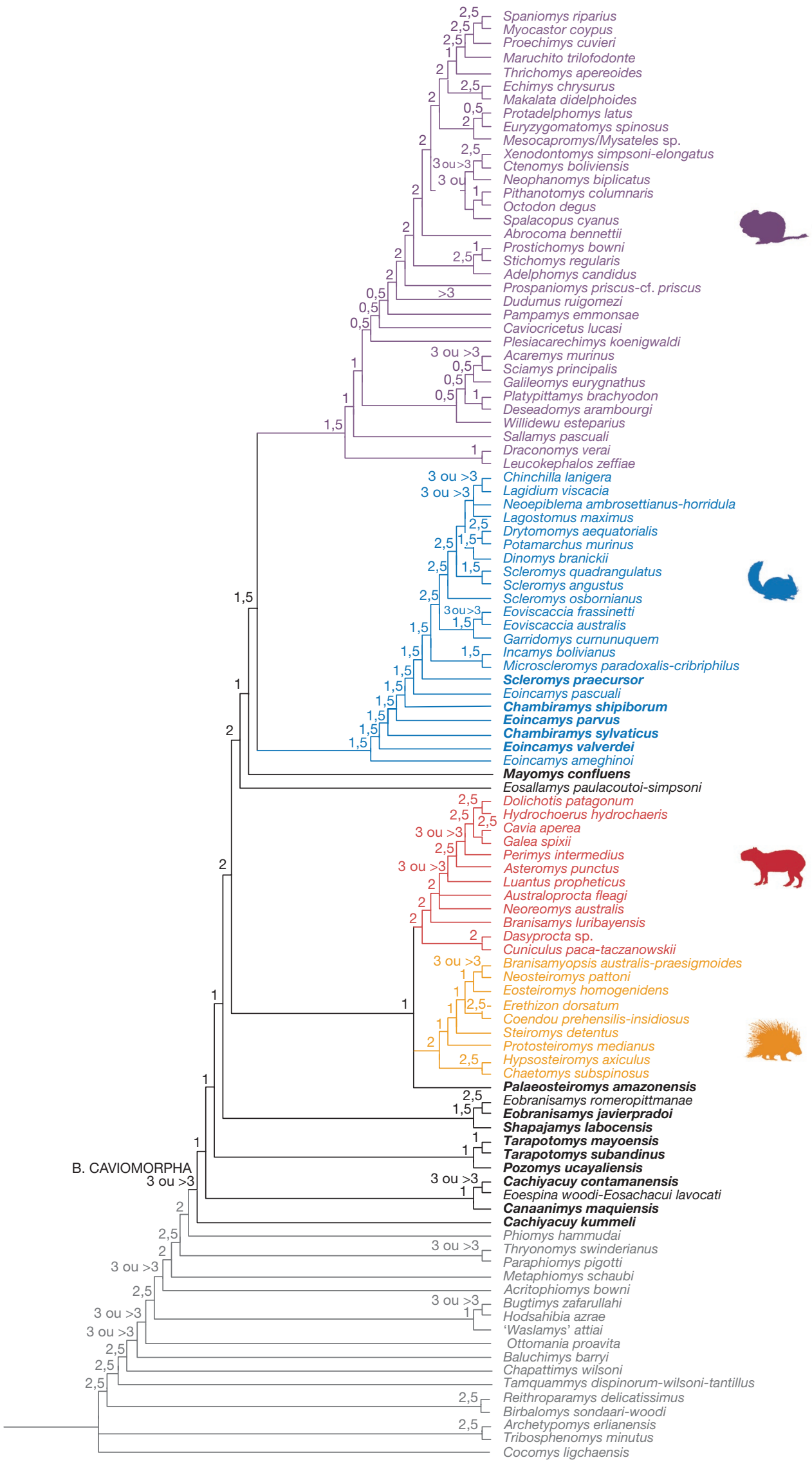

FIG. 6. - Consensus strict de l'AG1 (Fig. 5) et indices de Bremer aux noeuds. Les taxons en gras sont les espèces découvertes dans le Paléogène de Contamana (Éocène et Oligocène) et à Tarapoto/Shapaja. Le code couleur est le même que celui de la Figure 3. 
Noud F ( 5 NA et 6 A). Ce noud correspond à l'ensemble des autres caviomorphes, Cachiyacuy kummeli et le clade défini au nœud D en étant exclus. Parmi les cinq NA de ce clade, qui montrent tous des changements secondaires (réversions et/ ou convergences) par rapport au groupe de branchement, à Canaanimys, Eoespina et aux deux espèces de Cachiyacuy, notons: un alignement prononcé du bras antérieur de l'hypocône avec la mure sur les P4 (car. 621); la longueur des dP4 excédant leur largeur (car. 732); et la longueur des M1 supérieure ou égale à leur largeur (car. 1221).

Nœud G (3 NA et 12 A). Ce nœud correspond à l'un des clades issus du nœud F. Ce clade est composé de Pozomys, Tarapotomys mayoensis et T. subandinus. Il est soutenu par trois NA non codés pour T. subandinus (dont deux peu codés sur le reste de la matrice): un bras antérieur du métacône fin sur les molaires supérieures (car. 2050, caractère codé chez 33 taxons); un ectolophide aligné à l'hypolophide (car. $289^{1}$, caractère codé chez 52 taxons) et un bras antérieur de l'hypoconide peu élevé sur les p4 (car. 2910, caractère codé chez 29 taxons).

Nœud H (2 NA et 7 A). Ce nœud révèle la monophylie du genre Tarapotomys, dont les deux espèces partagent: des dents supérieures mésodontes (car. 91) et des M2 avec une longueur supérieure ou égale à leur largeur (car. 1231).

Noud I (3 NA et 5 A). Ce nœud correspond à l'ensemble des caviomorphes à l'exception de C. kummeli et des clades des nœuds $\mathrm{D}$ et $\mathrm{G}$. Les trois NA sont des réversions secondaires lorsqu'est considéré l'ensemble des taxons du nœud I. Cependant, deux semblent témoigner d'un signal phylogénétique relativement fort (voir Tableau 3) : la présence d'un mésolophe sur les P4 (car. 561; état partagé également avec la majorité des cavioïdes, éréthizontoïdes et chinchilloïdes codés; IC $=0,75$ et IR $=0,82$ ), et un métalophe connecté au postérolophe sur les M3 (car. 2174; même état chez la majorité des cavioïdes et éréthizontoïdes codés; $\mathrm{IC}=0,70$ et $\mathrm{IR}=0,70$ ). Le troisième NA (car. 2141) est la présence d'un éperon sur le postérolophe. Malgré de relativement bons indices IC $(0,85)$ et IR $(0,67)$, ce caractère est peu codé chez les éréthizontoïdes et apparait très polymorphe, particulièrement chez Phiomys hammudai et les caviomorphes basaux: C. kummeli, C. contamanensis, Eoespina, T. mayoensis, Shapajamys et Eobranisamys romeropittmanae.

Nœud J (3 NA et 8 A). Ce nœud correspond au clade issu du noud I et réunissant Shapajamys, Eobranisamys romeropittmanae et $E$. javierpradoi. Il est soutenu par trois NA: un protolophe labial transverse labialement et oblique lingualement (car. $84^{2}$, non codé chez E. romeropittmanae); la présence d'une néocristide 2 sur les dp4 (car. 3201, état présent seulement chez Shapajamys, E. romeropittmanae, Branisamys et Luantus); et un bras postérieur du protoconide long sur les m1-2 (car. 3720). Ce dernier caractère est très polymorphe au sein du groupe de branchement et des caviomorphes (E. romeropittmanae inclus).
Noud K (4 NA et 9 A). Ce nœud soutient la monophylie du genre Eobranisamys, dont les deux espèces ont notamment un métacône réduit sur les M3 (car. 2021) et ne présentent pas de protolophe lingual sur les M1-2 (car. 1471), ni sur les M3 (car. 151').

Noud $\mathrm{L}$ - le clade du groupe couronne des caviomorphes (4 NA et $8 \mathrm{~A}$ ). Ce clade exclut l'extrême majorité des taxons définis dans les localités éocènes de Contamana, à Santa Rosa et à Tarapoto/Shapaja (à l'exception de Palaeosteiromys, d'Eoincamys, de Chambiramys, d'Eosallamys et de Mayomys). Il regroupe les quatre super-familles des caviomorphes (Erethizontoidea, Cavioidea, Chinchilloidea et Octodontoidea) et les taxons fossiles qui leur sont étroitement apparentés. Les NA le soutenant sont relativement peu codés et/ou fortement homoplasiques (voir Tableau 3) : absence de mésolophule sur les $\mathrm{P} 4$ (car. $\left.52^{0} ; \mathrm{IC}=0,64 ; \mathrm{IR}=0,50\right)$; alignement prononcé du bras antérieur de l'hypocône avec la mure sur les M2 (car. $1971 ; \mathrm{IC}=0,44 ; \mathrm{IR}=0,55)$; un bras postérieur du protoconide court sur les $\mathrm{m} 3$ (car. $375^{1}$; IC $=0,35$; IR $=0,45$ ); et une crête massétérique modérément développée (car. 4411; $\mathrm{IC}=0,24 ; \mathrm{IR}=0,54)$.

Noud M - le clade des Erethicavioi Boivin, n. taxon (12 NA et 16 A; voir section «Systématique»). Ce nœud est bien soutenu (i.e., supporté par $12 \mathrm{NA}$ ) et correspond à une multifurcation. Il correspond au clade (Erethizontoidea, Cavioidea, Palaeosteiromys). Cette trifurcation est notamment soutenue par: une courte excroissance du protocône sur la P4 (car. 311); une longueur de la M2 similaire à sa largeur ou plus longue (car. 1231); l'absence du bras postérieur du paracône sur les molaires supérieures (car. 1421); les dp4 possédant un trigonide large (car. 3031); un long néomésolophide sur les molaires inférieures (car. 3841); et un entoconide aligné ou postérieur à l'hypocône sur les m2 (car. 3950). Le caractère 142 (peu codé chez les cavioïdes; $3 / 12$ taxons) est très polymorphe chez les éréthizontoïdes du nœud N2.

Nœud N - le clade des Erethizontoidea (4 NA et 15 A). Ce clade rassemble les taxons modernes d'éréthizontoïdes et l'ensemble des espèces fossiles qui leur sont apparentées. Il se caractérise par une dichotomie basale formant les nœuds $\mathrm{N} 1$ et N2. Il est soutenu par: l'absence du bras postérieur du protoconide sur les p4 (car. 2691); l'absence de la cristide/ éperon du métaconide sur les dp4 (car. 3170); un long bras postérieur du protoconide sur les $\mathrm{m} 3$ (car. 3750); et la présence sous la m1 d'une échancrure où s'insère le tendon du masseter medialis pars infraorbitalis (car. 4401). Pour ce dernier caractère, Coendou et Erethizon montrent une échancrure plus postérieure (entre la $\mathrm{m} 1$ et la $\mathrm{m} 2$, état 2 ). Les caractères 317 , pour lequel une réversion est observée chez Branisamyopsis, et 375 sont peu codés (respectivement chez 4/9 et 3/9 taxons).

Noud N1 (5 NA et $23 \mathrm{~A}$ ). Ce nœud traduit la proximité phylogénétique de Chaetomys et d'Hypsosteiromys. Ces deux genres partagent: des cristides obliques sur les molaires inférieures (car. $5^{1}$ et 2); une connexion entre le métacône et le 
postérolophe absente sur les $\mathrm{P} 4$ (car. $65^{\circ}$; un caractère retrouvé aussi chez Branisamyopsis) ; un mésostyle très développé sur les M1-2 (car. $166^{2}$; un caractère qui se retrouve aussi chez Branisamyopsis et Neosteiromys) ; un postérolophe peu ou pas connecté à l'hypocône sur les M3 (car. $223^{\circ}$; un caractère retrouvé aussi chez Steiromys) ; et un hypolophide fortement oblique sur les molaires inférieures (car. 4011).

Noud N2 (2 NA et 9 A). Ce nœud rassemble les autres éréthizontoïdes actuels inclus dans l'analyse (Coendou et Erethizon), associés à Protosteiromys, Steiromys, Eosteiromys, Branisamyopsis et Neosteiromys. Il est soutenu par 2 NA: une absence d'alignement prononcé de la mure avec le bras antérieur de l'hypocône sur les M3 (car. 1970 ; réversion chez Erethizon et Branisamyopsis) et une connexion entre l'entoconide (ou son bras postérieur) et le postérolophide sur les m1-2 (car. 3971).

Nœud O - le clade des Cavioidea (10 NA et 15 A). Ce clade regroupe les taxons modernes de cavioïdes et l'ensemble des taxons fossiles qui leur sont apparentés. Il se caractérise par une dichotomie basale formant les nœuds $\mathrm{O} 1$ et $\mathrm{O} 2$. Il réunit des formes notamment caractérisées par une couronne dentaire haute (car. 91, 2 et 3); une apophyse condyloïde basse (car. 4491); et un foramen dans le canal lacrymal très développé (car. 4812).

Noud O1 (7 NA et 18 A). Ce nœud exprime la proximité phylogénétique de Dasyprocta avec Cuniculus. Il est soutenu majoritairement par un ensemble de caractères crâniens, peu codés toutefois sur l'ensemble de la matrice: un foramen mentonnier situé dorsalement (car. $437^{2}$; caractère codé chez 41 taxons); une projection antérieure du prémaxillaire très développée (car. $455^{2}$; caractère codé chez 14 taxons); un processus postorbitaire développé (car. $472^{1}$; caractère codé chez 21 taxons) ; un processus postorbitaire composé du frontal et du squamosal (car. $473^{1}$; caractère codé chez 21 taxons); et un processus paroccipital connecté à la bulle auditive (car. $503^{1}$; caractère codé chez 23 taxons).

Nœud O2 - le clade des Caviidae (5 NA et 12 A). Ce nœud rassemble les taxons modernes de caviidés et les espèces fossiles qui leur sont directement apparentées (Branisamys, Neoreomys, Australoprocta, Luantus, Asteromys et Perimys). Parmi les cinq NA soutenant ce nœud, notons: des loph(-id)es très épais (car. $3^{2}$ ); l'absence du bras postérieur du protoconide (car. 3741) et la présence d'un mésolophide (car. 3891) sur les m3. Bien codés sur l'ensemble de la matrice, ces deux derniers caractères sont, en revanche, peu codés chez les caviidés (chez seulement 3/10 taxons).

Noud O3 (6 NA et 14 A). Ce nœud correspond au clade divergeant successivement au clade des caviidés. Cette trifurcation réunit les taxons du nœud $\mathrm{O} 2$, à l'exception de Branisamys. La séquence de branchement est de la forme (Neoreomys, Australoprocta, (Luantus, (Asteromys, (Perimys, (caviidés modernes))))). Ces taxons ont notamment des rangées dentaires supérieures convergentes (car. 111) et une échancrure où s'insère le tendon du masseter medialis pars infraorbitalis située sous la m1 (car. 4401).
Nœuds O3 à O4. Plusieurs autres nœuds sont présents (Fig. 5) mais ils ne seront pas décrits ici.

Nœud O4 (5 NA et 5 A). Ce nœud témoigne de la proximité phylogénétique de Perimys avec les taxons actuels de caviidés (Dolichotis, Hydrochoerus, Cavia et Galea). Ces taxons se caractérisent par: la présence de cément (car. 81 et 2 ; sauf Galea); une euhypsodontie (car. 93); et l'absence d'antéroflexide (car. 4091), de mésoflexide (car. 4111) et de métaflexide (car. 4131) sur des molaires inférieures modérément usées.

Nœud O5 - le clade du groupe couronne des caviidés (3 NA et $1 \mathrm{~A}$ ). Ce nœud rassemble les caviidés modernes (Dolichotis, Hydrochoerus, Cavia et Galea). Ils sont notamment caractérisés par: une projection antérieure sur la p4 (car. 2971) et une mandibule pseudo-sciurognathe (car. 4332).

Nœud O6 - le clade des caviinés (3 NA et 1 A). Ce nœud exprime la proximité phylogénétique de Cavia et Galea. Il est soutenu par trois caractères crâniens: un foramen lacrymal orienté latéralement (car. 4841); un maxillaire en contact avec l'alisphénoïde (car. $498^{\circ}$ ); et un sinus épitympanique complètement couvert par l'apophyse postérieure du squamosal (car. 5091).

Nœud P (3 NA et 13 A). Ce clade rassemble les taxons modernes de chinchilloïdes et d'octodontoïdes et les taxons fossiles qui leur sont directement apparentés, Eosallamys étant le groupe frère des autres taxons concernés. Ces taxons sont notamment caractérisés par: l'absence de métacône sur les P4 (car. 641' codés pour 11/33 taxons, les octodontoïdes retenant leurs dp4/ dP4 à l'âge adulte exclus) et un métacône, lorsqu'il est présent, positionné plus lingualement au paracône sur les M2 (car. $203^{2}$; codé pour six des neuf taxons dotés d'un métacône).

Nœud Q (6 NA et 8 A). Ce noud correspond au clade divergeant successivement au groupe du nœud P, avec Mayomys en position de groupe frère des autres taxons. Parmi les 6 NA soutenant ce nœud, notons: une absence de métalophe sur les dP4 (car. $115^{0}$; peu codé chez les chinchilloïdes : 4/22) et M1-2 (car. 2111); une absence d'éperon lingual du postérolophe sur les dP4 (car. 1171'; peu codé chez les chinchilloïdes: 3/22); et une connexion du mésostylide avec le métaconide sur les dp4 (car. 3141). Les caractères 117, 211 et 315 montrent des réversions chez certains chinchilloïdes et/ou octodontoïdes.

Nœud R - le clade des Octochinchilloi Boivin, n. taxon (3 NA et $2 \mathrm{~A}$; voir section "Systématique»). Ce nœud soutient la monophylie de l'ensemble (Chinchilloidea [nœud S], Octodontoidea [nœud T]). Il réunit les mêmes taxons que le nœud $\mathrm{P}$ à l'exception d'Eosallamys et Mayomys. Il se caractérise par une dichotomie basale formant les nœuds $S$ et T. Il est soutenu par: des M1 plus larges que longues (car. 1220); un métacône, lorsqu'il est présent, crestiforme sur les M3 (car. 2021 ; codés pour cinq des huit taxons avec un métacône); et un bras antérieur de l'hypoconide bas sur les dp4 (car. 3471). Cependant, la faible fréquence d'observation du caractère 347 (codé chez seulement 5/56 taxons) limite la bonne compréhension de son évolution. 
Noud S - le clade des Chinchilloidea (6 NA et 24 A). Ce nœud rassemble les chinchilloïdes modernes et l'ensemble des taxons fossiles qui leur sont apparentés. Ils sont notamment caractérisés par: une connexion entre l'antérolophe et le paracône sur les P4 (car. 271); l'absence d'un mésolophule sur les M1-2 (car. 1770); une extrémité linguale libre du mésolophe (car. 1830); et la présence d'un néomésolophide sur les p4 (car. 2721). Les caractères 27, 183 et 272 montrent chacun une réversion secondaire au sein des chinchilloïdes.

Noud S1 (3 NA et 6 A). Ce nœud correspond au clade divergeant successivement au groupe du nœud $S$ et excluant Eoincamys ameghinoi. Ils sont caractérisés par: une haute couronne dentaire (car. 91, 2 et 3 ; sauf pour Eoincamys pascuali); un protolophe lingual absent sur les P4 (car. 361) et les M3 (car. 1511; sauf pour Eoviscaccia australis).

Noud S2 (5 NA et $3 \mathrm{~A}$ ). Ce noud correspond au clade divergeant successivement au groupe du nœud S1 et excluant Eoincamys valverdei. Les NA soutenant ce nœud sont: des longueurs de la M1 (car. 1221) et de la M2 (car. 1231) supérieures ou égales à leur largeur; un protolophe lingual absent sur les M1-2 (car. 1471); un mésolophe lingualement connecté au postérolophe sur les M1-2 (car. 1834); et un postéroflexus absent sur des M2 modérément usées (car. 2301). Le caractère 183 est variable chez deux taxons (sur les 14 taxons codés pour ce caractère; état 0 ) et hautement polymorphe chez quatre autres.

Nœud S3 (2 NA et 4 A). Ce nœud correspond au clade divergeant successivement au groupe du noud S2 et excluant Chambiramys sylvaticus. Il est soutenu par deux NA: des crêtes fortement obliques sur les molaires supérieures (car. $4^{2}$ ) et une absence du bras antérieur de l'hypoconide sur les molaires inférieures (car. 4031). Cependant, certains taxons issus de ce nœud (Incamys, Microscleromys, Chinchilla et Lagidium) montrent des crêtes des molaires supérieures moins obliques à transverses.

Noud S4 (2 NA et 4 A). Ce nœud correspond au clade divergeant successivement au groupe du noud S3 et excluant Eoincamys parvus. Il est soutenu par deux NA: l'absence de métacône sur les M1-2 (car. 1991) et l'absence de connexion entre le métalophulide I et le métaconide sur dp4 (car. 3110). Le premier NA montre une réversion chez les dinomyidés et le second caractère est très peu contrôlé (chez seulement 3/18 taxons).

Nœud S5 (1 NA et 6 A). Ce nœud correspond au clade divergeant successivement au groupe du nœud $\$ 4$ et excluant Chambiramys shipiborum. Il est soutenu par un NA peu codé (chez seulement 5/17 taxons) correspondant à l'absence du bras antérieur de l'entoconide sur les molaires inférieures (car. 3960).

Nœud S6 (2 NA et 10 A). Ce nœud correspond au clade divergeant successivement au groupe du nœud $\mathrm{S} 5$. Il réunit les mêmes taxons que le noud S5 à l'exception d'Eoincamys pascuali. Les taxons de ce clade sont caractérisés par: des loph(-id)es très épais (car. $3^{2}$ ) et une couronne dentaire haute (car. 92 et 3).
Noud S7 (4 NA et 6 A). Ce nœud témoigne de la proximité phylogénétique de Garridomys avec les deux espèces d'Eoviscaccia (E. frassinettii et $E$. australis). Ces taxons se caractérisent notamment par: un mésolophe lingualement connecté au postérolophe sur les P4 (car. 572) et la formation de lobes sur les molaires supérieures (car. 2351) et inférieures (car. 4181).

Noud S8 - le clade du groupe couronne des chinchilloïdes $(8$ NA et 16 A). Ce nœud rassemble les chinchilloïdes modernes (Chinchilla, Lagidium, Lagostomus et Dinomys) et les taxons fossiles qui leur sont directement apparentés (Neoepiblema, Drytomomys et Potamarchus). Ils sont notamment caractérisés par: une importante hétérogénéité de l'épaisseur de l'émail sur les molaires (car. 141; sauf pour Neoepiblema); la présence de lamines sur les M1-2 (car. 2441), les M3 (car. 2451) et sur les molaires inférieures (car. 4261); un diastème inférieur court (car. 4341); et la présence sous la m1 d'une échancrure où s'insère le tendon du masseter medialis pars infraorbitalis (car. $440^{1}$; sauf chez Neoepiblema où l'échancrure est située entre la $\mathrm{m} 1$ et la m2).

Noud S9 - le clade des Dinomyidae sensu stricto (4 NA et 10 A). Ce nœud regroupe Dinomys et les taxons fossiles qui lui sont directement apparentés (Drytomomys et Potamarchus). Ces taxons sont notamment caractérisés par: la présence d'un métalophe sur les M1-2 (car. 2110); la présence d'un antéroflexide sur les molaires inférieures (car. 4090); et un diastème supérieur court (car. 4612).

Noud S10 (9 NA et 8 A). Cette trifurcation très soutenue (i.e., supportée par 9 NA et indice de Bremer égal ou supérieur à 3) rassemble les chinchillidés modernes (Chinchilla, Lagidium et Lagostomus) et le taxon fossile Neoepiblema. Les NA la soutenant sont: l'absence d'un alignement du bras antérieur de l'hypocône avec la mure et avec le protolophe labial sur les P4 (car. $62^{\circ}$ et $63^{\circ}$ ) et sur les molaires supérieures (car. 1960 $197^{\circ}$ et $198^{\circ}$ ); trois lamines sur les M1-2 (2480); la présence d'un bras postérieur du métaconide sur les $\mathrm{p} 4$ (car. 2630); l'absence d'un néomésolophide sur les molaires inférieures (car. 3830); et un foramen dans le canal lacrymal de taille moyenne (car. 4811). Lagostomus n'est codé pour aucun de ces NA, excepté le caractère 481.

Noud S11 - le clade des Chinchillinae (9 NA et 2 A). Ce nœud exprime la proximité phylogénétique de Chinchilla avec Lagidium. Il est soutenu essentiellement par des caractères crâniens et par quelques caractères dentaires: des lamines légèrement obliques sur les molaires supérieures (car. 41); une P4 plus grande que la M1 (car. 220); un bras antérieur de l'hypocône longitudinal sur la P4 (car. 612); une échancrure où s'insère le tendon du masseter medialis pars infraorbitalis connectée à la crête latérale (car. 4393); un long foramen incisif (car. 4570); des marges antérieures des choanes subperpendiculaires à l'axe antéropostérieur du crâne (car. 4921); un processus paroccipital orienté ventralement ou ventrolatéralement (car. 5020) et entièrement lié à la bulle auditive (car. 5031). 
Noud T - le clade des Octodontoidea (8 NA et 27 A). Ce nœud soutenu par $8 \mathrm{NA}$ rassemble les octodontoïdes modernes et les taxons fossiles qui leur sont directement apparentés. La séquence de branchement est de la forme ((Draconomys, Leucokephalos), (Sallamys, (nœud T1, autres octodontoïdes))). Les membres de ce groupe montrent: un bras antérieur du métaconide dépourvu de connexion labiale (libre) sur les p 4 (car. 2620); l'absence du bras postérieur du métaconide (car. 2631) et du mésostylide (car. 2650) sur les p4; un court bras postérieur du métaconide sur les molaires inférieures (car. $360^{1}$ ); l'absence de mésostylide (car. 3660), de néomésolophide (car. 3830) et du bras antérieur de l'entoconide (car. 3960) sur les molaires inférieures; et une réduction importante de l'antéroflexide sur les molaires inférieures (car. 4101).

Noud T1 - le clade de la famille éteinte des Acaremyidae (2 NA et $9 \mathrm{~A})$. Ce nœud témoigne de la proximité phylogénétique entre six taxons éteints: Acaremys, Sciamys, Galileomys, Platypittamys, Deseadomys et Willidewu. Il est soutenu par deux NA: l'absence sur les M3 d'un alignement prononcé de la mure avec le bras antérieur de l'hypocône (car. 1970) et la présence d'un éperon du métaconide sur les molaires inférieures (car. 36911). Le caractère 197 montre, cependant, une réversion chez Acaremys et Sciamys (car. 1971), et le caractère 369 est polymorphe chez Platypittamys, Galileomys et Sciamys $\left(369^{0+1}\right)$.

Noud T2 $(5$ NA et 6 A). Ce nœud révèle la proximité phylogénétique entre trois taxons éteints: Acaremys, Sciamys et Galileomys, ce dernier étant le premier à diverger. Ces trois taxons se caractérisent par: une courte excroissance du protocône (car. 310), la présence d'un mésostyle (car. 311) et d'une troisième crête transverse (car. 511) sur les $\mathrm{P} 4$; une tendance à développer une connexion du bras postérieur du protoconide avec l'éperon du métaconide sur les $\mathrm{p} 4$ (car. 27033 un caractère se retrouvant également chez Sallamys et Platypittamys) et sur les m3 (car. 3764).

Nouds T1 à T3. Plusieurs autres nœuds sont présents (Fig. 5) mais ils ne seront pas décrits ici. La séquence de branchement est de la forme (nœud T1, (Plesiacarechimys, (Caviocricetus, (Pampamys, (Dudumus, (Prospaniomys, (nœudT3, (nœud T4)))))))).

Noud T3 - le clade des Adelphomyidae Patterson \& Pascual, 1968 , n. stat. (4 NA et 7 A). Ce nœud rassemble trois octodontoïdes fossiles, Prostichomys, Stichomys et Adelphomys, ce dernier étant le premier à diverger. Ce nœud est soutenu par: une extension moyenne de l'hypoflexus sur des M1-2 modérément usées (car. 23411); une connexion du bras postérieur du protoconide avec le néomésolophide ou le mésolophide sur les dp4 (car. 3232); un alignement de l'hypolophide avec l'ectolophide sur les molaires inférieures (car. 4021); et la crête massétérique sur le dentaire modérément développée (car. 4411).

Nœud T4 - le clade du groupe couronne des octodontoïdes (4 NA et 7A). Ce nœud réunit les octodontoïdes modernes et les taxons fossiles qui leur sont directement apparentés. Ils se caractérisent notamment par: des dents à couronne haute (car.
91, 2 et 3); une absence du bras postérieur du protoconide sur les molaires inférieures (car. 3711); une absence de foramen mentonnier (car. 436'1).

Noud T5 (7 NA et $14 \mathrm{~A}$ ). Ce nœud très soutenu (i.e., supporté par 7 NA et indice de Bremer égal ou supérieur à 3) correspond au clade (Ctenomyidae, Octodontidae). Il est notamment soutenu par: une absence d'antéroflexide (car. 3491) et de métaflexide (car. 3511) sur des dp4 modérément usées; une m2 plus longue que la m3 (car. $354^{2}$; sauf pour Xenodontomys); une absence de mésoflexide sur des molaires inférieures modérément usées (car. 4111); et la formation avec l'usure de lobe sur les molaires inférieures (car. 4181).

Noud T6 - le clade des Ctenomyidae (3 NA et 14 A). Ce nœud regroupe Ctenomys et les taxons fossiles qui lui sont directement apparentés (Neophanomys et Xenodontomys). Il est soutenu par: une absence de mésoflexide sur les $\mathrm{dp} 4$ (car. 3501) et de métaflexide sur les molaires inférieures (car. 4131) modérément usées; et une fusion complète avec l'usure des lophides sur les molaires inférieures (car. 4231).

Nœud T7 - le clade des Octodontidae (7 NA et 2 A). Ce nœud soutenu par 7 NA réunit les octodontidés modernes inclus dans cette analyse (Octodon et Spalacopus) et un taxon fossile qui leur est directement apparenté (Pithanotomys). Parmi les sept NA de ce nœud, notons: des dents euhypsodontes (car. 93); une absence de paraflexus sur des molaires supérieures modérément usées (car. 2261) ; la forme ovale des lobes antérieurs (car. 4203) et postérieurs (car. 4213) sur les m1-2; et une fusion entre le métalophulide I et la deuxième cristide sur les m1-2 (car. 4241), ces deux structures ne fusionnant pas avec l'hypolophide (car. 4250).

Noud T8 - le clade des Echimyidae (3 NA et 2 A). Ce noud rassemble les échimyidés modernes inclus dans cette analyse et les taxons fossiles qui leurs sont directement apparentés. Ils montrent entre autres un entoconide situé plus mésialement à l'hypoconide sur les dp4 (car. 3402); et un fort développement de la projection antérieure du prémaxillaire (car. 4511).

Noud T9 (3 NA et 11 A). Ce nœud exprime la proximité phylogénétique entre Mesocapromys/Mysasteles, Euryzygomatomys et le taxon fossile Protadelphomys. Mesocapromys/Mysasteles est le premier des trois à diverger. Il est soutenu par: un alignement prononcé du bras antérieur de l'hypocône avec la mure sur les M1-2 (car. 1961) et sur les M3 (car. 1971), et une tendance à l'alignement du protolophe labial avec le bras antérieur de l'hypocône-mure sur les M1-2 (car. 198¹).

Noud T10 - le clade des Echimyinae (2 NA et 11 A). Ce nœud rassemble les échimyinés modernes inclus dans cette analyse et les taxons fossiles qui leur sont directement apparentés. Il se caractérise par une dichotomie basale formant les nœuds $\mathrm{T} 11$ et T12. Les deux NA soutenant ce clade sont: la présence d'une troisième cristide sur les dp4 (car. 3271) et d'un foramen palatin peu développé ou absent sur le crâne (car. 4881). 
Noud T11 - le clade des Echimyini (10 NA et 5 A). Ce nœud témoigne de la proximité phylogénétique entre Echimys et Makalata. Parmi les dix NA de ce nœud, notons: quatre racines sur les molaires supérieures (car. 151); la présence de lamines sur les M1-2 (car. 2441) et sur les M3 (car. 2451); l'absence de l'ectolophide distal sur les dp4 (car. 3370); un foramen incisif de faible diamètre (car. 4572); un court diastème supérieur (car. 4612); et la présence d'un foramen ethmoïdal entre la M1 et la M2 (car. 4850).

Noud T12 - le clade des Myocastorini (1 NA et 7 A). Ce nœud rassemble les Myocastorini modernes inclus dans cette analyse (Myocastor, Proechimys et Thrichomys) et les taxons fossiles qui leur sont directement apparentés (Spaniomys et Maruchito). Ce noud n'est soutenu que par un seul NA: un foramen lacrymal s'ouvrant au niveau du maxillaire (car. 4831). Spaniomys montre une réversion pour ce caractère, son foramen lacrymal s'ouvrant au niveau de la portion orbitale du lacrymal (car. 4830).

\section{AG1 non équilibrée}

Les analyses heuristiques effectuées sur le jeu de données taxons/caractères de l'AG1 non équilibrée ont généré 872 arbres équiparcimonieux de 3839 pas (IC =0,547; IR =0,593). L'arbre de consensus strict est présenté en Annexe 4.

Le consensus strict de l'AG1 non équilibrée montre de fortes irrésolutions qui concernent de nombreux taxons. Les relations entre super-familles sont irrésolues excepté l'association éréthizontoïdes + cavioïdes. En outre, les clades soutenus ont des topologies généralement différentes de celles de l'AG1 au niveau de la position des taxons fossiles.

AUTRES ANALYSES CONTRAINTES (DE L'AG2 A L'AG9) $A G 2$ : inclusion de Gaudeamus aslius, G. hylaeus et G. aegyptius

Les analyses heuristiques effectuées sur le jeu de données taxons/caractères de l'AG2 ont généré 42 arbres équiparcimonieux de 3493,48 pas ( $\mathrm{IC}=0,582 ; \mathrm{IR}=0,597)$. L'arbre de consensus strict est présenté en Annexe 4.

Gaudeamus aslius, G. hylaeus et G. aegyptius divergent au sein du groupe interne parmi les chinchilloïdes. Leur inclusion engendre plusieurs modifications des relations de parenté par rapport à l'AG1 : la divergence de Mayomys à la base des octodontoïdes; le branchement de Protadelphomys vers la base des chinchillö̈des qui, avec les trois espèces de Gaudeamus, forment alors un clade; et des branchements différents pour plusieurs chinchilloïdes (en groupe basal ou groupe couronne). Gaudeamus se caractérise par des loph(-id)es hauts et une tendance à l'hypsodontie, des traits retrouvés chez les caviomorphes. En outre, le patron dentaire de Gaudeamus montre plusieurs traits typiques (mais probablement convergents ?) des chinchilloïdes (i.e., taeniodontie, l'obliquité des loph(id)es, un métacône et un métalophe qui disparaissent et un mésolophe lingualement libre ou connecté au postérolophe sur ses molaires supérieures, et une deuxième cristide réduite sur les molaires inférieures), expliquant la position de Gaudeamus au sein de cette super-famille.
Remarque. À l'instar du résultat obtenu en AG2, plusieurs études récentes effectuées sans contrainte topologique soutiennent une affiliation de l'hystricognathe africain Gaudeamus aux caviomorphes (Sallam et al. 2009, 2011; Coster et al. 2010, 2012; Antoine et al. 2012). L'hypothèse la plus parcimonieuse induite par cette relation de parenté impliquerait notamment un scénario de dispersion transatlantique de rongeurs caviomorphes de l'Amérique du Sud vers l'Afrique, un sens de dispersion peu envisageable si l'on tient compte des contraintes paléobiogéographiques et environnementales (voir ci-dessous). Cette dispersion serait antérieure à $34 \mathrm{Ma}$, âge des plus anciens dépôts à Gaudeamus, et aurait probablement eu lieu à l'Éocène supérieur. Actuellement, l'hypothèse la plus souvent évoquée pour expliquer l'origine des caviomorphes en Amérique du Sud suggère un seul évènement de dispersion transatlantique des rongeurs hystricognathes à l'Éocène moyen (antérieure à $41 \mathrm{Ma}$ ) depuis l'Afrique vers l'Amérique du Sud (voir Lavocat 1973; Huchon \& Douzery 2001; Antoine et al. 2012). Le contexte de cette dispersion à l'origine des caviomorphes (i.e., isolement de l'Amérique du Sud, absence de registre fossile de formes apparentées sur les autres continents et paléocourants atlantiques orientés de l'Afrique vers Amérique du Sud; Houle 1998, 1999 ; Oliveira et al. 2009) rend pour l'instant improbable une dispersion des rongeurs hystricognathes entre l'Amérique du Sud et l'Afrique à l'Éocène supérieur (Coster et al. 2010, 2012; Sallam et al. 2011; Antoine et al. 2012). Une hypothèse alternative serait l'interprétation du patron dentaire de Gaudeamus comme résultant d'une évolution convergente entre les Gaudeamuridae africains et certains caviomorphes (Coster et al. 2010, 2012; Sallam et al. 2011; Antoine et al. 2012). Si les espèces attribuées au genre Gaudeamus sont essentiellement connues par des restes dentaires, quelques crânes et mandibules ont toutefois été décrits, notamment pour G. aslius (Wood 1968; Sallam et al. 2011). Cependant, ces derniers étant très déformés et fragmentaires (Sallam et al. 2011), ils ne permettent pas de statuer en faveur de l'une ou l'autre des hypothèses indépendamment de la signature dentaire. La question reste donc ouverte.

AG3: inclusion d' "Asteromys » bolivianus, de Cephalomys arcidens, Litodontomys et de Soriamys non contraints Les analyses heuristiques effectuées sur le jeu de données taxons/caractères de l'AG3 ont généré deux arbres équiparcimonieux de 3393,98 pas $(\mathrm{IC}=0,562 ; \mathrm{IR}=0,592)$. Larbre de consensus strict est présenté en Annexe 4.

"Asteromys» bolivianus, C. arcidens et Litodontomys forment un clade avec Asteromys punctus et se branchent au sein des chinchilloidés basaux. L'association d' 'Asteromys» bolivianus, de C. arcidens et de Litodontomys repose sur plusieurs NA observés sur des molaires inférieures modérément usées: l'absence de l'antéroflexide (car. 4091) et du métaflexide (car. 4131); et la fusion du métalophulide I avec seulement la deuxième cristide transverse (avec l'usure; car. $424^{1}$ et $425^{\circ}$ ). Le rapprochement d' 'Asteromys» bolivianus, C. arcidens, Litodontomys et A. punctus avec le clade (Chinchilloidea, Octodontoidea) et leur divergence au sein des chinchilloïdes sont notamment 
soutenues par: la présence d'un protolophe labial oblique sur les dP4 (car. 841) ; un mésostyle non isolé postérieurement sur les M1-2 (car. 17111); l'absence de mésolophule sur les M3 (car. 1780); et un métacône fortement connecté au postérolophe sur les M3 (car. 2091). A l'échelle du clade ( $A$. punctus, (Litodontomys, ("Asteromys» bolivianus, C. arcidens))), ces quatre NA sont codées uniquement chez "Asteromys» bolivianus et/ou C. arcidens. Soriamys se branche au sein des caviidés basaux, entre Luantus et Perimys. Son association avec Perimys et le groupe couronne des caviidés repose sur quatre NA: l'absence de protolophe labial (car. 330) et de mure (car. 390) sur les $\mathrm{P} 4$; une réduction du paraflexus importante sur des M2 modérément usées (car. 2272) ; et une extension transversale de l'hypoflexus importante sur des M1-2 modérément usées (car. 23422). L'inclusion de ces quatre taxons engendre d'importantes modifications des relations de parenté par rapport à celles obtenues à l'AG1: l'ensemble des caviomorphes basaux dans l'AG1 sont ici plus étroitement apparentés aux (octodontoïdes + chinchilloïdes); Draconomys est situé à la base des caviomorphes; Palaeosteiromys est associé à Dasyprocta et Cuniculus; Protadelphomys diverge au sein des chinchilloïdes; et plusieurs relations changent au sein des chinchilloïdes et des octodontoïdes.

AG4: inclusion d' "Asteromys» bolivianus, Asteromys punctus, de Cephalomys arcidens, Litodontomys et de Soriamys, tous contraints en un clade divergeant au sein des chinchilloïdes

Les analyses heuristiques effectuées sur le jeu de données taxons/ caractères de l'AG4 ont généré 291 arbres équiparcimonieux de 3397,47 pas (IC = 0,511; IR = 0,591). L'arbre de consensus strict est présenté en Annexe 4.

Les cinq taxons contraints forment le clade (Soriamys, (A. punctus, (Litodontomys, (C. arcidens, "Asteromys» bolivianus)))) situé à la base des chinchillö̈des. Cette contrainte génère d'importantes modifications des relations de parenté par rapport à l'AG1. Par exemple, Mayomys et Eosallamys divergent au sein des caviomorphes basaux de l'AG1. De fortes irrésolutions concernant les octodontoïdes basaux sont également observées.

AG5: inclusion d' "Asteromys » bolivianus, Asteromys punctus, de Cephalomys arcidens, Litodontomys et de Soriamys, tous contraints en un clade divergeant au sein des cavioides

Les analyses heuristiques effectuées sur le jeu de données taxons/caractères de l'AG5 ont généré six arbres équiparcimonieux de 3398,14 pas $(\mathrm{IC}=0,561 ; \mathrm{IR}=0,591)$. L'arbre de consensus strict est présenté en Annexe 4.

Les cinq taxons contraints forment avec Luantus le clade (Soriamys, Luantus, (A. punctus, (Litodontomys, (C. arcidens, "Asteromys" bolivianus))))) qui constitue le groupe-frère de (Perimys, ((Cavia, Galea), (Dolichotis, Hydrochoerus)). Cette contrainte génère d'importantes modifications des relations de parenté par rapport à l'AG1 au niveau notamment des groupes basaux de caviomorphes, des éréthizontoïdes et des cavioïdes (e.g. regroupement des taxons fossiles Australoprocta,
Branisamys, Neoreomys et Palaeosteiromys avec Cuniculus et Dasyprocta; association des deux espèces de Tarapotomys aux caviö̈des).

AG6: inclusion d' "Asteromys " bolivianus, de Cephalomys arcidens et de Litodontomys, non contraints

Les analyses heuristiques effectuées sur le jeu de données taxons/caractères de l'AG6 ont généré quatre arbres équiparcimonieux de 3372,97 pas (IC = 0,565; IR = 0,594). L'arbre de consensus strict est présenté en Annexe 4.

"Asteromys» bolivianus, C. arcidens et Litodontomys forment un clade qui se branche au sein des caviidés basaux, entre Asteromys et Perimys. L'association de ces trois taxons repose sur deux NA: la forme ovale du lobe antérieur (car. 4203) et du lobe postérieur (car. 4213) des m1-2. Leur divergence au sein des caviidés basaux est soutenue par deux NA: l'absence de l'antéroflexide (car. 4091) et du métaflexide (car. 4131) sur des molaires inférieures modérément usées. Leur inclusion engendre d'importantes modifications des relations de parenté par rapport à l'AG1: Cachiyacuy kummeli, Canaanimys et le clade (Shapajamys, (Eobranisamys romeropittmanae, E. javierpradoi)) sont plus étroitement apparentés aux octodontoïdes + chinchilloïdes; Eosallamys diverge à la base des cavioïdes et (Pozomys, Tarapotomys mayoensis) ainsi que T. subandinus à la base des caviidés; Australoprocta, Branisamys, Neoreomys et Palaeosteiromys sont plus étroitement apparentés à Cuniculus et Dasyprocta; et des changements de relations sont observés au sein des éréthizontoïdes.

AG7: inclusion d' 'Asteromys " bolivianus, Asteromys punctus, de Cephalomys arcidens et de Litodontomys, tous contraints en un clade divergeant dans les chinchilloïdes Les analyses heuristiques effectuées sur le jeu de données taxons/ caractères de l'AG7 ont généré 52 arbres équiparcimonieux de 3373,31 pas $(\mathrm{IC}=0,565$; IR =0,594). L'arbre de consensus strict est présenté en Annexe 4.

Les quatre taxons contraints forment le clade (A. punctus, (Litodontomys, (C. arcidens, "Asteromys» bolivianus))) qui diverge parmi les chinchilloïdes basaux, entre (Eoincamys ameghinoi, E. valverdei) et Protadelphomys. En plus d'une divergence de Protadelphomys au sein des chinchilloïdes, cette contrainte génère d'autres modifications des relations de parenté par rapport à l'AG1, notamment au niveau des groupes basaux de caviomorphes.

\section{AG8: inclusion de Plesiosteiromys}

Les analyses heuristiques effectuées sur le jeu de données taxons/caractères de l'AG8 ont généré 16 arbres équiparcimonieux de 3304,98 pas (IC = 0,573; IR =0,599). L'arbre de consensus strict est présenté en Annexe 4.

Plesiosteiromys se branche parmi les caviomorphes basaux, au niveau du nœud équivalent au nœud G de l'analyse AG1. Plesiosteiromys s'associe avec Tarapotomys subandinus. Les relations avec les autres membres du noud sont irrésolues: (Pozomys, T. mayoensis, (Plesiosteiromys, T. subandinus)). Ce nœud (équivalant du nœud G dans l'AG1) est soutenu par un seul NA: l'absence du protolophe lingual sur les P4 (car. 
361), mais ce caractère est seulement codé chez les deux espèces de Tarapotomys. L'association de Plesiosteiromys avec T. subandinus repose sur un NA peu codé pour les taxons du groupe interne (29/91) : un bras postérieur du paracône épais sur les molaires supérieures (car. 1431).

\section{AG9: inclusion de Loretomys}

Les analyses heuristiques effectuées sur le jeu de données taxons/caractères de l'AG9 ont généré 54 arbres équiparcimonieux de 3308,15 pas ( $\mathrm{IC}=0,573$; $\mathrm{IR}=0,599)$. L'arbre de consensus strict est présenté en Annexe 4.

Loretomys se branche au sein des octodontoïdes, au niveau du nœud équivalent au nœud T1 de l'analyse AG1. Ce nœud présente la même structure globale que dans l'AG1 et est soutenu par les mêmes NA (car. 1970 et 3691). L'inclusion de Loretomys engendre en revanche une irrésolution au niveau de son branchement avec Deseadomys et Platypittamys.

\section{DISCUSSION}

Les divers points discutés dans cette partie reposent essentiellement sur la topologie et la distribution des caractères obtenues dans l'AG1 (analyse principale contrainte pour laquelle les caractères ordonnés ont été équilibrés). Le consensus strict de cette analyse consitue la topologie de référence pour toutes les discussions qui vont suivre. Toutefois, certains points précis s'appuieront également sur les résultats issus des autres analyses contraintes (AG2-AG9).

\section{PHYLOGÉNIE ET IMPLICATIONS SYSTÉMATIQUES}

\section{DES CAVIOMORPHA}

Les groupes basaux des caviomorphes

L'incorporation de nombreux taxons incluant des représentants des quatre super-familles de caviomorphes dans une analyse cladistique (ici l'AG1) permet, pour la première fois, la mise en évidence de plusieurs groupes basaux au sein du clade Caviomorpha (en dehors du groupe apical). Ils sont au nombre de quatre (Cachiyacuy kummeli, nœud D, nœud G et nœud J) et concernent dix espèces fossiles. Sur ces dix, cinq proviennent des localités éocènes de Contamana que sont CTA27 (Cachiyacuy kummeli, Cachiyacuy contamanensis, Canaanimys maquiensis et Eobranisamys javierpradoi; Antoine et al. 2012; Boivin et al. 2017a) et CTA-29 (Pozomys ucayaliensis; Boivin et al. 2017a), et trois des localités de Tarapoto/Shapaja d'âge oligocène inférieur et dénommées TAR-01 (Shapajamys labocensis et Tarapotomys mayoensis; Boivin et al. 2018a) et TAR-21+TAR-22 (Tarapotomys subandinus; Boivin et al. 2018a). Les deux taxons restants, Eobranisamys romeropittmanae et Eoespina woodi-Eosachacui lavocati proviennent du site de Santa Rosa, une autre localité d'Amazonie péruvienne (?Éocène supérieur/Oligocène inférieur; Frailey \& Campbell 2004). Selon les résultats de l'AG7, Plesiosteiromys, découvert dans la localité CTA-61 (Oligocène supérieur, Contamana) et provisoirement attribué aux "Erethizontoidea ?" (Boivin et al. 2017b), diverge également précocement au sein des caviomorphes basaux.
Une position basale au sein des caviomorphes avait déjà été proposée pour trois d'entre eux: C. kummeli, C. contamanensis et $C$. maquiensis (Antoine et al. 2012; Boivin et al. $2017 \mathrm{a}, \mathrm{b}$ ). En effet, ces taxons présentent une suite de caractères apparaissant plésiomorphes au sein des caviomorphes et qui s'observent également chez plusieurs des plus anciens hystricognathes afro-asiatiques: (1) petite taille, (2) brachyodontie, (3) bunolophodontie, des loph(-id)es (4) fins et (5) transverses, (6) des molaires inférieures tétralophodontes, et des M1-2 (7) pentalophodontes avec (8) une connexion linguale entre la troisième crête transverse et le métalophe. La plésiomorphie de ces états a été vérifiée chez les caviomorphes dans l'AG1 pour le:

- (2) avec le car. $9^{0}$;

- (4) avec le car. 31 ;

- (5) avec les car. $4^{0}$ (pour dents supérieures) et $5^{0}$ (pour les dents inférieures);

- (6) avec les car. $370^{\circ}$ (absence d'une néocristide), $371^{0}$ (présence d'un bras postérieur du protoconide) et 3870 (absence d'un mésolophide);

- (7) avec les car. $145^{\circ}$ (présence d'un protolophe labial), $175^{1}$ (présence d'une troisième crête transverse) et $211^{\circ}$ (présence d'un métalophe).

La taille n'a pas été prise en compte dans les analyses, mais le report de ce caractère (inféré à partir de la taille des dents) a posteriori sur la topologie de l'AG1 indique clairement qu'une masse corporelle très réduite serait l'état ancestral chez les caviomorphes (Fig. 7). Il est à noter qu'aucun reste ou fragment dentaire d'espèces de rongeurs de moyenne ou de grande tailles n'est enregistré à ce jour sur l'ensemble des localités éocènes échantillonnées à Contamana, bien que des restes et fragments dentaires de plus grandes taille (attribuables à d'autres groupes de vertébrés) y aient été découverts en nombre. La bunodontie/lophodontie a été considérée avec le car. 1 à trois états: bunodonte, avec des loph(-id)es bas et lophodonte (avec des loph[-id]es hauts). Ces trois états ne permettent pas, toutefois, d'obtenir une résolution aussi fine que pour le (3). Enfin, la plésiomorphie de l'état (8) n'est pas vérifiée (car. 215²), ce caractère étant ambigu entre les états (0) (pas de connexion) et (3) (connexion au postérolophe) à la base du clade Caviomorpha. L'évolution du caractère (8) est difficile à apprécier pour deux raisons: i) sa dilution dans les deux caractères $215^{2}$ (connexion linguale du métalophe avec la troisième crête) et $216^{2}$ (double connexion linguale du métalophe: avec la troisième crête et le postérolophe); et ii) une importante variation et un fort polymorphisme des car. 215 et 216 .

Pozomys ucayaliensis, Tarapotomys mayoensis et T. subandinus sont trois espèces amazoniennes récemment décrites (Boivin et al. 2017a, 2018a). Leur patron dentaire montre des caractères plus dérivés que chez Cachiyacuy et Canaanimys (e.g. tendances à l'hypsodontie, à la taeniodontie et à la réduction du métalophe). Ces caractères sont développés de façon convergente chez les quatre super-familles de caviomorphes, ce qui rendait l'attribution suprafamiliale de ces taxons délicate (famille incertae sedis; Boivin et al. 2017a, 2018a). D'après l'AG1, la convergence de ces caractères (car. 91, 1471, 1511', 


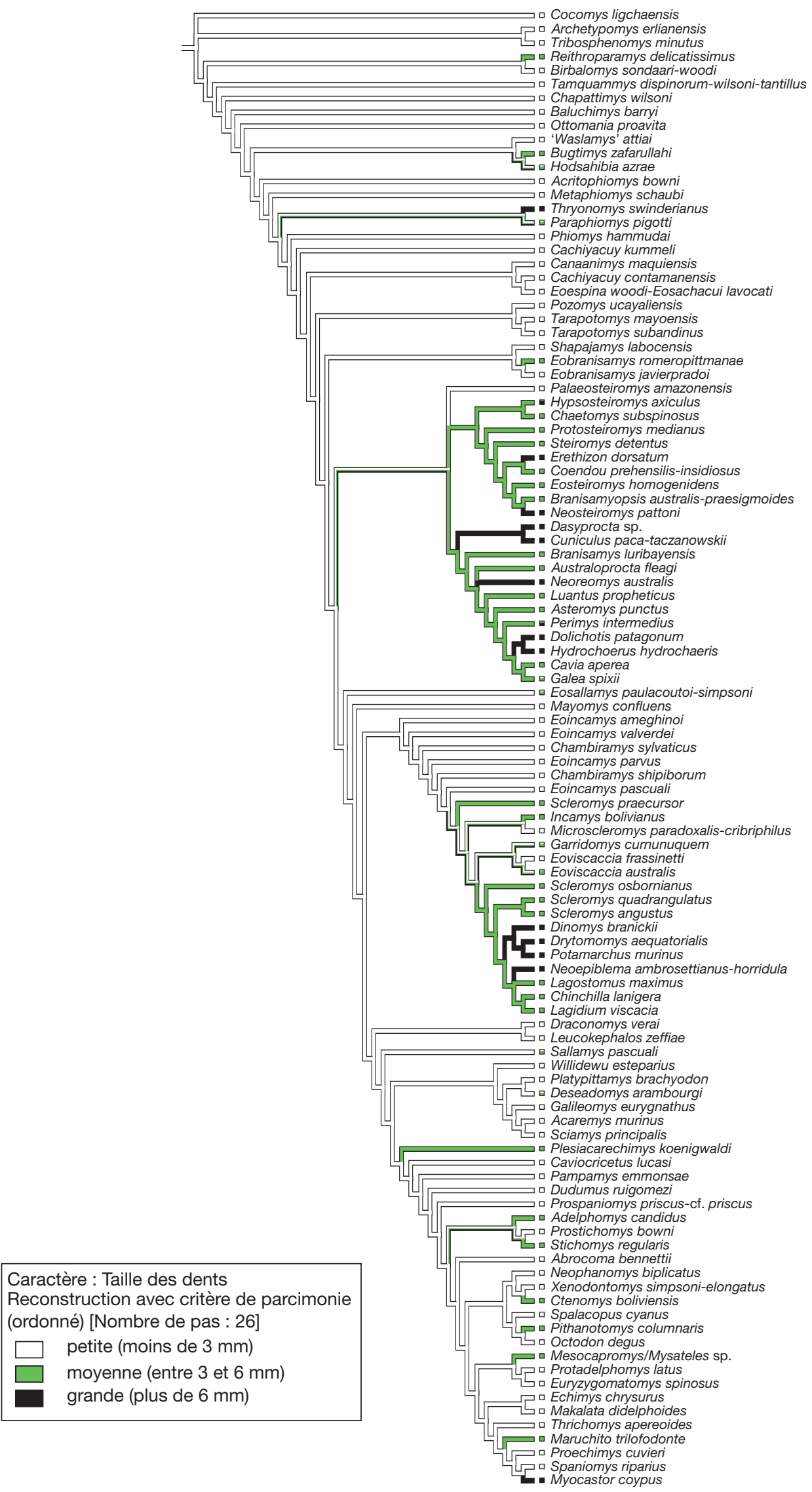

FIG. 7. - Caractère « taille» (i.e., longueur) des dents, reporté a posteriori sur la topologie de l'AG1 (Fig. 5) avec le logiciel Mesquite v.2.72 (Maddison \& Maddison 2009). Ce caractère a été ordonné et a été codé pour l'ensemble des taxons. 
$\left.211^{1}, 212^{1}\right)$ est vérifiée entre certains taxons de différentes super-familles, mais également avec des taxons des caviomorphes basaux tels que Pozomys et Tarapotomys. Dans l'AG7, Plesiosteiromys se branche avec Pozomys, T. mayoensis et $T$. subandinus en raison d'un seul caractère partagé avec $T$. subandinus: un bras postérieur du paracône épais sur les molaires supérieures (car. 1431). Ce caractère n'est retrouvé que chez trois autres caviomorphes (Branisamyopsis, Garridomys et Proechimys). Cependant, d'autres taxons sont polymorphes pour ce caractère: ils montrent un bras postérieur du paracône épais sur certaines de leurs molaires supérieures et fin sur d'autres (C. contamanensis, Steiromys, Eosallamys, Eoincamys valverdei et divers hystricognathes africains). De plus, ce caractère reste peu codé à l'échelle des caviomorphes (chez 29/91 taxons). Plesiosteiromys n'est documenté que par une seule dent, la M2 MUSM 2893 (Boivin et al. 2017b). Ce taxon a originellement été rapproché des éréthizontoïdés, sans contrainte phylogénétique formelle, en raison de l'étendue de ses flexi antérieur et postérieur et d'une ressemblance générale avec Eopululo de Santa Rosa (Frailey \& Campbell 2004). Eopululo est lui aussi documenté par quelques restes dentaires seulement, dont une seule molaire supérieure complète (LACM 143269) qui ne présente aucun métalophe, tout comme MUSM 2893. Néanmoins, l'étendue des flexi n'a pas été prise en compte (voir paragraphe suivant sur Shapajamys) et Eopululo n'a pas été inclus dans l'analyse. Ainsi, la position de Plesiosteiromys à la base des caviomorphes reste incertaine et nécessite d'être confirmée par l'apport de matériel plus abondant documentant ces taxons.

Dans l'étude de la faune de rongeurs de Santa Rosa, Frailey \& Campbell (2004) décrivent pour la première fois le genre Eobranisamys et lui attribuent deux espèces: E. romeropittmanae et $E$. riverai. Récemment, une troisième espèce découverte à CTA-27 lui a été rapportée: E. javierpradoi (Boivin et al. 2017a). Ce genre présente des caractères dentaires similaires à ceux de Branisamys, taxon du Déséadien de Bolivie (Salla; Hoffstetter \& Lavocat 1970; Lavocat 1976). Cela est particulièrement visible au niveau de ses $\mathrm{dP} 4$ et molaires supérieures qui développent un patron taeniodonte et pentalophodonte, caractérisé par une forte troisième crête transverse et un métalophe fort et bien défini. Branisamys a été initialement attribué aux dasyproctidés (i.e., Cavioidea) par Lavocat (1976). Du fait de la relation étroite présumée entre Branisamys et Eobranisamys, ce dernier avait également été assigné à la famille des dasyproctidés (Frailey \& Campbell 2004). Cependant, d'autres auteurs (Patterson \& Wood 1982; Kramarz et al. 2013; Vucetich et al. 2015b) ont remis en question l'appartenance de Branisamys aux caviö̈des, en soulignant des affinités le rapprochant plutôt des dinomyidés ou des chinchilloïdes. Comme souligné précédemment (Boivin et al. 2017a), le statut suprafamilial d'Eobranisamys demeurait jusqu'alors quelque peu ambigu. Outre la position basale au sein des caviomorphes occupée par Eobranisamys dans l'AG1, ce dernier n'est pas associé à Branisamys, qui est placé parmi les caviö̈des non-dasyproctidés (voir section «Les cavioïdes basaux et le cas de Perimys»). La position basale d'Eobranisamys est notamment soutenue par l'absence de certaines synapo- morphies décrivant les Cavioidea (nœud O ; car. 9, 47 et 70), le nœud Q (car. 115) et les Chinchilloidea (car. 183).

Shapajamys labocensis a été précédemment décrit comme appartenant à la famille des éréthizontoïdes sur la base de (1) sa brachyodontie, (2) non-taeniodontie, (3) des flexi(-des) étendus, (4) d'une P4 au contour circulaire, de molaires supérieures (5) pentalophodontes avec (6) un métalophe bien developpé, et (7) une très faible obliquité de ses loph(-id)es (Boivin et al. 2018a). Cependant, les états (1), (5) et (7)sont plésiomorphes au sein des caviomorphes comme vu ci-dessus, de même que l'état (2) correspondant aux caractères 360, $85^{\circ}, 1470,151^{0}, 346^{1}$ et $403^{\circ}$. Ils ne témoignent donc pas de relations étroites avec les éréthizontoïdes qui ont conservé ces caractères primitifs. Les autres états (3), (4) et (6) n'ont malheureusement pas été pris en compte dans l'analyse, leur traduction en caractères à états multiples et leur codage étant difficiles à envisager au vu de la diversité des morphologies observées (avec une multitude de formes intermédiaires) sur l'ensemble des taxons considérés. Shapajamys n'est codé pour aucun des caractères non-ambigus soutenant les éréthizontoïdes (caractères dentaires et crâniens). En revanche, Shapajamys diverge des éréthizontoïdes du nœud N2 par un entoconide postérieurement isolé sur les m1-2 (car. 3970 ; alors qu'il est connecté au postérolophide chez les taxons du nœud N2) et par la présence d'une néocristide 2 sur ses dp4 (car. 3201), une structure seulement retrouvée chez E. romeropittmanae, Branisamys, Luantus et Incamys.

Eoespina woodi et Eosachacui lavocati (considérés ici comme synonymes) ont été originellement attribués aux octodontoïdes (Frailey \& Campbell 2004). Comme pour Eosallamys, un autre genre décrit à Santa Rosa, Frailey \& Campbell (2004) semblent rapprocher Eoespina et Eosachacui de Sallamys pascuali, notamment du fait de la réduction partagée chez ces taxons de la troisième crête transverse des molaires supérieures et de la deuxième cristide transverse des molaires inférieures (respectivement, métalophe et métalophide sensu Frailey \& Campbell [2004]). Sallamys (Bolivie et Pérou; fin Oligocène inférieur-Oligocène supérieur; Hoffstetter \& Lavocat 1970 ; Lavocat 1976; Shockey et al. 2009) a été placé par Patterson \& Wood (1982) au sein des Echimyidae, sous-famille des Heteropsomyinae. À la suite de ces derniers auteurs, Frailey \& Campbell (2004) ont assigné Eosallamys, Eoespina et Eosachacui à cette même famille et sous-famille. La position de Sallamys au sein des échimyidés hétéropsomyinés a été depuis contestée et des analyses cladistiques récentes suggèrent plutôt une parenté proche de Sallamys avec les octodontidés cténomyinés (Verzi et al. 2016), ou les octodontoïdes basaux (Arnal \& Vucetich 2015a). Ce dernier résultat est conforté par notre analyse (voir section «Octodontö̈des: nouvelle définition des Acaremyidae et Adelphomyidae, n. stat., et taxons fossiles au sein du groupe couronne»). En outre, les caractères qui rapprocheraient Eoespina et Eosachacui (et Eosallamys) de Sallamys évoqués par Frailey \& Campbell (2004) n’apparaissent pas valides. En effet, la troisième crête et la deuxième cristide ne sont pas réduites mais sont souvent composées de deux structures différentes: un mésolophe et mésolophule pour les molaires supérieures, et un bras postérieur du protocon- 
ide et un néomésolophide pour les molaires inférieures. En revanche, la présence de molaires supérieures non-taeniodontes, à crêtes transverses et qui tendent à être tétralophodontes (suite à une reduction/absence du métalophe) chez Eoespina et Eosachacui, rappelle le patron typiquement observé chez les octodontoïdes déséadiens (e.g. Platypittamys et Deseadomys) et miocènes (e.g. Dudumus, Caviocricetus et Acaremys). Eoespina et Eosachacui se branchent à la base des octodontoïdes dans les deux autres études phylogénétiques dans lesquelles ils ont été inclus (Arnal \& Vucetich 2015a; Arnal et al. 2017). Toutefois, ces études focalisées sur les relations au sein des octodontoïdes n'ont inclus qu'une dizaine de caviomorphes non-octodontoïdes. D'après l'AG1, la tétralophodontie des molaires supérieures (et des $\mathrm{dP} 4$ ) pouvant être observée chez Eoespina-Eosachacui serait une convergence par rapport à la condition observée chez les octodontoïdes (car. 1150, $211^{1}$ et 2121). Eoespina-Eosachacui ne se branche pas au sein des octodontoïdes ni à la base des (octodontoïdes + chinchilloïdes) en raison de l'absence de nombreux caractères non-ambigus soutenant les nœuds correspondants (nœuds P, Q, R et T) : - la position plus linguale du métacône par rapport au paracône sur les M2 (car. 203² ; ces deux cuspides sont alignées chez Eoespina-Eosachacui);

- un métacône crestiforme sur les M3 (car. $202^{1}$; cette cuspide est distincte chez Eoespina-Eosachacui);

- un bras postérieur du métaconide absent sur les p4 (car. $263^{1}$; structure présente chez Eoespina-Eosachacui);

- un mésostylide, un néomésolophide et un bras antérieur de l'entoconide absents sur les molaires inférieures (car. 3660, $383^{\circ}$ et $396^{\circ}$; structures présentes chez Eoespina-Eosachacui).

Dans l'AG1, les genres Eobranisamys et Tarapotomys sont monophylétiques au contraire de Cachiyacuy: C. kummeli constitue le premier rameau divergeant des caviomorphes (noud B) tandis que C. contamanensis serait plus proche de Eoespina-Eosachacui (nœuds C, puis D). Cependant, les nœuds $\mathrm{C}, \mathrm{D}$ et $\mathrm{E}$ sont soutenus par un seul caractère non-ambigu chacun (car. $38^{2}, 341^{1}$ et $\left.208^{0}\right)$. Cachiyacuy kummeli et $C$. contamanensis étant respectivement codés 0 et $0+1$ pour le caractère 341, leur divergence est surtout liée aux deux autres caractères: 38 (orientation du protolophe lingual sur les P4) et 208 (isolement postérieur du métacône sur les M3).

Le statut de Palaeosteiromys: éréthizontoïde? cavioïde? ou autre? Palaeosteiromys amazonensis est une espèce récemment découverte à Contamana et jusqu'à présent restreinte à la localité CTA-32, datée de l'Oligocène supérieur (Boivin et al. 2017b). Palaeosteiromys a été initialement rapproché des éréthizontoïdes sur les bases de caractères typiques du groupe: un néolophide et des flexides mésiodistalement étendus sur les molaires inférieures, et un métalophe bien développé sur les molaires supérieures (Boivin et al. 2017b).

Dans l'AG1, Palaeosteiromys occupe une position ambiguë: il est phylogénétiquement proche des éréthizontoïdes et des cavioïdes, mais la relation précise avec ces deux groupes est irrésolue. Cependant, sur les huit arbres équiparcimonieux, quatre d'entre eux soutiennent une position basale de Palaeosteiromys au sein des cavioïdes, et les arbres restants présentent ce genre en groupe frère du clade (Erethizontoidea, Cavioidea). Il partage avec ces deux super-familles trois caractères nonambigus (nœuds $\mathrm{N}$ et $\mathrm{O}$; car. $123^{1}, 142^{1}$ et $384^{1}$ ). Le nonrapprochement de Palaeosteiromys avec les éréthizontoïdes et l'irrésolution du nœud $\mathrm{M}$ peuvent être expliqués par:

- la non-inclusion de deux caractères dans les analyses: l'étendue des flexides des molaires inférieures et le développement du métalophe sur les molaires supérieures (comme signalée en section Les groupes basaux des caviomorphes);

- le néolophide (car. 3701) est observé uniquement chez les éréthizontoïdes (dans l'AG1), mais seulement chez quelques espèces du groupe assez éloignées de Palaeosteiromys: Branisamyopsis et Neosteiromys. Un néolophide peut être également présent sur les molaires inférieures de Steiromys, mais cet état est polymorphe chez ce genre. Le développement d'un néolophide chez Palaeosteiromys apparaît ici comme une convergence par rapport à la configuration de Branisamyopsis et Neosteiromys; - Palaeosteiromys n'est pas codé pour trois des quatre caractères non-ambigus soutenant le clade des éréthizontoïdes, et il en diffère pour le quatrième (car. $375^{2}$ au lieu de $375^{\circ} \mathrm{chez}$ les éréthizontoïdes);

- Palaeosteiromys n'est pas codé pour sept des dix caractères non-ambigus soutenant le clade des cavioïdes et il arbore un état différent pour les trois derniers (car. $9^{0}$ au lieu de $9^{1-3}$ chez les cavioïdes, $1466^{1}$ au lieu de $146^{0}$ et $401^{0}$ au lieu de $401^{1}$; le 146 et 401 sont cependant polymorphes au sein des cavioïdes).

\section{Deux familles ou deux sous-familles d'éréthizontoïdes?}

Dans l'AG1 et à l'exclusion de Shapajamys, Palaeosteiromys et Plesiosteiromys, les taxons fossiles précédemment attribués aux éréthizontoïdes sont associés aux représentants actuels du groupe inclus dans l'analyse (Chaetomys, Coendou et Erethizon). Une dichotomie basale des éréthizontoïdes est obtenue avec le regroupement d'Hypsosteiromys avec Chaetomys d'une part (nœud N1), et d'autre part la séquence (Protosteiromys, (Steiromys, ((Coendou, Erethizon), (Eosteiromys, (Branisamyopsis, Neosteiromys))))) ; nœud N2). Ces deux ensembles correspondent ainsi aux deux sous-familles des éréthizontoïdes, Chaetomyinae et Erethizontinae (au sein de la famille des Erethizontidae), définies à partir des représentants actuels de la super-famille (Patton et al. 2015). En effet, la divergence précoce de Chaetomys et sa différenciation morphologique visà-vis des autres éréthizontoïdes actuels justifient l'établissement d'une sous-famille (Martin 1994; Woods \& Kilpatrick 2005), voire d'une famille (Upham \& Patterson 2015) à partir de ce genre. L'analyse de la distribution des caractères dans l'AG1 révèle en fait une distinction assez peu marquée entre les deux clades (N1 et N2), ce qui suggèrerait plutôt une correspondance de ces nœuds au rang sous-familial:

- un nombre assez faible de caractères non-ambigus soutenant chaque nœud (cinq pour N1 et 2 pour N2);

- sur les cinq caractères non-ambigus soutenant le nœud N1, trois sont retrouvés de façon convergente chez au moins un taxon du groupe N2 (Steiromys, Branisamyopsis et Neosteiromys); - pour l'un des deux caractères non-ambigus soutenant le nœud N2 (3971), l'état 1 est également retrouvé chez Hypsosteiromys, qui est polymorphe pour ce caractère $(3970+1)$; 
- pour le second caractère soutenant le nœud N2 (1970), une réversion (1971) est possible pour certains taxons issus de ce noud (Erethizon et Branisamyopsis).

Les relations obtenues au sein des éréthizontoïdes dans l'AG1 divergent des résultats de Candela (2004), fondés sur Candela $(2000,2001)$, ces derniers montrant une dichotomie basale entre les Erethizontinae (Erethizon, Coendou) et les Steiromyinae (Hypsosteiromys, (Neosteiromys, (Branisamyopsis (Eosteiromys, Steiromys)))). Les différences topologiques (i.e., position d'Hypsosteiromys, relations des autres taxons fossiles entre eux et avec Erethizon-Coendou) entre les deux analyses peuvent être expliquées par:

- l'inclusion de Chaetomys dans l'AG1, taxon non pris en compte par Candela (2004);

- des groupes externes différents et la prise en considération d'un nombre plus important de taxons dans l'AG1 (caviomorphes non-éréthizontoïdes);

- des jeux de caractères différents : celui de l'AG1 est fortement dominé par les traits dentaires, tandis que celui de Candela (2004) semble plus axé sur la morphologie cranio-mandibulaire.

\section{Les cavioïdes basaux et le cas de Perimys}

Tous les fossiles étroitement apparentés aux taxons modernes de cavioïdes divergent basalement aux caviidés dans l'AG1 selon la séquence (Branisamys, (Neoreomys, (Australoprocta, (Luantus, (Asteromys, Perimys (caviidés modernes)))))).

Branisamys et Australoprocta ont été originellement attribués aux dasyproctidés (Lavocat 1976; Kramarz 1998). Comme précédemment évoqué (voir section «Les groupes basaux des caviomorphes»), l'attribution de Branisamys aux dasyproctidés a été contestée par plusieurs auteurs (Hartenberger 1975; Patterson \& Wood 1982; Kramarz et al. 2013; Vucetich et al. 2015b). Sans plus amples explications, Hartenberger (1975) rapprochait Branisamys des dinomyidés (Hartenberger 1975: pl. 1, fig. 1). Ce rapprochement fut formalisé par Patterson \& Wood (1982), qui plaçaient alors Branisamys au sein des dinomyidés, du fait de sa morphologie crânienne similaire à celle de Dinomys (Patterson \& Wood 1982: 446, tableau 7). Selon ces auteurs, le passage du plan dentaire de Branisamys vers celui de Dinomys, bien qu' entraînant plusieurs modifications (e.g. élévation de la couronne, élongation des dents, etc.), aurait été possible en considérant une morphologie dentaire proche de celle de Drytomomys aequatorialis. Patterson \& Wood (1982) ont noté toutefois des ressemblances entre Branisamys et les dasyproctidés, qui témoigneraient d'une parenté moins directe, et positionnaient ainsi les dinomyidés (Branisamys inclus) et les dasyproctidés au sein d'une même super-famille, celle des cavioïdes. L'apparentement des dasyproctidés aux cavioïdes est fortement soutenu par des études phylogénétiques fondées sur des données moléculaires, au contraire de celui des dinomyidés (aujourd'hui restreints au seul Dinomys branickii), qui présentent d'étroites relations avec les chinchillidés (e.g. Fabre et al. 2012; Upham \& Patterson 2015). Suivant Patterson \& Wood (1982) et prenant en compte ces données moléculaires, Kramarz et al. (2013) ont considéré Branisamys comme étant un dinomiydé chinchilloïde. Ces auteurs signalent que Branisamys montre une P4 entièrement molarisée comme chez les autres chinchilloïdes (excepté les deux néoépiblemidés présumés, Scotamys et Perimys), et présenterait des caractères moins dérivés que les autres représentants du groupe, tels qu'une couronne dentaire plus basse et une homogénéité de l'épaisseur de l'émail des bords d'attaque et de fuite des dents jugales. Néanmoins, l'analyse cladistique opérée par Kramarz et al. (2013), fondée sur 39 caractères dentaires, crâniens et mandibulaires, ne soutient pas une parenté de Branisamys avec les dinomyidés, ce genre divergeant à la base des chinchilloïdes. En outre, cette analyse n'a pris en compte aucun des membres avérés des trois autres super-familles, mis à part l'octodontoïde fossile Prospaniomys, utilisé comme seul groupe externe. Dans l'AG1, la position de Branisamys à la base des caviidés peut être expliquée par: - la dissemblance de Branisamys pour la majorité des caractères non-ambigus soutenant le nœud O1 (Dasyprocta, Cuniculus) : car. $254^{2}, 4370,472^{\circ}$ et $473^{\circ}$. Branisamys n'est pas codé pour deux d'entre eux (car. 455? et 503?) et montre un état polymorphe pour le dernier (car. 3670+1);

- la dissemblance de Branisamys pour 10/25 des caractères non-ambigus de Dasyprocta: car. 222 , 351, 2132, 2231, $227^{0}$, $229^{\circ}, 231^{0}, 410^{0}, 414^{0}$ et $460^{\circ}$. Branisamys n'est pas codé pour onze d'entre eux (car. 20?, 375?, 376?, 433?, 444?, 450?, 451?, 467?, 470?, 485? et 509?). Il ne montre que $2 / 25$ caractères communs à Dasyprocta: une néocristide parfois présente dans l'antéroflexide sur les p4 (car. 2681) et un bras postérieur du protoconide long sur les m1-2 (car. 3720);

- la divergence de Branisamys pour 2/6 des caractères nonambigus des chinchilloïdes : présence d'un mésolophule sur les M1-2 (car. 1771) et un mésolophe connecté au mésolophule sur les M1-2 (car. 1831). Branisamys n'est pas codé pour deux d'entre eux (car. 153? et 255?);

- la dissemblance de Branisamys pour $1 / 3$ des caractères nonambigus soutenant le nœud $\mathrm{R}$ (Chinchilloidea, Octodontoidea) : un métacône distinct sur les M3 (car. 2021) alors que cette cuspide, lorsqu'elle est présente, est crestiforme chez les chinchilloïdes;

- Branisamys montre les cinq caractères non-ambigus soutenant le nœud O2. Parmi ces cinq caractères, la présence d'un mésolophide sur les m3 (car. 3891) est exclusivement rencontrée, au sein des caviomorphes, chez les représentants de ce groupe, Dasyprocta et Coendou. Cependant, certains chinchilloïdes partagent les quatre autres de façon convergentes (car. $3^{2}, 222^{0}, 374^{1}$ et $461^{2}$ ).

Les trois caractères non-ambigus des dinomyidés sont tous partagés par Branisamys. Toutefois, chez les dinomyidés, deux de ces caractères (car. $211^{0}$ et $409^{\circ}$ ) sont des réversions. En effet, cette famille montre une morphologie pour ces deux caractères qui ressemble à la condition observée chez les caviomorphes basaux. De plus, d'après l'AG1, une courte excroissance du protocône sur les P4 (car. 311), qui traduit la molarisation des P4 évoquée par Kramarz et al. (2013), se développerait de manière convergente entre les Erethizontoidea-Cavioidea, certains Chinchilloidea (représentants du nœud S5) et certains Octodontoidea (Galileomys, Acaremys et Sciamys). Les cavioïdes développeraient secondairement une excroissance longue du protocône sur les P4 (car. 310; réversion). 
Kramarz (1998) assigne Australoprocta (Formation Sarmiento, Argentine; Colhuehuapien, Miocène inférieur) aux dasyproctidés sur la base: (1) d'une couronne dentaire haute à croissance limitée, (2) de dents quadrangulaires qui deviennent plus larges que longues avec l'usure, (3) de vallées étroites assez persistantes, (4) d'un plan pentalophodonte des molaires supérieures et (5) de la présence d'une crestule accessoire dans le paraflexus. Selon Kramarz (1998), bien qu'il possède certains caractères apparaissant plus dérivés que chez Dasyprocta (i.e., des molaires supérieures non complètement pentalophodontes et des loph [-id] es plus obliques), Australoprocta serait plutôt un représentant basal des dasyproctidés du fait d'une couronne moins haute que Dasyprocta, de P4 non molarisées et de M3 divergeantes des M1-2. Les caractères (1), (3) et (4) ont été pris en compte dans l'AG1:

- (1) avec le car. $9^{1}$ ou 2 (Australoprocta est codé $9^{1}$ tandis que Dasyprocta est codé 92);

- (3) avec les car. 2270 ou 1 (paraflexus), $229^{0}$ ou 1 (mésoflexus mésial), $231^{1}$ (postéroflexus), 410 (antéroflexide), $412^{1}$ (mésoflexide) et $414^{1}$ (métaflexide);

- (4) avec les car. $145^{\circ}$ (présence d'un protolophe labial), $175^{1}$ (présence d'une troisième crête transverse) et $211^{0}$ (présence d'un métalophe).

Le caractère (1) est trouvé chez les dasyproctidés mais également chez certains caviidés fossiles tels que Asteromys (codé $9^{1}$ dans l'AG1) ou Luantus (codé $9^{2}$ dans l'AG1). Comme le signale Kramarz (1998), la pentalophodontie des molaires supérieures (3) est un caractère ancestral chez les caviomorphes (voir section "Les groupes basaux des caviomorphes»), de même que chez les cavioïdes et les caviidés. Ainsi, ces deux caractères (1) et (3) ne permettent pas de regrouper Australoprocta avec les dasyproctidés de manière certaine. Pour le caractère (2), Dasyprocta possède des flexi plus persistants que chez certains caviidés (e.g. Luantus), mais sur l'ensemble des taxons observés dans cette analyse et au niveau d'usure considéré pour les caractères 227, 229 et 231, les flexi de Dasyprocta se réduisent modérément $\left(222^{2}\right)$ ou fortement (2272 et 2311). Au même degré d'usure (notamment basé sur celui de la MACN CH 1776; Kramarz 1998: figure 3E, p. 184), Australoprocta semble avoir un paraflexus et un mésoflexus mésial peu réduits $\left(227^{2}\right.$ et $\left.229^{2}\right)$, tandis que son postéroflexus a disparu $\left(230^{\circ}\right)$, des caractères qui participent sans doute à la dissemblance d'Australoprocta avec Dasyprocta. De même, pour les molaires inférieures, Dasyprocta possède un métaflexide très réduit (4141) alors qu'Australoprocta semble avoir un métaflexide encore bien développé (4140) à un stade d'usure comparable. Toutefois, Australoprocta n'étant connu que par des dents isolées, l'évolution ontogénétique du patron de ses molaires supérieures est difficilement appréciable et a pu être ici mal interprété. Dans l'AG1, Australoprocta diverge de Dasyprocta:

- pour 7/25 des caractères non-ambigus de Dasyprocta: car. $35^{1}, 213^{2}, 2270,229^{0}, 372^{1+2}, 373^{2}$ et 4140 . Australoprocta n'est pas codé pour quinze d'entre eux (car. 20?, 22?, 231?, 268 ? 375 ? , 376?, 433?, 444?, 450?, 451?, 460?, 467?, 470?, 485? et 509?). Il ne montre que $2 / 25$ caractères communs à Dasyprocta: un postérolophe peu ou pas connecté à l'hypocône sur les M3 (car. 2230) et une forte réduction de l'antéroflexide sur les molaires inférieures (car. 4101);

- notamment par une longue excroissance du protocône, qui se connecte à l'hypocône, sur les P4 (i.e., P4 non molarisée; $31^{0}$ ), tandis que cette structure est courte (non connectée à l'hypocône) chez Dasyprocta.

La position de Perimys au sein des cavioïdes obtenue dans l'AG1 est à première vue très surprenante. En effet, ce genre, retrouvé dans diverses formations géologiques d'Argentine datées du Miocène inférieur (formations Sarmiento, Santa Cruz, Pinturas et Cerro Boleadoras; Colhuehuapien et Santacrucien SALMAs) et au Chili (Pampa Castillo; Santacrucien et Friasien), développe un patron dentaire très simplifié, rappelant fortement celui des chinchilloïdes actuels (et particulièrement Lagostomus) ou de représentants fossiles de cette super-famille (e.g. Phoberomys, Neoepiblema et Eoviscaccia). Du fait d'un patron dentaire très ressemblant à ces derniers, Perimys a été placé de façon récurrente dans les Chinchilloidea (Miller \& Gidley 1918; Simpson 1945; Schaub 1953, 1958; Landry 1957; Woods 1984). Certains auteurs l'ont placé au sein d'une famille de chinchilloïdes éteints, les néoépiblemidés (e.g. Ameghino 1887; McKenna \& Bell 1997; Flynn et al. 2002; Kramarz 2002), une relation récemment soutenue par deux analyses cladistiques (Rasia \& Candela 2017; Kerber et al. 2018). Ces analyses, fondées sur le travail de Kramarz et al. (2013), se concentrent sur les relations phylogénétiques des chinchilloïdes et n'incluent que Prospaniomys en groupe externe. La position de Perimys au sein des cavioïdes dans l'AG1 peut être expliquée par plusieurs facteurs:

- aucun caractère crânien ou mandibulaire n’a été contrôlé pour Perimys, ce dernier ayant été codé sur la base de $P$. intermedius, essentiellement connu par des restes dentaires;

- d'innombrables convergences entre les cavioïdes et les chinchilloïdes qui peuvent brouiller le signal. Sur l'ensemble des caractères non-ambigus des différents nœuds qui soutiennent son affiliation aux caviö̈des, Perimys montre des états convergents avec les taxons du groupe couronne des chinchilloïdes pour la majorité (18/32; Perimys non codé pour 11 d'entre eux);

- une grande variation du patron des prémolaires définitives de Perimys, notamment ses $\mathrm{P} 4$, par rapport à celui des taxons du groupe couronne des chinchilloïdes. Perimys diverge notamment de ces taxons par une longue excroissance du protocône sur les $\mathrm{P} 4$ (car. 310), tandis que cette structure est courte chez le groupe couronne des chinchilloïdes;

- une interprétation particulière des homologies dentaires chez les chinchilloïdes. Chez les représentants de cette superfamilles, les loph(-id)es sont généralement fortement obliques. Chez les dinomyidés, ils tendent à s'individualiser formant ainsi des lamines parallèles (car. 2441-2481 ; e.g. Drytomomys) et certains membres de cette famille peuvent développer des lamines surnuméraires, notamment sur les $\mathrm{M} 3 / \mathrm{m} 3$ (e.g. Potamarchus, Eumegamys). Les dinomyidés montrent souvent avec l'usure une perte de loph(-id)es ou lamines (due à la fusion de deux loph(-id)es ou lamines, à l'avant pour les dents inférieures et à l'arrière pour les dents supérieures; e.g. Scleromys, Drytomomys, Potamarchus). Les patrons dentaires 
des dents jugales de certains néoépiblémidés (Neoepiblema et Phoberomys) et de certains genres actuels (Dinomys, Chinchilla et Lagidium), ressemblant fortement à ceux des dinomyidés, la formation de lamines est considérée chez ces genres. Un autre phénomène assez similaire est observé chez les caviomorphes: des loph(-id)es fusionnent avec l'usure et forment des structures, nommées ici lobes, souvent plus longs antéropostérieurement que les lamines, moins obliques, généralement peu parallèles et peu répétés (car. $235^{1}$ et 4181 ). La formation de lobes caractérise notamment les dents des cavioïdes (e.g. Asteromys, Luantus, Eocardia et les caviidés actuels) et elle est accompagnée d'une extension transverse de l'hypoflexus et de l'hypoflexide (car. $234^{1}$ et 4161). D'autres taxons tels que Cephalomys, Banderomys, Scotamys, Litodontomys, Eoviscaccia, les cténomyidés ou les octodontidés montrent la formation de lobes. L'analyse du matériel attribué à Perimys intermedius et notamment des dents les plus fraîches, semble indiquer le développement de lobes chez ce taxon, associé à une extension transverse de l'hypoflexus et de l'hypoflexide. L'extension des ces flexi(-des) n'étant observée que chez les cavioïdes, ces caractères soutiennent le rapprochement de Perimys avec les cavioïdes, obtenu dans l'AG1.

Ces interprétations concernant la formation de lamines/ lobes chez les chinchilloïdes et les caviomorphes en général demeurent toutefois du domaine de l'hypothèse. La limite entre ces deux conditions, lamines/lobes, peut être ambiguë chez certains taxons comme Garridomys ou Eoviscaccia, ce qui n'exclut pas d'autres assomptions (voir Kramarz et al. 2013). Comme le notent Kramarz et al. (2013), les homologies entre les structures occlusales des chinchilloïdes paléogènes-miocènes et celles des chinchilloïdes modernes (et des taxons fossiles qui leur sont directement apparentés) sont incertaines en raison de registres paléontologiques et des trajectoires ontogénétiques incomplets, et ne permettent pas de privilégier pour l'instant une hypothèse plutôt que d'autres.

Dans ses travaux de thèse, Pérez (2010b) a effectué une étude cladistique qui a permis une meilleure compréhension des relations phylogénétiques et de l'évolution des "éocardiidés", et plus généralement des caviidés (travaux formalisés par Pérez 2010a; Pérez \& Vucetich 2011, 2012a, b; Pérez \& Pol 2012; Pérez et al. 2012). Les trois taxons précédemment discutés (Branisamys, Australoprocta et Perimys) ne sont pas inclus dans ces travaux, au contraire de Neoreomys (formations Sarmiento et Pinturas, Argentine; Miocène inférieur), qui était auparavant souvent considéré comme un dasyproctidé (e.g. Miller \& Gidley 1918; McKenna \& Bell 1997), Luantus (formations Sarmiento et Pinturas, Argentine; Laguna del Laja, Chili ; Miocène inférieur) et d'Asteromys (Cabeza Blanca, Laguna de los Machos, Argentine; fin Oligocène inférieur-Oligocène supérieur). La position de ces trois derniers genres comme caviidés basaux et la divergence basale de Neoreomys dans l'AG1 sont en accord avec les travaux correspondants (Pérez 2010a, b; Pérez \& Vucetich 2011, 2012a, b; Pérez \& Pol 2012; Pérez et al. 2012). Cependant, Luantus diverge avant Asteromys dans l'AG1, tandis qu'une relation inverse, plus en conformité avec les données stratigraphiques, est obtenue dans les travaux de Pérez (et cela pour les trois espèces inclues de Luantus qui divergent après Asteromys). Dans l'AG1, le patron occlusal des p4 de Luantus apparaît plus primitif que celui d'Asteromys pour trois caractères: la présence d'un métalophulide I (car. 2590) et d'un bras antérieur du métaconide (car. 260), deux structures absentes chez Asteromys, et l'absence de cristide/éperon du métaconide (car. 2670), qui est au contraire présent chez Asteromys. Luantus a été contrôlé à partir du matériel attribué à L. propheticus figuré par Kramarz (2006a: 772, 773, figs 2, 3). Chez les formes hypsodontes comme Luantus, la présence d'un métalophulide I (car. 2590), d'un bras antérieur du métaconide (car. 2600) et d'un bras antérieur du protoconide (car. 2610) est potentiellement liée à l'usure, ces structures pouvant être absentes sur des germes et apparaître à un stade d'usure plus avancé. Toutefois, l'analyse des $\mathrm{p} 4 \mathrm{de} L$. propheticus à divers stades d'usure (Kramarz 2006a: 773, fig. 3) semble indiquer la présence d'un bras antérieur du métaconide et donc d'un métalophulide I, alors que la présence d'un bras antérieur du protoconide semble plus variable (Luantus est codé $0+1$ pour ce caractère). Ces observations nécessitent d'être confirmées avec un nombre de spécimens plus conséquent qui permettait une meilleure compréhension des stades ontogénétiques de Luantus.

\section{Eosallamys et Mayomys}

Deux taxons apparaissent en groupes basaux du clade (Chinchilloidea, Octodontoidea) dans l'AG1: Eosallamys (Santa Rosa, Pérou, ?Éocène supérieur/Oligocène inférieur; Frailey \& Campbell 2004) et Mayomys (TAR-01, Pérou, Oligocène inférieur; Boivin et al. 2018a).

Suivant le même raisonnement que pour Eoespina et Eosachacui (voir section "Les groupes basaux des caviomorphes»), l'attribution d'Eosallamys aux échimyidés hétéropsomyinés par Frailey \& Campbell (2004) est remise en cause. Dans leur étude visant à une meilleure compréhension des grandes radiations des rongeurs octodontoïdes au Paléogène et Miocène, Arnal \& Vucetich (2015a) et Arnal et al. (2017) ont inclus Eosallamys dans leur analyse cladistique. Les résultats obtenus par ces auteurs soutiennent une position d'Eosallamys en octodontoïde basal. Pourtant, contrairement aux patrons dentaires d'Eoespina et d'Eosachacui, qui montrent une similarité apparente avec les octodontoïdes (voir section «Les groupes basaux des caviomorphes»), celui d'Eosallamys apparaît clairement éloigné des membres de cette super-famille, et rappelle plus celui de taxons considérés comme primitifs au sein des caviomorphes (Cachiyacuy et Canaanimys) en raison de: sa brachyodontie, des molaires inférieures tétralophodontes avec une deuxième cristide bien développée, des molaires supérieures pentalophodontes avec une troisième crête transverse, et un métalophe bien développés, pouvant être connectés lingualement (Boivin et al. 2017a). Cependant, la plésiomorphie de ce dernier trait n'est pas confirmée dans l'AG1 à l'inverse des autres caractères (voir section «Les groupes basaux des caviomorphes»), et Eosallamys présente deux caractères dérivés à l'échelle des caviomorphes, qui le rapprochent en fait du clade (Chinchilloidea, Octodontoidea) dans l'AG1 : un métacône absent sur les P4 (car. 641) et un métacône positionné plus lingualement au paracône sur les M2 (car. 2032). 
Le genre Mayomys, récemment décrit à TAR-01, a initialement été assigné aux octodontoïdes (Boivin et al. 2018a). Cette attribution repose sur plusieurs ressemblances entre Mayomys et des octodontoïdes paléogènes (e.g. Platypittamys, Paulacoutomys) et miocènes (e.g. Dudumus) telles que la tétralophodontie des $\mathrm{dP} 4$ et M1-2, résultant de l'absence de métalophe, la forme allongée des $\mathrm{dP} 4$ et la réduction de la seconde cristide (i.e., bras postérieur du protoconide court et absence de néomésolophide) des molaires inférieures. D’après les résultats de l'AG1, les absences du métalophe sur les dP4 (car. 1150) et sur les M1-2 (car. 2111) seraient des caractères dérivés partagés avec les chinchilloïdes, alors que la forme allongée des $\mathrm{dP} 4\left(7^{2}\right)$ et un bras postérieur du protoconide court (3721) seraient des états ancestraux observés chez d'autres caviomorphes. L'évolution de la longueur du bras postérieur du protoconide des m1-2 est néanmoins difficile à apprécier en raison d'une forte variation intertaxonomique et intrataxonomique (polymorphisme). L'absence de néomésolophide sur les molaires inférieures (car. 3830) est l'un des caractères non-ambigus soutenant le clade des octodontoïdes (nœud T). Mayomys montre, toutefois, un polymorphisme pour ce caractère (codé $383^{0+1}$ ), ainsi que pour deux caractères nonambigus du clade $T$ : un bras postérieur du métaconide court (car. 3601), et l'absence à la fois du mésostylide (car. 366º) et du bras antérieur de l'entoconide (car. 3960) sur les molaires inférieures. La non-inclusion de Mayomys au sein des octodontoïdes s'explique surtout par la morphologie de sa p4, qui apparaît primitive pour certains caractères: un bras antérieur du métaconide connecté à celui du protoconide (car. 2621; libre chez les octodontoïdes); la présence d'un bras postérieur du métaconide (car. $263^{\circ}$; absent chez les octodontoïdes) et d'un mésostylide (car. 2651). De plus, il n'y a pas de réduction importante de l'antéroflexide sur les molaires inférieures de Mayomys (car. 410º), contrairement aux octodontoïdes.

\section{Les chinchilloïdes basaux}

Plusieurs taxons sont situés à la base des chinchilloïdes dans l'AG1 :

- les espèces récemment décrites de l'Oligocène supérieur de Contamana (CTA-61 et CTA-32: Chambiramys shipiborum, Chambiramys sylvaticus et Scleromys praecursor; Boivin et al. 2017b) et de l'Oligocène inférieur de la région de Tarapoto/ Shapaja (Eoincamys valverdei et Eoincamys parvus; Boivin et al. 2018a) ;

- les deux espèces d'Eoincamys découvertes à Santa Rosa (Pérou, ?Éocène supérieur/Oligocène inférieur) : E. ameghinoi et E. pascuali (Frailey \& Campbell 2004);

- Incamys bolivianus de Salla (Bolivie, fin Oligocène inférieurOligocène supérieur; Hoffstetter \& Lavocat 1970; Lavocat 1976; Patterson \& Wood 1982) associée ici à Microscleromys de La Venta (Colombie, fin Miocène moyen; Walton 1997); - Garridomys de la Formation Cerro Bandera (Argentine, Miocène inférieur; Kramarz et al. 2013) associé aux deux espèces d'Eoviscaccia: E. frassinettii (Termas del Flaco, Chili, Oligocène inférieur; Bertrand et al. 2012) et E. australis (formations Sarmiento, Cerro Bandera et Chinchinales, Miocène inférieur; Kramarz 2001b; Vucetich et al. 2010b).
- les espèces de Scleromys des formations Santa Cruz et Pinturas (Argentine) datées de la fin du Miocène inférieur: S. osbornianus (Ameghino 1894; Kramarz 2006b), S. angustus (Ameghino 1887) et $S$. quadrangulatus (Kramarz 2006b).

Comme Branisamys (voir section «Les cavioïdes basaux et le cas de Perimys»), Incamys a été originellement attribué aux cavioïdes et à la famille des dasyproctidés (Lavocat 1976). Dans leur révision de la faune de Salla, Patterson \& Wood (1982) ont signalé une divergence importante entre la morphologie crânienne de Branisamys et celle d'Incamys, malgré le fait que ces deux taxons se ressemblent fortement d'un point de vue dentaire. Ces auteurs ont assigné Branisamys aux dinomyidés, tandis qu'Incamys est resté assigné aux dasyproctidés, comme initialement proposé par Lavocat (1976). Cependant, Vucetich et al. (2015b) ont récemment remis en question cette assignation et ont rapproché Incamys des chinchilloïdes du fait d'une morphologie différente du foramen incisif entre Incamys et les dasyproctidés modernes. De plus, Incamys montre des affinités avec les chinchilloïdes et particulièrement avec les chinchillidés au niveau de son patron dentaire, caractérisé par une réduction des loph(-id)es et une hétérogénéité de l'épaisseur de l'émail entre les bords d'attaque et de fuite (Vucetich et al. 2015b). Une relation récemment soutenue par l'analyse cladistique réalisée par Busker \& Dozo (2018) dan laquelle l'espèce Incamys bolivianus est plus proche du chinchilloïde Eoviscaccia que des taxons cavioïdes inclus dans l'analyse. Le résultat obtenu dans l'AG1 est en adéquation avec Vucetich et al. (2015b) et Busker \& Dozo (2018). L'appartenance d'Incamys aux chinchilloïdes dans l'AG1 repose notamment sur le fait qu'Incamys présente plusieurs caractères non-ambigus de la super-famille: - une protohypsodontie (car. 92);

- une épaisseur hétérogène de l'émail de l'hypoflexus et hypoflexide sur les molaires (car. 131);

- une tendance à la taeniodontie sur les P4 (car. 361), les M1-3 (car. $147^{1}$ et 1511) et les molaires inférieures (car. 4031); - une tendance à une connexion linguale du mésolophe avec le postérolophe sur les M1-2 (car. 1834);

- une tendance à la perte du métacône sur les M1-2 (car. 1991); - un protoconide plus développé que le métaconide sur les p4 (car. 2562);

- une perte de la connexion linguale du métalophulide I avec le métaconide sur les p4 (car. 3110).

Le genre Eoincamys a été décrit à Santa Rosa avec E. pascuali comme espèce type et $E$. ameghinoi comme espèce assignée (Frailey \& Campbell 2004). De par les ressemblances entre Eoincamys et Incamys (i.e., tendances à la taeniodontie sur les molaires supérieures et inférieures, à une connexion linguale du mésolophe réduit avec le postérolophe, à la perte du métacône sur les M1-2 et à une réduction du néomésolophide sur les molaires inférieures), Frailey \& Campbell (2004) ont assigné Eoincamys aux dasyproctidés. À l'occasion de la description de deux nouvelles espèces attribuées à Eoincamys (E. valverdei et $E$. parvus), le statut suprafamilial de ce genre a été discuté et, comme pour Incamys, un rapprochement avec les chinchilloïdes a été envisagé (voir Boivin et al. 2018a). Une affinité Eoincamys-chinchilloïdes est soutenue par l'AG1, et cela pour les quatre espèces du genre. 
Le genre Chambiramys a été récemment décrit (Boivin et al. 2017b) à CTA-32 (avec C. shipiborum) et à CTA-61 (avec C. sylvaticus), dans l'Oligocène supérieur de Contamana. Chambiramys sylvaticus présente les mêmes caractères qu' Incamys et Eoincamys, et se rapproche notamment d'E. pascuali par la forte obliquité des loph(-id)es sur les molaires. Chambiramys shipiborum montre les mêmes tendances que C. sylvaticus et E. pascuali, mais avec une tendance plus marquée; ses molaires supérieures sont caractérisées par l'absence du mésolophe et la seule molaire inférieure (MUSM 2846) qui lui est attribuée par l'absence du néomésolophide. Plusieurs de ces traits (i.e., taeniodontie des molaires supérieures et inférieures, connexion linguale du mésolophe réduit avec le postérolophe et une réduction du néomésolophide sur les molaires inférieures) se retrouvent également chez d'autres taxons comme les chinchilloïdes Scleromys et Drytomomys, le dasyproctidé présumé Microscleromys (voir Walton 1997) et chez l'octodontoïde Protadelphomys. Au vu de ces apparentes convergences et du précédent statut suprafamilial incertain d'Incamys et d'Eoincamys, Chambiramys n'a originellement été attribué à aucune super-famille ("super-famille incertae sedis»; Boivin et al. 2017b). Comme pour Incamys et Eoincamys, les deux espèces de Chambiramys et Microscleromys divergent au sein des chinchilloïdes dans l'AG1. Les affinités de Protadelphomys avec les octodontoïdes sont soutenues dans l'AG1 et les caractères similaires entre Protadelphomys et ces divers taxons sont interprétés comme des convergences.

Dans l'AG1, les quatre espèces d'Eoincamys ne forment pas un groupe monophylétique, pas plus que les deux de Chambiramys, ces six espèces divergeant successivement à la base des chinchilloïdes. La position de ces taxons dans l'AG1 reflète l'accentuation des tendances précédemment discutées, avec à la base $E$. ameghinoi qui montre des loph(-id)es peu obliques, un mésolophe réduit lingualement libre ou tourné vers le postérolophe mais sans connexion avec ce dernier, et un néomésolophide pouvant encore être connecté à l'ectolophide. Eoincamys pascuali diverge en dernier. Bien que présentant un patron dentaire plutôt intermédiaire entre $E$. ameghinoi et $E$. pascuali, Incamys diverge après $E$. pascuali. Cette position s'explique par le fait que trois traits plus dérivés sont présents chez Incamys: des dents protohypsodontes avec des loph(-id)es très épais et une épaisseur hétérogène de l'émail de l'hypoflexus et de l'hypoflexide sur les molaires. Au contraire, les espèces d'Eoincamys et de Chambiramys sont caractérisées par des dents brachyodontes ou mésodontes avec des loph(id)es moins épais, et une épaisseur homogène de l'émail de l'hypoflexus et de l'hypoflexide sur les molaires.

Après avoir été attribué successivement aux échimyidés (Ameghino 1889), aux myocastoridés (Scott 1905) et aux dasyproctidés (Patterson \& Wood 1982), le genre Scleromys est à l'heure actuelle considéré comme un dinomyidé par de nombreux auteurs (e.g. Fields 1957; Walton 1997; Kramarz 2006b). L'appartenance de Scleromys aux dinomyidés est soutenue par des études cladistiques récentes (Kramarz et al. 2013; Kerber et al. 2017, 2018; Rasia \& Candela 2017). Dans l'AG1, aucune des quatre espèces de Scleromys inclues dans l'analyse ( $S$. osbornianus, $S$. angustus, $S$. quadrangulatus et $S$. praecursor) ne se branchent au sein des dinomyidés, lesquels s'avèrent ici plus étroitement apparentés aux chinchillidés (Chinchilla, Lagidium et Lagostomus) et néoépiblemidés (Neoepiblema). Les espèces de Scleromys ne partagent pas la majorité des caractères non-ambigus soutenant le groupe couronne des chinchilloïdes (nœud S8) et ceux soutenant les dinomyidés (noud S9) :

- une hétérogénéité de l'épaisseur de l'émail importante sur les molaires (car. 141), alors qu'elle est moins prononcée chez Scleromys;

- un métalophe présent sur les M1-2 (car. 2110), alors qu'il est absent chez Scleromys;

- des lamines présentes sur les M1-2 (car. 2441), les M3 (car. $245^{1}$ ) et sur les molaires inférieures (car. 4261), tandis qu'elles sont absentes chez Scleromys;

- un antéroflexide présent sur les molaires inférieures (car. $409^{0}$ ), alors qu'il est absent chez Scleromys;

- un diastème inférieur court (car. 4341), alors qu'il est encore plus court chez Scleromys;

- une échancrure où s'insère le tendon du masseter medialis pars infraorbitalis, située sous la $\mathrm{m} 1$ (car. $440^{1}$ ), tandis qu'elle est positionnée sous la $\mathrm{p} 4$ ou entre la p4-m1 chez Scleromys; - une origine de la crête massétérique sous la m2 (car. 44211), tandis qu'elle est sous la $\mathrm{m} 1 \mathrm{chez}$ Scleromys;

- un diastème supérieur court (car. 4612), tandis qu'il est plus long chez Scleromys.

Dans l'AG1, S. angustus et $S$. quadrangulatus forment un clade alors que $S$. osbornianus diverge au nœud précédent. En effet, ce dernier développe: i) un mésolophe lingualement libre sur les P4 (car. 570) alors que ce dernier est connecté au postérolophe chez $S$. angustus et $S$. quadrangulatus; et ii) un bras postérieur du protoconide sur les p4 (car. 2690) qui est absent chez $S$. angustus et $S$. quadrangulatus. Scleromys praecursor diverge quant à lui bien plus tôt, et loin des autres représentants du genre, notamment en raison de l'épaisseur homogène de l'émail de l'hypoflexus et hypoflexide sur ses molaires (car. 130), alors qu' elle est hétérogène chez les trois autres espèces de Scleromys. Ces résultats suggèrent manifestement l'attribution de l'espèce $S$. praecursor à un nouveau genre. Nous révisons cette espèce dans la section Systématique et proposons de l'attribuer à Maquiamys praecursor n. comb.

D'après des analyses cladistiques récentes (Kramarz et al. 2013; Kerber et al. 2017, 2018; Rasia \& Candela 2017), Garridomys et Eoviscaccia sont soit des taxons apparentés au clade (Chinchillidae, Neoepiblemidae), soit plus étroitement apparentés à certains autres membres de ce clade. Cependant dans l'AG1, pas plus que pour Scleromys, ils ne sont inclus dans le groupe couronne des chinchilloïdes. En effet, ils ne partagent pas la majorité des caractères non-ambigus soutenant ce clade (nœud S8), ni celui des chinchillidés-néoépiblemidés (nœud S10):

- l'absence d'alignement entre le protolophe, la mure et le bras antérieur de l'hypocône sur les P4 (car. 630) et les M1-2 (car. 1980);

- l'absence de connection linguale du mésolophe sur les M3 (car. 1850); 
- l'absence d'alignement prononcé entre la mure et le bras antérieur de l'hypocône sur les M1-2 (car. 1960);

- la présence d'un métalophe sur les M1-2 (car. 2110);

- la présence de lamines sur des M1-2 fraîches (car. 2451) et sur les molaires inférieures (car. 4261);

- l'absence systématique de la néocristide dans l'antéroflexide des p4 (car. 2630);

- une échancrure où s'insère le tendon du masseter medialis pars infraorbitalis, située sous la m1 (car. 4401);

- une origine antérieure de la crête massétérique de la mandibule sous la m2 (car. 4421).

Les relations phylogénétiques des néoépiblemidés avec les autres groupes de chinchilloïdes ne sont pas claires. La plupart des études suggèrent une affinité avec les dinomyidés (e.g. Horovitz et al. 2006; Kramarz et al. 2013; Kerber et al. 2016) ou les chinchillidés (e.g. Kerber et al. 2017, 2018; Rasia \& Candela 2017). C'est cette dernière hypothèse de parenté entre les néoépiblemidés et les chinchillidés qui est ici soutenue dans l'AG1, sur la base de neuf caractères communs :

- l'absence d'alignement prononcé entre la mure et le bras antérieur de l'hypocône sur les P4 (car. 620), les M1-2 (car. 1960) et les M3 (car. 1970);

- l'absence d'alignement entre le protolophe, la mure et le bras antérieur de l'hypocône sur les P4 (car. 630) et les M1-2 (car. 1980);

- la présence de trois lamines sur des M1-2 fraîches ou peu usées (car. 2480);

- l'absence systématique de la néocristide dans l'antéroflexide des p4 (car. 2630);

- l'absence du néomésolophide sur les molaires inférieures (car. $\left.383^{\circ}\right)$;

- un foramen dans le canal lacrymal de taille moyenne (car. 4811).

Octodontoïdes: nouvelle définition des Acaremyidae et Adelphomyidae, n. stat., et taxons fossiles au sein du groupe couronne Contrairement à ceux de Verzi et al. (2014, 2016), les résultats d'Arnal \& Vucetich (2015a) et Arnal et al. (2017) soutiennent la divergence de plusieurs taxons fossiles en dehors du groupe couronne des octodontoïdes. Arnal \& Vucetich (2015a) reconnaissent ainsi cinq groupes basaux principaux:

- Draconomys (Argentine, fin Oligocène inférieur; Vucetich et al. 2010b);

- un groupe rassemblant Eosallamys (Pérou, ?Éocène supérieur/ Oligocène inférieur; Frailey \& Campbell 2004) et des taxons provenant de niveaux d'âge fin Oligocène inférieur-Oligocène supérieur: Paulacoutomys (Brésil; Vucetich et al. 1993b), Sallamys pascuali et Migraveramus (Bolivie; Hoffstetter \& Lavocat 1970; Lavocat 1976; Patterson \& Wood 1982), Llitun et Leucokephalos (Argentine; Vucetich et al. 2015b);

- un groupe rassemblant Eosachacui et Eoespina (Pérou, ?Éocène supérieur/Oligocène inférieur; Frailey \& Campbell 2004), et Sallamys? minutus (Brésil, fin Oligocène inférieur-Oligocène supérieur; Vucetich \& Ribeiro 2003);

- un groupe rassemblant divers taxons d'Argentine documentant une période temporelle étagée de la fin du Paléogène au Miocène moyen: Protacaremys, Acarechimys, Plesiacarechimys,
Caviocricetus, Dudumus, Platypittamys, Galileomys et Acaremys (Wood 1949; Vucetich et al. 1993a, 2010a, 2015b; Vucetich \& Verzi 1996; Walton 1997; Flynn et al. 2002, 2008; Vucetich \& Kramarz 2003; Kramarz 2004; Vucetich \& Vieytes 2006; Croft et al. 2011; Arnal et al. 2014; Tejada-Lara et al. 2015; Arnal \& Vucetich 2015b; Antoine et al. 2016);

- Prospaniomys (Vucetich et al. 2010a; Arnal \& Kramarz 2011).

Dans l'AG1, certains taxons sont situés basalement au sein des octodontoïdes, comme dans l'analyse d'Arnal \& Vucetich (2015a): Draconomys, Leucokephalos, Sallamys, Platypittamys, Galileomys, Acaremys, Prospaniomys, Plesiacarechimys, Caviocricetus, Dudumus et Prospaniomys. Cependant, ces taxons ne montrent pas la même séquence de branchement que dans l'analyse d'Arnal \& Vucetich (2015a), en raison:

- de la divergence plus précoce d'Eoespina-Eosachacui (voir section «Les groupes basaux des caviomorphes") et d'Eosallamys (voir section "Eosallamys et Mayomys») en dehors des octodontoïdes dans l'AG1;

- de la non-inclusion de certains taxons dans l'AG1: Migraveramus, Llitun, Paulacoutomys, Sallamys? minutus, Protacaremys et Acarechimys;

- de l'inclusion dans l'AG1 de taxons miocènes non pris en compte par Arnal \& Vucetich (2015a): Willidewu, Sciamys, Prostichomys et Pampamys (Vucetich \& Verzi 1991; Verzi et al. 1995; Kramarz 2001c, 2004; Flynn et al. 2002; Kramarz et al. 2010; Arnal \& Vucetich 2011; Olivares et al. 2012; Arnal \& Pérez 2013);

- du soutien d'une relation étroite entre Draconomys et Leucokephalos dans l'AG1. Ces deux taxons ont une largeur de la $\mathrm{m} 1$ supérieure à la celle de la p4 (car. 2530), tandis que les autres octodontoïdes montrent un état plus dérivé pour ce caractère (largeur de la $\mathrm{m} 1$ inférieure ou égale à celle de la p4; car. 2531);

- de la non-inclusion dans l'AG1 de Plesiacarechimys, Caviocricetus et Dudumus au sein du clade des acarémyidés (formés ici par Acaremys, Sciamys, Galileomys, Platypittamys, Deseadomys et Willidewu). Ces trois taxons présentent une rétention des prémolaires déciduales (car. 101), un caractère les rapprochant des adelphomyidés et du groupe couronne des octodontoïdes. De même, Caviocricetus et Dudumus sont caractérisés par une absence du bras postérieur du protoconide sur les m3 (car. 3741), comme chez la plupart des octodontoïdes modernes.

Arnal et al. (2017) reconnaissent un grand nombre de taxons basaux (11). Dans leur analyse, Caviocricetus et Dudumus ne divergent pas au sein des acarémyidés comme dans l'AG1. Les différences de composition des taxons basaux entre les analyses d'Arnal \& Vucetich (2015a) et d'Arnal et al. (2017) peuvent s'expliquer par un échantillonnage taxonomique différent, et l'ajout d'une vingtaine de nouveaux caractères dans Arnal et al. (2017) par rapport à Arnal \& Vucetich (2015a).

De plus, certains taxons (Adelphomys et Stichomys; Ameghino 1887; Kramarz 2004) qui appartiennent au groupe couronne des octodontö̈des dans les analyses d'Arnal \& Vucetich (2015a) et d'Arnal et al. (2017), occupent ici une position basale au sein de cette super-famille. Ils forment avec Prostichomys un clade qui peut être reconnu comme la famille des Adelphomyidae, n. stat. Patterson \& Pascual (1968) 
avaient initialement décrit, au sein des Echimyidae, la sous-famille des Adelphomyinae, qui regroupait des taxons mésodontes avec une forte tendance à l'obliquité des loph(id)es: Adelphomys, Stichomys, Paradelphomys, Xylechimys et Deseadomys. D'autres taxons ont été par la suite attribués à cette sous-famille comme Prostichomys, Microscleromys ou Quebradahondomys (Walton 1997; Kramarz 2001c; Croft et al. 2011). Cependant, Deseadomys semble être plus proche des acarémyidés que des adelphomyidés dans l'AG1 en raison de plusieurs caractères: i) partagés entre Deseadomys et les acarémyidés; et ii) non-partagés entre Deseadomys et les adelphomyidés :

- un remplacement des dp $4 / \mathrm{dP} 4 \mathrm{chez}$ Deseadomys (car. 100) au contraire d'une rétention chez les adelphomyidés;

- une largeur des M1-2 supérieure à leur longueur (car. $122^{0}$ et $123^{\circ}$ ) chez Deseadomys, tandis que leur largeur est égale ou inférieure à leur longueur chez les adelphomyidés;

- la formation d'une cristide postérieure formée par la fusion de l'hypolophide avec le postérolophide sur les p4 (car. 2881) comme chez les autres acarémyidés;

- la présence du bras postérieur du protoconide sur les m3 (car. $374^{\circ}$ ) chez Deseadomys, ce bras étant absent chez les adelphomyidés.

Concernant la position de Deseadomys, un résultat similaire est soutenu par l'analyse d'Arnal et al. (2017), dans laquelle ce taxon diverge au sein des octodontoïdes basaux, avant les acarémyidés, et apparaît très éloigné des adelphomyidés Adelphomys et Xylechimys.

Sciamys a été précédemment attribué aux acarémyidés (Arnal \& Vucetich 2011; Arnal \& Pérez 2013), ce qui est confirmé par l'AG1.

Willidewu et Pampamys ont historiquement été inclus au sein des échimyidés (Vucetich \& Verzi 1991; Verzi et al. 1995; Olivares et al. 2012; Olivares \& Verzi 2015), des relations non soutenues dans l'AG1:

- Willidewu diverge des échimyidés et se branche au sein des acarémyidés du fait de sa brachyodontie (car. 90), de la substitution de ses dp4/dP4 (car. 100) et d'un éperon du métaconide présent sur ses molaires inférieures (car. 3691); - Pampamys constitue l'un des octodontoïdes basaux qui divergent entre les acarémyidés et les adelphomyidés. Il est notamment caractérisé par un hypolophide des molaires inférieures peu oblique (car. $401^{\circ}$ ), tandis que chez la majorité des échimyidés cette cristide est fortement oblique. Il présente également une troisième cristide fortement orientée mésiolingualement sur les dp4 (car. 3282), alors que cette cristide est moins oblique ou droite chez les échimyidés.

Loretomys, restreint à CTA-32 et peu documenté (seulement connu par deux molaires supérieures), a été décrit comme un représentant des octodontoïdes, notamment en raison de sa brachyodontie, des lophes peu obliques et de l'absence du métalophe et de métacône sur ses molaires (Boivin et al. 2017b). Cette attribution est corroborée par l'AG9 dans laquelle Loretomys se place au sein des acarémyidés. Ils partagent avec certains membres de cette famille un bras antérieur de l'hypocône non aligné avec la mure sur les M1-3 (car. $196^{\circ}$ et 1970 ).
La structuration du groupe couronne des octodontoïdes de l'AG1 varie assez fortement de celles obtenues précédemment et s'appuyant sur des caractères uniquement morphologiques (Carvalho \& Salles 2004; Candela \& Rasia 2012; Olivares et al. 2012; Arnal \& Pérez 2013; Arnal et al. 2014, 2017; Arnal \& Vucetich 2015a, b). Ces différences observées entre l'AG1 et les études précédentes peuvent en partie s'expliquer par l'utilisation d'une contrainte pour les taxons modernes, fondées sur des données génétiques robustes (Upham \& Patterson 2015) dans l'AG1. Bien que Verzi et al. (2016) combinent à la fois caractères moléculaires et morphologiques pour leur analyse phylogénétique, les relations obtenues entre les échimyidés modernes diffèrent légèrement de celles de la contrainte utilisée dans l'AG1 (e.g. place de Thrichomys). Dans l'AG1, plusieurs taxons fossiles du Miocène-Pliocène se branchent au sein du groupe couronne des octodontoïdes : Pithanotomys, Xenodontomys, Neophanomys, Protadelphomys, Maruchito et Spaniomys (Vucetich \& Bond 1984; Reguero \& Candela 2001; Vucetich et al. 1992; Vucetich et al. 1993a; Vucetich \& Verzi 1994; Verzi 1999; Verzi et al. 1999, 2003, 2011; Flynn et al. 2002, 2008; Kramarz 2004; Deschamps \& Tomassini 2016).

Pithanotomys et Xenodontomys sont respectivement associés aux octodontidés et aux cténomyidés actuels dans l'AG1, un résultat en accord avec des études antérieures (Arnal \& Vucetich 2015a; octodontinés et cténomyinés sensu Verzi et al. 2016).

Neophanomys est généralement rapproché de l'ensemble octodontidés-cténomyidés (e.g. Verzi et al. 1999, 2011), une parenté soutenue dans l'étude phylogénétique de Verzi et al. (2016), Neophanomys se branchant au sein des octodontidés. Cependant, dans l'analyse d'Arnal \& Vucetich (2015a), Neophanomys est positionné en dehors de cette famille et des cténomyidés. Dans l'AG1, Neophanomys est associé aux cténomyidés, une relation soutenue par trois caractères nonambigus relatifs à la persistance des flexides (car. 3501, $413^{1}$ et 4231). Neophanomys diverge des octodontidés car il ne présente pas la majorité des caractères non-ambigus soutenant cette famille (car. $2261,392^{\circ}, 420^{3}, 421^{3}, 424^{1}$ et $425^{\circ}$ ).

Protadelphomys a été originellement décrit comme un échimyidé (Vucetich \& Bond 1984; Vucetich et al. 1992; Vucetich \& Verzi 1994). Protadelphomys a été ensuite placé en famille incertae sedis (Vucetich et al. 2010a; Antoine et al. 2016) et récemment rapproché des cténomyidés (Verzi et al. 2016). Dans l'AG1, Protadelphomys se branche au sein du clade formé par Euryzygomatomys et Mesocapromys/Mysateles car il partage avec ces taxons une obliquité des crêtes des molaires supérieures (car. 1961 $197^{1}$ et 1981) et une fissure sphénopalatine postéroventralement située par rapport au foramen lacrymal (car. 4871). Toutefois, Protadelphomys montre un remplacement de ses prémolaires déciduales (car. 10) à la différence des autres représentants du groupe couronne des octodontoïdes qui préservent les dents déciduales.

Maruchito est communément considéré comme un échimyidé (e.g. Vucetich et al. 1993a; Flynn et al. 2008), une relation de parenté retrouvée dans l'analyse de Verzi et al. (2016) où Maruchito se rapproche des Echimyini modernes Echimys et Makalata. Néanmoins, ici, bien que divergeant au sein des 
échimyinés, Maruchito est plus étroitement apparenté aux Myocastorini, notamment du fait de ses dp4 ayant un trigonide large (car. 3031) et un ectolophide distal (car. 3371), au contraire des Echimyini qui ne présentent pas ces caractères.

Bien que récemment rapporté aux abrocomidés (Verzi et al. 2016), Spaniomys est généralement attribué aux échimyidés (e.g. Flynn et al. 2002; Kramarz 2004). Dans l'AG1, Spaniomys est directement associé à Myocastor (comme dans l'analyse d'Arnal et al. [2017]) au sein des Echimyidae, Echimyinae et Echimyini. Son rattachement à cette tribu peut s'expliquer par certains caractères partagés avec les autres membres du groupe: - des crêtes légèrement obliques sur les molaires supérieures (car. 41);

- une M2 plus grande que la M1 (car. 1200);

- une échancrure où s'insère le tendon du masseter medialis pars infraorbitalis, située sous la $\mathrm{m} 1$ (car. $\left.440^{1}\right)$ et connectée aux crêtes massétérique et latérale (car. 4394).

Cephalomyidae: cavioïdes ou chinchilloïdes?

Cephalomys, Cephalomyopsis, Soriamys et Banderomys sont généralement regroupés au sein d'une même famille: les Cephalomyidae (Kramarz 2005). Ils documentent une fenêtre temporelle étagée de la fin de l'Oligocène inférieur au début du Miocène (Wood \& Patterson 1959; Vucetich 1985, 1989; Bond et al. 1998; Kramarz 2001a, 2005; Vucetich et al. 2010b, 2015b; Busker et al. 2017; Busker \& Dozo 2017), et sont caractérisés par des dents à couronne haute et avec des loph(-id)es qui fusionnent avec l'usure. Un autre taxon, Litodontomys, découvert à Cabeza Blanca en Argentine (fin de l'Oligocène inférieur-Oligocène supérieur; Loomis 1914; Wood \& Patterson 1959) et qui semble développer ces mêmes caractères, a parfois été rapproché de cette famille (e.g. Simpson 1945; Kramarz 2001a, 2005). Les céphalomyidés possèdent une histoire systématique complexe. Ils ont été tout d'abord considérés comme un stock basal des hystricomorphes (Ameghino 1897), puis ont été tour à tour attribués à trois des quatre super-familles: aux octodontoïdes (Miller \& Gidley 1918), aux cavioïdes (Simpson 1945; McKenna \& Bell 1997) et aux chinchilloïdes (Loomis 1914; Wood 1955; Landry 1957). La validité des céphalomyidés a également été remise en question par Wood \& Patterson (1959) et Patterson \& Wood (1982). Par la suite, la découverte de restes crâniens et dentaires de céphalomyidés, datés de la fin du Paléogène et du Miocène ont permis d'accroître les connaissances sur la diversité et l'évolution du groupe (Kramarz 2005). Les études effectuées sur ce matériel ont appuyé l'hypothèse d'une affiliation de cette famille aux chinchilloïdes (Vucetich 1985, 1989; Dozo 1995; Vucetich et al. 1999; Kramarz 2001a). Cependant, Kramarz (2001a) a noté de fortes ressemblances ostéologiques et dentaires entre les céphalomyidés et certains cavioïdes (caviidés et «éocardiidés»). À l'occasion de l'étude dans laquelle il décrit le genre Banderomys, Kramarz (2005) a réalisé une analyse cladistique incluant Cephalomys, Soriamys, Banderomys et des représentants de chacune des super-familles auxquelles ont été précédemment rapportés les céphalomyidés: les octodontoïdes Platypittamys, Proechimys, Abrocoma et Octodontomys; le cavioïde Luantus; et les chinchilloïdes Perimys et la combinaison (Lagostomus + Eoviscaccia). Les résultats de cette analyse corroboraient la monophylie des trois céphalomyidés et une étroite parenté de cette famille avec le cavioïde Luantus plutôt qu'avec les chinchilloïdes. Cette relation Cephalomyidae-Cavioidea a été récemment soutenue par l'analyse cladistique de Busker \& Dozo (2018). Selon les résultats de ces auteurs, Litodontomys serait inclus au sein des céphalomyidés au contraire du genre Cephalomyopsis qui présenterait une plus étroite parenté avec les autres cavioïdes de l'analyse. Le genre Cephalomys réunissait traditionnellement trois espèces d'Argentine, $C$. arcidens (espèce type), C. plexus et C. ceciae (Cabeza Blanca et/ou La Flecha; Wood \& Patterson 1959), et une de Bolivie, C. bolivianus. Cette dernière a originellement été décrite par Lavocat (1976) sur la base d'un seul fragment de crâne provenant de Salla (fin de l'Oligocène inférieur-Oligocène supérieur) et portant des P4-M2 très usées. Dans les années 1980 et 1990, plusieurs spécimens non publiés montrant différents stades d'usure dentaire ont été découverts et semblent documenter le même taxon décrit par Lavocat. Cependant, ce taxon se différencie des autres espèces de Cephalomys par plusieurs caractères dentaires et apparaîtrait plus proche de l'espèce Asteromys punctus (Pérez et al. 2018). Ce taxon a ici été inclus dans la matrice sous le nom "Asteromys» bolivianus.

Des membres des céphalomyidés (Cephalomys arcidens et Soriamys) ou des taxons rapprochés de cette famille ( $«$ Asteromys» bolivianus et Litodontomys) ont été ici intégrés à plusieurs analyses (AG3-AG7; Tableau 2). Les résultats obtenus en AG3 et AG6, analyses pour lesquelles la position de ces taxons et celle d'Asteromys punctus n'ont pas été contraintes, soutiennent: - l'inclusion de Litodontomys au sein des cépalomyidés (AG3 et AG6) qui est notamment soutenue par deux NA: la forme ovale des lobes antérieur (car. 4203) et postérieur (car. 4213) des m1-2;

- l'inclusion d' "Asteromys» bolivianus au sein des céphalomyidés (AG3 et AG6);

- une relation de parenté plus étroite entre "Asteromys» bolivianus et $C$. arcidens plutôt qu'avec $A$. punctus (AG3 et AG6). Cette association est soutenue par deux NA: la présence d'un sillon labial opposé à l'hypoflexus sur des dents supérieures à un stade d'usure avancé (car. $6^{1}$ ) et une $\mathrm{m} 2$ plus longue que la m3 (car. 3542). Ces résultats laissent envisager une appartenance d' "Asteromys» bolivianus (voire de Litodontomys) au genre Cephalomys. Asteromys punctus est soit inclus au sein des céphalomyidés (AG3), soit correspond à un cavioïde divergeant avant l'émergence de cette famille (AG6). Cependant, ce taxon n'étant documenté que par des dents inférieures, il ne présente qu'un état réellement différent sur les deux NA soutenant l'affiliation d' "Asteromys" bolivianus et de C.arcidens: $354^{1}$ (le caractère 6 est codé «?" chez $A$. punctus);

- la non inclusion de Soriamys aux céphalomyidés (AG3).

Néanmoins, la position des taxons reconnus comme des céphalomyidés par ces analyses varient entre l'AG3 (avec Soriamys) et l'AG6 (sans Soriamys) : ils divergent soit au sein des chinchilloïdes (AG3), soit au sein des cavioïdes (AG6). Au vu de ces résultats contradictoires, plusieurs analyses pour lesquelles la position de ces taxons est contrainte dans l'une 
ou l'autre des deux super-familles ont été réalisées (AG4, AG5 et AG7; Tableau 2). Le nombre de pas entre l'AG3 versus les AG4/AG 5 varie de manière plus importante $(3,49$ et 4,16 pas supplémentaires) que les différences respectives entre l'AG4 et l'AG5 (0,67 pas d'écart) et l'AG6 et l'AG7 (0,34 pas d'écart), révélant ainsi une forte instabilité du groupe (Tableau 2). Ainsi, il apparaît difficile de statuer quant à l'appartenance des céphalomyidés à une super-famille donnée. De plus, ces résultats sont également à prendre avec précaution du fait de la position incertaine de Perimys au sein des Caviidae (voir section "Les cavioïdes basaux et le cas de Perimys») et nécessiteraient d'être confirmés pour les autres membres des Cephalomyidae (C. plexus, C. ceciae, Cephalomyopsis et Banderomys).

RÉSULTATS PHYLOGÉNÉTIQUES ET DONNÉES STRATIGRAPHIQUES L'arbre de consensus strict de l'AG1 (Fig. 8) replacé dans un contexte stratigraphique est particulièrement éclairant, dans la mesure où il révèle et souligne la présence de plusieurs lignées-fantômes depuis la fin de l'Éocène moyen.

Les extensions fantômes se localisent essentiellement au niveau des représentants modernes de chacune des quatre super-familles de caviomorphes. Ces lignées-fantômes s'étendent généralement du Miocène (Miocène inférieur, moyen ou supérieur) jusqu'à nos jours. Elles correspondent sans doute pour la plupart à des extensions artificielles, inhérentes à l'échantillonnage taxonomique de l'analyse, qui n'inclut aucun taxon quaternaire, presque aucun taxon pliocène et une partie seulement des taxons miocènes connus (par opposition à l'échantillonnage quasi-exhaustif des caviomorphes paléogènes). Toutefois, certaines lignées-fantômes sont potentiellement révélatrices de lacunes du registre fossile $(\mathrm{du}$ moins en partie). Deux exemples, l'un chez les octodontoïdes et l'autre chez les éréthizontoïdes sont ci-dessous développés.

La lignée-fantôme de l'octodontoïde Abrocoma s'étend ici du début du Miocène inférieur à nos jours. Abrocoma et Cuscomys (un autre taxon actuel non considéré dans l'analyse) sont réunis au sein de la famille des abrocomidés, à laquelle est rattaché un seul genre fossile, non intégré dans l'AG1: Protabrocoma (Miocène supérieur). Récemment, Verzi et al. (2016) ont inclus le genre Spaniomys (Miocène inférieur) au sein de cette famille mais ce dernier apparaît ici plus proche phylogénétiquement de Myocastor. En considérant Protabrocoma comme étroitement apparenté à Abrocoma et les résultats obtenus dans l'AG1, les abrocomidés comprendraient alors une lignée-fantôme couvrant la majeure partie du Miocène.

La majorité des éréthizontoïdes ont été inclus dans l'AG1 : les trois genres actuels (Coendou, Erethizon et Chaetomys) et tous les genres fossiles à l'exception d'Eopululo (?Éocène supérieur/Oligocène inférieur), Parasteiromys (Miocène inférieur), Microsteiromys (fin Miocène moyen) et Paradoxomys (fin Miocène suprérieur). Ces quatre derniers taxons ne semblent pas avoir d'affinité directe avec Chaetomys, au patron dentaire très particulier, et ils se branchent probablement au sein des éréthizontinés (représentés par les deux autres genres actuels). Ainsi, l'extension fantôme de Chaetomys du Miocène inférieur à nos jours témoignerait bien d'une lacune manifeste du registre fossile.
Le cas de la très longue lignée-fantôme du clade (Dasyprocta, Cuniculus), qui s'étend de l'Oligocène inférieur à l'époque actuelle, est particulier. Plusieurs taxons éteints, précédemment attribués aux dasyproctidés, ont été inclus dans les analyses: Eobranisamys, Eoincamys, Microscleromys, Branisamys, Australoprocta et Neoreomys. Cependant, les résultats obtenus dans l'AG1 remettent clairement en question leur assignation à cette famille (voir section "Les cavioïdes basaux et le cas de Perimys»). Toutefois, plusieurs dasyproctidés présumés n'ont pas été inclus au sein des analyses: Eopicure (?Éocène supérieur/Oligocène inférieur; Frailey \& Campbell 2004), Andemys (Oligocène inférieur; Bertrand et al. 2012), Allloiomys (Miocène moyen; Vucetich 1984), Megastus (Miocène moyen; Vucetich 1984), Mesoprocta (fin du Miocène moyen; Croft et al. 2011) et Plesiaguti (Pléistocène; Vucetich \& Verzi 2002). Ainsi, en l'état actuel, il apparaît difficile de conclure si la lignée-fantôme du clade (Dasyprocta, Cuniculus) est seulement représentative d'un registre fossile lacunaire ou non.

Deux taxons, Pampamys et Microscleromys, présentent de très longues extensions fantômes (de plus de 14 et $15 \mathrm{Ma}$, respectivement) qui pourraient témoigner d'importants hiatus dans le registre fossile. Cependant, concernant Pampamys, sa position basale au sein des octodontoïdes, obtenue dans l'AG1, est en contradiction avec des analyses précédentes (Olivares et al. 2012; Verzi et al. 2014, 2016; Olivares \& Verzi 2015), dans lesquelles ce taxon apparaît plus étroitement apparenté au genre actuel Thrichomys et au genre éteint Eumysops (Argentine; Pléistocène-Pliocène) qu'aux octodontoïdes basaux. La position de Pampamys dans l'AG1 repose essentiellement sur deux caractères qu'il ne partage pas avec la majorité des taxons du groupe couronne des octodontoïdes (voir section "Octodontoïdes: nouvelle définition des Acaremyidae et Adelphomyidae, n. stat., et taxons fossiles au sein du groupe couronne»); elle nécessiterait d'être confirmée par de plus amples analyses incluant Eumysops.

Plusieurs lignées fantômes anciennes apparaissent à des périodes clefs dans l'histoire évolutive des caviomorphes: au niveau de la divergence du groupe couronne des caviomorphes (Éocène moyen-Éocène supérieur), de la mise en place des super-familles (Éocène supérieur-Oligocène inférieur) et au sein de chacune des super-familles (notamment au niveau de leurs taxons basaux; Oligocène, voire Miocène inférieur pour les chinchilloïdes et les éréthizontoïdes). Ces lignées-fantômes sont probablement révélatrices de lacunes dans les archives paléontologiques. En effet, bien que le registre fossile se soit considérablement enrichi dans la dernière décennie (Shockey et al. 2009; Vucetich et al. 2010b, 2014, 2015b; Cerdeño 2011 ; Bertrand et al. 2012; Pérez et al. 2012; Antoine et al. 2012, 2016, 2017; Boivin et al. 2017a, b, 2018a; Kerber et al. 2017), le registre paléogène des caviomorphes demeure encore très sporadique et ce, plus particulièrement, de l'Éocène moyen à l'Oligocène inférieur.

Enfin, à l'inverse, certaines lignées-fantômes sont probablement sous-estimées à partir des résultats de l'AG1, en raison de la non-prise en compte de taxons paléogènes peu documentés comme Eopululo et Eodelphomys (Santa Rosa, Pérou, ?Éocène supérieur/Oligocène inférieur; Frailey \& Campbell 
2004). Eopululo est le plus ancien éréthizontoïde présumé, et l'octodontoïde Eodelphomys pourrait être le plus ancien représentant des adelphomyidés. Ainsi, les âges d'apparition des éréthizontoïdes et des différents octodontoïdes basaux sont potentiellement plus anciens que ceux déduits des résultats de l'AG1, replacés dans leur contexte stratigraphique.

\section{MORPHOLOGIE DENTAIRE DES CAVIOMORPHES Évolution morphologique du patron dentaire des caviomorphes au Paléogène}

Comme mentionné auparavant (voir section «Les groupes basaux des caviomorphes»), le plan dentaire ancestral des caviomorphes était selon toute vraisemblance proche de ceux de Cachiyacuy et de Canaanimys, c'est-à-dire caractérisé par: - des dents brachyodontes, non-taeniodontes, avec des loph(-id)es fins et peu obliques;

- des dents supérieures pentalophodontes avec un fort métalophe montrant lingualement des connexions avec la troisième crête ou à l'extrémité mésiale du bras antérieur de l'hypocône (et potentiellement avec le postérolophe);

- des molaires inférieures probablement tétralophodontes avec une deuxième cristide complète (Boivin 2017; Boivin \& Marivaux 2018);

- des dp4 probablement pentalophodontes avec le développement d'un bras postérieur au protoconide, mésolophide et néomésolophide (Boivin 2017; Boivin \& Marivaux 2018).

Comme en témoignent les taxons des localités de la fin de l'Éocène moyen de Contamana, ce patron s'est rapidement modifié avec l'apparition de certains caractères: la taeniodontie (e.g. Canaanimys, Pozomys et Eobranisamys javierpradoi), un métalophe fortement connecté lingualement au postérolophe et perdant sa connexion avec la troisième crête (e.g. Eobranisamys), et un métalophe réduit, tendant même à disparaître (e.g. Cachiyacuy, Canaanimys, Pozomys et cf. Eoespina sp. de CTA-27).

À l'Éocène supérieur-Oligocène inférieur, la mise en place et la diversification basale des quatre super-familles sont probablement allées de pair avec l'accentuation des divergences morphologiques et l'acquisition de traits de spécialisation à l'intérieur de chaque super-famille. Les loph(-id)es tendent à devenir obliques, la deuxième cristide transverse et le métalophe se réduisent (voire disparaissent) respectivement sur les molaires inférieures et supérieures et la hauteur de la couronne s'élève (i.e., mésodontie ou protohypsodontie) chez plusieurs taxons dont Tarapotomys, les premiers chinchilloïdes (e.g. Eoincamys) et des adelphomyidés présumés (e.g. Eodelphomys et Adelphomyinae indet. de TAR-13; Frailey \& Campbell 2004). Ainsi ces caractères apparaîtraient de façon convergente entre certains caviomorphes, chinchilloïdes et octodontoïdes basaux. Chez les chinchilloïdes, l'obliquité des loph(-id)es est maximale et s'accompagne d'une troisième crête transverse réduite à un court mésolophe qui se connecte lingualement au postérolophe (e.g. Eoincamys pascuali et Eoincamys parvus; Frailey \& Campbell 2004). Dès l'Oligocène inférieur, certains chinchilloïdes (Eoviscaccia frassinettii, Chinchilloidea indet. de TAR-01 et Chinchilloidea? gen. et sp. 2 de La Cantera; Vucetich et al. 2010b; Bertrand et al.
2012; Boivin et al. 2018a) présentent des patrons dentaires plus dérivés, caractérisés par une couronne plus haute, la formation de lobes/lamines et une hétérogénéité de l'épaisseur de l'émail importante entre les bords d'attaque et les bords de fuite des dents jugales (voir Kramarz et al. 2013). La réduction ou l'absence de métalophe sur les molaires supérieures est également observée chez les octodontoïdes (ou formes apparentées; voir sections "Eosallamys et Mayomys", et "Octodontoïdes: nouvelle définition des Acaremyidae et Adelphomyidae, n. stat., et taxons fossiles au sein du groupe couronne", etc.) Mayomys, Draconomys, Selvamys (Boivin et al. 2018a) et Vallehermosomys (Vucetich et al. 2010b). Chez ces taxons, les loph(-id)es sont peu obliques, les néomésolophide et mésostylide tendent à disparaître et le bras postérieur du protoconide tend à se réduire sur les molaires inférieures. À l'Éocène supérieur-Oligocène inférieur, seraient enregistrés les plus anciens éréthizontoïdes présumés avec Eopululo, Kishkasteiromys et Shapajamys (Frailey \& Campbell 2004; Boivin et al. 2018a; voir section "Les groupes basaux des caviomorphes" pour le cas de Shapajamys). Kishkasteiromys et Shapajamys ont des molaires supérieures avec un métalophe connecté au postérolophe, à la différence d'Eopululo chez lequel aucun métalophe n'est développé. Les molaires d'Eopululo et Shapajamys présentent des flexi(-des) étendus. Enfin, un assez large panel de tailles semble présent à cette époque, allant de très petites formes (e.g. Selvamys; $\mathrm{ML}=c \cdot 1,20$ et $\mathrm{Ml}=c .1,37$ ) à des taxons de taille moyenne (e.g. Caviomorpha indet. 2. de TAR-22; $\mathrm{ML}=3,73$ et $\mathrm{Ml}=3,77$; Boivin et al. 2018a).

Les caractères auparavant observés chez les éréthizontoïdes, chinchilloïdes et octodontoïdes pré-déséadiens se retrouvent respectivement chez des membres de chacune de ces super-familles au Déséadien (ainsi qu'au Miocène) : Protosteiromys pour les éréthizontoïdes; Chambiramys, Maquiamys n. gen., Incamys et Eoviscaccia boliviana pour les chinchilloïdes (Lavocat 1976; Patterson \& Wood 1982; Vucetich 1989; Boivin et al. 2017b; voir section "Systématique»); et par exemple Loretomys, Platypittamys, Sallamys et Galileomys pour les octodontoïdes (Wood 1949; Lavocat 1976; Patterson \& Wood 1982; Vucetich et al. 2015b; Boivin et al. 2017b). Certaines de ces tendances s'accentuent même chez des représentants des chinchilloïdes (dont Chambiramys) et des octodontoïdes (e.g. Platypittamys, Sallamys et Galileomys). Chez Chambiramys, la deuxième cristide transverse des molaires inférieures et la troisième crête transverse des molaires supérieures peuvent être très réduites ou même absentes. Certains octodontoïdes déséadiens comme Platypittamys, Deseadomys et Galileomys montrent un patron dentaire proche de celui de Mayomys, Draconomys, Selvamys et Vallehermosomys, mais avec toutefois un mésostyle plus développé et généralement situé plus distalement sur les molaires supérieures. Outre l'absence de néomésolophide et de mésostylide, ces octodontoïdes déséadiens ont également un bras postérieur du métaconide réduit ou absent sur les molaires inférieures. Le Déséadien est caractérisé par l'apparition non-ambiguë dans le registre fossile des caviidés, avec les genres Asteromys et Chubutomys 
(Wood \& Patterson 1959; Pérez \& Vucetich 2012b; Pérez et al. 2012). Ces taxons ont des dents à couronne haute (mésodontes ou hypsodontes) sur lesquelles les loph(-id) es fusionnent entre eux avec l'usure et forment deux lobes séparés par un hypoflexus/ide transversalement étendu. Les molaires inférieures d'Asteromys présentent un plan clairement tétralophodonte, développant quatre longues cristides transverses sur germes dentaires et dents fraîches. Chubutomys est caractérisé par des molaires inférieures où la fusion des lophides semble s'initier rapidement. Aucun germe dentaire n'étant attribué à ce taxon (Wood \& Patterson 1959; Pérez et al. 2010, 2012), le nombre de cristides sur les molaires inférieures de Chubutomys ne peut toutefois être affirmé de façon certaine.

\section{Caractéristiques dentaires et tendances évolutives chez les quatre super-familles}

La définition de caractères dentaires propres à chacune des super-familles de caviomorphes pouvant être applicables à tous leurs représentants est quasiment impossible pour plusieurs raisons:

- de nombreux traits ont une évolution convergente et se retrouvent au sein de plusieurs super-familles et/ou caviomorphes basaux de manière indépendante. Par exemple, une augmentation de la hauteur de la couronne à partir d'une condition brachyodonte est typique des cavioïdes, des chinchilloïdes et du groupe couronne des octodontoïdes, mais se développe également chez certains octodontoïdes (e.g. Sallamys) et caviomorphes basaux (e.g. Tarapotomys) et tend à se développer chez l'éréthizontoïde Hypsosteiromys (Candela \& Vucetich 2002);

- en plus de $40 \mathrm{Ma}$ d'évolution, la plupart des patrons dentaires ont été modifiés, parfois de façon considérable (e.g. le cas des Hydrochoerinés chez les cavioïdes);

- les caractères dentaires se mettent généralement en place de façon progressive (transformations additives). C'est le cas par exemple de l'élévation de la couronne dentaire chez les caviidés (Pérez \& Vucetich 2011, 2012a);

- les taxons montrent souvent une mosaïque de caractères témoignant: i) de différences marquées en matière de temps d'apparition et de tempo évolutif de ces traits; et ii) de probables évolutions parallèles entre taxons proches phylogénétiquement. Par exemple, les molaires supérieures d'Eoincamys parvus paraissent être plus dérivées que celles d'Incamys bolivianus, du fait de l'absence totale de métalophe et d'une connexion toujours linguale entre mésolophe et postérolophe chez le premier. Chez I. bolivianus, ces caractères sont en effet plus variables: le métalophe est présent ou absent et le mésolophe est libre ou lié au bras antérieur de l'hypocône ou au postérolophe. En revanche, I. bolivianus paraît plus dérivé de par une épaisseur hétérogène de l'émail entre les bords d'attaque et de fuite sur ses dents (Vucetich et al. 2015b), au contraire d'E. parvus.

Quoi qu'il en soit, les super-familles peuvent tout de même être définies sur la base des synapomorphies présentes aux nouds correspondants dans l'AG1 et distinguées en outre par certaines tendances ou associations de caractères, qui seront syntéthisées ci-dessous.
Les Erethizontoidea. Les éréthizontoïdes présentent des dents brachyodontes, généralement non-taeniodontes, et avec des loph(-id)es peu obliques. Les flexi(-des) labiaux sur les molaires supérieures et linguaux sur les molaires inférieures peuvent être étendus. Les dents supérieures montrent généralement une mure complète, un métacône et un métalophe fort ou réduit. Des $\mathrm{P} 4$ sont molarisées avec une courte extension postérieure de l'hypocône ne rejoignant pas l'hypocône. Certains taxons ont un mésostyle et métacône qui tendent à se fusionner en une large cuspide sur les P4 et M1-3 (car. 48 et 210 en Annexe 2): Eosteiromys, Steiromys, Coendou et Erethizon. Les dp4 et p4 possèdent un trigonide large. Les dp4 peuvent devenir hexalophodontes et les $\mathrm{p} 4$ et molaires inférieures pentalophodontes, suite au développement de loph(-id)es annexes (voir Boivin 2017; Boivin \& Marivaux 2018). Les dp4 possèdent généralement des deuxième et troisième cristides bien développées. Les $\mathrm{p} 4$ et molaires inférieures ont une deuxième cristide le plus souvent complète et bien développée.

Les Cavioidea. Les cavioïdes présentent des dents mésodontes ou hypsodontes (protohypsodontes et euhypsodontes), nontaeniodontes ou taeniodontes et avec des loph(-id)es peu obliques ou obliques. Les P4 sont molarisées ou non, avec généralement une forte réduction ou l'absence de la troisième crête et du métalophe. Les molaires supérieures sont le plus souvent pentalophodontes mais avec une troisième crête et un métalophe parfois réduits. Les $\mathrm{p} 4$ peuvent être caractérisées par l'absence de métalophulide I (e.g. Asteromys). Les molaires inférieures sont habituellement tétralophodontes mais avec une deuxième cristide parfois réduite. Certains cavioïdes sont caractérisés par la présence, voire l'abondance de cément. Chez les caviidés, les loph(-id)es fusionnent entre eux avec l'usure et forment deux lobes séparés par un hypoflexus/ide transversalement étendu et la présence habituelle d'une projection postérieure du lobe distal sur les M3. Chez certains membres de cette famille, le patron dentaire peut se modifier suite à la formation de nouveaux flexi(-des) (e.g. Cavia, hydrochoerinés) et l'ajout de lamines (chez les hydrochoerinés).

Les Chinchilloidea. Chez les chinchilloïdes, les dents sont généralement mésodontes ou hypsodontes (protohypsodontes et euhypsodontes), taeniodontes et avec des loph(-id)es très obliques. Les dents supérieures, souvent dépourvues de métalophe, présentent une troisième crête réduite à un court mésolophe libre ou connecté au postérolophe. Les dp4 se caractérisent par la réduction de la troisième cristide à un néomésolophide connecté à la deuxième cristide ou par l'absence de la troisième cristide. Les $\mathrm{p} 4$ et les molaires inférieures ont une deuxième cristide très réduite, voire absente. Quand elle est présente, cette cristide apparait être un court néomésolophide libre ou connecté au métalophulide I. Certains chinchilloïdes peuvent être caractérisés par la présence, voire l'abondance de cément, une épaisseur hétérogène de l'émail entre les bords d'attaque et de fuite sur ses dents, la formation de lamines et/ ou de lobes avec l'usure (par fusion des loph(-id)es, et l'ajout de lamines/loph(-id)es surnuméraires. 
60

50

40

30

20

\begin{tabular}{|c|c|c|c|c|c|c|c|c|c|c|c|c|c|c|c|c|c|}
\hline \multirow{2}{*}{\multicolumn{3}{|c|}{$\begin{array}{l}\text { PALÉOCÈNE } \\
\text { inférieur|moy.sup. }\end{array}$}} & \multicolumn{4}{|c|}{ ÉOCÈNE } & \multicolumn{2}{|c|}{ OLIGOCÈNE } & \multicolumn{6}{|c|}{ MIOCÈNE } & \multicolumn{2}{|c|}{ PLIO. pLÉISTO. } & \multirow{2}{*}{ Époque } \\
\hline & & & inférieur & moye & & sup. & inférieur & sup. & & ieur & $\mathrm{mo}$ & en & supér & & & & \\
\hline Danien & $\begin{array}{l}\frac{\bar{d}}{\Phi} \\
\frac{0}{0} \\
\frac{\mathbb{\sigma}}{\mathbb{D}} \\
\omega\end{array}$ & 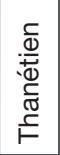 & Yprésien & Lutétien & 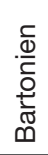 & 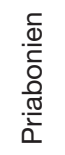 & Rupélien & 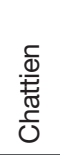 & 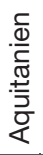 & 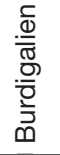 & 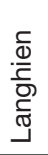 & 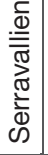 & 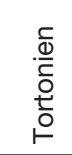 & 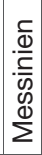 & 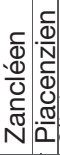 & 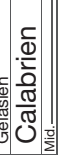 & Stage \\
\hline
\end{tabular}

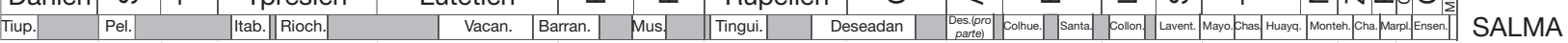

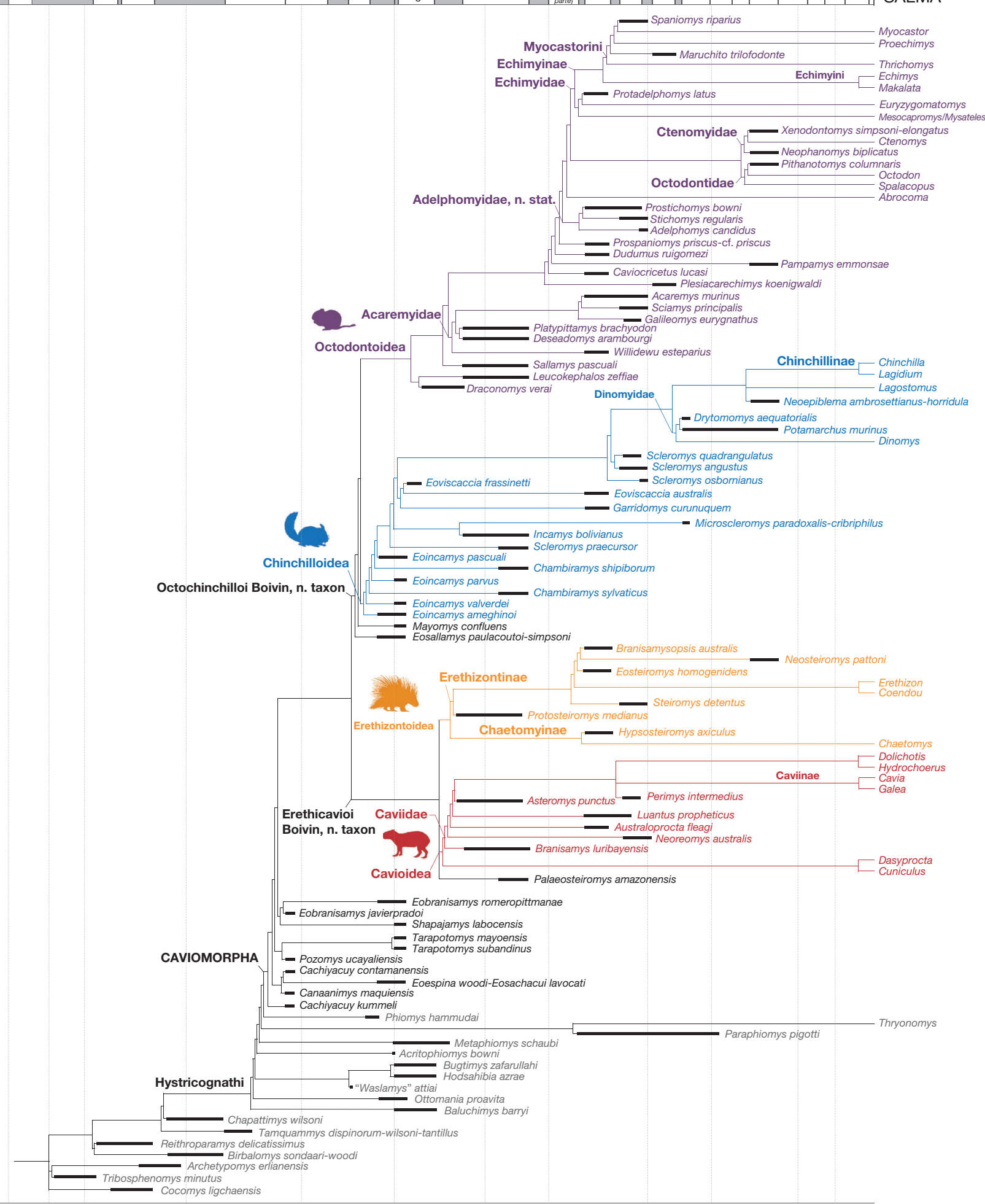

FIG. 8. - Résultats phylogénétiques de l'AG1 (Fig. 5), replacés dans un contexte stratigraphique. Le code couleur est le même que celui de la Figure 3. 
Les Octodontoidea. Chez les octodontoïdes, les dents sont brachyodontes, mésodontes ou hypsodontes (protohypsodontes et euhypsodontes), généralement non-taeniodontes et avec des loph(-id)es peu obliques ou obliques. Les dents supérieures n'ont généralement plus de métalophe et ont tendance à perdre le métacône (ces deux structures peuvant être indistinctes, noyés dans le postérolophe). Les dents supérieures possèdent une large cuspide labiodistale qui correspondrait à un mésostyle distalement déplacé et développé en surface et hauteur (ou à une structure résultant de la fusion du mésostyle et du métacône; voir Boivin 2017; Boivin et al. 2018a; Boivin \& Marivaux 2018). Les dp 4 sont caractérisées par des deuxième et troisième cristides complètes, réduites ou absentes. Chez de nombreux octodontoïdes, il est à noter la rétention des dP4/dp4. Chez certains taxons (Platypittamys, Deseadomys, Galileomys, Acaremys et Sciamys), l'hypolophide, l'entoconide et le postérolophide fusionnent en une seule cristide épaisse sur les p4. Dans ce groupe, on peut noter également l'absence fréquente du néomésolophide et du mésostylide sur les $\mathrm{p} 4$ et les molaires inférieures, la réduction des bras postérieurs du métaconide et du protoconide, et la présence de crêtes annexes (dont la cristide du métaconide; voir Boivin 2017; Boivin \& Marivaux 2018) participant à la formation de la deuxième cristide. Chez les abrocomidés, octodontidés, cténomyidés et certains acarémyidés, on peut noter le développement d'un ou de plusieurs lobes avec l'usure. Dans de rares cas, on observe la formation de lamines et un développement important de cément.

\section{APPARITION DES GRANDS GROUPES \\ ET RADiations AU PALÉOGÈnE ET AU Miocène \\ Âges de divergence des principaux clades}

Les résultats de l'AG1, une fois replacés dans un contexte stratigraphique, fournissent des hypothèses quant aux âges de divergence des clades (Fig. 8 ; Tableau 4). Les caviomorphes et le groupe couronne des caviomorphes divergeraient pendant le Vacien (i.e., Éocène moyen), peu avant l'enregistrement des plus anciens représentants du groupe dans les localités éocènes de Contamana (Barrancien, fin Éocène moyen). Les quatre super-familles se mettraient en place de façon asynchrone : au Mustersien (i.e., fin Éocène moyen-début Éocène supérieur) pour les chinchilloïdes et octodontoïdes, et au Tinguiriricien (i.e., Oligocène inférieur) pour les éréthizontoïdes et cavioïdes. Cependant, les âges d'apparition des chinchilloïdes et des octodontoïdes sont ambigus en raison de l'âge incertain de la faune de Santa Rosa (?Éocène supérieur/Oligocène inférieur; Frailey \& Campbell 2004). Si un âge Oligocène inférieur est considéré pour cette faune d'après certains auteurs (Shockey et al. 2004; Croft et al. 2008; Vucetich et al. 2010b, 2015b; Antoine et al. 2012, 2017; Kay 2015; mais voir Goin \& Candela 2004; Bond et al. 2015), l'apparition de ces deux super-familles, potentiellement plus récente, remonterait à la toute fin de l'Éocène supérieur. De plus, comme précédemment évoqué (voir section "Résultats phylogénétiques et données stratigraphiques»), les âges de divergence des éréthizontoïdes et des cavioïdes sont potentiellement antérieurs, car des représentants anciens présumés de ces super-familles, Eopululo pour les éréthizontoïdes et Eopicure pour les cavioïdes (tous deux découverts à Santa Rosa) n'ont pas été inclus dans l'AG1. Si Eopululo se branche au sein des éréthizontoïdes et/ou Eopicure parmi les cavioïdes, la divergence entre les éréthizontoïdes et les cavioïdes serait alors fin Mustersien (début de l'Éocène supérieur, c. $36 \mathrm{Ma}$ ), un âge équivalant à la divergence chinchilloïdes-octodontoïdes. En outre, l'absence de taxons basaux reconnus pour les éréthizontoïdes et les cavioïdes, suggère soit une origine plus ancienne des deux super-familles (et de fait une incomplétude du registre fossile), soit une mise en place très rapide des (super-)familles actuelles. Selon les résultats de l'AG1 et en considérant un registre fossile non-biaisé, les Erethizontinae, les Chaetomyinae, les Caviidae, le clade regroupant les Dasyproctidae et Cuniculidae, et les Acaremyidae divergeraient tous à l'Oligocène inférieur, autour de $30 \mathrm{Ma}$. La majorité des grands groupes restants se mettrait en place au Miocène inférieur: Caviinae, Hydrochoerinae, Dolichotinae, Abrocomidae, Echimyidae, Capromyinae, Euryzygomatomyinae, Echimyinae, Echimyini, Myocastorini et Adelphomyidae, n. stat. Toutefois, l'âge d'apparition de cette dernière famille est potentiellement antérieur car des taxons, qui pourraient être ses plus anciens représentants, Eodelphomys (Santa Rosa) et Adelphomyinae, gen. et sp. indet. de TAR-13 (Tarapoto/ Shapaja, Oligocène inférieur; Boivin et al. 2018a), n'ont pas été pris en compte dans les analyses (voir section "Choix des taxons»). D'après l'AG1, le groupe couronne des Chinchilloidea et les Dinomyidae apparaîtraient au Miocène moyen, et les Octodontidae, les Ctenomyidae, les Neoepiblemidae et les Chinchillidae au Miocène supérieur. Certains représentants néogènes et quaternaires de ces groupes n'ont toutefois pas été inclus dans les présentes analyses, et il semble évident que ces âges de divergence sont particulièrement sous-estimés (par exemple, les chinchillidés lagostominés sont sans le moindre doute connus dès le Miocène inférieur avec Prolagostomus et Pliolagostomus; Vucetich 1984; Flynn et al. 2002, 2008).

Les âges de divergence des groupes couronnes des superfamilles, des familles et sous-familles de l'AG1 different généralement de ceux obtenus lors de précédentes études fondées (au moins en partie) sur des caractères morphologiques et focalisées sur une partie seulement des caviomorphes (Pérez \& Pol 2012; Arnal \& Vucetich 2015a; Verzi et al. 2016; Kerber et al. 2018; Tableau 4) pour trois raisons principales:

- un échantillonnage taxonomique différent entre les analyses: ces études incluent des taxons non pris en compte dans l'AG1. Ils peuvent documenter et ainsi réduire les lignées-fantômes obtenues dans l'AG1 (e.g. Phanomys, Guiomys ou Prodolichotis pour les trois sous-familles des caviidés dans Pérez \& Pol [2012]) ou au contraire, augmenter et/ou créer des extensions fantômes (e.g. Prolagostomus pour les deux sous-familles des chinchillidés dans Kerber et al. [2018]);

- des taxons communs aux analyses mais qui y occupent des positions différentes. Par exemple dans Kerber et al. (2018), Garridomys et Eoviscaccia sont plus étroitement apparentés aux néoépiblemidés+chinchillidés et Scleromys osbornianus aux dinomyidés, tandis que ces taxons sont des chinchilloïdes basaux dans l'AG1. C'est également le cas majoritaire entre l'AG1 et le résultat de Verzi et al. (2016), où les taxons consi- 
TABLEAU 4. - Comparaison des âges de divergence impliqués par la topologie de l'AG1 avec ceux d'études précédentes: Arnal \& Vucetich (2015a), Kerber et al. (2018) et ce travail se basent uniquement sur des données morphologiques; Upham \& Patterson (2015), Sallam et al. (2009) et Huchon et al. (2007) uniquement sur des données moléculaires; Pérez \& Pol (2012) et Verzi et al. (2016) sur une combinaison des deux types de données. Les âges en gras sont contraints par des données fossiles. Remarque: certains âges proposés par Kerber et al. (2018: 6, fig. 4) apparaissent imprécis (i.e., surestimés) et ont été ajustés (valeurs entre crochets). Les âges sont donnés en million d'années (Ma).

\begin{tabular}{|c|c|c|c|c|c|c|c|c|}
\hline Clades & $\begin{array}{l}\text { Cette étude } \\
\text { (AG1) }\end{array}$ & $\begin{array}{l}\text { Kerber } \\
\text { et al. } \\
2017\end{array}$ & $\begin{array}{l}\text { Arnal \& } \\
\text { Vucetich } \\
2015 a\end{array}$ & $\begin{array}{l}\text { Verzi } \\
\text { et al. } \\
2016 \\
\end{array}$ & $\begin{array}{l}\text { Pérez } \\
\text { \& Pol } \\
2012\end{array}$ & $\begin{array}{l}\text { Upham \& } \\
\text { Patterson } \\
2015\end{array}$ & $\begin{array}{l}\text { Sallam et al. } \\
2009\end{array}$ & $\begin{array}{l}\text { Huchon et al. } \\
2007\end{array}$ \\
\hline Hystricognathi & c. 44 (Vacien) & - & - & - & - & $44,9(42,5-47,4)$ & $39,0(36,1-41,9)$ & $45,4(39,7-50,5)$ \\
\hline Caviomorpha & c. 43 (Vacien) & - & - & - & - & $42,0(41,1-43,3)$ & $36,1(33,4-39,0)$ & $42,7(37,3-47,2)$ \\
\hline $\begin{array}{c}\text { groupe couronne } \\
\text { Caviomorpha }\end{array}$ & c. 42 (Vacien) & - & - & 41 & - & - & - & - \\
\hline $\begin{array}{l}\text { Erethicavioi Boivin, } \\
\text { n. taxon }\end{array}$ & c. 37 (Mustersien) & - & - & 41 & - & - & - & - \\
\hline $\begin{array}{l}\text { Erethizontoidea- } \\
\text { Erethizontidae }\end{array}$ & $\begin{array}{l}\text { c. } 31 \text { (fin } \\
\text { Tinguiriricien) }\end{array}$ & - & - & 31,5 & - & $32,4(31,4-33,8)$ & $28,6(25,9-31,4)$ & $31,7(27,4-35,1)$ \\
\hline Erethizontinae & c. 30 & - & - & - & - & $17,5(11,3-24,0)$ & - & - \\
\hline Chaetomyinae & c. 30 & - & - & - & - & $17,5(11,3-24,0)$ & - & - \\
\hline Cavioidea & $\begin{array}{l}\text { c. } 31 \text { (fin } \\
\text { Tinguiriricien) }\end{array}$ & - & - & 31,5 & c. 31 & $32,4(31,4-33,8)$ & $28,6(25,9-31,4)$ & $31,7(27,4-35,1)$ \\
\hline $\begin{array}{l}\text { Dasyproctidae + } \\
\text { Cuniculidae }\end{array}$ & c. 30 & - & - & - & - & $25,2(24,3-26,7)$ & $23,1(20,6-26,0)$ & $24,1(20,1-28,0)$ \\
\hline Caviidae & c. 30 & - & - & - & c. 29 & $25,2(24,3-26,7)$ & $23,1(20,6-26,0)$ & $24,1(20,1-28,0)$ \\
\hline Caviinae & c. 18 & - & - & - & c. 15 & $17,6(14,9-20,6)$ & $14,6(12,4-17,2)$ & - \\
\hline $\begin{array}{c}\text { Hydrochoerinae + } \\
\text { Dolichotinae }\end{array}$ & c. 18 & - & - & - & c. 15 & $17,6(14,9-20,6)$ & $14,6(12,4-17,2)$ & - \\
\hline $\begin{array}{l}\text { Octochinchilloi } \\
\text { Boivin, n. taxon }\end{array}$ & c. 37 (Mustersien) & - & - & 41 & - & - & - & - \\
\hline Chinchilloidea & c. 36 (Mustersien) & $\begin{array}{l}\text { c. } 40 \\
{[33,5]}\end{array}$ & - & 31,5 & - & $32,8(31,4-34,5)$ & $29,0(26,2-31,7)$ & $33,3(28,9-36,3)$ \\
\hline $\begin{array}{c}\text { groupe couronne } \\
\text { Chinchilloidea }\end{array}$ & c. 14 & - & - & - & - & - & - & - \\
\hline Dinomyidae & c. 14 & c. 36 [33] & - & - & - & $19,7(14,8-24,7)$ & $19,9(16,5-23,2)$ & $21,4(17,9-25,6)$ \\
\hline Neoepiblemidae & c. 9 (Chasicoien) & c. 25 & - & - & - & - & - & - \\
\hline Chinchillinae & c. 9 (Chasicoien) & c. 24 & - & - & - & $12,3(9,3-15,9)$ & - & - \\
\hline Lagostominae & c. 9 (Chasicoien) & c. 24 & - & - & - & $12,3(9,3-15,9)$ & - & - \\
\hline Octodontoidea & c. 36 (Mustersien) & - & c. 37 & 31,5 & - & $32,8(31,4-34,5)$ & $29,0(26,2-31,7)$ & $33,3(28,9-36,3)$ \\
\hline Acaremyidae & c. 30 & - & - & - & - & - & - & - \\
\hline $\begin{array}{l}\text { Adelphomyidae, } \\
\text { n. stat. }\end{array}$ & c. 22 & - & - & - & - & - & - & - \\
\hline $\begin{array}{r}\text { groupe couronne } \\
\text { Octodontoidea }\end{array}$ & c. 22 & - & c. 35,5 & c. 31 & - & - & - & - \\
\hline Abrocomidae & c. 22 & - & - & - & - & $25,6(23,1-27,9)$ & - & - \\
\hline Ctenomyidae & $\begin{array}{l}\text { c. 9,5 } \\
\text { (Chasicoien) }\end{array}$ & - & - & c. 28 & - & $18,9(15,7-22,1)$ & - & - \\
\hline Octodontidae & $\begin{array}{l}\text { c. } 9,5 \\
\text { (Chasicoien) }\end{array}$ & - & - & c. 28 & - & $18,9(15,7-22,1)$ & - & - \\
\hline Echimyidae & c. 21,5 & - & - & c. 31 & - & $23,6(21,4-25,8)$ & $18,1(15,7-20,6)$ & $18,6(15,3-21,8)$ \\
\hline Capromyinae & c. 21 & - & - & - & - & 17,4 & - & - \\
\hline Euryzygomatomyinae & c. 21 & - & - & - & - & $16,7(15,1-18,1)$ & - & - \\
\hline Echimyinae & c. 21 & - & - & c. 31 & - & $18,2(17,1-19,3)$ & $12,3(9,9-14,7)$ & $12,4(9,7-15,3)$ \\
\hline Echimyini & $\begin{array}{l}\text { c. } 19 \\
\text { (Colhuehuapien) }\end{array}$ & - & - & c. 30 & - & $17,1(16,4-18,1)$ & - & - \\
\hline Myocastorini & c. 19 & - & - & c. 30 & - & $17,1(16,4-18,1)$ & - & - \\
\hline
\end{tabular}

dérés comme des octodontoïdes basaux dans l'AG1 sont situés à la base des différentes familles et sous-familles actuelles; - la présence de taxons dans l'AG1 provenant des gisements paléogènes de Contamana et de Tarapoto, qui n'avaient pas été intégrés aux analyses précédentes, notamment pour l'excellente raison qu'ils n'ont été décrits qu'entre-temps (Boivin et al. 2017a, b, 2018a). Au même titre que celle d'un groupe de branchement élargi, leur inclusion modifie profondément: i) la topologie des nouds basaux au sein des caviomorphes et de chacune des super-familles qui y sont reconnues; et ii) la polarité des changements morpho-anatomiques considérés.

Les âges de divergence observés dans l'AG1 sont majoritairement similaires à ceux d'études exclusivement fondées sur des données moléculaires et qui ont permis d'établir le squelette de la présente analyse (e.g. Huchon et al. 2007; Sallam et al. 2009; Upham \& Patterson 2015; Tableau 4). Dans ces analyses, ils correspondent 
souvent à des nœuds contraints par des données fossiles, mais pas uniquement (e.g. Sallam et al. 2009; Tableau 4). Néanmoins, certains âges d'apparition obtenus ici sont largement supérieurs (divergence Erethizontinae-Chaetomyinae) ou inférieurs (divergence Ctenomyidae-Octodontidae) en comparaison avec ces analyses (Tableau 4). Ces différences observées pouraient s'expliquer par le fait qu'ils correspondent à des nœuds non-contraints dans les études fondées sur des données moléculaires. En outre, une incomplétude du registre fossile ne peut être exclue dans le cas de la divergence Ctenomyidae-Octodontidae. Même s'il est généralement considéré que les cténomyidés et les octodontidés apparaissent au Miocène supérieur, en raison de l'enregistrement des premiers genres typiques de ces familles (Chasicomys, Xenodontomys et Pseudoplateomys; e.g. Simpson 1945; Vucetich et al. 1999; Arnal \& Pérez 2013), une origine plus ancienne des cténomyidés et des octodontidés pourrait être envisagée.

\section{Radiations et contexte paléoenvironnemental}

Les résultats de l'AG1 soutiennent une apparition rapide du groupe couronne des caviomorphes autour de $42 \mathrm{Ma}$, faisant suite à l'apparition des Caviomorpha (autour de $43 \mathrm{Ma}$ ) et à la divergence de plusieurs taxons basaux incluant notamment Canaanimys et Cachiyacuy. Le statut basal de ces deux caviomorphes, déjà évoqué et soutenu phylogénétiquement, au moins pour Canaanimys (Antoine et al. 2012), nécessitait d'être confirmé par de plus amples analyses (voir Vucetich et al. 2015a). Pour la première fois les AG1 et AG7 ont révélé l'existence de nombreux caviomorphes basaux: Pozomys, Eobranisamys, Eoespina, Tarapotomys, Shapajamys et Plesiosteiromys, tous situés aux basses latitudes (voir sections "Les groupes basaux des caviomorphes» et «Paléobiogéographie des caviomorphes au paléogène» ci-après). La plupart de ces taxons (Eobranisamys, Eoespina, Shapajamys et Plesiosteiromys) avaient préalablement été attribués à différentes super-familles sur la base de ressemblances dentaires avec certains de leurs représentants (Frailey \& Campbell 2004; Boivin et al. 2017b, 2018a). D'après les résultats des AG1 et AG7, ces ressemblances correspondraient soit à des symplésiomorphies (e.g. patron pentalophodonte des molaires supérieures d'Eobranisamys) ou à des convergences (e.g. patron tétralophodonte des molaires supérieures d'Eoespina) (voir section "Les groupes basaux des caviomorphes"). D'après les résultats des AG1 et AG7, ces taxons basaux se diversifieraient rapidement et persisteraient jusqu'au début de l'Oligocène inférieur (sur un intervalle d'environ c. $9 \mathrm{Ma}$ ), avec les genres Eoespina, Tarapotomys, Shapajamys et Eobranisamys, voire jusqu'à l'Oligocène supérieur avec Plesiosteiromys.

Les chinchilloïdes et les octodontoïdes sont présents dès la fin de l'Éocène. Une diversification basale très rapide transparaît pour les chinchilloïdes, correspondant à l'émergence des taxons Chambiramys et Eoincamys ou de formes apparentées (il est à noter qu'Eoincamys était jusqu'à présent attribué aux cavioïdes dasyproctidés; voir section "Les chinchillö̈des basaux»). Les octodontoïdes se seraient diversifiés plus tardivement, à l'Oligocène inférieur, conduisant notamment à la différenciation des taxons octodontoïdes déséadiens (Deseadomys, Platypittamys et Sallamys). À l'Oligocène inférieur, voire dès l'Éocène supérieur (voir section "Âges de divergence des principaux clades»), les éréthizontoïdes et cavioïdes seraient apparus, suivis de la mise en place des certaines familles (caviidés et éréthizontidés) et sous-familles (éréthizontinés et chaetomyinés) actuelles. De la toute fin de l'Oligocène supérieur jusqu'au Miocène inférieur, les octodontoïdes et très probablement les chinchilloïdes (voir section «Âges de divergence des principaux clades») aurient connu une grande phase de diversification, s'accompagnant de la différenciation de leurs groupes couronne respectifs. D'après l'AG1, l'origine du groupe couronne des caviidés serait d'âge Miocène inférieur. Comme pour les octodontoïdes et les chinchilloïdes, cette période serait également marquée par une diversification de certains cavioïdes basaux (non inclus dans l'AG1; e.g. Phanomys ou Eocardia), légèrement plus récente toutefois (Santacrucien, fin Miocène inférieur) selon les résultats de Pérez \& Pol (2012). Les éréthizontoïdes, et notamment les éréthizontinés, semblent également se diversifier à cette époque. Enfin, les octodontidés et les cténomyidés seraient au plus tard apparus au Miocène supérieur (voir section «Âges de divergence des principaux clades») et se diversifieraient à cette époque.

Trois périodes de radiation peuvent être reconnues (Fig. 9): - la première, à la fin de l'Éocène moyen, qui correspond à l'origine du groupe des caviomorphes, à la différenciation des premiers taxons basaux et à la mise en place du groupe couronne;

- la deuxième, de l'Éocène supérieur à l'Oligocène inférieur qui correspond à l'émergence des quatre super-familles actuelles; - la troisième, de la fin de l'Oligocène supérieur au Miocène inférieur, qui correspond à des diversifications plus récentes au sein des chinchilloïdes, des octodontoïdes et des éréthizontinés basaux, et à l'émergence du groupe couronne des caviidés, des octodontoïdes et très probablement des chinchilloïdes (voir section "Âges de divergence des principaux clades»).

Ces trois phases de diversification ne sont pas en adéquation avec celles reconnues par des auteurs précédents (Pérez \& Pol 2012; Arnal \& Vucetich 2015a; Verzi et al. 2016), et ce pour des raisons évoquées en section Âges de divergence des principaux clades. Bien que les différentes diversifications observées dans l'AG1 dépendent de l'échantillonnange taxonomique choisi ainsi que de la complétude du registre fossile, les présents résultats ont permis la mise en évidence de deux phases de diversification jusqu'ici non évoquées: la diversification basale des caviomorphes à l'Éocène moyen, et la diversification basale des chinchilloïdes autour de la transition Éocène-Oligocène.

D'autre part, ces radiations sont remarquablement concomitantes avec des évènements climatiques enregistrés à l'échelle du globe: l'optimum climatique de la fin de l'Éocène moyen (Middle Eocene Climatic Optimum, MECO), le refroidissement de la transition Éocène-Oligocène (abrégé ici EOTc) et le refroidissement de la transition Oligocène-Miocène (abrégé ici OMBc; Zachos et al. 2001 ; Bohaty \& Zachos 2003 ; Cramer et al. 2009; Fig. 9). Au contraire du MECO, les deux derniers évènements correspondent à des variations positives du $\delta 18 \mathrm{O}$ des carbonates marins, qui ont été, respectivement, nommées Oi-1G et Mi-1G par Miller et al. (1991). 


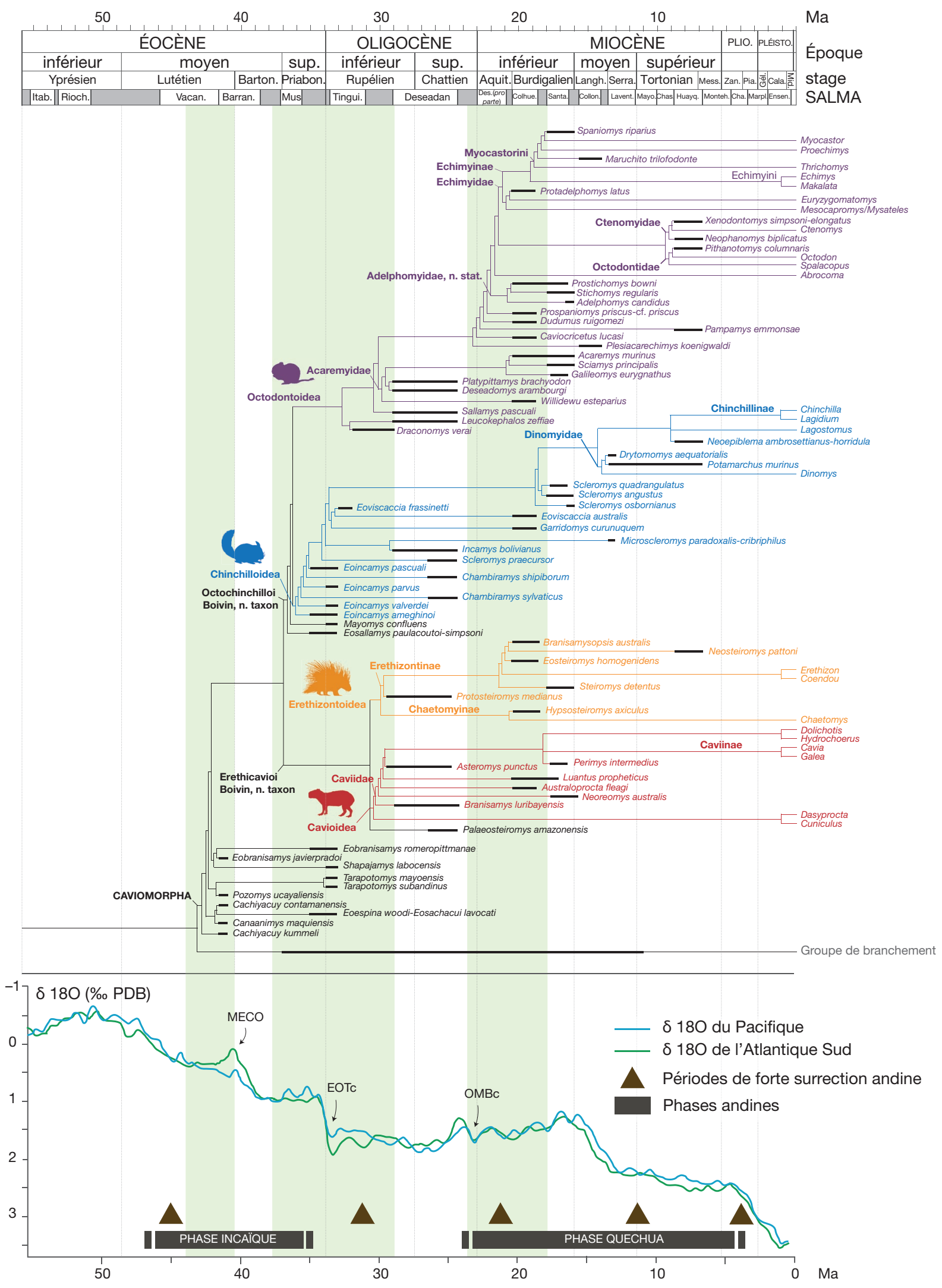

FIG. 9. - Mise en contexte géologique (Amérique du Sud; Hoorn et al. 2010a) et climatique (variations globales du $8180 ;$ Zachos et al. 2001, 2008) des trois phases de diversifications des caviomorphes (en vert) au Paléogène et Miocène. La topologie de l'arbre est celle obtenue dans l'AG1 (Fig. 5). Le code couleur est le même que celui de la Figure 3. Abréviations: MECO, optimum climatique de l'Éocène moyen; EOTc, refroidissement de la transition Éocène-Oligocène; $\mathrm{OMBc}$, refroidissement de la transition Oligocène-Miocène. 
Le MECO est un optimum climatique bref (c. $500 \mathrm{Ka})$ autour de 40-42 Ma, qui représente une réversion abrupte des températures par rapport à la tendance générale vers un refroidissement, faisant suite à l'optimum climatique de l'Éocène inférieur (Early Eocene Climatic Optimum, EECO; Bohaty \& Zachos 2003; Bohaty et al. 2009). Cet optimum correspondrait à un réchauffement de $c$. 4 à $6^{\circ}$ des eaux de surface et de profondeur moyenne (Bohaty et al. 2009). Il serait induit par une augmentation rapide du taux global de $\mathrm{CO}_{2}$ atmosphérique, pouvant être liée à des phénomènes tectoniques (e.g. Bohaty \& Zachos 2003; Bohaty et al. 2009). Une concordance de l'arrivée de ces rongeurs sur le continent sud-américain et de leur radiation initiale avec le Oi- $1 \mathrm{G}$ avait été proposée par Flynn \& Wyss (1998) ou Flynn et al. (2002). Cependant, la découverte récente de taxons de caviomorphes dans l'Éocène moyen terminal de Contamana en Amazonie péruvienne, et leur inclusion dans des analyses phylogénétiques (Antoine et al. 2012; Barbière \& Marivaux 2015; ce travail) révèlent que les premières phases de l'histoire évolutive des caviomorphes ont eu lieu beaucoup plus tôt, et à une période bien plus chaude et humide à l'échelle globale. Ces résultats sont en adéquation avec les biotopes des localités éocènes à rongeurs de Contamana, inférés à l'aide de données biologiques (e.g. palynomorphes, vertébrés associés) et abiotiques (e.g. faciès sédimentaire; Antoine et al. 2012, 2016). D'après l'AG1, l'arrivée des rongeurs hystricognathes en Amérique du Sud précèderait le MECO, et correspondrait à une période de stabilité thermique Atlantique-Pacifique. Cependant la différenciation originelle des premiers taxons basaux et la mise en place du groupe couronne coïncideraient bien avec le MECO.

L'EOTc correspond à un refroidissement rapide (c. $400 \mathrm{ka})$ et de grande ampleur $\left(4^{\circ} \mathrm{C}\right.$ environ; Zachos et al. 2001, 2008), même si l'existence d'un fort gradient est à soupçonner entre les régions de basses, moyennes et hautes latitudes. L'une des causes possibles généralement évoquée au EOTc est l'ouverture du passage de Drake entre l'Antarctique et l'Amérique du Sud, vers $34 \mathrm{Ma}$ (e.g. Livermore et al. 2005). Cette ouverture aurait permis la mise en place du courant circumpolaire antarctique, induisant un refroidissement global du climat, ainsi que la formation d'une calotte polaire pérenne sur l'Antarctique (Kennett 1977; Baker 2001; Sijp \& England 2004). Cette forte diminution des températures aurait été la principale cause des renouvellements taxonomiques observés à l'échelle mondiale dans les écosytèmes marins et terrestres de l'époque (la «Grande Coupure» de Stehlin 1909; Prothero \& Berggren 1992; Meng \& McKenna 1998). En Amérique du Sud, plusieurs changements floristiques et fauniques ont été mis en corrélation avec l'EOTc, particulièrement dans la partie sud de cette masse continentale (e.g. Olivero et al. 1998; Flynn et al. 2003; Barreda \& Palazzesi 2010; Goin et al. 2010) mais pas uniquement (Jaramillo et al. 2006, 2010). Aux hautes latitudes, alors que les paléoflores de l'Éocène supérieur sont encore marquées par la dominance de certains mégathermes, celles du Tinguiriricien (faunes de Tinguiririca et La Cantera) sont caractérisées par la prépondérence du microtherme Nothofagus et des gymnospermes (Olivero et al. 1998; Barreda \& Palazz- esi 2010). Les faunes de marsupiaux ont connu un important renouvellement à cette époque précise (Goin et al. 2010). À l'image de la "Grande Coupure» décrite en Europe (Stehlin 1909) et du «Mongolian Remodeling» en Asie Centrale (Meng \& McKenna 1998), Goin et al. (2010) ont proposé de nommer ces changements taxonomiques et écologiques majeurs, observés chez les faunes mammaliennes terrestres de Patagonie: «Bisagra Patagónica» («la charnière patagonienne»). Dans les régions tropicales de basses latitudes, une forte diminution de la diversité des plantes est enregistrée à la fin de l'Éocène et au début de l'Oligocène (Jaramillo et al. 2006, 2010). D'après l'AG1, la mise en place du groupe couronne des caviomorphes, des chinchilloïdes et des octodontoïdes seraient antérieures à l'EOTc, tandis que celle des cavioïdes et des éréthizontoïdes et la diversification basale des super-familles sont potentiellement concomitantes à cet évènement (voir sections « Résultats phylogénétiques et données stratigraphiques» et "Âges de divergence des principaux clades»). Ainsi, l'EOTc pourrait avoir constitué un facteur favorable à la diversification du groupe couronne des caviomorphes, observée à cette époque. Toutefois, cette hypothèse nécessiterait d'être confirmée par un registre fossile éocène plus étoffé (et des contextes environnementaux local et régional mieux contraints). Comme chez les ongulés natifs sud-américains, le développement de l'hypsodontie chez plusieurs caviomorphes de l'Oligocène (e.g. Cephalomys, Eoviscaccia, Litodontomys ou Scotamys) est généralement considéré comme une innovation évolutive clef du groupe, en lien avec l'ouverture et l'aridification des milieux suite à l'EOTc (e.g. Huchon \& Douzery 2001; Flynn et al. 2003; Antonelli et al. 2010) ou à une importante activité volcanique en Patagonie (Kay et al. 1999; Strömberg et al. 2013). Cependant, les données polliniques révèlent que l'expansion des milieux ouverts se serait produite beaucoup plus tardivement en Patagonie, au Miocène inférieur $\left(c .45^{\circ} \mathrm{S}\right.$; Barreda \& Palazzesi 2010; Palazzesi \& Barreda 2012; voir ci-dessous). Les plus anciens caviomorphes à couronnes hautes sont Eoviscaccia frassinettii et, dans une moindre mesure, les taxons amazoniens Eoincamys, Tarapotomys et Chinchilloidea indet., découverts dans les localités de Shapaja (Boivin et al. 2018a). Tous ces taxons sont subcontemporains et d'âge Oligocène inférieur. Comme précédemment signalé (Vucetich et al. 2010b), la première super-famille à développer l'hypsodontie serait celle des chinchilloïdes. Néanmoins, d'après l'AG1, une augmentation de la hauteur des couronnes pourrait intervenir en même temps chez les représentants amazoniens du groupe et chez certains caviomorphes basaux, comme Tarapotomys.

Le $\mathrm{OMBc}$ enregistré à la limite Oligocène-Miocène (c. 23 $\mathrm{Ma})$ représente un refroidissement très court $(c .200 \mathrm{ka})$ mais abrupt, sans doute lié à des facteurs orbitaux (i.e., paramètres de Milanković; Miller et al. 1991; Zachos et al. 2001). Il fait directement suite à l'optimum climatique de l'Oligocène supérieur et est suivi par une série de refroidissements intermittents de plus faible magnitude. D'après l'AG1, cet événement coïnciderait avec la diversification des quatre super-familles et l'apparition de nombreux taxons (documentés en Argentine et/ou au Chili). Des changements sont observés au niveau des communautés végétales du Miocène inférieur des hautes latitudes par rapport 


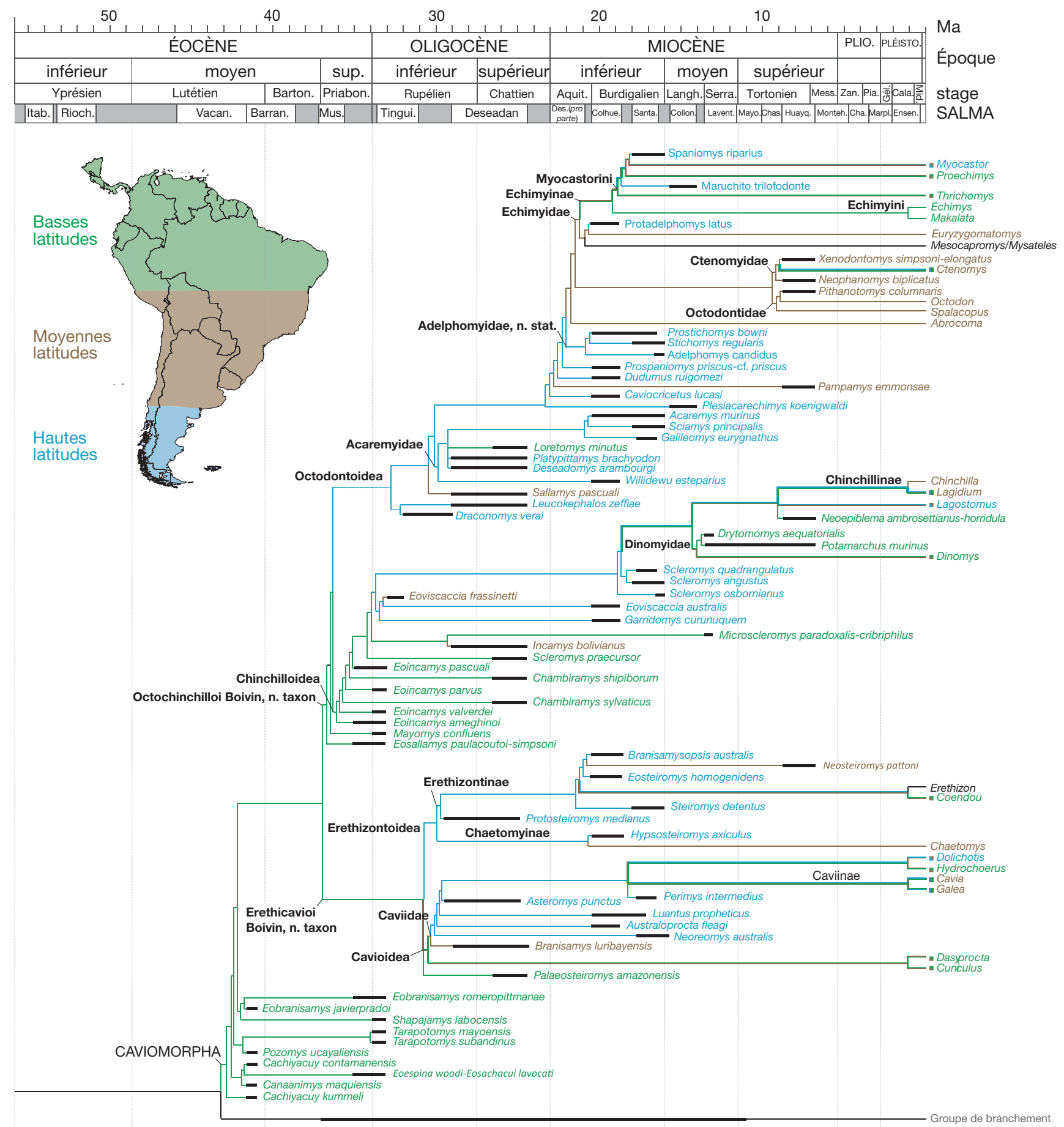

FIG. 10. - Mise en contexte paléogéographique de la topologie obtenue en AG9 (AG1 + Loretomys; voir Annexe 4).

à celles de la fin de l'Oligocène supérieur, et sont probablement directement en lien avec les évènements climatiques de ces périodes. En effet, les assemblages du Miocène inférieur sont caractérisés par l'expansion des plantes herbacées et arbustives, et le développement de végétaux adaptés à des conditions plus arides, suggérant un climat moins humide et une ouverture importante des milieux (Barreda \& Palazzesi 2010). Plusieurs caviomorphes du Miocène inférieur, découverts dans le sud du continent sud-américain, montrent des adaptations potentielles à de tels milieux. Par exemple, les octodontoïdes Protadelphomys et Willidewu ont une morphologie crânienne comparable à celle de formes modernes semi-fouisseuses vivant dans des milieux de savanes (Carterodon, Clyomys et Euryzygomatomys; Vucetich \& Verzi 1991). De plus, la tendance au développement d'une couronne dentaire haute dans les quatre superfamilles (e.g. Acaremys, Protadelphomys Sciamys, Hypsosteiromys, Scleromys, Cephalomyopsis et les caviidés euhypsodontes) aurait pu faciliter la colonisation par ces groupes de ces nouvelles 
prairies ouvertes (Candela \& Vucetich 2002; Vucetich et al. 2015a). Cependant, la forte activité volcanique des Andes à cette époque a également pu favoriser l'augmentation de la hauteur des couronnes dentaires de ces taxons en réponse à l'ajout de poussières abrasives à leur alimentation, lié au dépôt de cendres volcaniques sur la végétation (Vizcaíno et al. 2012; Candela \& Vucetich 2002; Vucetich et al. 2015a). Lapparition concomitante de milieux plus arides dans cette région aurait permis le développement de nouvelles niches, créant ainsi des opportunités de diversification.

Un autre facteur qui peut être mis en relation avec ces trois périodes de radiation des caviomorphes est l'orogenèse andine, et les perturbations environnementales associées (Fig. 9). Bien que les phases de surrection andine les plus spectaculaires soient bien plus tardives (Miocène supérieur-Pliocène inférieur; fin de la phase quechua), des phases plus anciennes de surrection marquées par un raccourcissement tectonique important sont également enregistrées (Jaillard \& Soler 1996). Certaines de ces phases sont concomitantes avec les première et troisième périodes de diversification des caviomorphes: la phase incaïque de l'Éocène moyen à l'Éocène supérieur et le début de la phase quechua pendant tout le Miocène inférieur (Steinmann 1929; Jaillard \& Soler 1996). Ces deux phases tectoniques concernent l'ensemble de la Cordillère, de la Colombie à la Patagonie (e.g., Cobbold \& Rossello 2003; Mora et al. 2010).

La phase incaïque serait la principale période d'orogenèse andine du Pérou (Noble et al. 1974, 1979; Mégard 1984). Or, il est intéressant de noter que les caviomorphes basaux reconnus dans l'AG1 et AG7, et qui se diversifieraient pendant cette phase, sont exclusivement constitués de taxons d'Amazonie péruvienne. La deuxième période de radiation empièterait sur la fin de la phase incaïque et une phase oligocène globale de quiescence andine. Cette phase de quiescence est marquée par la décroissance du raccourcissement de la Cordillère Ouest des Andes Centrales et potentiellement de celle des Andes du Sud (Cobbold \& Rossello 2003; voir Martinod et al. 2010). Cependant, le début d'un raccourcissement important de la Cordillère Est des Andes Centrales est observé à cette période (e.g. Lamb \& Hoke 1997; Roperch et al. 2006; Oncken et al. 2006). La formation de la Cordillère des Andes, qui est l'une des chaînes de montagnes les plus grandes et élevées du monde, a probablement eu un impact considérable sur les écosytèmes cénozoïques d'Amérique du Sud par le biais de variations climatiques, pluviométriques, chimiques [cycle du carbone notamment] et hydrographiques; Hoorn et al. 2010a). Elle aurait eu un rôle majeur dans l'origine et la diversification de la biodiversité sud-américaine et notamment amazonienne (e.g. Gentry 1982; Antonelli et al. 2009; Hoorn et al. 2010a, 2013).

La surrection d'une telle chaîne de montagne pourrait favoriser la diversification soit: i) directement par l'augmentation de l'hétérogénéité physiographique, en facilitant les dispersions d'espèces pré-adaptées, en créant de nombreuses opportunités de spéciations allopatriques des taxons des basses et des hautes altitudes, et par dispersion secondaire et radiation dans d'autres biomes des espèces nouvellement apparues (diversity pump); soit ii) indirectement en affectant les taux d'érosion, l'hydrologie et le climat à des échelles variées (locale, régionale et continentale; voir Antonelli et al. 2010; Hoorn et al. 2010a, 2013 et références associées). Ces modifications des taux d'érosion, hydrologiques et climatiques (e.g. Hoorn et al. 2010b; Roddaz et al. 2010; Vonhof \& Kaandorp 2010; Wesselingh et al. 2010) pourraient à leur tour avoir entraîné une hétérogénéité plus marquée des habitats et la constitution de barrières géographiques ayant à leur tour favorisé un isolement reproductif. Le rôle éventuel de plusieurs de ces mécanismes dans la mise en place des lignées modernes d'échimyidés au Miocène supérieur a été récemment discuté (Fabre et al. 2017). Des évènements de diversification observés chez d'autres groupes paraissent également avoir pu être concomitants avec des épisodes de surrection andine (e.g. Elias et al. 2009; McGuire et al. 2014).

En conclusion, les changements climatiques globaux et la surrection andine sont des facteurs susceptibles d'avoir favorisé les diversifications anciennes chez les rongeurs caviomorphes. De précédents auteurs avaient déjà évoqué leur rôle potentiel dans les variations de diversité cénozoïques observées chez ces rongeurs, ces deux facteurs pouvant agir de manière indépendante ou conjointe (e.g. Vucetich \& Verzi 1999; Vucetich et al. 1999; Pérez 2010b; Verzi et al. 2016; Vucetich et al. 2015a; Fabre et al. 2017). L'histoire évolutive du groupe a très probalement été complexe et influencée par une combinaison de facteurs biologiques et abiotiques, agissant à plus ou moins longs termes et à différentes échelles.

\section{PALÉOBIOGÉOGRAPHIE DES CAVIOMORPHES AU PALÉOGÈNE} Les caviomorphes basaux reconnus dans l'AG1 et AG7 proviennent tous d'Amazonie péruvienne (Fig. 10). Ces résultats soutiennent une diversification initiale du groupe restreinte aux basses latitudes du continent sud-américain. À partir du registre fossile disponible, ce constat pourrait être élargi aux chinchilloïdes, au contraire des octodontoïdes et des éréthizontoïdes, dont les premiers représentants connus sont au contraire connus sous de hautes latitudes (Fig. 10). L'origine des cavioïdes est plus ambiguë (i.e., basses ou moyennes latitudes selon l'AG1; Fig. 10). Comme évoqué à plusieurs reprises, Eopululo et Eopicure, découverts à Santa Rosa au Pérou et qui seraient, respectivement, les plus anciens représentants présumés des éréthizontoïdes et des cavioïdes, n’ont pas été inclus dans les analyses. Ainsi, une origine des éréthizontoïdes et des caviö̈des aux basses latitudes n'est pas exclue si ces taxons sont pris en considération (et leur appartenance aux super-familles correspondantes soutenue). Du fait de la position basale des taxons amazoniens Mayomys, Eosallamys et Palaeosteiromys au clade (Chinchilloidea, Octodontoidea) ou proche de la dichotomie Cavioidea-Erethizontoidea, une origine du groupe couronne des caviomorphes aux basses latitudes apparaît plus parcimonieuse (Fig. 10), même si la rareté du registre éocène des caviomorphes ne permet pas de conclure de manière certaine.

Les basses latitudes du continent sud-américain constitueraient le berceau des caviomorphes à partir duquel ils se seraient ensuite dispersés vers les moyennes et hautes latitudes. Le tempo et les acteurs de cette (ou ces) dispersion(s) restent difficiles à apprécier, mais nos résultats seraient plutôt en faveur de l'hypothèse d'expansions de leur aire géographique pour chacune des super-familles, indépendamment, et ce autour 
de la transition Éocène-Oligocène. En raison (du moins en partie) d'un registre fossile bien plus étoffé que dans les autres régions d'Amérique du Sud, les hautes latitudes apparaissent clairement comme étant un autre centre de diversification pour les quatre super-familles. Comme proposé par Arnal \& Vucetich (2015a), ces évènements de diversification pourraient être directement liés à cette (ou ces) dispersion(s), et à la colonisation subséquente de nouvelles niches écologiques par ces rongeurs. Les origines différentes des super-familles soutenues par l'AG1, notamment entre les éréthizontoïdes et les cavioïdes, et entre les chinchilloïdes et les octodontoïdes, laissent supposer un registre éocène-oligocène incomplet du groupe, concernant probablement à la fois les basses et les hautes latitudes. Le rôle mineur joué par les régions des moyennes latitudes en dépit de leur position centrale suggère également un enregistrement fossile lacunaire dans cette zone géographique. Plusieurs dispersions tardives entre ces trois régions sont mises en évidence à l'Oligocène avec les genres Sallamys (Salla, Bolivie; fin Oligocène inférieur-Oligocène supérieur) et Loretomys (CTA-32, Pérou; Oligocène supérieur), et au Miocène avec par exemple Neosteiromys (Chiquimil, Argentine; Miocène supérieur). En outre, plusieurs genres déséadiens sont communs entre les hautes et les moyennes latitudes (Asteromys/Cephalomys [voir section "Cephalomyidae: cavioïdes ou chinchilloïdes?»], Cephalomyopsis, Eoviscaccia, Incamys et Protosteiromys; Vucetich 1989; Bond et al. 1998; Bertrand et al. 2012; Vucetich et al. 2014, 2015a, b; Pérez et al. 2018), ainsi qu'entre les hautes et les basses latitudes (Deseadomys; Wood \& Patterson 1959; Boivin et al. 2017b) (Fig. 10). Ainsi, plusieurs dispersions auraient eu lieu à l'Oligocène entre ces régions et concerneraient différentes super-familles (au moins les octodontoïdes, les éréthizontoïdes et les chinchilloïdes), avec une nette expansion des aires de répartition correspondantes.

Il faut également noter la grande similitude entre les assemblages déséadiens de hautes (Cabeza Blanca, La Flecha, Laguna de los Machos et Pico Truncado) et moyennes latitudes (Arroyo Àvalos, Arroyo Maria Grande, Cerro Pan de Azúcar, Lacayani, Quebrada Fiera et Salla), par opposition aux faunes des basses latitudes (CTA-32, CTA-61 et Lircay; Pérez et al. 2018). Traditionnellement, ces similitudes/différences fauniques étaient interprétées comme représentant des différences écologiques et/ou temporelles entre les localités (Hoffstetter 1976; Patterson \& Wood 1982; Billet et al. 2008; Reguero \& Cerdeño 2005; Pujos \& De Iuliis 2007; Vucetich et al. 2015b). Les progrès en matière de datations des gisements déséadiens (MacFadden et al. 1985; Marshall \& Sempéré 1993; Flynn \& Swisher 1995; Kay et al. 1998; Shockey et al. 2009; Vucetich et al. 2014; Antoine et al. 2016; Pérez et al. 2018) ont permis de mettre en évidence que des différences temporelles existaient entre les localités déséadiennes, mais que ces dernières étaient assez réduites, notamment entre CTA-32, CTA-61, Cerro Pan de Azúcar, Salla et Scarritt Pocket (Pérez et al. 2018). Une combinaison de plusieurs facteurs historiques, paléobiogéographiques et environmentaux pourrait donc être à l'origine de ces similitudes et différences observées entre faunes déséadiennes de rongeurs caviomorphes (Pérez et al. 2018).

\section{SYSTÉMATIQUE}

Ordre RODENTIA Bowdich, 1821

Infraordre HYSTRICOGNATHI Tullberg, 1899

Parordre CAVIOMORPHA Wood, 1955

\section{Clade ERETHICAVIOI Boivin, n. taxon}

ÉTYMOLOGIE. - Coalescence entre "Erethizontoidea" et "Cavioidea", les deux super-familles actuelles qui sont rapportées au clade.

TAXONS INCLUS. - Clade regroupant les Erethizontoidea, les Cavioidea et taxons fossiles qui leur sont directement apparentés.

DiAGNOSE CLADISTIQUE. - Caviomorpha caractérisé par une courte excroissance du protocône sur la $\mathrm{P} 4$; un métacône non fusionné au postérolophe sur la P4; un métalophe présent sur la P4; une longueur de la M2 similaire à sa largeur ou plus longue; l'absence du bras postérieur du paracône sur les molaires supérieures; une longueur de la $\mathrm{p} 4$ supérieure à celle de la $\mathrm{m} 1$; les $\mathrm{dp} 4$ possédant un trigonide large; une entoconide et un hypoconide alignés sur les dp4; un long néomésolophide sur les molaires inférieures; et un entoconide aligné ou postérieur à l'hypocône sur les $\mathrm{m} 2$; une longeur courte du foramen incisif par rapport à celle des rangées dentaires.

Distribution. - De l'Éocène supérieur-Oligocène inférieur à nos jours, originaires d'Amérique du Sud et distribution naturelle étendue aux Amériques.

\section{Clade OCTOCHINCHILLOI Boivin, n. taxon}

ÉTYMOLOGIE. — Coalescence entre "Chinchilloidea» et «Octodontoidea", les deux super-familles actuelles qui sont rapportées au clade.

TAXONS InCLUS. - Clade regroupant les Chinchilloidea, les Octodontoidea et taxons fossiles qui leur sont directement apparentés.

Diagnose CladistiQue. — Caviomorpha caractérisé par l'absence de métacône sur les P4; et un métacône, lorsqu'il est présent, positionné plus lingualement au paracône sur les M2.

DisTRIBUTION. - De l'Éocène supérieur-Oligocène inférieur à nos jours, originaires d'Amérique du Sud et distribution naturelle étendue aux Amériques.

\section{Super-famille Octodontoidea Waterhouse, 1839}

Famille †ADELPHOMYIDAE Patterson \& Pascual, 1968, n. stat.

GENRE TYPE. - Adelphomys candidus Ameghino, 1887.

ÉTYMOLOGIE. - En référence à son genre type.

AUTRES TAXONS INCLUS. - Stichomys regularis Ameghino, 1887; Prostichomys bowni Kramarz, 2001.

Diagnose Cladistique. - Octodontoidea caractérisé par une extension moyenne de l'hypoflexus sur des M1-2 modérément usées; une connexion du bras postérieur du protoconide avec le néomésolophide sur les dp4; un alignement de l'hypolophide avec l'ectolophide sur les molaires inférieures; et une crête massétérique modérément développée sur le dentaire.

Distribution. - Sarmiento Formation, Pinturas Formation et Santa Cruz Formation, Argentine; Miocène inférieur. 
Super-famille CHINCHILlOIDEA Bennett, 1833

Genre Maquiamys Boivin, n. gen.

urn:Isid:zoobank.org:act:FB5181F5-2057-4C80-8C82-57C57D01C196

EsPÈCE TYPE. - Maquiamys praecursor (Boivin, 2017) n. comb. (Boivin et al. 2017b).

AUTRES ESPÈCES DU GENRE. - Uniquement l'espèce type.

ÉTYMOLOGIE. - En référence à l'anticlinal de Maquía sur le flanc sud-ouest duquel affleure CTA-61, la localité type du taxon.

Diagnose GÉNÉRIQUe. - Identique à celle de l'espèce type.

Maquiamys praecursor (Boivin, 2017) n. comb.

Scleromys praecursor Boivin in Boivin, Marivaux, Candela, Orliac, Pujos, Salas-Gismondi, Tejada-Lara \& Antoine, 2017b: 91.

Chinchilloidea indet. - Antoine et al. 2016: données supplémentaires, 11.

?Chinchilloidea - Antoine et al. 2016: données supplémentaires, 11.

Caviomorpha indet. - Antoine et al. 2016: données supplémentaires, 11 .

Chinchilloidea indet. - Antoine et al. 2017: données supplémentaires, 9 .

?Chinchilloidea - Antoine et al. 2017: données supplémentaires, 9.

Caviomorpha indet. - Antoine et al. 2017: données supplémentaires, 9.

Holotype. - MUSM 2888, M1 ou M2 droite; Boivin et al. 2017b: fig. 5Q.

MATÉRIEL EXAMINÉ. - MUSM 2887 (fragment de molaire supérieure gauche; Boivin et al. 2017b: fig. 5U); MUSM 2895 (M1 ou M2 gauche; Boivin et al. 2017b: fig. 5V); MUSM 2889 (M1 ou M2 droite; Boivin et al. 2017b: fig. 5R).

LOCALITÉ TYPE ET ÂGE. —CTA-61, Oligocène supérieur, située dans la région de Contamana, département du Loreto, Pérou.

Diagnose CLADistiQue. - Chinchilloïde de taille moyenne, caractérisé par des molaires supérieures avec une couronne protohypsodonte, des lophes très épais et fortement obliques et une épaisseur homogène de l'émail des bords de l'hypoflexus. Le protolophe lingual et le métacône sont absents sur les molaires supérieures. La longueur des M1 et M2 est supérieure ou égale à leur largeur.

\section{CLASSIFICATION DES CAVIOMORPHES FOSSILES}

La classification proposée ci-dessous n'est pas exhaustive et elle est fondée sur les résultats cladistiques des différentes analyses (AG0-AG9) obtenus dans ce présent article.

\section{Parvordre CAVIOMORPHA Wood, 1955}

Cachiyacuy kummeli Antoine, Marivaux, Croft, Billet, Ganerød, Jaramillo, Martin, Orliac, Tejada-Lara, Altamirano, Duranthon, Fanjat, Rousse \& Salas-Gismondi, 2012;
Cachiyacuy contamanensis Antoine, Marivaux, Croft, Billet, Ganerød, Jaramillo, Martin, Orliac, Tejada-Lara, Altamirano, Duranthon, Fanjat, Rousse \& Salas-Gismondi, 2012;

Canaanimys maquiensis Antoine, Marivaux, Croft, Billet, Ganerød, Jaramillo, Martin, Orliac, Tejada-Lara, Altamirano, Duranthon, Fanjat, Rousse \& Salas-Gismondi, 2012;

Eoespina woodi Frailey \& Campbell, 2004

Eosachacui lavocati Frailey \& Campbell, 2004

Pozomys ucayaliensis Boivin, 2017

Tarapotomys subandinus Boivin, Marivaux, Pujos, Salas-Gismondi, Tejada-Lara, Varas-Malca \& Antoine, 2018

Tarapotomys mayoensis Boivin, Marivaux, Pujos, Salas-Gismondi, Tejada-Lara, Varas-Malca \& Antoine, 2018

Eobranisamys javierpradoi Boivin, 2017

Eobranisamys romeropittmanae Frailey \& Campbell, 2004

Shapajamys labocensis Boivin, Marivaux, Pujos, Salas-Gismondi, Tejada-Lara, Varas-Malca \& Antoine, 2018

Plesiosteiromys newelli Boivin, 2017

Asteromys punctus Wood \& Patterson, 1959

Famille CepHalomyidae Ameghino, 1897 Litodontomys chubutomys Wood \& Patterson, 1959

Cephalomys arcidens Wood \& Patterson, 1959

«Asteromys» bolivianus (Lavocat, 1976)

? Soriamys gaimanensis Kramarz, 2001

Clade ERETHICAVIOI Boivin, n. taxon

Palaeosteiromys amazonensis Boivin, 2017

Super-famille ERETHIZONTOIDEA Bonaparte, 1845

Famille ERETHIZONTIDEA Bonaparte, 1845

Sous-famille ERETHizONTINEA Thomas, 1897

Protosteiromys medianus Wood \& Patterson, 1959

Steiromys detentus Ameghino, 1887

Eosteiromys homogenidens Ameghino, 1902

Branisamyopsis australis Candela, 2003

Branisamyopsis praesigmoides Candela, 2003

Neosteiromys pattoni Candela, 2004

Sous-famille CHAETOMyinae Thomas, 1897

Hypsosteiromys axiculus Ameghino, 1902

Super-famille CAVIOIDEA Fischer de Waldheim, 1817

Famille CAVIIDAE Waterhouse, 1839

Branisamys luribayensis Hoffstetter \& Lavocat, 1970

Neoreomys australis Ameghino, 1887

Australoprocta fleaglei Kramarz, 1998

Luantus propheticus Kramarz, 2006

Clade OCTOCHINCHILLOI Boivin, n. taxon

Eosallamys paulacoutoi Frailey \& Campbell, 2004

Eosallamys simpsoni Frailey \& Campbell, 2004

Mayomys confluens Boivin, Marivaux, Pujos, Salas-Gismondi, Tejada-Lara, Varas-Malca \& Antoine, 2018

Super-famille CHINCHILlOIDEA Bennett, 1833

Eoincamys ameghinoi Frailey \& Campbell, 2004 
Eoincamys valverdei Boivin, Marivaux, Pujos, Salas-Gismondi, Tejada-Lara, Varas-Malca \& Antoine, 2018

Eoincamys parvus Boivin, Marivaux, Pujos, Salas-Gismondi, Tejada-Lara, Varas-Malca \& Antoine, 2018

Eoincamys pascuali Frailey \& Campbell, 2004

Chambiramys sylvaticus Boivin, 2017

Chambiramys shipiborum Boivin, 2017

Maquiamys praecursor (Boivin, 2017) n. comb.

Incamys bolivianus Hoffstetter \& Lavocat, 1970

Microscleromys paradoxalis Walton, 1997

Microscleromys cribriphilus Walton, 1997

Garridomys curunuquem Kramarz, Vucetich \& Arnal, 2013

Eoviscaccia frassinettii Bertrand, Flynn, Croft \& Wyss, 2012

Eoviscaccia australis Vucetich, 1989

Scleromys quadrangulatus Kramarz, 2006

Scleromys angustus Ameghino, 1887

Scleromys osbornianus Ameghino, 1894

Neoepiblema ambrosettianus (Ameghino, 1889)

Neoepiblema horridula (Ameghino, 1886)

Famille DinOMYIDAE Alston, 1876

Drytomomys aequatorialis Anthony, 1922

Potamarchus murinus Burmeister, 1885

Super-famille OctOdONTOIDEA Waterhouse, 1839

Leucokephalos zeffiae Vucetich, Dozo, Arnal \& Pérez, 2015

Draconomys verai Vucetich, Vieytes, Pérez \& Carlini, 2010

Sallamys pascuali Hoffstetter \& Lavocat, 1970

Plesiacarechimys koenigswaldi Vucetich \& Vieytes, 2006

Caviocricetus lucasi Vucetich \& Verzi, 1996

Dudumus ruigomezi Arnal, Kramarz, Vucetich \& Vieytes, 2014

Prospaniomys priscus Ameghino, 1902

Pampamys emmonsae Verzi, Vucetich \& Montalvo, 1995

Famille †ACAREMYIDAE Wood, 1949

Acaremys murinus Ameghino, 1887

Sciamys principalis Ameghino, 1887

Galileomys eurygnathus Kramarz, 2004

Platypittamys brachyodon Wood, 1949

Deseadomys arambourgi Wood \& Patterson, 1959

Willidewu esteparius Vucetich \& Verzi, 1991

Loretomys minutus Boivin, 2017

Famille †Adelphomyidae Patterson \& Pascual, 1968, n. stat.

Adelphomys candidus Ameghino, 1887

Stichomys regularis Ameghino, 1887

Prostichomys bowni Kramarz, 2001

Famille OCTOdONTIDAE Waterhouse, 1839

Pithanotomys columnaris Ameghino, 1887

Famille CTENOMYIDAE Lesson, 1842

Xenodontomys simpsoni Kraglievich, 1961

Xenodontomys elongatus Verzi, Montalvo \& Tiranti, 2003

Neophanomys biplicatus Rovereto, 1914
Famille ECHIMYIDAE Gray, 1825

Protadelphomys latus Ameghino, 1902

Sous-famille ECHIMYINAE Gray, 1825

Tribu Myocastorini Fabre, Upham, Emmons, Justy, Leite, Loss, Orlando, Tilak, Patterson \& Douzery, 2017

Maruchito trilofodonte Vucetich, Mazzoni \& Pardiñas, 1993

Spaniomys riparius Ameghino, 1887

\section{CONCLUSIONS}

Dans le cadre de ce travail, une analyse cladistique de grande ampleur, incluant un grand nombre de familles représentant les quatre super-familles, a été réalisée. Les résultats montrent que les taxons paléogènes de Contamana et de Tarapoto/Shapaja en Amazonie péruvienne appartiennent à des taxons basaux du clade Caviomorpha ou des super-familles. Les taxons enregistrés dans les localités de la fin de l'Éocène moyen de Contamana sont probablement tous des représentants basaux des caviomorphes. D'autres formes basales seraient également rencontrées à Santa Rosa (Eobranisamys romeropittmanae, Eoespina woodi-Eosachacui lavocati) et Tarapoto/Shapaja (Tarapotomys). L'attribution suprafamiliale de certains taxons amazoniens récemment décrits (Plesiosteiromys, Mayomys et potentiellement Palaeosteiromys) demeure ambiguë car leur position phylogénétique respective est en partiel désaccord avec les conclusions systématiques initiales - établies sans évaluation cladistique des caractères dentaires - et concernant leur rang suprafamilial, telles que proposées par Boivin et al. (2017b, 2018a). De plus, les résultats phylogénétiques obtenus ici permettent une première révision des cavioïdes dasyproctidés. L'ensemble des taxons fossiles originellement décrits comme étant des membres de cette famille et inclus dans cette analyse, se placent au sein des groupes basaux des caviomorphes (Eobranisamys romeropittmanae et Eobranisamys javierpradoi), des chinchilloïdes (Incamys bolivianus et Microscleromys) ou des cavioïdes non dasyproctidés (Branisamys luribayensis et Australoprocta fleaglei).

Les régions de basses latitudes du continent sud-américain paraissent être le centre d'origine des caviomorphes et le lieu d'une première diversification basale du groupe à la fin de l'Éocène moyen. Les quatre super-familles émergeraient ensuite à l'Éocène supérieur-Oligocène inférieur, témoignant ainsi d'une deuxième radiation du groupe. C'est à cette période que les caviomorphes se sont de toute évidence dispersés aux moyennes et hautes latitudes. L'origine géographique des super-familles est ambiguë, excepté pour les chinchilloïdes qui auraient émergé et se seraient initialement différenciés dans les régions de basses latitudes. Les régions de hautes latitudes constitueraient ainsi un deuxième centre de diversification. Une troisième radiation aurait eu lieu autour de 
la transition Oligocène-Miocène et correspondrait à des diversifications plus récentes des groupes basaux des chinchilloïdes, des octodontoïdes, des éréthizontinés et à l'émergence du groupe couronne des caviidés, de celui des octodontoïdes, et très probablement de celui des chinchilloïdes. Il est intéressant de souligner ici que ces trois événements majeurs de diversification apparaissent concomitants avec des évènements climatiques globaux et des périodes intenses de surrection andine. Enfin, plusieurs dispersions tardives entre les régions de basses, moyennes et hautes latitudes ont été également mises en évidence à l'Oligocène et au Miocène, se traduisant le plus souvent par une expansion des aires de distribution des clades concernés plutôt que par leur translation.

Ce travail a également permis d'éclairer plusieurs points de l'histoire évolutive des rongeurs caviomorphes. Cependant, des parts d'incertitude demeurent notamment vis-à-vis des âges d'apparition du groupe couronne des caviomorphes et des quatre super-familles, ainsi que de l'origine géographique de ces groupes. Les résultats phylogénétiques obtenus, replacés dans leur contexte stratigraphique indiquent que le registre éocène et oligocène inférieur est clairement incomplet pour le groupe, et ce à toutes les latitudes (et altitudes). Des efforts sur le terrain devront donc être poursuivis pour parfaire le registre fossile de ce groupe, notamment durant l'intervalle clef Éocène moyen terminal-Oligocène inférieur, afin d'apporter de nouvelles données critiques, essentielles à la compréhension des débuts de l'histoire évolutive des caviomorphes.

Enfin, grâce à ce corpus élargi de données, il sera possible d'envisager d'autres méthodes de reconstruction phylogénétique telles que les méthodes probabilistes (méthode du maximum de vraisemblance et approche bayésienne), par le biais desquelles des caractères moléculaires pourraient être ajoutés à la matrice. L'alliance de taxons modernes et fossiles permettrait l'utilisation d'une approche tip dating (ou total evidence dating) afin de mieux estimer les âges de divergence des nœuds. En outre, des méta-analyses probabilistes de diversité (e.g. birth-death models) pourront aussi permettre de tester statistiquement si une corrélation existe entre les périodes de diversification connues par le groupes et des facteurs abiotiques potentiels tels que les évènements climatiques globaux et les périodes intenses de surrection andine. Ces modèles couplés à des analyses biogéographiques permettront de tester l'impact de ces facteurs et d'autres facteurs potentiels (e.g. facteurs biologiques) sur ces radiations et les évènements de dispersion et vicariance passées chez les caviomorphes.

\section{Remerciements}

Nous remercions tous les membres de l'équipe internationnale qui ont contribué à la découverte et à l'exploitation des localités d'Amazonie péruvienne depuis plus d'une décennie, ainsi que toutes les personnes qui nous ont aidés sur le terrain. Merci à Suzanne Jiquel, Bernard Marandat (ISE-M), Christine Argot, Guillaume Billet, Violaine Nicolas, Aurélie Verguin (MNHN), César Chacaltana, Luz Marina Tejada-Medina (INGEMMET, Pérou), Alejandro Kramarz (MACN, Argentine), Marcelo Reguero, Itatí Olivares (MLP, Argentine) et Bernardino Mamani Quispe (MNHN-Bol, Bolivie) de nous avoir permis d'accéder aux collections dont ils ont la charge. Nous adressons également nos sincères remerciements à François Pujos (IANIGLA, Argentine) pour son aide et son investissement à long-terme dans la collaboration avec le MNHN-Bol ; à Rubén Andrade Flores (MNHN$\mathrm{Bol}$ ) et Céline Robinet (MLP) pour leur aide précieuse pendant notre séjour au MLP et au MNHN-Bol; et à Lenna Defend (ISE-M) pour l'inventaire et le conditionnement de la collection UM des rongeurs de Salla. Un grand merci à François Catzeflis (ISE-M) pour le prêt de son matériel, et à Lionel Hautier, Pierre-Henri Fabre (ISE-M), Adriana Candela et C. Robinet (MLP) pour les photos de plusieurs spécimens de collections. Nous sommes reconnaissants envers Anne-Lise Charruault, S. Jiquel et Sandra Unal (ISE-M), ainsi qu'envers Jacques Michaux (ISE-M), Pauline Coster (UK, USA), Hesham Sallam (Mansura University, Egypte) et Guiomar Vucetich (MLP) d'avoir enrichi notre matériel de comparaison grâce à la confection et/ou l'accès à de nombreux moulages. Un grand merci à Maeva Leitwein (IBIS, Québec), Clémentine François (LEHNA, France), P.-H. Fabre et la Plate-forme MBB/ISE-M Calcul \& Bioinformatique et le SR2I pour leur aide concernant l'utilisation du cluster. Merci à F. Catzeflis, P.-H. Fabre, L. Hautier, Maeva Orliac et Helder Gomes Rodrigues (ISE-M), G. Billet (MNHN), Michelle Arnal, Cecilia Deschamps, Luciano Rasia, et María G. Vucetich (MLP), María Encarnación Pérez (MEF, Argentine) et F. Pujos pour nos discussions passionantes à propos des hystricognathes et des rongeurs en général, et de la phylogénie. Enfin, nous remercions très chaleureusement Véronique Barriel (MNHN), A. Candela, A. Kramarz, E. J.P. Douzery (ISE-M), Erik Seiffert (USC, USA) pour leur relecture appréciée d'une version étendue de ce travail, et leurs remarques et conseils avisés dans le cadre de la thèse de doctorat de MB. Nous remercions également le Rédacteur en chef, Didier Merle (MNHN), et le secrétaire d'édition, Emmanuel Côtez (MNHN), de la revue Geodiversitas ainsi que V. Barriel et le second rapporteur anonyme pour leur relecture de ce long travail et leurs commentaires qui ont permis son amélioration. Ce travail a été financé par la Leakey Foundation, par la National Geographic Society, par l'École Doctorale SIBAGHE/ Gaïa de l'Université de Montpellier, par la subvention 'Investissements d'Avenir' gérée par l'Agence Nationale de la Recherche (CEBA, ANR-10-LABX-0025-01), par le projet SOURCE (CEBA, appel 2016) et par les programmes de collaboration internationale CoopIntEER CNRS/CONICET et ECOS-SUD/FONCyT. Publication ISEM 2018-249. 


\section{RÉFÉRENCES}

Alston E. R. 1876. - On the classification of the order Glires. Proceedings of the Zoological Society of London 1: 61-98. https:// doi.org/10.1111/j.1096-3642.1876.tb02543.x

Ameghino F. 1886. - Contribuciones al conocimiento de los mamíferos fósiles de los terrenos terciarios antiguos del Paraná. Boletín de la Academiade Ciencias de Córdoba 9: 1-228. http:// www.biodiversitylibrary.org/page/26274027

AMEghino F. 1887. - Enumeración sistematica de las especies de mamíferos fósiles coleccionados por Carlos Ameghino en los terrenos eocenos de Patagonia austral y depositados en el museo de La Plata. Boletín del Museo de La Plata 1: 1-26.

Ameghino F. 1889. - Contribución al conocimiento de los mamíferos fósiles de la República Argentina. Actas de la Academia Nacional de Ciencias de Córdoba 6: 1-1027. https://doi. org/10.5962/bhl.title. 121288

AMEGHINO F. 1894. — Énumération synoptique des espèces de mammiferes fossiles des formations éocènes de Patagonie. Boletín de la Academia Nacional de Ciencias en Córdoba 13: 259-455. https://doi.org/10.5962/bhl.title.77348

Ameghino F. 1897. — Mamíferos cretáceos de la Argentina. Segunda contribución al conocimiento de la fauna mastológica de las capas con restos de Pyrotherium. Boletín Instituto Geográfico Argentino 18: 406-429, 431-521.

AMEGHINO F. 1902. - Première contribution à la connaissance de la faune mammalogique des couches à Colpodon. Boletín de la Academia Nacional de Ciencias en Córdoba 17: 71-138. http:// www.biodiversitylibrary.org/page/26370391

Ameghino F. 1903. - Los diprotodontes del orden de los plagiaulacoideos y el origen de los roedores y de los polimastodontes. Anales del Museo Nacional de Historia Natural de Buenos Aires, série III, 2: 81-192. https://biodiversitylibrary.org/page/4497426

AnTHONy H. E. 1922. - A new fossil rodent from Ecuador. American Museum Novitates 35: 1-4. http://hdl.handle.net/2246/3250

ANTOINE P.-O. 2002. - Phylogénie et évolution des Elasmotheriina (Mammalia, Rhinocerotidae). Muséum national d'Histoire naturelle, Paris, 369 p. (Mémoires du Muséum national d'Histoire naturelle; 188).

ANTOINE P.-O. 2003. - Middle Miocene elasmotheriine Rhinocerotidae from China and Mongolia: taxonomic revision and phylogenetic relationships. Zoologica Scripta 32: 95-118. https:// doi.org/10.1046/j.1463-6409.2003.00106.x

Antoine P.-O., Marivaux L., Croft D. A., Billet G., Ganerød M., Jaramillo C., Martin T., Orliac M. J., Tejada-Lara J., Altamirano A. J., Duranthon F., Fanjat G., Rousse S. \& SALAS-GisMONDI R. 2012. — Middle Eocene rodents from Peruvian Amazonia reveal the pattern and timing of caviomorph origins and biogeography. Proceedings of the Royal Society of London B 279 (1732): 1319-1326. https://doi.org/10.1098/rspb.2011.1732

Antoine P.-O., Abello M., Adnet S., Altamirano Sierra A. J., Baby P., Billet G., Boivin M., Calderóne Y., Candela A., Chabain J., Corfu F., Croft D. A., Ganerød M., Jaramillo C., Klaus S., Marivaux L., Navarrete R. E., Orliac M. J., Parra F., Pérez M. E., Pujos F., Rage J.-C., Ravel A., Robinet C., Roddaz M., Tejada-Lara J. V., VÉlez-Juarbe J., Wesselingh F. P. \& SAlas-Gismondi R. 2016. — A 60-million-year Cenozoic history of western Amazonian ecosystems in Contamana, eastern Peru. Gondwana Research 31:30-59. https:// doi.org/10.1016/j.gr.2015.11.001

Antoine P.-O., Salas-Gismondi R., Pujos F., Ganerød M. \& MarivaUX L. 2017. - Western Amazonia as a hotspot of mammalian biodiversity throughout the Cenozoic. Journal of Mammalian Evolution 24 (1): 5-17. https://doi.org/10.1007/ s10914-016-9333-1

Antonelli A., Nylander J.A., PersSOn C. \& SANMARTín I. 2009. Tracing the impact of the Andean uplift on Neotropical plant evolution. Proceedings of the National Academy of Sciences USA 106 (24): 9749-9754. https://doi.org/10.1073/pnas.0811421106
Antonelli A., Quijada-Mascareñas A., Crawford A. J., Bates J. M., VelAZCO P. M. \& WÜSTER W. 2010. — Molecular studies and phylogeography of Amazonian tetrapods and their relation to geological and climatic models, in HOORN C. \& WeSSELINGH F. P. (eds), Amazonia, Landscape and Species Evolution: A Look into the Past. Blackwell-Wiley, Hoboken: 386-404.

ArNal M. \& Kramarz A. G. 2011. - First complete skull of an octodontoid (Rodentia, Caviomorpha) from the Early Miocene of South America and its bearing in the early evolution of Octodontoidea. Geobios 44: 435-444. https://doi.org/10.1016/j. geobios.2010.12.003

Arnal M. \& Pérez M. E. 2013. - A new acaremyid rodent (Hystricognathi: Octodontoidea) from the Middle Miocene of Patagonia (South America) and considerations on the early evolution of Octodontoidea. Zootaxa 3616 (2): 119-34. https:// doi.org/10.11646/zootaxa.3616.2.2

ARNAL M. \& VUCETICH M. G. 2011. — First record of supernumerary teeth in South American fossil rodents. Journal of Vertebrate Paleontology 31 (4): 925-927. https://doi.org/10.1080/02724634.2011.576732

ARnal M. \& Vucetich M. G. 2015a. - Main radiation events in Pan-Octodontoidea (Rodentia, Caviomorpha). Zoological Journal of the Linnean Society 175 (3): 587-606. https://doi. org/10.1111/zoj.12288

Arnal M. \& Vucetich M. G. 2015b. - Revision of the fossil rodent Acaremys Ameghino, 1887 (Hystricognathi, Octodontoidea, Acaremyidae) from the Miocene of Patagonia (Argentina) and the description of a new acaremyid. Historical Biology 27: 42-59. https://doi.org/10.1080/08912963.2013.863881

Arnal M., Kramarz A. G., Vucetich M. G. \& Vieytes E. C. 2014. - A new early Miocene octodontoid rodent (Hystricognathi, Caviomorpha) from Patagonia (Argentina) and a reassessment of the early evolution of Octodontoidea. Journal of Vertebrate Paleontology 34: 397-406. https://doi.org/10.1080/0 2724634.2013.808203

Arnal M., Vucetich M. G., Croft D. A., Bargo M. S., FerniCola J. C. \& VizcaínO S. F. 2017. - Systematic revision and evolutionary history of Acarechimys Patterson in Kraglievich, 1965 (Rodentia, Caviomorpha, Octodontoidea). Ameghiniana 54 (3): 307-330. https://doi.org/10.5710/AMGH.17.02.2017.3048

BAKER P. F. 2001. - Scotia Sea regional tectonic evolution: implications for mantle flow and palaeocirculation. Earth Science Reviews 55 (1): 1-39. https://doi.org/10.1016/S0012-8252(01)00055-1

BARBIĖRE F. \& MARIVAUX L. 2015. - Phylogeny and evolutionary history of hystricognathous rodents from the Old World during the Tertiary: new insights into the emergence of modern "phiomorph" families, in COX P. \& HAUTIER L. (eds), Evolution of the Rodents: Advances in Phylogeny, Functional Morphology and Development. Cambridge University Press, Cambridge: 87-138. https://doi.org/10.1017/CBO9781107360150.005

BARREDA V. \& PAlazzesi L. 2010. - Vegetation during the Eocene-Miocene interval in central Patagonia: a context of mammal evolution, in MAdDEN R. H., CARLini A. A., VuCETICH M. G. \& KAY R. F. (eds), The Paleontology of Gran Barranca, Evolution and Environmental Change through the Middle Cenozoic of Patagonia. Cambridge University Press, Cambridge: 375-382.

BenneTt E. T. 1833. - On the Chinchillidae, a family of herbivorous Rodentia, and on a new genus referrible to it. Transactions of the Zoological Society of London 1: 35-64. https://doi. org/10.1111/j.1096-3642.1835.tb00602.x

Bertrand O. C., Flynn J. J., Croft D. A. \& Wyss A. R. 2012. Two new taxa (Caviomorpha, Rodentia) from the Early Oligocene Tinguiririca fauna (Chile). American Museum Novitates 3750: 1-36. https://doi.org/10.1206/3750.2

Billet G., Muizon C. DE \& Mamani Quispe B. 2008. — Late Oligocene mesotheriids (Mammalia, Notoungulata) from Salla and Lacayani (Bolivia): implications for basal mesotheriid phylogeny and distribution. Zoological Journal of the Linnean Society 152: 153-200. https://doi.org/10.1111/j.1096-3642.2007.00388.x 
Bohaty S. M. \& Zachos J. C. 2003. - Significant Southern Ocean warming event in the late middle Eocene. Geology 31 (11): 1017-1020. https://doi.org/10.1130/G19800.1

Bohaty S. M., Zachos J. C., Florindo F. \& Delaney M. L. 2009. - Coupled greenhouse warming and deep-sea acidification in the middle Eocene. Paleoceanography 24 (2): 1-16. https://doi. org/10.1029/2008PA001676

Borvin M. 2017. - Rongeurs paléogènes d'Amazonie péruvienne: anatomie, systématique, phylogénie et paléobiogéographie. Volume de Thèse, Université de Montpellier, France.

BoIVIN M. \& MARIVAUX L. 2018. - Dental homologies and evolutionary transformations in Caviomorpha (Hystricognathi, Rodentia): new data from the Paleogene of Peruvian Amazonia. Historical Biology. https://doi.org/10.1080/08912963.2018.1506778

Boivin M., Marivaux L., Orliac M. J., Pujos F., Salas-GisMONDI R., TeJadA-LaRA J. V. \& ANTOINe P.-O. 2017a. — Late middle Eocene caviomorph rodents from Contamana, Peruvian Amazonia. Palaeontology Electronica 20.1.19A: 1-50. https://doi. org/10.26879/742

Boivin M., Marivaux L., Candela A. M., Orliac M. J., Pujos F., Salas-Gismondi R., Tejada-Lara J. V. \& Antoine P.-O. 2017b. - Late Oligocene caviomorph rodents from Contamana, Peruvian Amazonia. Papers in Palaeontology 3: 69-109. https:// doi.org/10.1002/spp2.1068

Boivin M., Marivaux L., Pujos F., Salas-Gismondi R., TejadaLara J. V., Varas-Malca R. M. \& Antoine P.-O. 2018a. Early Oligocene caviomorph rodents from Shapaja, Peruvian Amazonia. Palaeontographica Abteilung A 311 (1-6): 87-156. https://doi.org/10.1127/pala/2018/0075

Boivin M., Marivaux L., Salas-Gismondi R., Vieytes E. C. \& AnTOINE P.-O. 2018b. - Incisor enamel microstructure of Paleogene caviomorph rodents from Contamana and Shapaja (Peruvian Amazonia). Journal of Mammalian Evolution. https:// doi.org/10.1007/s10914-018-9430-4

BonaparTe C. L. J. L. 1845. - Cata logo methodico dei mammiferi Europei. Luigi di Giacomo Pirola, Milano, 36 p. https://doi. org/10.5962/bhl.title.77311

Bond M., LÓpez G., Reguero M. A., Sciliato-Yané G. J. \& VuceTich M. G. 1998. — Los mamíferos de la formación Fray Bentos (edad mamífero Deseadense, Oligoceno superior?) de las provincias de Corrientes y Entre Ríos, Argentina. Asociación Paleontológica Argentina, Publicación Especial 5: 41-50.

Bond M., TEJEDOR M. F., MACFAdDEN JR K. E., CHORNOGUbSKy L., Novo N. \& GoIN F. 2015. — Eocene primates of South America and the African origins of New World monkeys. Nature 520 (7548): 538-541. https://doi.org/10.1038/nature14120

BREMER K. 1994. - Branch support and tree stability. Cladistics 10 (3): 295-304. https://doi.org/10.1111/j.1096-0031.1994.tb00179.x

BRUIJN H. DE, ÜNAY E., SARAÇ G. \& YÏlmaZ A. 2003. — A rodent assemblage from the Eo/Oligocene boundary interval near Süngülü, Lesser Caucasus, Turkey, in LóPEZ-MARTínez N., PelÁEZ-CAmpomanes P. \& HenándeZ FERnÁndeZ M. (eds), Coloquios de Paleontologia en Honor al Dr Remmert Daams Surrounding Fossil Mammals: Dating, Evolution and Paleoenvirontment. Madrid: Facultad de Ciencias Geologicas Departamento de Paleontologia: 47-76.

BURMEISTER G. 1885. — Exámen crítico de los mamíferos y reptiles fósiles denominados por D. Augusto Bravard y mencionados en su obra precedente. Annales del Museo Nacional de Buenos Aires 3: 93-174. https://biodiversitylibrary.org/page/45614456

Busker F. \& DOZO M. T. 2017. — First confirmed record of Incamys bolivianus (Caviomorpha, Chinchilloidea) in the Deseadan of Patagonia (Argentina). Ameghiniana 54 (6): 706-712. https:// doi.org/10.5710/AMGH.24.05.2017.3098

Busker F. \& Dozo M. T. 2018. - Rediscovering a forgotten rodent of Patagonia and its phylogenetic implications. Journal of Systematic Palaeontology. https://doi.org/10.1080/14772019 .2018 .1457727
Busker F., Pérez M. E., Krause J. M. \& Vucetich M. G. 2017. First record of Banderomys leanzai Kramarz, 2005 (Rodentia, Caviomorpha) in Chubut Province, Patagonia (Argentina). Revista del Museo Argentino de Ciencias Naturales 19 (2): 121129. https://doi.org/10.22179/REVMACN.19.533

CANDEla A. M. 1999. - The evolution of the molar pattern of the Erethizontidae (Rodentia, Hystricognathi) and the validity of Parasteiromys Ameghino, 1904. Palaeovertebrata 28 (1): 53-73.

Candela A. M. 2000. - Los Erethizontidae (Rodentia, Hystricognathi) fósiles de Argentina. Sistemática e historia evolutiva y biogeográfica. Volume de Thèse non publié, Universidad Nacional de La Plata, Argentina.

CANDELA A. M. 2001. - First cladistic analysis of the extinct and living porcupines (Rodentia, Erethizontidae). Abstracts Sixth International Congress of Vertebrate Morphology (Jena, Germany). Journal of Morphology 248: 213. https://doi.org/10.1002/jmor.1031

CANDELA A. M. 2003. - A new porcupine (Rodentia, Erethizontidae) from the early-middle Miocene of Patagonia. Ameghiniana 40: 483-494.

Candela A. M. 2004. - A new giant Porcupine (Rodentia, Erethizontidae) from the Late Miocene of Argentina. Journal of Vertebrate Paleontology 24 (3): 732-741. https://doi.org/10.167 1/0272-4634(2004)024[0732:ANGPRE]2.0.CO;2

CANDELAA. M. 2016. - Analyzing the impact of conflictive dental characters on the phylogeny of octodontoid rodents. Acta Palaeontologica Polonica 61 (2): 455-468. https://doi.org/10.4202/app.00113.2014

CANDEla A. M. \& NASIF N. L. 2006. - Systematics and biogeographic significance of Drytomomys typicus (Scalabrini in Ameghino, 1889) nov. comb., a Miocene Dinomyidae (Rodentia, Hystricognathi) from Northeast of Argentina. Neues Jahrbuch für Geologie und Paläontologie Monatshefte 3: 165-181.

CANDElA A. M. \& Picasso M. B. 2008. - Functional anatomy of the limbs of Erethizontidae (Rodentia, Caviomorpha): indicators of locomotor behavior in Miocene porcupines. Journal of Morphology 269 (5): 552-593. https://doi.org/10.1002/jmor.10606

Candela A. M. \& Rasia L. L. 2012. - Tooth morphology of Echimyidae (Rodentia, Caviomorpha): homology assessments, fossils, and evolution. Zoological Journal of the Linnean Society 164: 451-480. https://doi.org/10.1111/j.1096-3642.2011.00762.x

CANDEla A. M. \& Vucetich M. G. 2002. - Hypsosteiromys (Rodentia, Hystricognathi) from the early Miocene of Patagonia (Argentina), the only Erethizontidae with a tendency to hypsodonty. Geobios 35 (1): 153-161. https://doi.org/10.1016/S0016-6995(02)00017-7

Candela A. M., Rasia L. L. \& Pérez M. E. 2012. — Early Miocene Paleobiology in Patagonia: Paleobiology of Santacrucian caviomorph rodents: a morphofunctional approach, in VIZCAínO S. F., KaY R. F. \& BARGO M. S. (eds), Early Miocene Paleobiology in Patagonia: High-Latitude Paleocommunities of the Santa Cruz Formation. Cambridge University Press, New York: 287-305. https://doi.org/10.1017/CBO9780511667381.016

Carvalho G. A. \& Salles L. O. 2004. - Relationships among extant and fossil echimyids (Rodentia: Hystricognathi). Zoological Journal of the Linnean Society 142 (4): 445-477. https://doi. org/10.1111/j.1096-3642.2004.00150.x

CERDEÑo E. 2011. - Quebrada Fiera (Mendoza), an important paleobiogeographic center in the South American late Oligocene. Estudios Geológicos 67 (2): 375-384. https://doi.org/10.3989/ egeol.40519.194

Cobbold P. R. \& Rossello E. A. 2003. - Aptian to recent compressional deformation, foothills of the Neuquen Basin, Argentina. Marine and Petroleum Geology 20: 429-443. https:// doi.org/10.1016/S0264-8172(03)00077-1

Coster P., Benammi M., Lazzari V., Billet G., Martin T., Salem M., Bilal A. A., Chaimanee Y., Schuster M., ValenTIN X., BRUNET M. \& JAEGER J.-J. 2010. — Gaudeamus lavocati sp. nov. (Rodentia, Hystricognathi) from the early Oligocene of Zallah, Libya: first African caviomorph? Naturwissenschaften 97 (8): 697-706. https://doi.org/10.1007/s00114-010-0683-x 
Coster P., Benammi M., Salem M., Bilal A. A., Chaimanee Y., Valentin X., Brunet M. \& Jaeger J.-J. 2012. - New hystricognathous rodents from the early Oligocene of central Libya (Zallah Oasis, Sahara Desert): systematic, phylogenetic, and biochronologic implications. Annals of Carnegie Museum 80 (3): 239-259.

Cramer B. S., Toggweiler J. R., Wright J. D., Katz M. E. \& Miller K. G. 2009. - Ocean overturning since the Late Cretaceous: inferences from a new benthic foraminiferal isotope compilation. Paleoceanography 24 (4): 1-14. https://doi. org/10.1029/2008PA001683

CrofT D. A., FlynN J. J. \& WYSS A. R. 2008. — The Tinguiririca fauna of Chile and the early stages of "modernization" of South American mammal faunas. Arquivos do Museu Nacional, Rio de Janeiro 66: 191-211. http://www.biodiversitylibrary.org/ page/57388661

Croft D. A., Chick J. M. \& Anaya F. 2011. — New Middle Miocene caviomorph rodents from Quebrada Honda, Bolivia. Journal of Mammalian Evolution 18: 245-268. https://doi.org/10.1007/ s10914-011-9164-z

DARLU P. \& TASSY P. 1993. - La reconstruction phylogénétique. Concepts et méthodes. Masson, Paris, 245 p.

Dawson M. D., LI C. K. \& QI T. 1984. — Eocene ctenodactyloid rodents (Mammalia) of Eastern and Central Asia, in MENGEL R. M. (ed.), Papers in Vertebrate Paleontology Honoring Robert Warren Wilson. Carnegie Museum of Natural History, Pittsburgh: 138150. https://biodiversitylibrary.org/page/52301996

Deschamps C. M. \& Tomassini R. L. 2016. — Late Cenozoic vertebrates from the southern Pampean Region: systematic and bio-chronostratigraphic update, in MARTínez M. \& Olivera D. (eds), Palinología del Meso-Cenozoico de Argentina - Volumen en homenaje a Mirta Elena Quattrocchio. Publicación Electrónica de la Asociación Paleontológica Argentina 16 (2): 202-225. http:// dx.doi.org/10.5710/PEAPA.06.05.2016.113

Dozo M. T. 1995. - El encéfalo de un Cephalomyidae (Rodentia, Caviomorpha) de la Edad Colhuehuapense: primer análisis paleoneurológico de un roedor caviomorfo y sus implicancias filogenéticas. Resúmenes XI Jornadas Argentinas de Paleontología de Vertebrados, Tucumán: 30.

Elias M., Joron M., Willmott K., Silva-Brandao K. L., Kaiser V., Arias C. F., Gomez Piñerez L. M., Uribe S., Brower A. V. Z., Freitas A. V. L. \& Jiggins C. D. 2009. - Out of the Andes: patterns of diversification in clearwing butterflies. Molecular Ecology 18 (8): 1716-1729. https://doi.org/10.1111/j.1365294X.2009.04149.x

EMmONS L. H. 2005. - A revision of the genera of arboreal Echimyidae (Rodentia: Echimyidae, Echimyinae), with descriptions of two new genera, in LACEY E. A. \& MYers P. (eds), Mammalian Diversification: From Chromosomes to Phylogeography (A Celebration of the Career of James L. Patton). Publications in Zoology 133: 247-310. http://hdl.handle. net/10088/12847

Fabre P.-H., Hautier L., Dimitrov D. \& Douzery E. J. 2012. A glimpse on the pattern of rodent diversification: a phylogenetic approach. BMC Evolutionary Biology 12 (1): 88. https://doi. org/10.1186/1471-2148-12-88

Fabre P.-H., Upham N. S., Emmons L. H., Justy F., Leite Y. L. R., loss A. C., Orlando L., Tilak M.-K., Patterson B. D. \& DOUZERY E. J. P. 2017. — Mitogenomic phylogeny, diversification, and biogeography of South American spiny rats. Molecular Biology and Evolution 34 (3): 613-633. https://doi.org/10.1093/ molbev/msw261

FIELDS R. W. 1957. - Hystricomorph rodents from the late Miocene of Colombia, South America. University of California Publications in Geological Sciences 32: 273-404.

Fischer De Waldheim G. 1817. — Adversaria Zoologica. Mémoires de la Société impériale des Naturalistes de Moscou 5: 357-428. https://biodiversitylibrary.org/page/10128982
Flynn J. \& Swisher C. 1995. - Cenozoic South American land-mammals ages: correlation to global geochronologies, in Bergreen W. A., Kent D. V. \& Handerbold J. (eds), Geochronoly, Time Scale, and Correlation: Framework for a Historical Geology. SEPM Special Publication 54: 317-333. https://doi. org/10.2110/pec.95.04.0317

FLYNN J. J. \& WYSS A. R. 1998. — Recent advances in South American mammalian paleontology. Trends in Ecology \& Evolution 13 (11): 449-454. https://doi.org/10.1016/S0169-5347(98)01457-8

FlYNN L. J., JACOBS L. L. \& CHEEMA I. U. 1986. — Baluchimyinae, a new ctenodactyloid rodent subfamily from the Miocene of Baluchistan. American Museum Novitates 2841: 1-58. http:// hdl.handle.net/2246/3576

Flynn J. J., NovaceK M. J., Dodson H. E., Frassinetti D. McKenna M. C., Norell M. A., Sears K. E., Swisher C. C. III \& WYSS A. R. 2002. - A new fossil mammal assemblage from the southern Chilean Andes: implications for geology, geochronology, and tectonics. Journal of South American Earth Sciences 15: 285-302. https://doi.org/10.1016/S0895-9811(02)00043-3

Flynn J. J., Wyss R., Croft D. A. \& Charrier R. 2003. - The Tinguiririca Fauna, Chile: biochronology, paleoecology, biogeography, and a new earliest Oligocene South American Land Mammal 'Age'. Palaeogeography, Palaeoclimatology, Palaeoecology 195: 229-259. https://doi.org/10.1016/S0031-0182(03)00360-2

FlynN J. J., Charrier R., Croft D. A., Gans P. B., Herriott T. M., Wertheim J. A. \& Wyss A. R. 2008. - Chronologic implications of new Miocene mammals from the Cura-Mallín and Trapa Trapa formations, Laguna del Laja area, south central Chile. Journal of South American Earth Sciences 26: 412-423. https://doi.org/10.1016/j.jsames.2008.05.006

Frailey C. D. \& CAMPBELL K. E. 2004. - Paleogene rodents from Amazonian Peru: the Santa Rosa local fauna, in CAMPBELl K. E. (ed.), The Paleogene Mammalian Fauna of Santa Rosa, Amazonian Peru. Natural History Museum of Los Angeles County, Science Series 40: 71-130.

GENTRY A. H. 1982. - Neotropical floristic diversity: phytogeographical connections between Central and South America, Pleistocene climatic fluctuations, or an accident of the Andean orogeny? Annals of the Missouri Botanical Garden 69 (3): $557-$ 593. https://doi.org/10.2307/2399084

Goin F. J. \& Candela A. M. 2004. - New Paleogene marsupials from the Amazon Basin of eastern Peru, in Campbell K.E. (ed.), The Paleogene Mammalian Fauna of Santa Rosa, Amazonian Peru. Natural History Museum of Los Angeles County, Science Series 40: 15-60.

Goin F. J., Abello M. A. \& Chornogubsky L. 2010. - Middle Tertiary marsupials from Central Patagonia (Early Oligocene of Gran Barranca): understanding South America's Grande Coupure, in Madden R. H., Carlini A. A., Vucetich M. G. \& KAY R. F. (eds), The Paleontology of Gran Barranca, Evolution and Environmental Change through the Middle Cenozoic of Patagonia. Cambridge University Press, Cambridge: 69-107.

GRAY J. E. 1825. - An outline of an attempt at the disposition of Mammalia into tribes and families, with a list of the genera apparently appertaining to each tribe. Annals of Philosophy, n.s. ser. 2, 10: 337-344. https://biodiversitylibrary.org/page/2531525

HARTENBERGER J.-L. 1975. - Nouvelles découvertes de rongeurs dans le Déseadien (Oligocène inférieur) de Salla Luribay (Bolivie). Comptes rendus hebdomadaires des Séances de l'Académie des Sciences, série D, 280: 427-430. https://gallica.bnf.fr/ark:/12148/ bpt6k5814218t/f463.item

Hill J. E. 1935. - The cranial foramina in rodents. Journal of Mammalogy 16 (2): 121-129. https://doi.org/10.2307/1374358

HOFFSTETTER R. 1976. - Rongeurs caviomorphes de l'Oligocène de Bolivie. Palaeovertebrata 7: 1-14.

Hoffstetter R. \& Lavocat R. 1970. - Découverte dans le Déséadien de Bolivie des genres pentalophodontes appuyant les affinités africaines des rongeurs caviomorphes. Comptes Rendus de l'Académie des Sciences, Paris 271: 172-175. https://gallica. bnf.fr/ark:/12148/bpt6k6398040d/f218.item 
Hoorn C., Wesselingh F. P., Ter Steege H., Bermudez M. A., Mora A., Sevink J., Sanmartín I., Sanchez-Meseguer A., Anderson C. L., Figueiredo J. P., Jaramillo C., Riff D., Negri F. R., Hooghiemstra H., Lundberg J., Stadler T., SÄRKINEN T. \& ANTONELli A. 2010a. - Amazonia through time: Andean uplift, climate change, landscape evolution, and biodiversity. Science 330 (6006): 927-931. https://doi.org/10.1126/ science. 1194585

Hoorn C., Wesselingh F. P., Hovikoski J. \& Guerrero J. 2010b. - The development of the Amazonian mega-wetland (Miocene; Brazil, Colombia, Peru, Bolivia), in HoOrN C. \& Wesselingh F. P. (eds), Amazonia, Landscape and Species Evolution: A Look into the Past. Blackwell-Wiley, Hoboken: 123-142.

Hoorn C., Mosbrugger V., Mulch A. \& Antonelli A. 2013. Biodiversity from mountain building. Nature Geoscience 6 (3): 154. https://doi.org/10.1038/ngeo1742

Horovitz I., SÁnchez-Villagra M., Martin T. \& Aguilera O. 2006. - The fossil record of Phoberomys pattersoni Mones 1980 (Mammalia, Rodentia) from Urumaco (Late Miocene, Venezuela), with an analysis of its phylogenetic relationships. Journal of Systematic Palaeontology 4 (3): 293-306. https://doi. org/10.1017/S1477201906001908

Houle A. 1998. - Floating islands: a mode of long-distance dispersal for small and medium-sized terrestrial vertebrates. Diversity and Distributions 4 (4/5): 201-216. https://www.jstor. $\mathrm{org} / \mathrm{stable} / 2999827$

Houle A. 1999. - The Origin of Platyrrhines: An Evaluation of the Antarctic Scenario and the Floating Island Model. American Journal of Physical Anthropology 109: 541-559. https:// doi.org/10.1002/(SICI)1096-8644(199908)109:4<541::AIDAJPA9>3.0.CO;2-N

Huchon D. \& Douzery E. J. 2001. - From the Old World to the New World: a molecular chronicle of the phylogeny and biogeography of hystricognath rodents. Molecular Phylogenetics and Evolution 20 (2): 238-251. https://doi.org/10.1006/ mpev.2001.0961

Huchon D., Chevret P., Jordan U., Kilpatrick C. W., RanWEZ V., JENKINS P. D., Brosius J. \& SChMitZ J. 2007. — Multiple molecular evidences for a living mammalian fossil. Proceedings of the National Academy of Sciences 104 (18): 7495-7499. https:// doi.org/10.1073/pnas.0701289104

Hussain S. T., Bruijn H. DE \& Leinders J. M. 1978. — Middle Eocene rodents from the Kala Chitta Range (Punjab, Pakistan). Palaeontology 81: 101-112. https://www.jstor.org/ stable/1304193

Jaeger J.-J., Marivaux L., Salem M., Bilal A. A., Chaimanee Y., Marandat B., Valentin X., Duringer P., SChuster M., Benammi M., Métais E. \& Brunet M. 2010. - New rodent assemblages from the Eocene Dur at-Talah escarpment (Sahara of Central Libya): systematic, biochronologic and paleobiogeographic implications. Zoological Journal of the Linnean Society 160: 195-213. https://doi.org/10.1111/j.10963642.2009.00600.x

Jaillard E. \& Soler P. 1996. — Cretaceous to early Paleogene tectonic evolution of the northern Central Andes (0-18 degrees S) and its relations to geodynamic. Tectonophysics 259: 41-53. https://doi.org/10.1016/0040-1951(95)00107-7

Jaramillo C., Rueda M. J. \& Mora G. 2006. - Cenozoic plant diversity in the Neotropics. Science 311 (5769): 1893-1896. https://doi.org/10.1126/science. 1121380

Jaramillo C., Hoorn C., Silva S. A., Leite F., Herrera F., Quiroz L., Dino R. \& ANTONioli L. 2010. - The origin of the modern Amazon rainforest: implications of the palynological and palaeobotanical record, in HoOrN C. \& WesselingH F. P. (eds), Amazonia, Landscape and Species Evolution: A Look into the Past. Blackwell-Wiley, Hoboken: 317-334.

KaY B. R. F. 2015. - New World monkey origins. Science 347 (6226): 1068-1069. https://doi.org/10.1126/science.aaa9217
Kay R. F., Macfadden B. J., Madden R. H., Sandeman H. \& ANAYA F. 1998. - Revised age of the Salla beds, Bolivia, and its bearing on the age of the Deseadan South American Land Mammal "Age". Journal of Vertebrate Paleontology 18 (1): 189-199. https://doi.org/10.1080/02724634.1998.10011043

Kay R. F., Madden R. H., Vucetich M. G., Carlini A. A., MaZzoni M. M., Re G. H., Heizler M. \& Sandeman H. 1999. Revised geochronology of the Casamayoran South American Land Mammal Age: climatic and biotic implications. Proceedings of the National Academy of Sciences 96 (23): 13235-13240. https://doi. org/10.1073/pnas.96.23.13235

KAY B. R. F., ViZCAÍnO S. F. \& BARGO M. S. 2012. - A review of the paleoenvironment and paleoecology of the Miocene Santa Cruz Formation, in VIZCAÍNO S. F., KAY R. F. \& BARGO M. S. (eds), Early Miocene Paleobiology in Patagonia. Cambridge, Cambridge University Press: 331-365. https://doi.org/10.1017/ CBO9780511667381.018

KENNETT J. P. 1977. - Cenozoic evolution of Antarctic glaciation, the circum-Antarctic Ocean, and their impact on global paleoceanography. Journal of Geophysical Research: Solid Earth 82 (27): 3843-3860. https://doi.org/10.1029/JC082i027p03843

Kerber L., Mayer E. L., Ribeiro A. M. \& Vucetich M. G. 2016. Late Quaternary caviomorph rodents (Rodentia: Hystricognathi) from the Serra da Capivara, northeastern Brazil, with description of a new taxon. Historical Biology 28 (4): 439-458. https://doi. org/10.1080/08912963.2014.967766

Kerber L., Negri F. R., Ribeiro A. M., Nasif N., SouZa-Filho J. P. \& FERIGOLO J. 2017. — Tropical fossil caviomorph rodents from the southwestern Brazilian Amazonia in the context of the South American faunas: systematics, biochronology, and paleobiogeography. Journal of Mammalian Evolution 24 (1): 57-70. https://doi.org/10.1007/s10914-016-9340-2

Kerber L., Bissaro Júnior M. C., Negri F. R., Souza-Filho J. P. De, Guilherme E. \& Hsiou A. S. 2018. - A new rodent (Caviomorpha: Dinomyidae) from the upper Miocene of southwestern Brazilian Amazonia. Historical Biology 30 (7): 985-993. https://doi.org/10.1080/08912963.2017.1327529

KRAGLIEVICH J. L. 1961. - Un nuevo roedor octodóntido del Eocuartario de Salinas Chicas (Provincia de Buenos Aires). Revista de la Asociación Geológica Argentina 15 (1-2): 15-41.

Kramarz A. G. 1998. - Un nuevo dasyproctidae (Rodentia, Caviomorpha) del Mioceno inferior de Patagonia. Ameghiniana 35 (2): 181-192.

Kramarz A. G. 2001a. - Revision of the family Cephalomyidae (Rodentia, Caviomorpha) and new cephalomyids from the Early Miocene of Patagonia. Palaeovertebrata 30: 51-88.

Kramarz A. G. 2001b. - Registro de Eoviscaccia (Rodentia, Chinchillidae) en estratos colhuehuapenses de Patagonia, Argentina. Ameghiniana 38 (3): 237-242.

Kramarz A. G. 2001c. - Un nuevo roedor Adelphomyinae (Hystricognathi, Echimyidae) del Mioceno Medio-Inferior de Patagonia, Argentina. Ameghiniana 38 (2): 163-168.

Kramarz A. G. 2002. — Roedores chinchilloideos (Hystricognathi) de la Formación Pinturas, Mioceno temprano-medio de la provincia de Santa Cruz, Argentina. Revista del Museo Argentino de Ciencias Naturales nueva serie 4 (2): 167-180. https://doi. org/10.22179/REVMACN.4.9

KramaRZ A. G. 2004. — Octodontoids and erethizontoids (Rodentia, Hystricognathi) from the Pinturas Formation, Early-Middle Miocene of Patagonia, Argentina. Ameghiniana 41: 199-216.

KramarZ A. G. 2005. - A primitive cephalomyid hystricognath rodent from the early Miocene of northern Patagonia, Argentina. Acta Palaeontologica Polonica 50 (2): 249-258.

Kramarz A. G. 2006a. — Eocardiids (Rodentia, Hystricognathi) from the Pinturas Formation, late early Miocene of Patagonia, Argentina. Journal of Vertebrate Paleontology 26 (3): 770-778. https://doi.org/10.1671/0272-4634(2006)26[770:ERHFT$\mathrm{P}] 2.0 . \mathrm{CO} ; 2$ 
Kramarz A. G. 2006b. - Neoreomys and Scleromys (Rodentia, Hystricognathi) from the Pinturas Formation, late Early Miocene of Patagonia, Argentina. Revista del Museo Argentino de Ciencias Naturales 8 (1): 53-62. https://doi.org/10.22179/ REVMACN.8.356

Kramarz A. G., Vucetich M. G., Carlini A. A., Ciancio M. R., Abello M. A., Deschamps C. M. \& Gelfo J. N. 2010. A new fauna at the top of the Gran Barranca sequence and its biochronological significance, in MADDEN R. H., CARLINI A. A., Vucetich M. G. \& KaY R. F (eds), The Paleontology of Gran Barranca, Evolution and Environmental Change through the Middle Cenozoic of Patagonia. Cambridge University Press, Cambridge: 264-277.

Kramarz A. G., Vucetich M. G. \& Arnal M. 2013. - A new early Miocene chinchilloid hystricognath rodent; an approach to the understanding of the early chinchillid dental evolution. Journal of Mammalian Evolution 20: 249-261. https://doi.org/10.1007/ s10914-012-9215-0

LAMB S. \& HoKE L. 1997. - Origin of the high plateau in the Central Andes, Bolivia, South America. Tectonics 16: 623-649. https://doi.org/10.1029/97TC00495

LANDRY S. O. 1957. - The interrelationship of the New and Old World hystricomorph rodents. University of California Publications in Geological Sciences 32 (5): 273-404.

LAVOCAT R. 1969. — La systématique des rongeurs hystricomorphes et la dérive des continents. Comptes rendus hebdomadaires des Séances de l'Académie des Sciences, série D, 269: 1496-1497. https://gallica.bnf.fr/ark:/12148/bpt6k63251842/f740.item

LAVOCAT R. 1973. - Les rongeurs du Miocène d'Afrique orientale. 1. Miocène inférieur. Mémoires et Travaux E.P.H.E. de l'Institut de Montpellier, $284 \mathrm{p}$.

LAVOCAT R. 1976. - Rongeurs caviomorphes de l'Oligocène de Bolivie. Rongeurs du bassin déséadien de Salla. Palaeovertebrata 7 (3): 15-90.

LEIDY J. 1871. - Notice of some extinct rodents. Proceedings of the Academy of Natural Sciences of Philadelphia 23: 230-232. https:// biodiversitylibrary.org/page/5512067

LESSON R.-P. 1842. - Nouveau tableau du Règne animal. Mammiferes. Arthus-Bertrand, Paris, $204 \mathrm{p}$

Li C. K., ZHENG J. J. \& Ting S. Y. 1989. - The skull of Cocomys lingchaensis, an early Eocene ctenodactyloid rodent of Asia, in BlaCK C. C. \& DaWsOn M. R. (eds), Papers on Fossil Rodents, in Honor of Albert Elmer Wood. Natural History Museum, Los Angeles: 179-192.

Livermore R., NANKIVEll A., EAgLES G. \& Morris P. 2005. — Paleogene opening of Drake Passage. Earth and Planetary Science Letters 236 (1): 459-470. https://doi.org/10.1016/j.epsl.2005.03.027

Loomis F. B. 1914. - The Deseado Formation of Patagonia. Rumford Press, Concord, New Hampshire, 232 p. https://doi.org/10.5962/ bhl.title. 28110

Macfadden B. J., Macfadden Jr. K. E., Cifelli R. L., Siles O. \& JoHnSON N. M. 1985. - Magnetic polarity stratigraphy and mammalian fauna of the Deseadan (Late Oligocene-Early Miocene) Salla beds of Northern Bolivia. The Journal of Geology 93: 223-250. https://doi.org/10.1086/628950

MADDISON W. P. \& MADDISON D. R. 2009. - Mesquite: a Modular System for Evolutionary Analysis. Version 2.72. University of British Columbia and Oregon State University.

Marivaux L. \& Welcomme J.-L. 2003. - New diatomyid and baluchimyine rodents from the Oligocene of Pakistan (Bugti Hills, Balochistan): systematic and paleobiogeographic implications. Journal of Vertebrate Paleontology 23: 420-434. https://doi.org/10.1671/0272-4634(2003)023[0420:NDA BRF]2.0.CO;2

Marivaux L., Welcomme J.-L., Vianey-Liaud M. \& Jaeger J.-J. 2002. - The role of Asia in the origin and diversification of hystricognathous rodents. Zoologica Scripta 31: 225-239. https:// doi.org/10.1046/j.1463-6409.2002.00074.x
Marivaux L., Vianey-Liaud M. \& Jaeger J.-J. 2004. — Highlevel phylogeny of early Tertiary rodents: dental evidence. Zoological Journal of the Linnean Society 142: 105-134. https://doi. org/10.1111/j.1096-3642.2004.00131.x

Marshall L. G. \& Sempéré T. 1993. - Evolution of the Neotropical Cenozoic land mammal fauna in its geochronologic, stratigraphic and tectonic context, in GOLDBLATT P. (ed.), Biological Relationships between Africa and South America. Yale University Press, New Haven: 329-392. https://doi. org/10.2307/j.ctt22726mc.16

MARTIN T. 1994. - On the systematic position of Chaetomys subspinosus (Rodentia: Caviomorpha) based on evidence from the incisor enamel microstructure. Journal of Mammalian Evolution 2 (2): 117-131. https://doi.org/10.1007/BF01464364

Martinod J., Husson L., Roperch P., Guillaume B. \& Espurt N. 2010. - Horizontal subduction zones, convergence velocity and the building of the Andes. Earth and Planetary Science Letters 299 (3-4): 299-309. https://doi.org/10.1016/j.epsl.2010.09.010

McGuire J. A., Witt C. C., Remsen J. V., Corl A., Rabosky D. L., Altshuler D. L. \& Dudley R. 2014. - Molecular phylogenetics and the diversification of hummingbirds. Current Biology 24 (8): 910-916. https://doi.org/10.1016/j.cub.2014.03.016

McKenna M. C. \& Bell S. K. 1997. - Classification of Mammals: Above the Species Level. Columbia University Press, New York, $631 \mathrm{p}$.

MÉGARD F. 1984. - The Andean orogenic period and its major structures in central and northern Peru. Journal of the Geological Society 141: 893-900. https://doi.org/10.1144/gsigs.141.5.0893

Meng J. \& McKenna M. C. 1998. - Faunal turnovers of Palaeogene mammals from the Mongolian Plateau. Nature 394 (6691): 364-367. https://doi.org/10.1038/28603

MENG J. \& WYSS A. R. 2001. - The morphology of Tribosphenomys (Rodentiaformes, Mammalia): phylogenetic implications for basal Glires. Journal of Mammalian Evolution 8: 1-70. https:// doi.org/10.1023/A:1011328616715

Meng J., Li C., Ni X., WANG Y. \& BeARD K. C. 2007a. A new Eocene rodent from the Lower Arshanto Formation in the Nuhetingboerhe (Camp Margetts) area, Inner Mongolia. American Museum Novitates 3569: 1-18. http://hdl.handle. net $/ 2246 / 5860$

MenG J., Li C., BEARD K. C., GEBO D. L., WANG Y. \& WANG H. 2007b. - New material of Alagomyidae (Mammalia, Glires) from the late Paleocene Subeng Locality, Inner Mongolia. American Museum Novitates 3597: 1-29. http://hdl.handle.net/2246/5893

Miller G. S. \& GiDLEY J. W. 1918. - Synopsis of the supergeneric groups of rodents. Journal of the Washington Academy of Sciences 8 (13): 431-448. https://doi.org/10.5962/bhl.part.6490

Miller K. G., Wright J. D. \& FairbanKs R. G. 1991. — Unlocking the ice house: Oligocene-Miocene oxygen isotopes, eustasy, and margin erosion. Journal of Geophysical Research: Solid Earth 96 (B4): 6829-6848. https://doi.org/10.1029/90JB02015

Mora A., Baby P., Roddaz M., Parra M., Brusset S., Hermoza W. \& Espurt N. 2010. - Tectonic history of the Andes and sub-Andean zones: implications for the development of the Amazon drainage basin, in HoORN C. \& WesseLINGH F. P. (eds), Amazonia, Landscape and Species Evolution: A Look into the Past. Blackwell-Wiley, Hoboken: 38-60.

Noble D., McKee E., Farrar E. \& Petersen U. 1974. - Episodic Cenozoic volcanism and tectonism in the Andes of Peru. Earth and Planetary Science Letters 21: 213-220. https://doi. org/10.1016/0012-821X(74)90057-0

Noble D., MéGARd F. \& MCKeE E. 1979. — Early tertiary “incaic” tectonism, uplift and volcanic activity, Andes of Central Peru. Bulletin of the Geological Society of America 90: 903-907. https:// doi.org/10.1130/0016-7606(1979)90<903:ETITUA>2.0.CO;2

NOVACEK M. J. 1993. - Patterns of diversity in the mammalian skull, in Hanken J. \& Hall B. K. (eds), The Skull. Volume 2. The University of Chicago Press, Chicago and London: 438-545. 
Olivares A. I. \& Verzi D. H. 2015. - Systematics, phylogeny and evolutionary pattern of the hystricognath rodent Eumysops (Echimyidae) from the Plio-Pleistocene of southern South America. Historical Biology 27 (8): 1042-1061. https://doi.org/10.10 80/08912963.2014.929672

Olivares A. I., Verzi D. H., Vucetich M.g. \& Montalvo C. I. 2012. Phylogenetic affinities of the late Miocene echimyid $†$ Pampamys and the age of Thrichomys (Rodentia, Hystricognathi). Journal of Mammalogy 93 (1): 76-86. https://doi.org/10.1644/11-MAMM-A-176.1

Olivero E. B., Barreda V., Marenssi S., Santillana S., MartiNIONI D. 1998. — Estratigrafía, sedimentología y palinología de la Formación Sloggett (Paleogeno continental), Tierra del Fuego. Revista de la Asociación Geológica Argentina 53: 504-516.

Oliveira F. B. De, Molina E. C. \& Marroig G. 2009. Paleogeography of the South Atlantic: a route for primates and rodents into the New World?, in GARBER P. A., EsTradA A., Bicca-Marques J. C., Heymann E. W. \& Strier K. B. (eds), South American Primates, Comparative Perspectives in the Study of Behavior, Ecology, and Conservation. Springer-Verlag, New York: 55-68. https://doi.org/10.1007/978-0-387-78705-3_3

Oncken O., Hindle D., Kley J., Elger P., Victor P. \& SChemMANN K. 2006. - Deformation of the central Andean upper plate system, facts, fiction, and constraints for plateau models, in Oncken O., Chong G., Franz G., Giese P., Götze H.-J., Ramos V. A., Strecker M. R. \& Wigger P. (eds), The Andes, Active Subduction Orogeny. Springer-Verlag, Berlin: 3-28. https:/ doi.org/10.1007/978-3-540-48684-8_1

Orliac M. J., ANTOINE P.-O. \& DuCrocQ S. 2010. — Phylogenetic relationships of the Suidae (Mammalia, Cetartiodactyla): new insights on the relations within Suoidea. Zoologica Scripta 39: 315-330. https://doi.org/10.1111/j.1463-6409.2010.00431.x

Page R. D. M. 2001. - NDE (Nexus Data Editor for Windows). Version 0.5.0 NDE. (University of Glasgow).

Palllas P. S. 1766. - Miscellanea Zoologica quibus novae imprimis atque obscurae animalium species describuntur et observationibus iconibusque illustrantur. Peter van Cleef, The Hague, 224 p. https://doi.org/10.5962/bhl.title.69851

PALAZZESI L. \& BARREDA V. - 2012. Fossil pollen records reveal a late rise of open-habitat ecosystems in Patagonia. Nature Communications 3: 1294. https://doi.org/10.1038/ncomms2299

Patterson B. \& PAscual R. 1968. - New echimyid rodents from the Oligocene of Patagonia, and a synopsis of the family. Breviora 301: 1-14. https://biodiversitylibrary.org/page/4294618

PATTERSON B. \& WoOD A. E. 1982. — Rodents from the Deseadan Oligocene of Bolivia and the relationships of the Caviomorpha. Bulletin of the Museum of Comparative Zoology 149 (7): 371-543. http://www.biodiversitylibrary.org/page/4776973

Patton J. L., Pardiñas U. F. \& Elía G. D’ (eds). 2015. - Mammals of South America. Volume 2: Rodents. University of Chicago Press, 384 p. http://ref.scielo.org/pyk23s

PÉrez M. E. 2010a. - A new rodent (Cavioidea, Hystricognathi) from the middle Miocene of Patagonia, mandibular homologies, and the origin of the crown group Cavioidea sensu stricto. Journal of Vertebrate Paleontology 30 (6): 1848-1859. https://doi.org/10. $1080 / 02724634.2010 .522432$

PÉrez M. E. 2010b. - Sistemática, ecología y bioestratigrafía de Eocardiidae (Rodentia, Hystricognathi, Cavioidea) del Mioceno temprano y medio de Patagonia. Volume de Thèse non publié, Universidad Nacional de La Plata, Argentina, 351 p.

Pérez M. E. \& Pol D. 2012. - Major radiations in the evolution of caviid rodents: reconciling fossils, ghost lineages, and relaxed molecular clocks. PLoS One 7 (10): e48380. https://doi. org/10.1371/journal.pone.0048380

Pérez M. E. \& Vucetich M. G. 2011. - A new extinct genus of Cavioidea (Rodentia, Hystricognathi) from the Miocene of Patagonia (Argentina), and the evolution of cavioid mandibular morphology. Journal of Mammalian Evolution 18 (3): 163-183. https://doi.org/10.1007/s10914-011-9154-1
Pérez M. E. \& Vucetich M. G. 2012a. - A revision of the fossil genus Phanomys Ameghino, 1887 (Rodentia, Hystricognathi, Cavioidea) from the early Miocene of Patagonia (Argentina) and the acquisition of euhypsodonty in Cavioidea sensu stricto. Paläontologische Zeitschrift 86 (2): 187-204. https://doi.org/10.1007/ s12542-011-0120-2

Pérez M. E. \& Vucetich M. G. 2012b. - Asteromys punctus Ameghino (Rodentia, Hystricognathi, Cavioidea) from the Late Oligocene of Patagonia (Argentina) and the early evolution of Cavioidea sensu stricto. Ameghiniana 49 (1): 118-125. https:// doi.org/10.5710/AMGH.v49i1(449)

Pérez M. E., Vucetich M. G. \& Kramarz A. G. 2010. - The first Eocardiidae (Rodentia) in the Colhuehuapian (early Miocene) of Bryn Gwyn (northern Chubut, Argentina) and the early evolution of the peculiar cavioid rodents. Journal of Vertebrate Paleontology 30 (2): 528-534. https://doi. org/10.1080/02724631003618223

Pérez M. E., Krause M. \& Vucetich M. G. 2012. - A new species of Chubutomys (Rodentia, Hystricognathi) from the late Oligocene of Patagonia and its implications on the early evolutionary history of Cavioidea sensu stricto. Geobios 45: 573-580. https://doi.org/10.1016/j.geobios.2012.06.001

Pérez M. E., Arnal M., Boivin M., Vucetich M. G., Candela A., Busker F. \& MAMani Quispe B. 2018. - New caviomorph rodents from the late Oligocene of Salla, Bolivia: taxonomic, chronological, and biogeographic implications in the Deseadan faunas of South America. Journal of Systematic Palaeontology. https://doi.org/10.1080/14772019.2018.1471622

PLEIJEL F. 1995. - On character coding for phylogeny reconstruction. Cladistics 11 (3): 309-315. https://doi.org/10.1016/07483007(95)90018-7

Pujos F. \& DE IuliIs G. 2007. — Late Oligocene Megatherioidea fauna (Mammalia: Xenarthra) from Salla-Luribay (Bolivia): New data on basal sloth radiation and Cingulata-Tardigrada Split. Journal of Vertebrate Paleontology 27: 132-144. https://doi. org/10.1671/0272-4634(2007)27[132:LOMFMX]2.0.CO;2

Prothero D. R. \& Berggren W. A. (eds) 1992. — Eocene-Oligocene Climatic and Biotic Evolution. Princeton University Press, Princeton, 568 p. https://www.jstor.org/stable/j.ctt7zvp65

Rambaut A. 2014. - FigTree. Version 1.4.2. Institute of Evolutionary Biology, University of Edinburgh.

RAsia L. L. \& CANDELA A. M. 2017. - Reappraisal of the giant caviomorph rodent Phoberomys burmeisteri (Ameghino, 1886) from the late Miocene of northeastern Argentina, and the phylogeny and diversity of Neoepiblemidae. Historical Biology. https:// doi.org/10.1080/08912963.2017.1294168

Reguero M. A. \& Candela A. M. 2001. - Late Cenozoic mammals from the northwest of Argentina, in SALFITY J. A. \& Marquillas R. A. (eds), Cenozoic Geology of the Central Andes of Argentina. SCS Publisher, Salta: 411-426.

Reguero M. A. \& Cerdeño E. 2005. - New late Oligocene Hegetotheriidae (Mammalia, Notoungulata) from Salla, Bolivia. Journal of Vertebrate Paleontology 25 (3): 674-684. https://doi. org/10.1671/0272-4634(2005)025[0674:NLOHMN]2.0.CO;2

Roddaz M., Hermoza W., Mora A., Baby P., Parra M., ChrisTOPHOUl F., BRusset S. \& Wesselingh F. P. 2010. — Cenozoic sedimentary evolution of the Amazonian foreland basin system, in HoORn C. \& Wesselingh F. P. (eds). Amazonia, Landscape and Species Evolution: A Look into the Past. Blackwell-Wiley, Hoboken: 61-88.

Roperch P., Sempéré T., Macedo O., Arriagada C., Fornari M., TAPIA C., GARCIA M. \& LAJ C. 2006. - Counterclockwise rotation of late Eocene-Oligocene fore-arc deposits in southern Peru and its significance for oroclinal bending in the central Andes. Tectonics 25 (3): TC3010. https://doi.org/10.1029/2005TC001882

Rovereto E. 1914. - Los estratos Araucanos y sus fósiles. Anales del Museo Nacional de Historia Natural de Buenos Aires 25: 1-247. http://www.biodiversitylibrary.org/page/14606247 
Sallam H. M. \& Seiffert E. R. 2016. - New phiomorph rodents from the latest Eocene of Egypt, and the impact of Bayesian "clock"-based phylogenetic methods on estimates of basal hystricognath relationships and biochronology. PeerJ 4: e1717. https://doi.org/10.7717/peerj.1717

Sallam H. M., Seiffert E. R., Steiper M. E. \& Simons E. L. 2009. - Fossil and molecular evidence constrain scenarios for the early evolutionary and biogeographic history of hystricognathous rodents. Proceedings of the National Academy of Sciences 106 (39): 16722-16727. https://doi.org/10.1073/ pnas.0908702106

Sallam H. M., Seiffert E. R. \& Simons E. L. 2011. — Craniodental morphology and systematics of a new family of hystricognathous rodents (Gaudeamuridae) from the Late Eocene and Early Oligocene of Egypt. PLoS One 6 (2): e16525. https:// doi.org/10.1371/journal.pone.0016525

Sallam H. M., Seiffert E. R. \& Simons E. L. 2012. — A basal phiomorph (Rodentia, Hystricognathi) from the late Eocene of the Fayum Depression, Egypt. Swiss Journal of Palaeontology 131 (2): 283-301. https://doi.org/10.1007/s13358-012-0039-6

Sánchez-Villagra M. R., Aguilera O. A. \& Horovirz J. 2003. - The anatomy of the world's largest extinct rodent. Science 301: 1708-1710. https://doi.org/10.1126/science.1089332

SCHAUB S. 1953. - Remarks on the distribution and classification of the Hystricomorpha. Verhandlungen der Naturforschenden Gesellschaft, Basel 64 (2): 389-400.

SCHAub S. 1958. - Simplicidentata (Rodentia), in Piveteau J. (ed), Traité de Paléontologie. Masson, Paris, 6 (2): 659-818.

ScOTT W. B. 1905. - Mammalia of the Santa Cruz beds. Volume V, Paleontology. Part III, Glires, in ScOTT W. B. (ed), Reports of the Princeton University Expeditions to Patagonia, 1896-1899. Princeton University, E. Schweizerbart'sche Verlaghandlung (E. Nägele), Stuttgart: 384-491. https://biodiversitylibrary. org/page/23542343

Shockey B. J., Hitz R. \& Bond M. 2004. — Paleogene notoungulates from the Amazon Basin of Peru, in CAMPBELl K. E. (ed.), The Paleogene Mammalian Fauna of Santa Rosa, Amazonian Peru. Natural History Museum of Los Angeles County, Science Series 40: 61-70.

Shockey B. J., Salas-Gismondi R., Gans P. B., Jeong A. \& FLYNN J. J. 2009. - Paleontology and geochronology of the Deseadan (late Oligocene) of Moquegua, Peru. American Museum Novitates 3668: 1-24. https://doi.org/10.1206/662.1

Sijp W. P. \& England M. H. 2004. - Effect of the Drake Passage through flow on global climate. Journal of Physical Oceanography 34 (5): 1254-1266. https://doi.org/10.1175/1520-048 5(2004)034<1254:EOTDPT>2.0.CO;2

SIMPSON G. G. 1945. - The principles of classification and a classification of mammals. Bulletin of the American Museum of Natural History 85: 1-350. http://hdl.handle.net/2246/1104

Springer M. S., Teeling E., Madsen O., Stanhope M. J. \& JONG W. W. DE 2001. - Integrated fossil and molecular data reconstruct bat echolocation. Proceedings of the National Academy of Sciences 98: 6241-6246. https://doi.org/10.1073/ pnas. 111551998

STEHLIN H. G. 1909. - Remarques sur les faunules de mammifères des couches éocènes et oligocènes du Bassin de Paris. Bulletin de la Société géologique de France 9: 488-520.

Steinmann G. 1929. - Geologie von Peru. Karl Winter, Heidelberg, $448 \mathrm{p}$.

StrömberG C. A., Dunn R. E., Madden R. H., Kohn M. J. \& CARLINI A. A. 2013. - Decoupling the spread of grasslands from the evolution of grazer-type herbivores in South America. Nature Communications 4 (1478): 1-8. https://doi. org/10.1038/ncomms 2508

Swofford D. L. 2002. - PAUP*. Phylogenetic Analysis Using Parsimony (*and Other Methods). Version 4. Sinauer Associates, Sunderland, Massachusetts.
Tejada-lara J., Salas-Gismondi R., Pujos F., Baby P., Benammi M., Brusset S., Franceschi D. De, Espurt N., Urbina M. \& ANTOINE P.-O. 2015. - Life in protoamazonia: Middle Miocene mammals from the Fitzcarrald Arch (Peruvian Amazonia). Palaeontology 58 (2): 341-378. https://doi. org/10.1111/pala.12147

THOMAS O. 1897. - On the genera of rodents: an attempt to bring up to date the current arrangement of the Order. Proceedings of the Zoological Society of London (part IV): 1012-1028. https:// doi.org/10.1111/j.1096-3642.1896.tb03097.x

TullberG T. 1899. - Üeber das System der Nagethiere, eine phylogenetische Studie. Nova Acta Regiae Societatis Scientarium Upsaliensis, serie 3, 18: 1-514. https://biodiversitylibrary.org/ page 14565643

UPHAM N. S. \& PATTERSON B. D. 2015. - Phylogeny and evolution of caviomorph rodents: a complete timetree for living genera, in VASSAllo A. I. \& ANTENUCCI D. (eds), Biology of Caviomorph Rodents: Diversity and Evolution. Sociedad Argentina para el Estudio de los Mamíferos (SAREM), Buenos Aires, Argentina: 63-120.

VerZI D. H. 1999. - The dental evidence on the differentiation of the ctenomyine rodents (Caviomorpha, Octodontidae, Ctenomyinae). Acta Theriologica 44 (3): 263-282. https://doi org/10.4098/AT.arch.99-25

Verzi D. H., Montalvo C. I. \& Vucetich M. G. 1991. — Nuevos restos de Xenodontomys simpsoni Kraglievich y la sistemática de los más antiguos Ctenomyinae (Rodentia, Octodontidae). Ameghiniana 28: 325-331.

Verzi D. H., Vucetich M. G. \& Montalvo C. I. 1995. - Un nuevo Eumysopinae (Rodentia, Echimyidae) del Mioceno tardío de la provincia de La Pampa y consideraciones sobre la historia de la subfamilia. Ameghiniana 32 (2): 191-195.

Verzi D. H., Montalvo C. I. \& Vucetich M. G. 1999. — Afinidades y significado evolutivo de Neophanomys biplicatus (Rodentia, Octodontidae) del Mioceno tardío-Plioceno temprano de Argentina. Ameghiniana 36 (1): 83-90.

Verzi D. H., Montalvo C. I. \& Tiranti S. I. 2003. - Un nuevo Xenodontomys (Rodentia, Octodontidae) del Mioceno tardío de La Pampa, Argentina. Patrón evolutivo y biocronología. Ameghiniand 40 (2): 229-238.

Verzi D. H., Vieytes E. C. \& Montalvo C. I. 2011. - Dental evolution in Neophanomys (Rodentia, Octodontidae) from the late Miocene of central Argentina. Geobios 44 (6): 621-633. https://doi.org/10.1016/j.geobios.2011.02.008

Verzi D. H., Olivares A. I. \& Morgan C. C. 2014. — Phylogeny and evolutionary patterns of South American octodontoid rodents. Acta Palaeontologica Polonica 59: 757-769. https://doi. org/10.4202/app.2012.0135

Verzi D. H., Olivares A. I., Morgan C. C. \& Álvarez A. 2016. Contrasting phylogenetic and diversity patterns in octodontoid rodents and a new definition of the family Abrocomidae. Journal of Mammalian Evolution 23 (1): 93-115. https://doi.org/10.1007/ s10914-015-9301-1

Vizcaíno S. F., Kay R. F. \& BARgo M. S. (eds) 2012. - Early Miocene Paleobiology in Patagonia: High-Latitude Paleocommunities of the Santa Cruz Formation. Cambridge University Press, Cambridge, 378 p. https://doi.org/10.1017/CBO9780511667381

VONHOF H. B. \& KAANDORP R. J. 2010. — Climate variation in Amazonia during the Neogene and the Quaternary, in Hoorn C. \& Wesselingh F. P. (eds), Amazonia, Landscape and Species Evolution: A Look into the Past. Blackwell-Wiley, Hoboken: 199-210.

Voss R. S., Hubbard C. \& Jansa S. A. 2013. - Phylogenetic relationships of New World porcupines (Rodentia, Erethizontidae): implications for taxonomy, morphological evolution, and biogeography. American Museum Novitates 3769: 1-36. https:// doi.org/10.1206/3769.2

VuCETiCH M. G. 1984. — Los roedores de la Edad Friasense (Mioceno Medio) de Patagonia. Revista del Museo de La Plata (Nueva Serie) 8 Paleontología 50: 47-126. 
Vucetich M. G. 1985. - Cephalomyopsis hipselodontus gen. et sp. nov. (Rodentia, Caviomorpha, Cephalomyidae) de la Edad Colhuehuapense (Oligoceno tardío) de Chubut, Argentina. Ameghiniana 22: 243-245.

VuCETICH M. G. 1989. — Rodents (Mammalia) of the Lacayani fauna revisited (Deseadan, Bolivia). Comparison with new Chinchillidae and Cephalomyidae from Argentina. Bulletin du Muséum national d'Histoire naturelle de Paris, série 4, 11, section C, 4: 233-247. https://biodiversitylibrary.org/page/55756950

VucETich M. G. \& BOND M. 1984. - Un nuevo Octodontoidea (Rodentia, Caviomorpha) del Oligoceno tardío de la provincia de Chubut (Argentina). Ameghiniana 21 (1): 105-114.

Vucetich M. G. \& Kramarz A. G. 2003. - New Miocene rodents from Patagonia (Argentina) and their bearing on the early radiation of the octodontoids (Hystricognathi). Journal of Vertebrate Paleontology 23: 435-444. https://doi. org/10.1671/0272-4634(2003)023[0435:NMRFPA]2.0.CO;2

Vucetich M. G. \& Ribeiro A. M. 2003. - A new and primitive rodent from the Tremembé Formation (Late Oligocene) of Brazil, with comments on the morphology of the lower premolars of caviomorph rodents. Revisa Brasileira de Paleontologia 5: 73-82.

Vucetich M. G. \& Verzi D. H. 1991. - Un nuevo Echimyidae (Rodentia, Hystricognathi) de la edad Colhuehuapense de Patagonia y consideraciones sobre la sistemática de la familia. Ameghiniana 28 (1-2): 67-74.

Vucetich M. G. \& Verzi D. H. 1994. - The presence of Protadelphomys (Rodentia, Echimyidae) in the Colhuehuapian of the South Barrancas of Lake Colhue Huapi (Chubut). Ameghiniana 31 (1): 93-94.

Vucetich M. G. \& Verzi D. H. 1996. — A peculiar octodontoid (Rodentia, Caviomorpha) with terraced molars from the Lower Miocene of Patagonia (Argentina). Journal of Vertebrate Paleontology 16 (2): 297-302. https://doi.org/10.1080/027246 34.1996.10011317

Vucetich M. G. \& Verzi D. H. 1999. — Changes in diversity and distribution of the caviomorph rodents during the Late Cenozoic in Southern South America. Quaternary of South America and Antarctic Peninsula 12: 207-223.

VuceTich M. G. \& Verzi D. H. 2002. — First record of Dasyproctidae (Rodentia) in the Pleistocene of Argentina: paleoclimatic implications. Palaeogeography, Palaeoclimatology, Palaeoecology 178: 67-73. https://doi.org/10.1016/S0031-0182(01)00402-3

Vucetich M. G. \& Vieytes E. C. 2006. — A middle Miocene primitive octodontoid rodent and its bearing on the early evolutionary history of the Octodontoidea. Palaeontographica Abteilung A 277 (1-6): 81-91.

Vucetich M. G., Verzi D. H. \& Dozo M. T. 1992. — El 'status' sistemático de Gaimanomys alwinea (Rodentia, Caviomorpha, Echimyidae). Ameghiniana 29 (1): 85-86.

Vucetich M. G., Mazzoni M. M. \& Pardiñas U. F. J. 1993a. Los roedores de la Formación Collón Cura (Mioceno Medio), y la ignimbrita Pilcaniyeu. Cañadón del Tordillo, Neuquen. Ameghiniana 30 (4): 361-381.

Vucetich M. G., Souza Cunha F. L. De \& Alvarenga H. M. F. DE 1993b. - Un Roedor Caviomorpha de la Formación Tremembé (Cuenca de Taubaté), Estado de Sao Paulo, Brasil. Anais da Academia Brasileira de Ciências 65 (3): 247-251.

Vucetich M. G., Verzi D. H. \& Hartenberger J.-L. 1999. Review and analysis of the radiation of the South American Hystricognathi (Mammalia, Rodentia). Comptes Rendus de l'Académie des Sciences - Series IIA - Earth and Planetary Science 329: 763-769. https://doi.org/10.1016/S1251-8050(00)88497-9

Vucetich M. G., Deschamps C. M., Olivares A. I. \& Dozo M. T. 2005. - Capybaras, size, shape, and time: a model kit. Acta Palaeontologica Polonica 50 (2): 259-272.

Vucetich M. G., Kramarz A. G. \& Candela A. M. 2010a. Colhuehuapian rodents from Gran Barranca and other Patagonian localities: the state of the art, in MADDEN R. H., CARLINI A. A.,
Vucetich M. G. \& KAY R. F. (eds), The Paleontology of Gran Barranca, Evolution and Environmental Change through the Middle Cenozoic of Patagonia. Cambridge University Press, Cambridge: 206-219.

Vucetich M. G., Vieytes E. C., Pérez M. E. \& Carlini A. A. 2010b. - The rodents from La Cantera and the early evolution of caviomorphs in South America, in MADDEN R. H., CARLINI A. A., Vucetich M. G. \& KAY R. F. (eds), The Paleontology of Gran Barranca, Evolution and Environmental Change through the Middle Cenozoic of Patagonia. Cambridge University Press, Cambridge: 189-201.

Vucetich M. G., Deschamps C. M. \& Pérez M. E. 2013. - Paleontology, evolution and systematics of capybara, in MoreIRA J., Ferraz K., Herrera E. \& Macdonald D. (eds), Capybara. Biology, Use and Conservation of an Exceptional Neotropical Species. Springer New York: 39-59. https://doi.org/10.1007/978-1-4614-4000-0_2 Vucetich M. G., Pérez M. E., Ciancio M. R., Carlini A. A., Madden R. H. \& Kohn M. J. 2014. - A new acaremyid rodent (Caviomorpha, Octodontoidea) from Scarritt Pocket, Deseadan (late Oligocene) of Patagonia (Argentina). Journal of Vertebrate Paleontology 34 (3): 689-698. https://doi.org/10.108 0/02724634.2013.804414

Vucetich M. G., Arnal M., Deschamps C. M., Pérez M. E. \& VIEYTES E. C. 2015a. - A brief history of caviomorph rodents as told by the fossil record, in VASSALlO A. I. \& ANTENUCCI D. (eds), Biology of Caviomorph Rodents: Diversity and Evolution. Sociedad Argentina para el Estudio de los Mamíferos (SAREM), Buenos Aires, Argentina: 11-62.

Vucetich M. G., Dozo M. T., Arnal M. \& Pérez M. E. 2015b. New rodents (Mammalia) from the Late Oligocene of Cabeza Blanca (Chubut) and the first rodent radiation in Patagonia. Historical Biology 27: 236-257. https://doi.org/10.1080/0891 2963.2014.883506

WAHLERT J. H. 1985. - Cranial foramina of rodents, in LUCKETT W. P. \& HARTENBERGER J.-L. (eds), Evolutionary Relationships among Rodents, a Multidisciplinary Analysis. Springer US, New York: 311-332. https://doi.org/10.1007/978-1-4899-0539-0_11 Walton A. H. 1990. - Rodents of the La Venta Fauna, Miocene, Colombia: biostratigraphy and paleoenvironmental implications. Unpublished Ph. D. Thesis, Southern Methodist University, Dallas, United States.

Walton A. H. 1997. - Rodents, in Kay R. F., Madden R. H., Cifelli R. L. \& Flynn J. J. (eds), Vertebrate Paleontology in the Neotropics. The Miocene Fauna of La Venta, Colombia. Smithsonian Institution Press, Washington and London: 392-409.

Waterhouse G. R. 1839. - Observations on the Rodentia, with a view to point out groups as indicated by the structure of the crania in this order of mammals. Magazine of Natural History 3: 90-96. https://biodiversitylibrary.org/page/2269965

Watrous L. E. \& WheEler Q. D. 1981. - The out-group comparison method of character analysis. Systematic Biology 30 (1): 1-11. https://doi.org/10.1093/sysbio/30.1.1

Wesselingh F. P., HoOrn C., KroOnenberg S. B., ANTONelli A., LundBerg J. G., Vonhof H. B. \& HoOghiemstra H. 2010. On the origin of Amazonian landscapes and biodiversity: a synthesis, in HoOrn C. \& WesselingH F. P. (eds), Amazonia, Landscape and Species Evolution: A Look into the Past. BlackwellWiley, Hoboken: 419-431.

Wible J. R., Wang Y., Li C. \& Dawson M. R. 2005. - Cranial anatomy and relationships of a new ctenodactyloid (Mammalia, Rodentia) from the early Eocene of Hubei Province, China. Annals of Carnegie Museum 74 (2): 91-150. https://doi.org/10.2992/00974463(2005)74[91:CAAROA]2.0.CO;2

WIENS J. J. 1995. - Polymorphic characters in phylogenetic systematics. Systematic Biology 44 (4): 482-500. https://doi. org/10.1093/sysbio/44.4.482

WiENS J. J. \& SERVEDIO M. R. - 1997. Accuracy of phylogenetic analysis including and excluding polymorphic characters. Systematic Biology 46 (2): 332-345. https://doi.org/10.1093/sysbio/46.2.332 
Wood A. E. 1949. - A new Oligocene rodent genus from Patagonia. American Museum Novitates 1435: 1-54. http://hdl.handle. net $/ 2246 / 2350$

Wood A. E. 1955. - A revised classification of the rodents. Journal of Mammalogy 36 (2): 165-187. https://doi.org/10.2307/1375874

WOOD A. E. 1962. — The early Tertiary rodents of the family Paramyidae. Transactions of the American Philosophical Society 52: 1-261.

Wood A. E. 1968 (ed.). - The African Oligocene Rodentia, in Early Cenozoic Mammalian Faunas, Fayum Province, Egypt. Part II. Peabody Museum Bulletin 28: 23-105. https://biodiversitylibrary. org/page/10587957

Wood A. E. \& PATTERSOn B. 1959. - The rodents of the Deseadan Oligocene of Patagonia and the beginnings of South American rodent evolution. Bulletin of the Museum of Comparative Zoology 120: 281-428. http://www.biodiversitylibrary.org/page/4780786

WoOdS C. A. 1984. - Hystricognath rodents, in ANDERSON S. \& JONES J. K. Jr (eds), Orders and Families of Recent Mammals of the World. John Wiley and sons: 389-446.
Woods C. A. \& Kilpatrick C. W. 2005. — Infraorder Hystricognathi, in Wilson D. E. \& ReEder D. M. (eds), Mammal Species of the World: a Taxonomic and Geographic Reference. Johns Hopkins University Press. Baltimore, 3e edition: 1538-1600.

Wyss A. R., FlynN J. J., NOREll M. A., SWISHER C. C. III, CHARRier R., NovaCeK M. J. \& MCKenna M. C. 1993. — South America's earliest rodent and recognition of a new interval of mammalian evolution. Nature 365 434-437. https://doi. org/10.1038/365434a0

Zachos J., Pagani M., Sloan L., Thomas E. \& Billups K. 2001. - Trends, rhythms, and aberrations in global climate 65 Ma to present. Science 292 (5517): 686-693. https://doi. org/10.1126/science. 1059412

Zachos J. C., Dickens G. R. \& Zeebe R. E. 2008. - An early Cenozoic perspective on greenhouse warming and carbon-cycle dynamics? Nature 451: 279-283. https://doi.org/10.1038/ nature 06588 


\section{ANNEXES}

ANNEXE 1. - Liste des 513 caractères dentaires et crânio-mandibulaires utilisés pour les analyses cladistiques réalisées lors du présent travail. Nombreux de ces caractères sont issus de ceux proposés par L. Marivaux à l'occasion de l'analyse phylogénétique publiée par Antoine et al. (2012). Toutefois, des observations nouvelles et la prise en compte d'autres analyses cladistiques (Carvalho \& Salles 2004; Candela \& Rasia 2012; Pérez et al. 2012; Kramarz et al. 2013; Arnal \& Vucetich 2015a; Verzi et al. 2016), ont permis de modifier, d'agrémenter substantiellement, et ainsi d'élargir considérablement le corpus de données morphologiques de la matrice initiale, publiée en données supplémentaires par Antoine et al. (2012). Les astérisques * signalent les caractères ordonnés.

\section{GENERAL DENTAL CHARACTERS}

1. *Cusp(-id)s and loph(-id)s: (0) bunodont; (1) loph(-id) s present but low; (2) lophodont (highly crested).

2. Cusp(-id)s: (0) bulbous; (1) sharpened.

3. *Loph(-id)s (at a moderate degree of wear): (0) thin; (1) thick; (2) very thick; (3) massive (inflated).

4. ${ }^{*}$ Orientation of the transverse crests or lamines on upper molars: (0) labiolingually straight; (1) slightly oblique; (2) strongly oblique.

5. ${ }^{*}$ Orientation of the transverse cristids or lamines on lower molars: (0) labiolingually straight; (1) slightly oblique; (2) strongly oblique.

6. Furrow opposite to hypoflexus on upper teeth at an advanced degree of wear: (0) absent; (1) present.

It is neither mesoflexus nor paraflexus.

7. Transverse dentine crest located at the middle of each molar lobe: (0) absent; (1) present.

8. ${ }^{*}$ Cement (in young-adult and at late ontogenetic stages): (0) absent or slightly present; (1) moderately present; (2) strongly present.

9. ${ }^{*}$ Degree of hypsodonty (of upper teeth): (0) brachydont (HI below to 1 and presence of roots); (1) mesodont (HI approximately equal to 1 and presence of roots); (2) protohypsodont (HI above to 1 and presence of roots); (3) euhypsodont (HI above to 1 and absence of roots).

10. Substitution of the deciduous premolars: (0) substitution occurs; (1) the deciduous premolars are retained in adults.

11. Upper tooth rows: (0) parallel (or very slightly converging anteriorly); (1) converging anteriorly.

12. Degree of the anterior convergence of the upper rows: (0) weak; (1) strong.

13. Distribution of enamel of hypoflexus/id on molars (Kramarz et al. 2013): (0) homogenous (equal thickness between the leading edges and the trailing ones); (1) heterogenous (the leading edges are thicker than the trailing edges).

14. Importance of the enamel heterogeneity in molars: (0) weak; (1) strong.

15. Number of roots in upper molars: (0) three (a lingual one and two labial); (1) four.

16. Type of HSB of incisors: (0) pauciserial; (1) multiserial. It is assumed that taxa from the same genus present the same condition regarding enamel microstructure.

17. ${ }^{*} I P M$ arrangement for multiserial enamel: (0) parallel IPM; (1) acute IPM; (2) rectangular IPM.

It is assumed that taxa from the same genus present the same condition regarding enamel microstructure.
18. Enamel of upper and lower incisors: (0) colourless; (1) with colour.

19. * Lower incisor: (0) long, bottom alveolar sheath at level of posterior portion of $\mathrm{m} 3$; (1) short, bottom alveolar sheath at level of $\mathrm{m} 2$ or $\mathrm{m} 3$ but not reaching posterior portion of $\mathrm{m} 3$; (2) extremely short, bottom alveolar sheath at level of $\mathrm{m} 1$.

It is assumed that taxa from the same genus present the same condition.

20. *Incisors procumbency: (0) opisthodont; (1) orthodont; (2) proodont.

It is assumed that taxa from the same genus present the same condition.

\section{CHARACTER ON DP3}

21. Third upper deciduous premolar: (0) present; (1) absent. Characters on P4:

22. * Size of P4 (at a moderate degree of wear): (0) P4 > M1; (1) $\mathrm{P} 4=\mathrm{M} 1$; (2) $\mathrm{P} 4<\mathrm{M} 1$.

23. Anterocingulum (or anteroloph) on P4: (0) absent; (1) present.

24. Length of the anterocingulum on P4: (0) short; (1) long.

25. Height of the anterocingulum on P4: (0) low; (1) high.

26. Anterocingulum - protocone connection on P4: (0) absent; (1) present (via an anterostyle/anterior arm of paracone or not).

27. Anterocingulum - paracone connection on P4: (0) absent; (1) present (via a parastyle/anterior arm of paracone or not).

28. Posterior arm of the paracone on P4: (0) absent; (1) present.

29. Protoconule on P4: (0) present; (1) absent.

30. Outgrowth of the protocone on P4: (0) absent; (1) present.

31. Length of the outgrowth of the protocone on P4: (0) long (connection with the hypocone, $=$ neo-endoloph); (1) short (no connection with the hypocone).

32. Height of the neo-endoloph on P4: (0) low; (1) high.

33. Labial protoloph on P4: (0) absent; (1) present.

34. Length of the labial protoloph on P4: (0) long (connection with the protoconule/mure/lingual protoloph); (1) short (no connection with the protoconule/mure/ lingual protoloph).

35. Orientation of the labial protoloph on P4: (0) transverse; (1) oblique (mesiolabially directed); (2) labially transverse and lingually oblique (mesiolabially directed).

36. Lingual protoloph on P4: (0) present (non-taeniodont or pseudo-taeniodont); (1) absent (taeniodont).

37. Height of the lingual protoloph on P4: (0) low; (1) high. 
38. * Orientation of the lingual protoloph on P4: (0) labiomesially oriented; (1) transverse; (2) labiodistally oriented.

39. Mure on P4: (0) absent; (1) present.

40. Height of the mure on P4: (0) low; (1) high.

41. Metaconule on P4: (0) present; (1) absent.

42. *Development of the metaconule on P4: (0) small; (1) moderate; (2) inflated.

43. Mesostyle on P4: (0) absent; (1) present.

44. *Development of the mesostyle on P4: (0) small; (1) moderate; (2) strong.

45. Height of the mesostyle on P4: (0) low; (1) high.

46. *Anterior isolation of the mesostyle on P4: (0) isolated; (1) faintly connected to the paracone or its posterior arm; (2) strongly connected to the paracone or its posterior arm.

47. * Posterior isolation of the mesostyle on P4: (0) isolated; (1) faintly connected to the metacone or its anterior arm or posteroloph; (2) strongly connected to the metacone or its anterior arm or posteroloph.

48. Mesostyle and metacone twinning on P4: (0) absent; (1) present.

49. Anterior arm of the mesostyle on P4: (0) absent; (1) present.

50. Posterior arm of the mesostyle on P4: (0) absent; (1) present.

51. Third crest (mesolophule and/or mesoloph) on P4: (0) absent; (1) present.

52. Mesolophule on P4: (0) absent; (1) present.

53. Length of the mesolophule on P4: (0) short; (1) long (reaches the buccal side of the tooth).

54. Height of the mesolophule on P4: (0) low; (1) high.

55. Lingual connection of the mesolophule on P4: (0) connected to the metaconule; (1) connected to the AAH; (2) connected to the metaloph.

56. Mesoloph on P4: (0) absent; (1) present.

57. Lingual connection of the mesoloph on P4: (0) free (unconnected); (1) connected to the mesolophule; (2) connected to the posteroloph (via a spur or not).

58. Hypocone on P4: (0) absent; (1) present.

59. Anterior arm of the hypocone (AAH) on P4: (0) absent; (1) present.

60. Length of the AAH on P4: (0) short; (1) long.

61. Orientation of the AAH on P4: (0) oblique (mesiolabially oriented); (1) transverse; (2) longitudinal.

62. Pronounced AAH-mure alignment on P4: (0) absent; (1) present.

63. Tendency to protoloph-AAH-mure alignment on P4: (0) absent; (1) present.

64. Metacone on P4: (0) present; (1) absent.

65. Metacone-posteroloph connection on P4: (0) absent; (1) present.

66. Fusion of the metacone with the posteroloph on P4: (0) absent; (1) present.
67. Anterior arm of the metacone on P4: (0) absent; (1) present.

68. Metaloph on P4: (0) present; (1) absent.

69. Lingual connection of the metaloph on P4: (0) free (unconnected); (1) connected to the metaconule or to the PAMul; (2) connected to the hypocone or AAH; (3) connected to the posteroloph (via a spur or not); (4) connected to the mesolophule.

70. Lingual connection of the metaloph with the AAH (via the mesolophule or not) and with the posteroloph on P4: (0) always absent; (1) sometimes present.

71. Direct metaloph (or metaconule)-protocone connection on P4: (0) present; (1) absent.

72. Height of the posteroloph on P4: (0) low; (1) high.

\section{CHARACTERS ON DP4}

73. ${ }^{*}$ DP4 length/width proportions: (0) length < width; (1) length = width; (2) length $>$ width.

74. Labial pericingulum on DP4: (0) present; (1) absent.

75. Length of the anterocingulum (or anteroloph) on DP4: (0) short; (1) long.

76. *Height of the anterocingulum (or anteroloph) on DP4: (0) low; (1) moderate; (2) high.

77. Anterocingulum (or anteroloph)-protocone connection on DP4: (0) absent; (1) present (via an anterostyle or the anterior arm of the protocone or not).

78. Anterocingulum (or anteroloph)-paracone connection on DP4: (0) absent; (1) present (via a parastyle or the anterior arm of the paracone or not).

79. Neocrestule on the paraflexus from the parastyle on DP4: (0) absent; (1) present.

80. Outgrowth of the protocone on DP4: (0) absent; (1) present.

81. Length of the outgrowth of the protocone on DP4: (0) short; (1) long (reaches the hypocone).

82. Posterior arm of the paracone on DP4: (0) absent; (1) present.

83. Protoconule on DP4: (0) present; (1) absent.

84. Orientation of the labial protoloph on DP4: (0) transverse or slightly mesiolingually oriented; (1) oblique (mesiolabially directed); (2) labially transverse and lingually oblique (mesiolabially directed).

85. Lingual protoloph on DP4: (0) present (non-taeniodont or pseudo-taeniodont); (1) absent (taeniodont).

86. Height of the lingual protoloph: (0) low; (1) high.

87. *Orientation of the lingual protoloph on DP4: (0) labiomesially oriented; (1) transverse; (2) labiodistally oriented.

88. Mure on DP4: (0) absent; (1) present.

89. Lenght of the mure on DP4: (0) short; (1) long (complete).

90. Metaconule on DP4: (0) present; (1) absent. 
91. Development of the metaconule on DP4: (0) strong; (1) small.

92. Metaconule position on DP4: (0) distal; (1) displaced mesially (central).

93. Mesostyle on DP4: (0) absent (indistinct) or faintly distinct; (1) present.

94. Surface of the mesostyle on DP4: (0) small; (1) strong.

95. *Anterior isolation of the mesostyle on DP4: (0) isolated; (1) faintly connected to the paracone or its posterior arm; (2) strongly connected to the paracone or its posterior arm.

96. *Posterior isolation of the mesostyle on DP4: (0) isolated; (1) slightly connected to the metacone or its anterior arm or the posteroloph; (2) strongly connected to the metacone or its anterior arm or the posteroloph.

97. Anterior arm of the mesostyle on DP4: (0) absent; (1) present.

98. Posterior arm of the mesostyle on DP4: (0) absent; (1) present.

99. Third crest (mesolophule and/or mesoloph) on DP4: (0) absent; (1) present.

100. Mesoloph on DP4: (0) absent; (1) present.

101. Length of the mesoloph on DP4: (0) short; (1) long (reaches the AAH).

102. Lingual connection of the mesoloph on DP4: (0) free (unconnected); (1) connected to the mesolophule or to the AAH; (2) connected to the posteroloph (via a spur or not).

103. Mesolophule on DP4: (0) absent; (1) present.

104. Length of the mesolophule on DP4: (0) short; (1) long (reaches the labial side of the tooth).

105. Hypocone on DP4: (0) absent; (1) present.

106. Development of the hypocone on DP4: (0) small; (1) strong.

107. Anterior arm of the hypocone (AAH) on DP4: (0) absent; (1) present.

108. Connection of the AAH on DP4: (0) with the metaconule/mure; (1) double: with the metaconule and the posterior outgrowth of the protocone.

109. Pronounced AAH-mure alignment on DP4: (0) absent; (1) present.

110. Tendency protoloph-AAH-mure alignment on DP4: (0) absent; (1) present.

111. Metacone on DP4: (0) present; (1) absent or indistinct.

112. Development of the metacone on DP4: $(0)$ strong (as the paracone); (1) small.

113. Anterior arm of the metacone on DP4: (0) absent; (1) present.

114. *Metacone-posteroloph connection on DP4: (0) unconnected; (1) faintly connected; (2) strongly connected.

115. Metaloph on DP4: (0) absent; (1) present.

116. Posterior arm of the metaconule (PAMul) on DP4: (0) absent; (1) present.
117. Lingual spur from the posteroloph on DP4: (0) absent; (1) present.

118. Lingual connection of the metaloph on DP4: (0) free (unconnected); (1) connected to the metaconule or to the PAMul; (2) connected to the third crest; (3) connected to the posteroloph (via the spur or not).

119. Direct metaloph(-metaconule)-protocone connection on DP4: (0) present; (1) absent.

\section{CHARACTERS ON UPPER MOLARS}

120. *Size of M1/M2: (0) M1 < M2; (1) M1 = M2; (2) M1 $>\mathrm{M} 2$.

121. * Size of M3/M2: (0) M3 < M2; (1) M2 = M3; (2) M3 $>\mathrm{M} 2$.

122. M1 length/width proportions: (0) length < width; (1) length $=$ width or width $<$ length .

123. M2 length/width proportions: (0) length < width; (1) length $=$ width or width $<$ length .

124. Labial pericingulum on upper molars (M1-3): (0) absent; (1) present.

125. Parastyle on upper molars: (0) absent; (1) present.

126. Height the parastyle on upper molars: (0) low; (1) high.

127. Anterostyle on upper molars: (0) always absent; (1) sometimes present.

128. *Anterocingulum (or anteroloph) on upper molars: (0) low; (1) moderately high; (2) high.

129. Length of the anterocingulum (or anteroloph) on upper molars: (0) short; (1) long.

130. Anterocingulum (or anteroloph)-paracone (or parastyle) connection on M1-2: (0) absent (paraflexus labially open); (1) present (paraflexus labially close, = parafossette).

131. Anterocingulum (or anteroloph)-protocone connection on M1: (0) absent; (1) present.

132. Anterocingulum (or anteroloph)-protocone connection on M2: (0) absent; (1) present.

133. Lingual anterocingulum (or anteroloph) on M1-2: (0) absent; (1) present.

134. Anterior arm of the protocone (AAPr) on M1-2: (0) always absent; (1) sometimes present or always present.

135. *Protocone configuration on upper molars: (0) rounded, bulbous (cuspate); (1) oblique, slightly mesiolabiallydistolingually pinched; (2) oblique, strongly mesiolabially-distolingually pinched.

136. Outgrowth of the protocone on M1: (0) absent; (1) present.

137. Length of the outgrowth of the protocone on M1: (0) long (reaches the hypocone); (1) short.

138. Outgrowth of the protocone on M2: (0) absent; (1) present.

139. Length of the outgrowth of the protocone on M2: (0) long (reaches the hypocone); (1) short. 
140. Outgrowth of the protocone on M3: (0) absent; (1) present.

141. Length of the outgrowth of the protocone on M3: (0) long (reaches the hypocone); (1) short.

142. Posterior arm of the paracone on upper molars: (0) present; (1) absent.

143. Thickness of the posterior arm of the paracone on upper molars: (0) thin; (1) thick.

144. Protoconule on upper molars: (0) present; (1) absent.

145. Labial protoloph on upper molars: (0) present; (1) absent.

146. Orientation of the labial protoloph on M1-2: (0) transverse; (1) oblique (mesiolabially directed); (2) lingually oblique and labially transverse.

147. Lingual protoloph on M1-2: (0) present (non-taeniodont or pseudo-taeniodont); (1) absent (taeniodont).

148. *Height of the lingual protoloph on M1-2: (0) very low (appears with wear); (1) low; (2) high.

149. Length of the lingual protoloph on M1-2: (0) long (complete); (1) short.

150. * Orientation of the lingual protoloph on M1-2: (0) labiomesially oriented; (1) transverse; (2) labiodistally oriented.

151. Lingual protoloph on M3: (0) present (non-taeniodont or pseudo-taeniodont); (1) absent (taeniodont).

152. *Height of the lingual protoloph on M3: (0) very low (appears with wear); (1) low; (2) high.

153. Length of the lingual protoloph on M3: (0) long (complete); (1) short.

154. * Orientation of the lingual protoloph on M3: (0) labiomesially oriented; (1) transverse; (2) labiodistally oriented.

155. Mure on M1-2: (0) absent; (1) present.

156. Length of the mure on M1-2: (0) short; (1) long (complete).

157. Mure on M3: (0) absent; (1) present.

158. Length of the mure on M3: (0) short; (1) long (complete).

159. *Height of the mure on upper molars: (0) very low (appears with wear); (1) low; (2) high.

160. Mure-protoloph connection on upper molars: (0) central; (1) more lingual.

161. Metaconule on upper molars: (0) present; (1) absent.

162. Development of the metaconule on upper molars: (0) strong; (1) small.

163. Metaconule position on upper molars: (0) distal or in line between the metacone and the protocone; (1) mesially displaced (central cusps).

164. Mesostyle on M1-2: (0) absent; (1) present.

165. Mesostyle on M3: (0) absent; (1) present.

166. *Development of the mesostyle on M1-2: (0) small; (1) moderate; (2) strong.

167. *Height of the mesostyle on M1-2: (0) low; (1) high; (2) very high (circa the height of the paracone).

168. *Position of the mesostyle on M1-2: (0) median; (1) distally displaced; (2) strongly distally displaced.
169. Anterior isolation of the mesostyle on M1-2: (0) isolated; (1) connected to the paracone or its posterior arm.

170. Anterior connection of the mesostyle on M1-2: (0) faintly connected to the paracone or its posterior arm; (1) strongly connected to the paracone or its posterior arm.

171. Posterior isolation of the mesostyle on M1-2: (0) isolated; (1) connected to the metacone or its anterior arm or posteroloph.

172. Posterior connection of the mesostyle on M1-2: (0) faintly connected to the metacone or its anterior arm or posteroloph; (1) strongly connected to the metacone or its anterior arm or posteroloph.

173. Anterior arm of the mesostyle on upper molars: (0) absent; (1) present.

174. Posterior arm of the mesostyle on upper molars: (0) absent; (1) present.

175. Third crest (mesolophule and/or mesoloph) on M1-2: (0) absent; (1) present.

176. Third crest (mesolophule andlor mesoloph) on M3: (0) absent; (1) present.

177. Mesolophule on M1-2: (0) absent; (1) present.

178. Mesolophule on M3: (0) absent; (1) present.

179. *Length of the mesolophule on upper molars: (0) very short (spur); (1) short; (2) long (reaches the labial side).

180. Mesoloph on M1-2: (0) absent; (1) present.

181. Mesoloph on M3: (0) absent; (1) present.

182. * Length of the mesoloph on upper molars: (0) very short (spur); (1) short; (2) long (reaches the AAH).

183. Lingual connection of the mesoloph on M1-2: (0) free (unconnected); (1) connected to the mesolophule; (2) connected to the AAH-mure; (3) connected to the metaloph; (4) connected to the posteroloph (via a spur or not); (5) connected to the hypocone.

The polymorphism corresponds here to the observation of different states on distinct specimens (for each of them) for a same taxon.

184. Double lingual connection of the mesoloph on M1-2: (0) absent; (1) present: with the mesolophule and the metaloph.

The double connection is scored (state 1) when a double connection is observed on one given specimen. The polymorphism corresponds here to the observation of different states on distinct specimens (for each of them) for a same taxon.

185. Lingual connection of the mesoloph on M3: (0) free (unconnected); (1) connected to the mesolophule; (2) connected to the AAH-mure; (3) connected to the metaloph; (4) connected to the posteroloph (via a spur or not).

The polymorphism corresponds here to the observation of different states on distinct specimens (for each of them) for a same taxon. 
186. Double lingual connection of the mesoloph on M3: (0) absent; (1) present: with the mesolophule and the metaloph; (2) present: with the mesolophule and the posteroloph (via a spur or not); (3) present: with the metaloph and the posteroloph.

The double connection is scored (states 1,2 or 3 ) when a double connection is observed on one given specimen. The polymorphism corresponds here to the observation of different states on distinct specimens (for each of them) for a same taxon.

187. Hypocone on M1-2: (0) absent; (1) present.

188. Hypocone on M3: (0) absent; (1) present.

189. Development of the hypocone on M1-2: (0) small; (1) strong.

190. Development of the hypocone on M3: (0) small; (1) strong.

191. Position of the hypocone with respect to the protocone on M1: (0) same level or more lingual; (1) more labial.

192. Position of the hypocone with respect to the protocone on M2: (0) same level or more lingual; (1) more labial.

193. Position of the hypocone with respect to the protocone on M3: (0) more labial (or same level); (1) strongly more labial.

194. Anterior arm of the hypocone (AAH) on M1-2: (0) absent; (1) present.

195. Connection of the AAH on M1: (0) with the metaconule/ mure; (1) double: with the metaconule/mure and the posterior outgrowth of the protocone.

196. Pronounced AAH-mure alignment on M1-2: (0) absent; (1) present.

197. Pronounced AAH-mure alignment on M3: (0) absent; (1) present.

198. Tendency protoloph-AAH-mure alignment on M1-2: (0) absent; (1) present.

199. Metacone on M1-2: (0) present; (1) absent.

200. Metacone on M3: (0) present; (1) absent.

201. *Development of the metacone on M2: (0) as large as the paracone; (1) reduced with respect to the paracone but distinct; (2) reduced with respect to the paracone and crestiform (not well-defined).

202. Development of the metacone on M3: (0) reduced with respect to the protocone but distinct; (1) reduced with respect to the protocone and crestiform (not welldefined).

203. * Position of the metacone with respect to the paracone on M2: (0) more labial; (1) mesiodistally aligned; (2) more lingual.

204. Anterior arm of the metacone on upper molars: (0) absent; (1) present.

205. Thickness of the anterior arm of the metacone on upper molars: (0) thin; (1) thick.

206. Posterior isolation of the metacone on M1-2: (0) isolated; (1) connected to the posteroloph (via its posterior arm or not).
207. Posteroloph-metacone connection on M1-2: (0) faintly connected; (1) strongly connected.

208. Posterior isolation of the metacone on M3: (0) isolated; (1) connected to the posteroloph (via its posterior arm or not).

209. Posteroloph-metacone connection on M3: (0) faintly connected; (1) strongly connected.

210. Mesostyle and metacone twinning on upper molars: (0) always absent; (1) sometimes present.

211. Metaloph on M1-2: (0) present; (1) absent.

212. Metaloph on M3: (0) present; (1) absent.

213. Height of the metaloph on upper molars: (0) very low (appears with the wear); (1) low; (2) high.

214. Posteroloph spur on M1-2: (0) absent; (1) present.

215. Lingual connection of the metaloph on M1-2: (0) free (unconnected); (1) connected to the metaconule; (2) connected to the third crest; (3) connected to the posteroloph (via the spur or not).

The polymorphism corresponds here to the observation of different states on distinct specimens (for each of them) for a same taxon.

216. Double lingual connection of the metaloph on M1-2: (0) absent; (1) with the metaconule and the posteroloph (via the spur or not); (2) with the third crest and the posteroloph (via the spur or not); (3) with the AAHmure and the posteroloph (via the spur or not).

The double connection is scored (states 1,2 or 3) when a double connection is observed on one given specimen. The polymorphism corresponds here to the observation of different states on distinct specimens (for each of them) for a same taxon.

217. Lingual connection of the metaloph on M3: (0) free (unconnected); (1) connected to the metaconule; (2) connected to the third crest; (3) connected to the AAHmure; (4) connected to the posteroloph (via the spur or not).

The polymorphism corresponds here to the observation of different states on distinct specimens (for each of them) for a same taxon.

218. Double lingual connection of the metaloph on M3: (0) absent; (1) with the metaconule and the posteroloph (via the spur or not); (2) with the third crest and the posteroloph (via the spur or not); (3) with the AAHmure and the posteroloph (via the spur or not).

The double connection is scored (states 1,2 or 3 ) when a double connection is observed on one given specimen. The polymorphism corresponds here to the observation of different states on distinct specimens (for each of them) for a same taxon.

219. Direct metaloph(-metaconule)-protocone connection on M1: (0) present; (1) absent.

220. Direct metaloph(-metaconule)-protocone connection on M2: (0) present; (1) absent. 
221. Labial part of the posteroloph on upper molars: (0) low; (1) high.

222. Lingual connection of the posteroloph on M1-2: (0) isolated or faintly connected to the hypocone; (1) strongly connected to the hypocone.

223. Lingual connection of the posteroloph on M3: (0) isolated or faintly connected to the hypocone; (1) strongly connected to the hypocone.

224. Size of the notch between the posteroloph and the hypocone on M1-2: (0) large; (1) small.

225. Size of the notch between the posteroloph and the hypocone on M3: (0) large; (1) small.

226. Paraflexus (+hypoflexus) on moderately worn M2: (0) present; (1) absent (with wear).

Degree of wear: P4 (when associated with M2) rather worn.

227. * Reduction of the paraflexus (+hypoflexus) on moderately worn M2: (0) weak; (1) moderate; (2) strong.

Degree of wear: P4 (when associated with M2) rather worn.

228. Mesial mesoflexus (+distal meso/posteroflexus) on moderately worn M2: (0) absent (with wear); (1) present.

Degree of wear: P4 (when associated with M2) rather worn.

229. * Reduction of the mesial mesoflexus (+distal meso posteroflexus) on moderately worn M2: (0) weak; (1) moderate; (2) strong.

Degree of wear: P4 (when associated with M2) rather worn.

230. Posteroflexus (labial part at least) on moderately worn M2: (0) present; (1) absent (with wear).

Degree of wear: P4 (when associated with M2) rather worn.

231. Reduction of the posteroflexus (labial part at least) on moderately worn M2: (0) weak; (1) strong.

Degree of wear: P4 (when associated with M2) rather worn.

232. Hypoflexus on moderately worn M2: (0) absent (with wear); (1) present.

Degree of wear: P4 (when associated with M2) rather worn.

233. Reduction of the hypoflexus on moderately worn M2: (0) weak; (1) strong.

Degree of wear: P4 (when associated with M2) rather worn.

234. *Transversal extension of the hypoflexus on moderately worn M1-2: (0) weak; (1) moderate; (2) strong.

235. Formation of lobe(s) on upper molars with wear: (0) absent; (1) present.

236. Number of lobes on M1-2: (0) one; (1) two.

237. *Number of lobes on M3: (0) one; (1) two; (2) three.

238. Complete fusion of lophs on upper molars: (0) absent; (1) present.

239. Fusion of the third crest with the posteroloph in a posterior lobe on upper molars with moderate wear: (0) absent; (1) present.
240. Fusion of the protoloph (a part or entire), the third crest and the posteroloph in a posterior lobe on upper molars with moderate wear: (0) absent; (1) present.

241. Shape of the anterior lobe of the M1-2: (0) heart-shaped; (1) laminar; (2) triangular; (3) suboval.

242. Shape of the posterior lobe of the M1-2: (0) heart-shaped; (1) laminar; (2) triangular; (3) suboval.

243. Posterior projection of the posterior lobe of M3: (0) absent; (1) present.

244. Lamines on pristine M1-2: (0) absent; (1) present.

245. Lamines on pristine M3: (0) absent; (1) present.

246. Lamines on worn M1-2: (0) absent; (1) present.

247. Lamines on worn M3: (0) absent; (1) present.

248. *Number of lamines on pristine or little worn M1-2: (0) three; (1) four; (2) five.

249. *Number of lamines on pristine or little worn M3: (0) three; (1) four; (2) five; (3) six; (4) more than ten.

250. Primary external flexus (H.P.E.) on M1-2: (0) absent; (1) present.

251. Secondary external flexus (H.S.E.) on M1-2: (0) absent; (1) present.

\section{REMARK}

The orientation of the labial protoloph on upper teeth (characters 35, 84 and 146) does not appear to evolve with respect to a morphocline. Based on our observations, this feature could be ontogenetic and linked to the wear in some taxa. This hypothesis needs to be validated. For the time being, we prefer to not ordinate these characters.

\section{CHARACTERS ON P4}

252. *Length of $\mathrm{p} 4 / \mathrm{m} 1$ : (0) $\mathrm{p} 4>\mathrm{m} 1$; (1) $\mathrm{p} 4=\mathrm{m} 1$; (2) $\mathrm{p} 4<$ $\mathrm{m} 1 ;$ (3) $\mathrm{p} 4<\mathrm{m} 1$.

At a medium degree of wear (in case of hypsodont teeth).

253. Width of 4 /m1: (0) $\mathrm{p} 4>$ or $=\mathrm{m} 1$; (1) $\mathrm{p} 4<\mathrm{m} 1$. At a medium degree of wear (in case of hypsodont teeth).

254. *Talonid of $p 4$ with respect to the trigonid: (0) narrower; (1) equal; (2) wider.

255. Anterior cingulid on $p 4:(0)$ absent; (1) present.

256. *Development of the metaconid with respect to the protoconid on $p 4$ : (0) metaconid > protoconid; (1) metaconid $=$ protoconid; $(2)$ metaconid $<$ protoconid.

257. Metaconid-protoconid proximity on $p 4$ : (0) widely spaced; (1) closely spaced.

258. *Position of the metaconid with respect to the protoconid on $p 4$ : (0) posterior; (1) aligned; (2) anterior.

259. Metalophulid I (AAP and/or AAM) on $p 4$ : (0) present; (1) absent.

260. Anterior arm of the metaconid (AAM) on p4: (0) present; (1) absent. 
261. Anterior arm of the protoconid (AAP) on $p 4$ : (0) present; (1) absent.

262. Labial connection of the anterior arm of the metaconid on p4: (0) free; (1) with the anterior arm of the protoconid; (2) with the protoconid; (3) with the anteroconid.

263. Posterior arm of the metaconid (PAM) on $p 4$ : (0) present; (1) absent.

264. Thickness of the PAM on p4: (0) thin; (1) thick.

265. Mesostylid on $p 4:(0)$ absent; (1) present.

266. *Anterior isolation of the mesostylid on $p 4:(0)$ isolated; (1) faintly connected to the metaconid or its posterior arm; (2) strongly connected to the metaconid or its posterior arm.

267. Buccal metaconid cristid/spur on $p 4$ : (0) absent; (1) present.

268. Neocristid on the anteroflexid (between the med. I and the second transverse cristid, stemming from the metaconid or its posterior arm) on $p 4:$ (0) always absent; (1) sometimes present.

269. Posterior arm of the protoconid (PAP) on $p 4:(0)$ present; (1) absent.

270. Lingual connection of the PAP on $p 4$ : (0) free; (1) connected to the metaconid or its vicinity (med II); (2) connected to the neomesolophid; (3) connected to the buccal metaconid spur; (4) connected to the mesolophid. The polymorphism corresponds here to the observation of different states on distinct specimens (for each of them) for a same taxon.

271. Double lingual connection of the PAP on $p 4$ : (0) absent; (1) with the buccal metaconid spur and the mesolophid; (2) with the neomesolophid and the mesolophid. The double connection is scored (states 1 or 2 ) when a double connection is observed on one given specimen. The polymorphism corresponds here to the observation of different states on distinct specimens (for each of them) for a same taxon.

272. Neomesolophid on 4 : (0) absent; (1) present.

273. Mesiolabial obliquity of the neomesolophid on $p 4:(0)$ absent; (1) present.

274. Intensity of the mesiolabial obliquity of the neomesolophid on p4: (0) weak; (1) strong.

275. Labial connection of the neomesolophid with the med. I or its vicinity on $p 4$ : (0) absent; (1) present.

276. Mesoconid on $p$ : (0) absent; (1) present.

277. Mesolophid on $p 4$ : (0) absent; (1) present.

278. Length of the mesolophid on $p 4:(0)$ short; (1) long (reaches the lingual margin of the tooth).

279. Ectolophid on p4: (0) absent; (1) present.

280. * Height of the ectolophid on $p 4:(0)$ very low (appears with wear); (1) low; (2) high.

281. Interruption of the ectolophid (in its middle) on p4: (0) absent; (1) present.

282. Notch between the ectolophid and the protoconid on $p 4$ : (0) absent; (1) present.
283. Development of the notch between the ectolophid and the protoconid on $p 4$ : (0) shallow; (1) deep.

284. * Posterior isolation of the entoconid (or its posterior arm) on 4 : (0) isolated; (1) faintly connected to the posterolophid; (2) strongly connected to the posterolophid.

285. Hypolophid on $p 4$ : (0) absent; (1) present.

286. Length of the hypolophid on $p 4$ : (0) long; (1) short (labial part absent).

287. Labial connection of the hypolophid on $p 4$ : (0) free; (1) with the posterolophid (/hypoconulid); (2) with the ectolophid/AAH; (3) double connection: with the AAH/ectolophid and the PAH/posterolophid.

288. Posterior cristid formed by the fusion of the hypolophid with the posterolophid on $p 4$ (at middle degree of wear): (0) absent; (1) present.

289. Alignment of the hypolophid and ectolophid on p4: (0) absent; (1) present.

290. Anterior arm of the hypoconid (AAH) on p4: (0) absent (taeniodont); (1) present (non-taeniodont or pseudotaeniodont).

291. Height of the AAH on $p 4$ : (0) low; (1) high.

292. Length of the AAH on $p 4$ : (0) long (reaches the ectlophid/ hypolophid); (1) short.

293. Anterior outgrowth of the hypoconid on $p 4$ : (0) absent; (1) present.

294. Hypoconulid on 4 : (0) absent; (1) present.

295. Development of the hypoconulid on $p 4:$ (0) small; (1) strong.

296. *Distance between the hypolophid and the posterior arm of the hypoconid (PAH)/posterolophid on p4: (0) welldistant; (1) close; (2) very close.

297. Anterior projection on $p 4$ : (0) absent; (1) present.

298. Development of the anterior projection on $p 4:(0)$ weak; (1) strong.

299. Primary internal flexid (h.p.i.) on p4: (0) absent; (1) present.

300. Fundamental external flexid (h.f.e.) on p4: (0) absent; (1) present.

301. Secondary external flexid (h.s.e.) on p4: (0) absent; (1) present.

\section{Characters on dp4:}

302. Proportions of $d p 4:(0)$ length $<$ or $=$ width; (1) length clearly $>$ width.

303. Trigonid width on dp4: (0) narrow; (1) wide.

304. Paraconid (or paraconide-like) on dp4: (0) present; (1) absent.

305. Anteroconid on dp4: (0) absent; (1) present.

306. Development of the anteroconid on dp4: (0) small; (1) large.

307. Anterior cingulid on dp4: (0) present; (1) absent.

308. * Position of the metaconid with respect of the protoconid on dp4: (0) posterior; (1) aligned; (2) anterior. 
309. Metaconid-protoconid proximity on dp4: (0) widely spaced or slightly close; (1) very close.

310. Metalophulid I (med. I) on dp4: (0) absent or incomplete; (1) complete.

In some African hystricognaths (e.g. Acritophiomys, Phiomys andrewsi and $P$. hammudai), the anteroconid (and often its anterior and posterior arms too) and/or the anterior cingulid can be connected to the med I (i.e., anterior arms of the protoconid and/or the metaconid). So, the anterior cristid in caviomorph dp4, coded here as a med. I, could be a more complex structure composed of a med. I, anteroconid and anterior cingulid.

311. Lingual connection of the med. I with the metaconid on dp4: (0) absent; (1) present.

312. Labial connection of the med. I with the protoconid on dp4: (0) absent; (1) present.

313. Mesostylid on dp4: (0) absent; (1) present.

314. Anterior isolation of the mesostylid on dp4: (0) isolated; (1) connected to the metaconid or its posterior arm.

315. Anterior connection of the mesostylid on $d p 4$ : (0) faintly connected to the metaconid or its posterior arm; (1) strongly connected to the metaconid or its posterior arm.

316. Posterior isolation of the mesostylid on $d p 4:(0)$ isolated; (1) connected to the entoconid or its anterior arm.

317. Metaconid cristid/spur on dp4: (0) absent; (1) present.

318. Neocristid 1 (between the med. I and the second transverse cristid, stemming from the lingual margin, distal to the metaconid) on dp4: (0) absent; (1) present.

319. Labial connection of the neocristid 1 on $d p 4:$ (0) free; (1) with the PAP.

320. Neocristid 2 (between the med. I and the second transverse cristid, mesial to the metaconid) on dp4: (0) absent; (1) present.

321. Labial connection of the neocristid 2 on dp 4 : (0) free; (1) with the med I.

322. Posterior arm of the protoconid (PAP) on dp4: (0) absent; (1) present.

323. Lingual connection of the PAP on dp4: (0) free; (1) connected to the metaconid or in its vicinity (= med. II); (2) connected to the mesolophid/neomesolophid; (3) connected to the metaconid cristid/spur; (4) connected to the mesostylid or in its vicinity.

The polymorphism corresponds here to the observation of different states on distinct specimens (for each of them) for a same taxon.

324. Double lingual connection of the PAP on dp4: (0) absent; (1) connected to the mesolophid/neomesolophid and to the metaconid cristid/spur (via secondary cristids or not); (2) connected to the mesolophid/neomesolophid and to the mesostylid or in its vicinity (via secondary cristids or not).
The double connection is scored (states 1 or 2 ) when a double connection is observed on one given specimen. The polymorphism corresponds here to the observation of different states on distinct specimens (for each of them) for a same taxon.

325. Protoconid cristid on dp4: (0) absent; (1) present.

326. Connection of the protoconid cristid with the mesoconid/ ectolophid on dp4: (0) present; (1) absent.

327. Third cristid (mesolophid and lor neomesolophid) on dp4: (0) absent; (1) present.

328. *Orientation of the third cristid on $d p 4:(0)$ transverse or distolingual; (1) mesiolingual; (2) very mesiolingual.

329. Neomesolophid on dp4: (0) absent; (1) present.

330. Length of the neomesolophid on dp4: (0) short (less or equal than the half of the width of the mesoflexus); (1) long (more than the half of the width of the mesoflexus).

331. Strong forward orientation of the labial extremity of the neomesolophid on dp4: (0) absent; (1) present.

332. Mesoconid on dp4: (0) present; (1) absent.

333. Mesolophid on dp4: (0) absent; (1) present.

334. Lingual connection of the mesolophid on $d p 4:(0)$ free; (1) connected to the mesostylid; (2) connected to the neomesolophid.

335. Mesial ectolophid on dp4: (0) absent; (1) present.

336. Orientation of the mesial ectolophid on $d p 4$ : (0) longitudinal; (1) mesiolabial and/or transverse.

337. Distal ectolophid on dp4: (0) absent; (1) present.

338. Alignment of the mesial and distal ectolophids on dp4: (0) absent; (1) present.

339. Mesoconid-like cuspid on the mesial ectolophid (labial) on dp4: (0) absent; (1) present.

340. *Position of the entoconid with respect of the hypoconid on dp4: (0) distal; (1) aligned; (2) mesial.

341. Posterior isolation of the entoconid on dp4: (0) isolated; (1) connected to the posterolophid.

342. Hypolophid on dp4: (0) absent; (1) present.

343. Alignment of the hypolophid with the ectolophid on dp4: (0) absent; (1) present.

344. Hypoconulid on dp4: (0) present; (1) absent.

345. Development of the hypoconulid on dp4: (0) small; (1) well-developed.

346. Anterior arm of the hypoconid (AAH) on dp4: (0) absent (taeniodont); (1) present (non-taeniodont or pseudotaeniodont).

347. *Height of the AAH on $d p 4:(0)$ very low (appears with wear); (1) low; (2) high.

348. Length of the AAH on dp4: (0) long (complete); (1) short.

349. Anteroflexid on moderately worn dp4: (0) present; (1) absent (with wear).

350. (Distal) mesoflexid on moderately worn dp4: (0) present; (1) absent (with wear).

351. Metaflexid on moderately worn dp4: (0) present; (1) absent (with wear). 


\section{CHARACTERS ON LOWER MOLARS}

352. *Trigonid height on lower molars: (0) clearly much higher than the talonid; (1) slightly higher than the talonid; (2) as low as the talonid.

353. Trigonid width of $m 2$ : (0) narrower than the talonid; (1) as wide as the talonid or slightly wider.

354. * Length of $\mathrm{m} 3 / \mathrm{m} 2$ : (0) $\mathrm{m} 3>\mathrm{m} 2$; (1) $\mathrm{m} 3=\mathrm{m} 2$; (2) $\mathrm{m} 3$ $<\mathrm{m} 2$.

355. *Distance between the distal extremity of the hypolophid (or mesial extremity of the $A A H)$ and the PAP (= length of the ectolophid when it is present) on $m 2$ : (0) long; (1) short; (2) very short.

356. Anterocingulid on lower molars: (0) absent; (1) present.

357. Position of the protoconid with respect to the metaconid on $m 2$ : (0) opposed or protoconid anterior; (1) protoconid posterior.

358. * Protoconid/metaconid height on lower molars: (0) metaconid clearly higher than the protoconid; (1) metaconid slightly higher than the protoconid; (2) metaconid as high as the protoconid or lower.

359. Posterior arm of the metaconid (PAM) on lower molars: (0) present: (1) absent.

360. Length of the PAM on lower molars: (0) long; (1) short.

361. Thickness of the PAM on lower molars: (0) thin; (1) thick.

362. Lingual connection of the metalophulid I (med. I) with the metaconid on lower molars: (0) present; (1) absent.

363. Labial connection of the med. I with the protoconid on lower molars: (0) present; (1) absent.

364. Height of the med. I on lower molars: (0) low; (1) high.

365. Interruption of the med. I on lower molars: (0) absent; (1) present.

366. Mesostylid on lower molars: (0) absent; (1) present.

367. Anterior isolation of the mesostylid on lower molars: (0) isolated; (1) connected to the metaconid or PAM.

368. Posterior isolation of the mesostylid on lower molars: (0) isolated; (1) connected to the entoconid or its anterior arm.

369. Metaconid cristid/spur on lower molars: (0) absent; (1) present.

370. Neocristid (between the med. I and the second transverse cristid, stemming from the lingual margin, distal to the metaconid) on lower molars; (0) absent; (1) present.

371. Posterior arm of the protoconid (PAP) on m1-2: (0) present; (1) absent.

372. * Length of the PAP on m1-2: (0) long (more than the half of the width of the first flexid); (1) short (less or equal than the half of the width of the first flexid); (2) very short (spur).

373. Lingual connection of the PAP on m1-2: (0) free; (1) connected to the metaconid or its vicinity; (2) connected to the neomesolophid/mesolophid; (3) connected to the mesostylid or its vicinity; (4) connected to the metaconid cristid/spur; (5) connected to the med. I (via secondary cristulids or not).
374. Posterior arm of the protoconid (PAP) on m3: (0) present; (1) absent.

375. *Length of the PAP on m3: (0) long (more than the half of the width of the first flexid); (1) short (less or equal than the half of the width of the first flexid); (2) very short (spur).

376. Lingual connection of the PAP on m3: (0) free; (1) connected to the metaconid or its vicinity; (2) connected to the neomesolophid/mesolophid; (3) connected to the mesostylid or its vicinity; (4) connected to the metaconid cristid/spur.

377. Height of the PAP on lower molars: (0) low; (1) high.

378. Direction of the PAP on lower molars: (0) directed toward the metaconid; (1) clearly oblique, extending backwardly.

379. Labial connection of the PAP on lower molars: (0) direct; (1) via the protoconid cristid.

380. Double lingual connection of the PAP on lower molars: (0) absent; (1) connected to the neomesolophid/mesolophid and to the metaconid crsitid/spur (via secondary cristulids or not).

381. Metalophular spur on lower molars: (0) absent; (1) present.

382. Protoconid cristid on lower molars: (0) present; (1) absent.

383. Neomesolophid on lower molars: (0) absent; (1) present.

384. Length of the neomesolophid on lower molars: (0) short (less or equal than the half of the width of the first flexid); (1) long (more than the half of the width of the first flexid).

385. Labial connection of the neomesolophid with the met. I on lower molars: (0) absent; (1) present.

386. Mesoconid on lower molars: (0) present; (1) absent.

387. Mesolophid on m1-2: (0) absent; (1) present.

388. Lingual connection of the mesolophid on m1-2: (0) free; (1) connected to the mesostylid; (2) connected to the neomesolophid; (3) connected to the PAP or to the metalophular spur.

389. Mesolophid on m3: (0) absent; (1) present.

390. Lingual connection of the mesolophid on m3: (0) free; (1) connected to the mesostylid; (2) connected to the neomesolophid.

391. Ectolophid on m1-2: (0) absent; (1) present.

392. Mesiolabial obliquity of the ectolophid on lower molars: (0) absent; (1) present.

393. Alignment of the ectolophid (at least the mesial one) with the protoconid cristid on lower molars: (0) absent; (1) present.

394. Mesiodistal pinch of the hypoconid on lower molars: (0) absent; (1) present.

395. Position of the entoconid with respect to the hypoconid on $m 2$ : (0) aligned or entoconid posterior; (1) entoconid anterior.

396. Anterior arm of the entoconid on lower molars: (0) absent; (1) present. 
397. Posterior isolation of the entoconid (or its posterior arm) on m1-2: (0) isolated; (1) connected to the posteroloph.

398. Hypolophid on lower molars: (0) absent; (1) present.

399. Height of the hypolophid on lower molars: (0) low; (1) high.

400. Length of the hypolophid on lower molars: (0) short; (1) long (complete, reaches the ectolophid/AAH).

401. Obliquity of the hypolophid on lower molars: (0) absent or slightly present; (1) strongly present.

402. Alignment of the hypolophid with the ectolophid on lower molars: (0) absent; (1) present.

403. Anterior arm of the hypoconid $(A A H)$ on lower molars: (0) present (non-taeniodont or pseudo-taeniodont); (1) absent (taeniodont).

404. *Height of the AAH on lower molars: (0) very low (appears with wear); (1) low; (2) high.

405. Length of the AAH on lower molars: (0) long (complete, reaches the ectolophid/mesoconid); (1) short.

406. Hypoconulid on m1-2: (0) present; (1) absent.

407. Development of the hypoconulid on m1-2: (0) strong; (1) small.

408. Hypoconulid on m3: (0) present; (1) absent.

409. Anteroflexid (or a part) on moderately worn lower molars: (0) present; (1) absent (with wear).

410. Strong reduction of the anteroflexid on moderately worn lower molars: (0) absent; (1) present.

411. Mesoflexid (+anteroflexid) on moderately worn lower molars: (0) present; (1) absent (with wear).

412. Strong reduction of the mesoflexid (+anteroflexid) on moderately worn lower molars: (0) absent; (1) present.

413. Metaflexid on moderately worn lower molars: (0) present; (1) absent (with wear).

414. Strong reduction of the metaflexid on moderately worn lower molars: (0) absent; (1) present.

415. Hypoflexid on moderately worn lower molars: (0) present; (1) absent (with wear).

416. Transversal extension of the hypoflexid on moderately worn m1-2: (0) absent; (1) present (extending at the transverse midpoint of the crown or beyond).

417. Importance of the transversal extension of the hypoflexid on moderately worn m1-2: (0) weak (extending at the transverse midpoint of the crown); (1) strong (extending beyond the transverse midpoint of the crown).

418. Formation of lobe(s) with wear on lower molars: (0) absent; (1) present.

419. Number of lobes on lower molars: (0) one; (1) two.

420. Shape of the anterior lobe of m1-2: (0) triangular; (1) heart-shaped; (2) laminar; (3) oval.

421. Shape of the posterior lobe of m1-2: (0) triangular; (1) heart-shaped; (2) laminar; (3) oval; (4) complex heartshaped.

422. Complete fusion of cristids on little worn lower molars: (0) absent; (1) present.
423. Complete fusion of cristids on moderately worn lower molars: (0) absent; (1) present.

424. Fusion of the med. I and the second cristid in an anterior lobe with wear on 1 1-2 (at an advanced degree of wear): (0) absent; (1) present.

425. Fusion of the med. I, the second cristid and the hypolophid (a part or entire) in an anterior lobe with wear on $m 1-2$ (at an advanced degree of wear): (0) absent; (1) present.

426. Lamines on lower molars: (0) absent; (1) present.

427. Number of lamines on lower molars (at an advanced degree of wear): (0) three; (1) four.

428. Secondary internal flexid (h.s.i.) on m1-2: (0) absent; (1) present.

429. Tertiary internal flexid (h.t.i.) on m1-2: (0) absent; (1) present.

430. Primary internal flexid (h.p.i.) on m1-2: (0) absent; (1) present.

431. Fundamental external flexid (h.f.e.) on m1-2: (0) absent; (1) present.

432. Secondary external flexid (h.s.e.) on m1-2: (0) absent; (1) present.

\section{CRANIO-MANDIBULAR CHARACTERS}

433. *Mandible: (0) sciurognathous; (1) hystricognathous; (2) pseudo-sciurognathous.

It is assumed that taxa from the same genus present the same condition regarding the mandibule structure.

434. * Length of the lower diastema with respect of the length of the toothrow: (0) very short (equal or shorter than the premolar-m1 distance); (1) short (between the premolar-m1 and premolar-m3 distances); (2) long (equal than the premolar-m3 distance).

435. Mandible at the level of the lower diastema: (0) low; (1) high.

436. Mental foramen: (0) present; (1) absent.

437. * Location of the mental foramen on the anterior region of the body of the mandible: (0) at the dorsal midpoint or below of the body of the mandible height; (1) above the dorsal midpoint of the body of the mandible height; (2) very close to the dorsal margin of the body of the mandible.

438. Position of the mandibular foramen: (0) behind the retromolar fossa; (1) at the level or close to the retromolar fossa (including below); (2) below the $\mathrm{m} 3$.

439. Notch for the insertion of the tendon of the m. masseter medialis pars infraorbitalis with respect to the toothrow: (0) isolated, located between the masseteric crest and the horizontal crest; (1) connected to the masseter crest; (2) connected to the horizontal crest; (3) connected to the lateral crest; (4) double connection: to the masseteric crest and the lateral crest. 
440. * Location of the notch for the insertion of the tendon of the $m$. masseter medialis pars infraorbitalis with respect to the toothrow: (0) below $\mathrm{p} 4$ or between $\mathrm{p} 4$ and $\mathrm{m} 1$; (1) below $\mathrm{m} 1$; (2) between $\mathrm{m} 1$ and $\mathrm{m} 2$; (3) below $\mathrm{m} 2$ or posterior to $\mathrm{m} 2$.

441. *Development of the masseter crest: (0) well-developed; (1) moderately developed; (2) absent or poorly developed as a thin and low ridge.

442. *Anterior origin of the masseteric crest with respect to the toothrow: $(0)$ below $\mathrm{m} 1$; (1) below $\mathrm{m} 2$; (2) below $\mathrm{m} 3$ or posterior to the $\mathrm{m} 3$.

443. *Development (thickness) of the anterior part of the lateral crest: (0) well-developed; (1) moderately developed; (2) absent or poorly developed.

444. * Development of the posterior part of the horizontal crest: (0) absent or extremely reduced; (1) present as a low and broad ridge; (2) present as a conspicuous crest, forming a laterally projected shelf but lacking a dorsal fossa (or a shallow fossa); (3) well-developed, forming a laterally projected shelf and bearing a deep fossa on its dorsal surface.

445. Development of the anterior part (below p4-m2) of the horizontal crest: (0) absent or extremely reduced; (1) well-developed, forming a laterally projected shelf and bearing a fossa on its dorsal surface.

446. * Depth of the fossa located dorsally to the horizontal crest (fossa for the insertion of the zygomaticomandibular): (0) shallow; (1) moderatly deep; (2) deep.

447. Coronoid process: (0) well-defined; (1) not distinguishable.

448. Height of the coronoid process compared to the position of the condyle: (0) located at the same dorsoventral level than the condyle or higher; (1) located more ventrally than the condyle.

449. Condyloid apophysis: (0) high; (1) low.

450. Length of the post-condylar process: (0) shorter than the anteroposterior lenght of the condyle; (1) equal or longer than the anteroposterior lenght of the condyle.

451. * Posterior extension of the angular process: $(0)$ at the level of the post-condylar process; (1) ending posteriorly to the post-condylar process.

452. *Dorsoventral development of the angular process with respect to the ascendant ramus $(H)$ : $(0)$ weak $(\mathrm{H}<0.3)$; (1) moderate $(0.3<\mathrm{H}<0.4)$; (2) strong $(\mathrm{H}>0.4)$.

453. Alveolar protuberances: (0) absent; (1) present.

454. Anterior projection of the premaxillary bone (in ventral view): (0) absent; (1) present.

455. * Development of the anterior projection of the premaxillary bone (in ventral view): (0) poorly developed; (1) well-developed; (2) strongly developed.

456. Length of the rostrum with respect to the skull length $(L)$ : (0) short $(\mathrm{L}<0.25)$; (1) long $(\mathrm{L}>0.25)$.

457. ${ }^{*}$ Length of the incisive foramina with respect of length of the toothraw: (0) long (equal to the premolar-M2 distance);
(1) medium (inferior to the premolar-M2 distance and superior or equal to the premolar-M1 distance); (2) short (inferior to the premolar-M1 distance).

458. * Shape of the incisive foramina: (0) anterior width superior to the posterior one; (1) anterior and posterior widths equal or roughly equal; (2) posterior width superior to the one anterior.

459. * Palatal ridges formed by the maxillary, posteriorly to the incisive foramen: (0) absent or poorly developed; (1) well-developed; (2) strongly developed.

460. Margins of the maxillary posteriorly to the incisive foramina: (0) separated; (1) not separated and forming a crest; (2) not separated and not forming a crest, but roughly plate surface.

461. * Length of the upper diastema with respect to the length of the toothrow: (0) longer than the premolar-M3 distance; (1) equal than the premolar-M3 distance; (2) shorter than the premolar-M3 distance.

462. * Posterior extremity of the fusion of both maxillaries in ventral view: (0) at the level of the premolar; (1) at the level of the M1; (2) at the level of the M2-3.

463. Articulation of the nasals with respect to the premaxillary: (0) nasals articulate with the premaxillary almost throughtout their lenght; (1) anterior part of the nasals does not articulate with the premaxillary.

464. Interorbital width: (0) irregular/with constriction (minimum width/maximum width $<0.8)$; (1) regular/without constriction (minimum width/maximum width $>=$ $0.8)$.

465. Posterior suture of frontals with parietals: (0) neither convex nor concave nor perpendicular to the suture between both frontals; (1) roughly perpendicular to the suture between both frontals or concave; (2) convex.

466. Development of the infraorbital foramen: (0) small; (1) large.

467. Maxillary on the anterior branch of the orbit (ascendant ramus): (0) continuous; (1) discontinuous.

468. Contact between the jugal and the lacrimal on the anterior branch of the orbits (anteorbital zygomatic bar; ascendant ramus): (0) absent; (1) present.

469. * Development of the lacrimal on the anterior branch of the orbit: (0) large; (1) medium; (2) small.

470. Development of the lacrimal: (0) small (contact between the maxilla and the frontal); (1) strong (no contact between the maxilla and the frontal).

471. Postorbital process (zygomatic process of the frontal): (0) absent; (1) present.

472. Development of the postorbital process: (0) small; (1) large.

473. Conformation of the postorbital process: (0) only frontal; (1) frontal and squamosal; (2) only squamosal.

474. Frontal process of the zygomatic (superior jugal process of Emmons 2005; paraorbital process of Verzi et al. 2016): (0) absent; (1) present. 
475. Position of the frontal process of the zygomatic: (0) on only jugal; (1) at the limit of the jugal and squamosal.

476. Inferior jugal process (Emmons 2005): (0) absent; (1) present.

477. * Position of the inferior jugal process with respect of the frontal process of the zygomatic: (0) anterior; (1) same level; (2) posterior.

478. Depth of the masseteric fossa of the zygomatic arch (suborbital fossa; jugal fossa): (0) shallow or absent; (1) deep.

479. Length of the masseteric fossa of the zygomatic arch: (0) short; (1) long (reaches the anteroventral fossa of the zygomatic arch).

480. Development of the zygomatic arch: (0) moderate; (1) strong.

481. *Development of the foramen into the lacrimal canal: (0) absent or small; (1) moderate; (2) well-developed.

482. Association between the foramen into the lacrimal canal and the lacrimallacrimal foramen: (0) absent; (1) present.

483. Position of the lacrimal foramen (nasolacrimal foramen): (0) opens into the orbital portion of the lacrimal; (1) opens into the maxilla.

484. Exposure of the lacrimal foramen: (0) not laterally exposed; (1) laterally exposed.

485. *Position of the ethmoidal foramen: $(0)$ at the level of M1-2; (1) at the level of M2; (2) at the level of M2-3; (3) at the level of M3.

486. *Development of the sphenopalatine foramen/fissure: (0) well-developed; (1) moderate; (2) small.

487. Position of the sphenopalatine foramen with respect to the lacrimal foramen: (0) ventral; (1) posteroventral.

488. Palatine foramen (for the palatine artery and palatine nerve): (0) well-developed; (1) small or absent.

489. Sphenopalatine (pterygopalatine) vacuities: (0) absent; (1) present.

490. Development of the pterygoids: (0) long (reaches the auditory bullae); (1) short.

491. *Apex of the mesopterygoid fossa (choanae, internal nares) with respect of M2: (0) apex in front of M2; (1) at the level of M2-3; (2) at the level of the posterior end of the toothraw or behind the toothrow.

492. Anterior margins of the mesopterygoid fossa (choanae, internal nares): (0) triangular/convex; (1) subperpendicular of the anteroposterior axis of the skull; (2) nor triangular/convex neither subperpendicular of the anteroposterior axis of the skull.
493. Lateral development of the mesopterygoid fossa (choanae, internal nares): (0) strong; (1) weak.

494. Development of the oval foramen: (0) small; (1) welldeveloped.

495. Masticory and/or buccinator foramina: (0) present; (1) absent.

496. Fusion of the masticory foramen with the buccinator fordmen: (0) absent; (1) present.

497. Fusion of the jugular foramen with the carotid foramen: (0) absent; (1) present.

498. Contact between the maxillary and the alisphenoid in the basisphenoid region: (0) present; (1) absent.

499. Development of the temporal fossae: (0) shallow; (1) deep.

500. Development of the interparietal on the skull roof: (0) welldeveloped (parietals well-separated from the occiput); (1) small (parietal close to the occiput).

501. Sagittal crest: (0) absent; (1) present (always or sometimes).

502. Orientation of the paroccipital process (on the supraoccipital): (0) ventral or ventrolaterally oriented; (1) laterally oriented.

503. Paroccipital process and bullae: (0) separated or partially joined; (1) completely joined.

504. Length of the paroccipital process: (0) short; (1) long.

505. Development of the paroccipital process: (0) small; (1) large.

506. Mastoid process: (0) absent; (1) present.

507. Lateral process formed by the supraoccipital (and interparieta, which can be also associated with the mastoid process): (0) absent; (1) present.

508. Development of the lateral process: (0) weak; (1) strong.

509. Epitympanic sinus (postglenoid fossa): (0) partially or not covered by the posterior apophysis of the squamosal; (1) completly covered by the posterior apophysis of the squamosal.

510. Thickness of the ventral margin of the posterior process of the squamosal: (0) thin; (1) thick.

511. Development of the external auditory meatus: (0) small; (1) large.

512. Accessory foramen below the external auditory meatus: (0) present; (1) absent.

513. Development of the accessory foramen below the external auditory meatus: (0) small; (1) large. 
ANNEXE 2. - Matrice taxons-caractères utilisée pour les analyses cladistiques réalisées lors du présent travail.

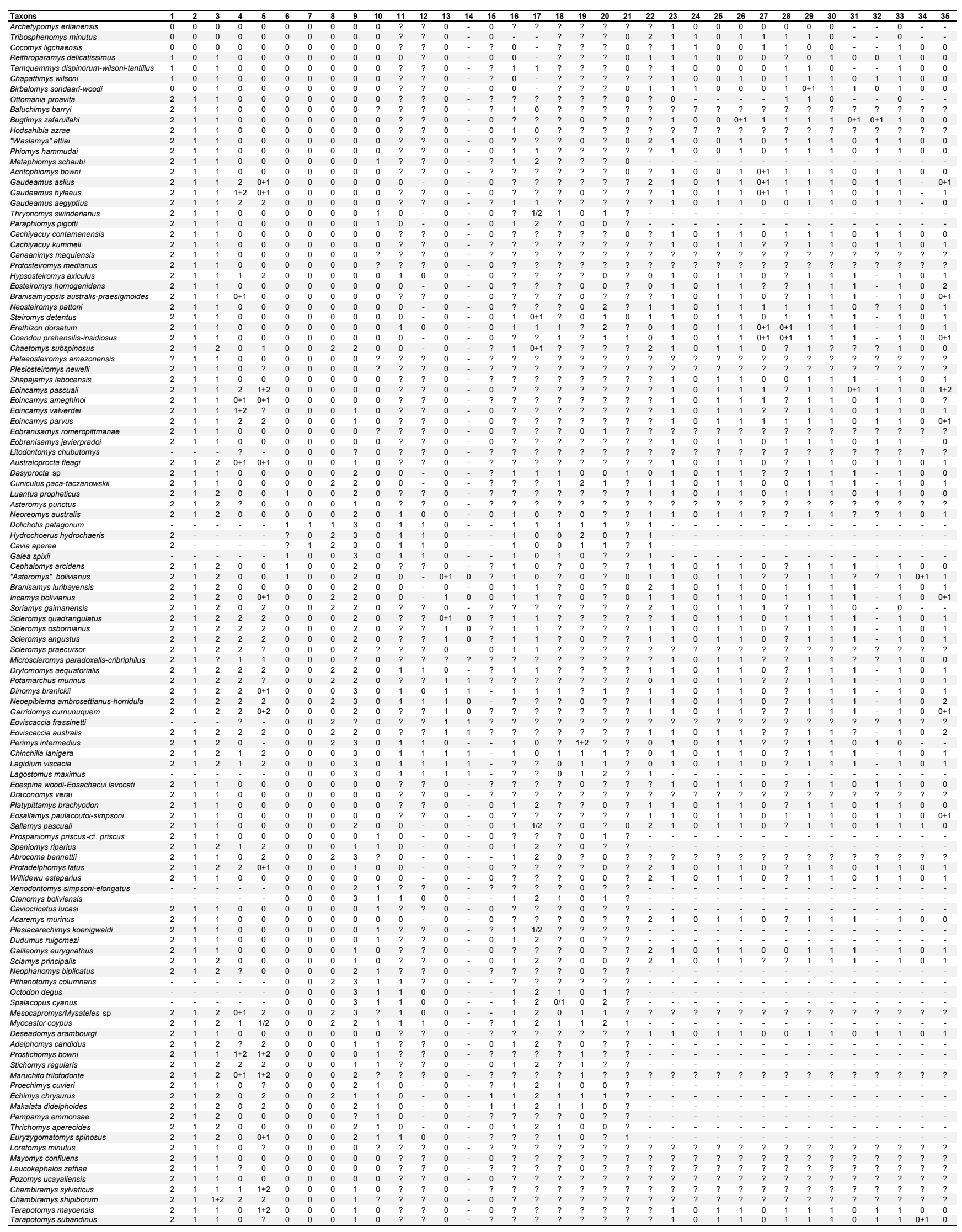




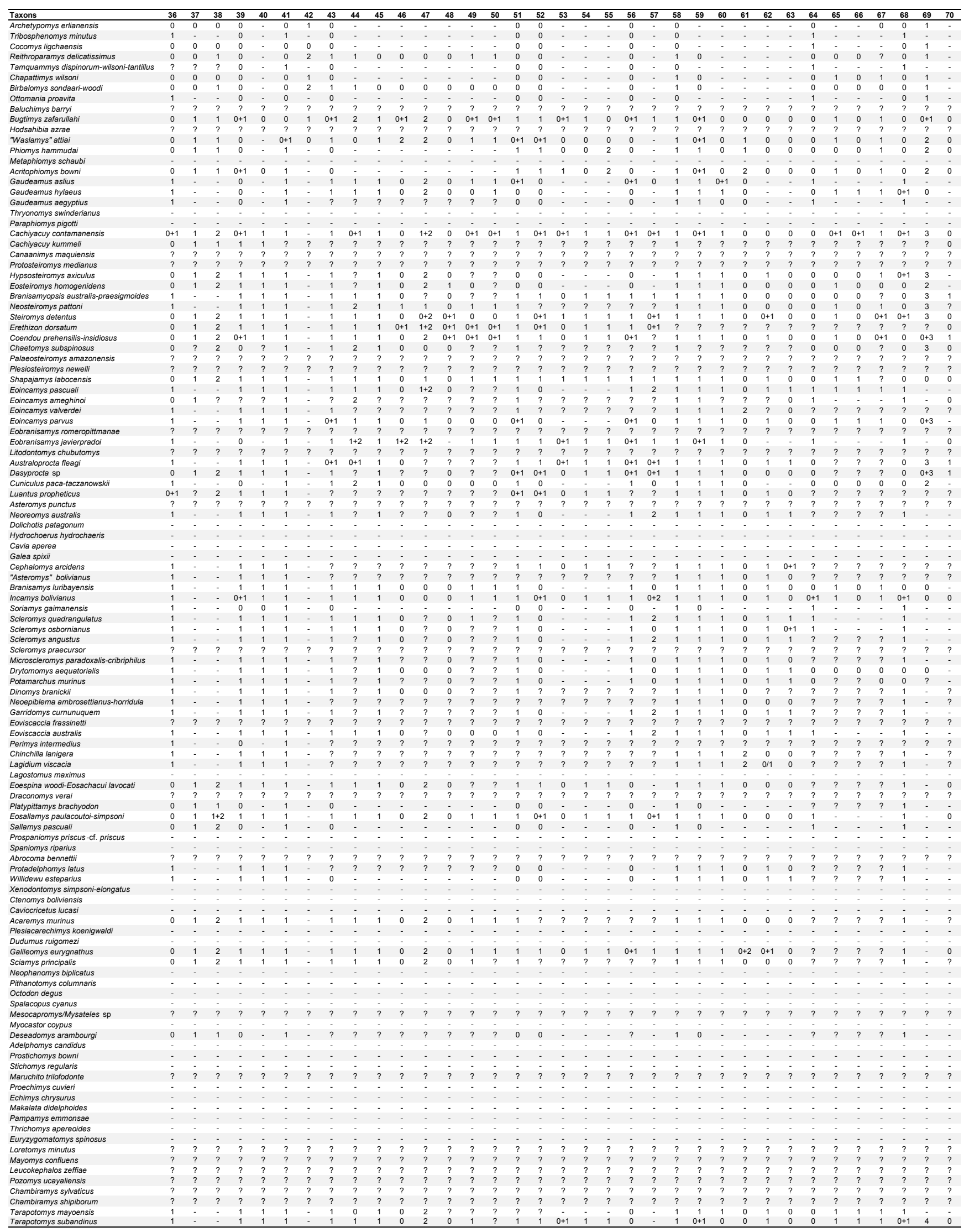




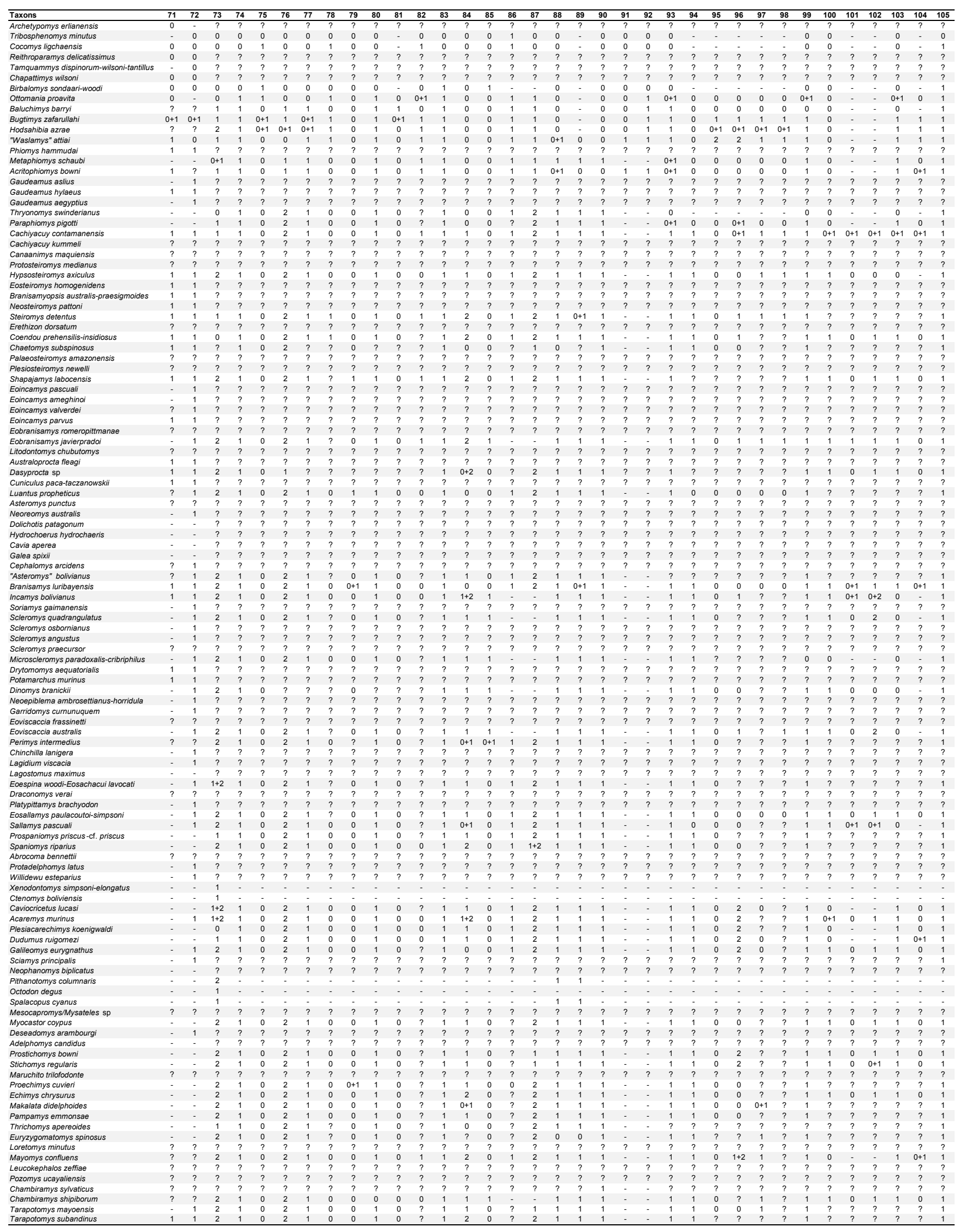




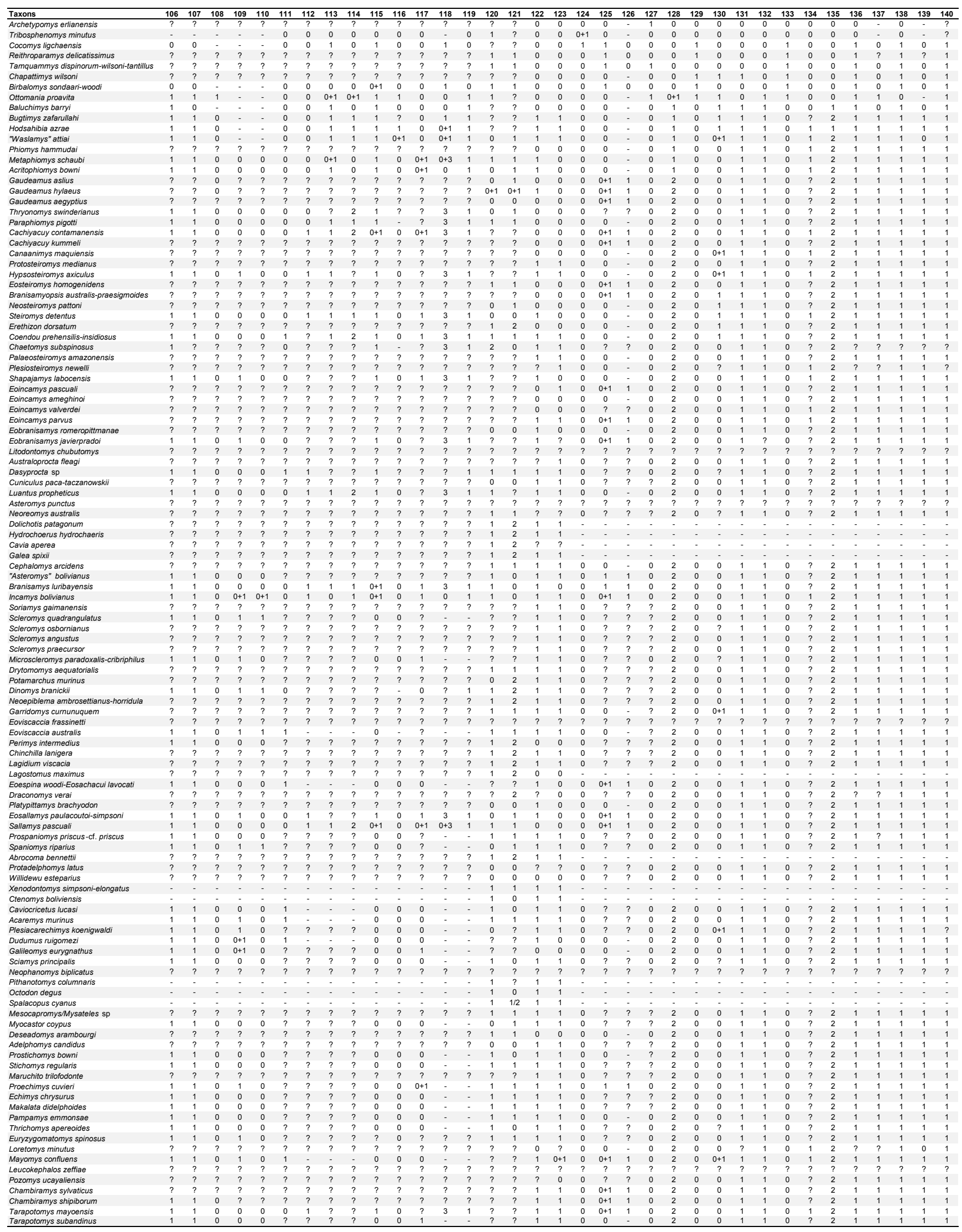




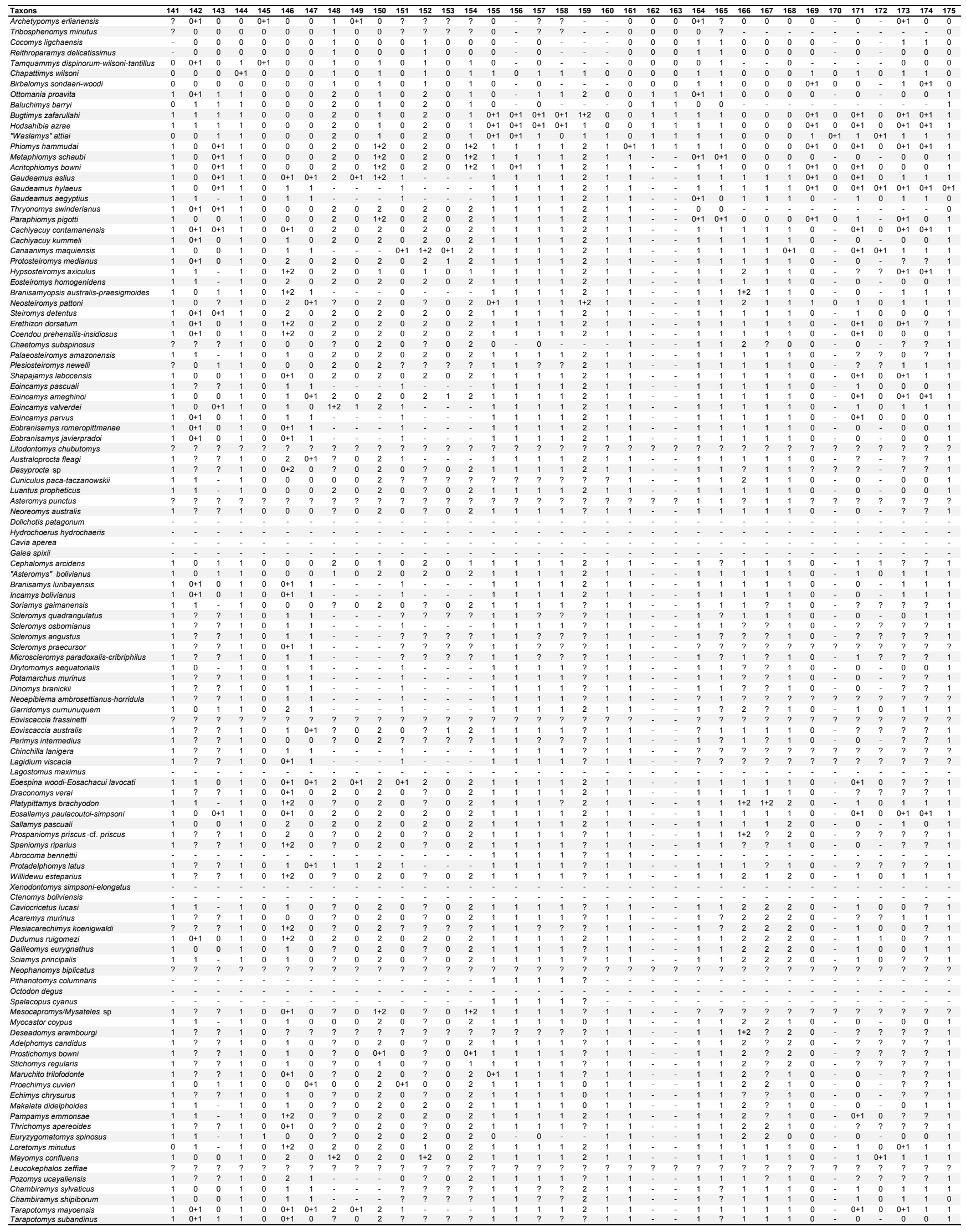




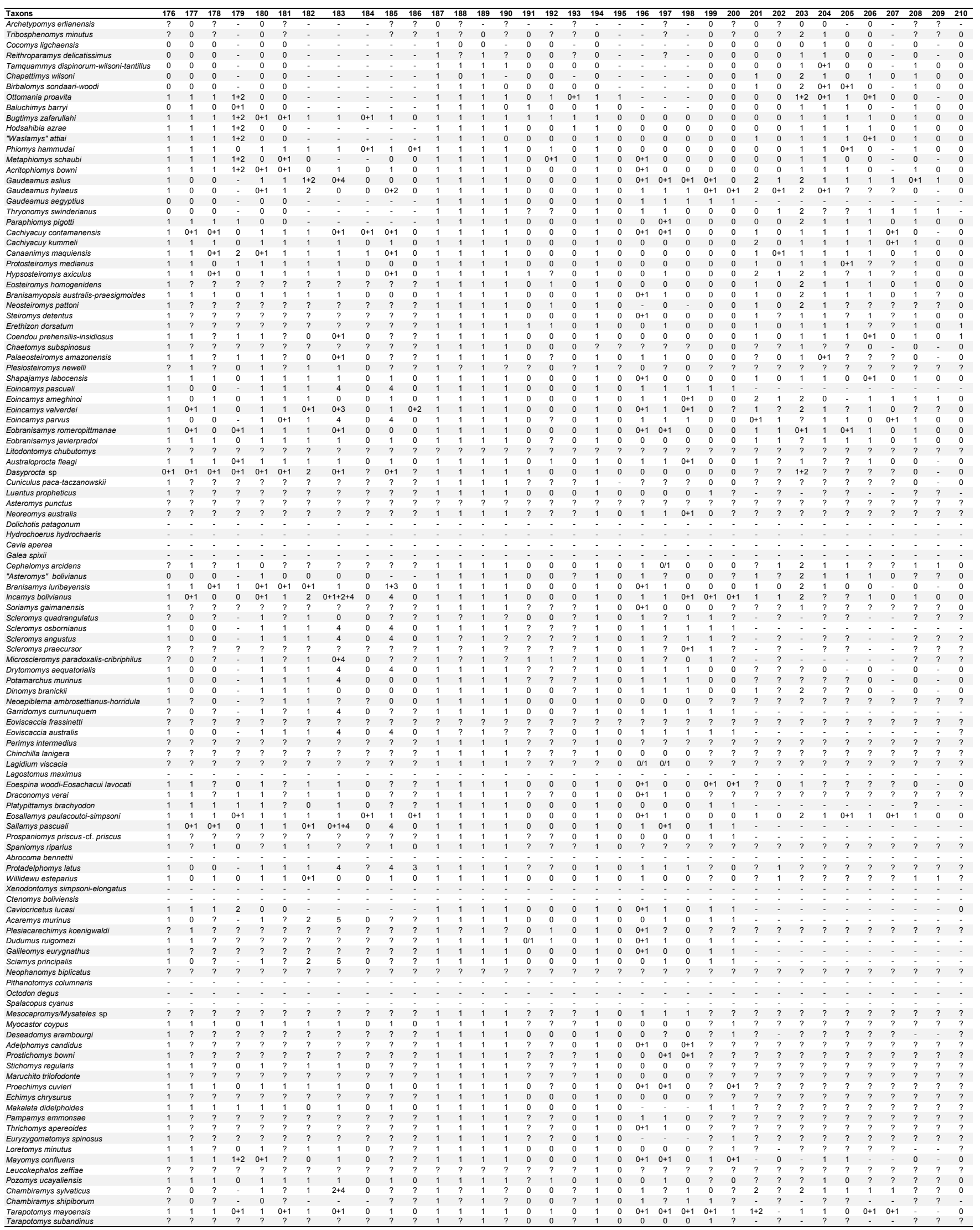




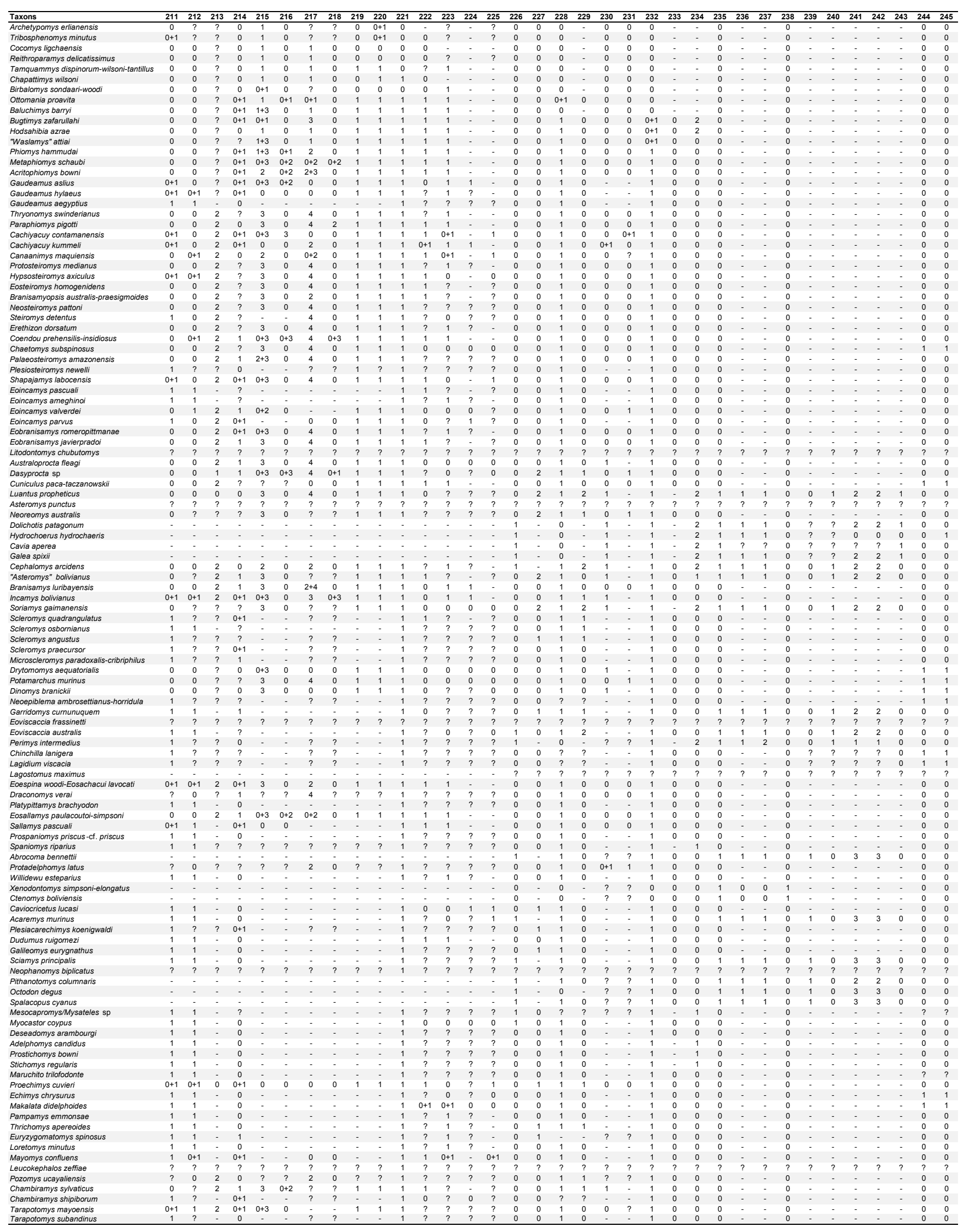




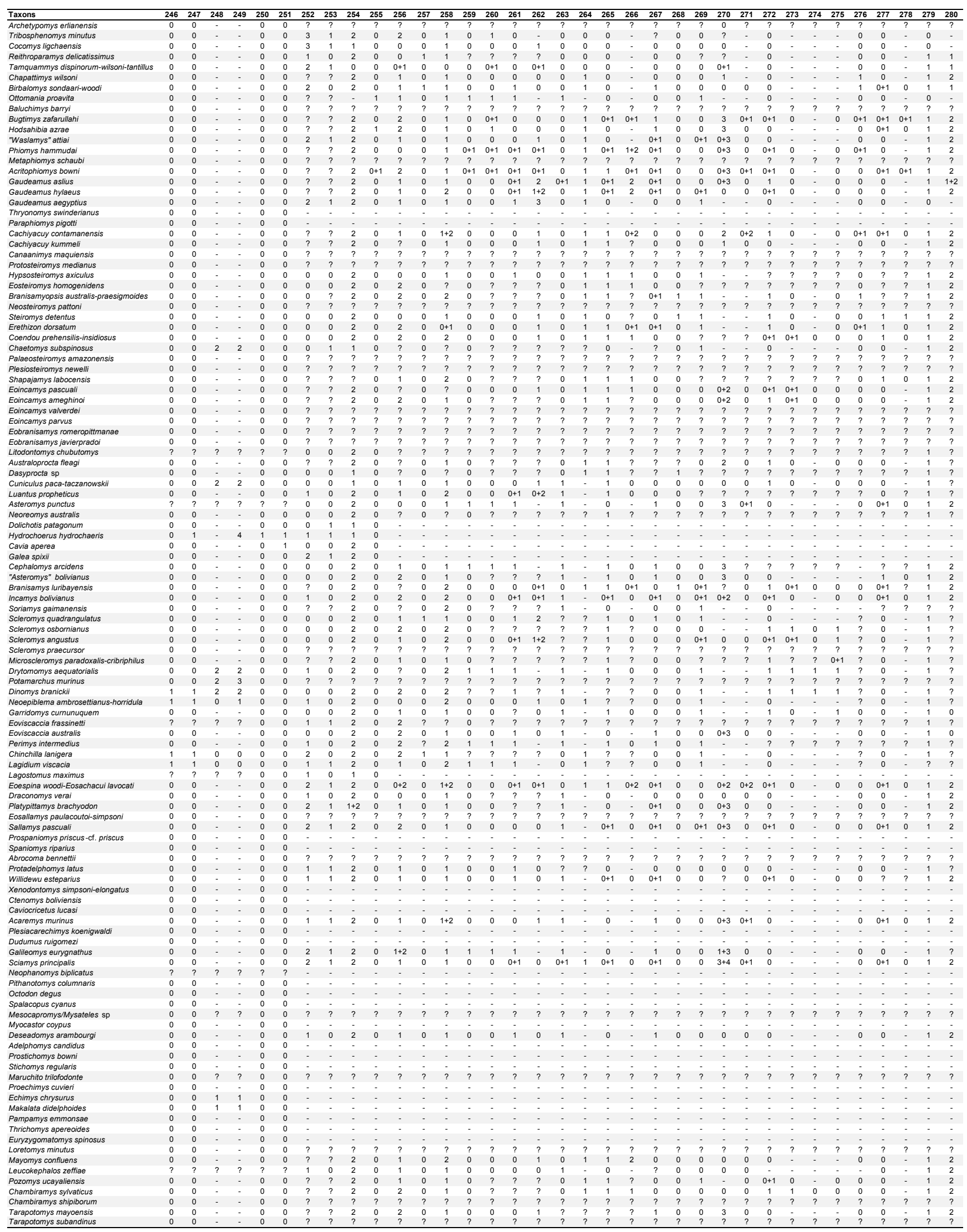




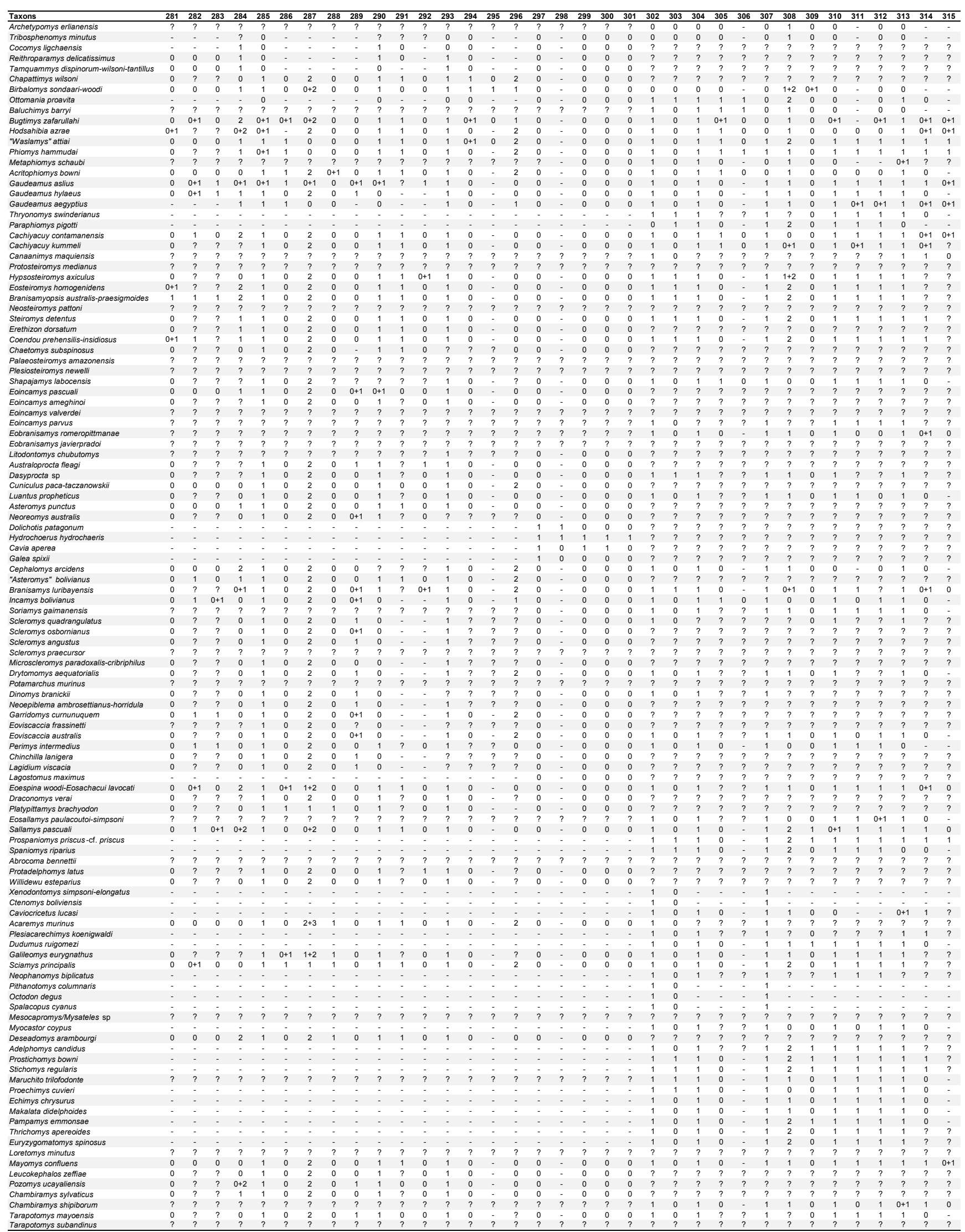




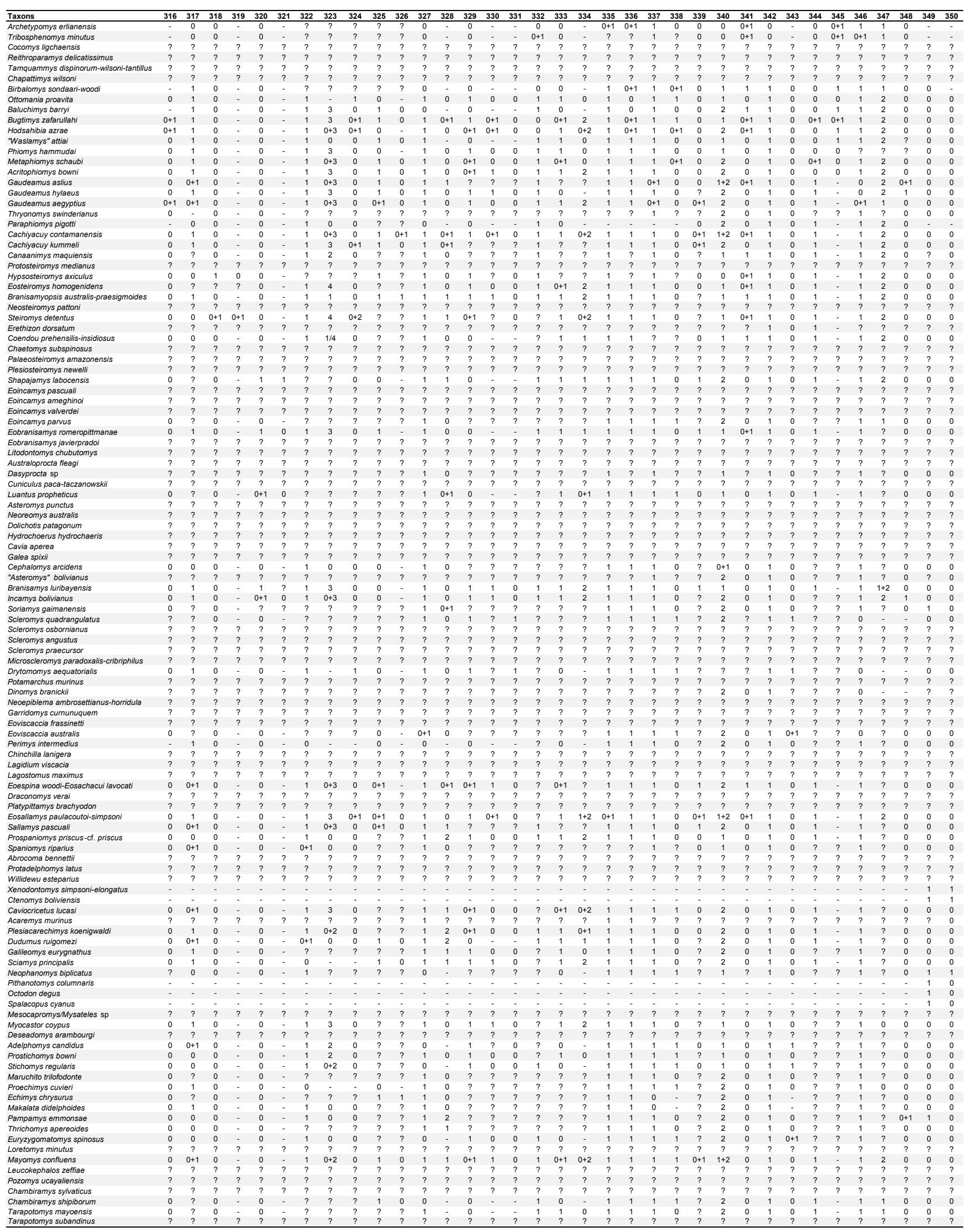




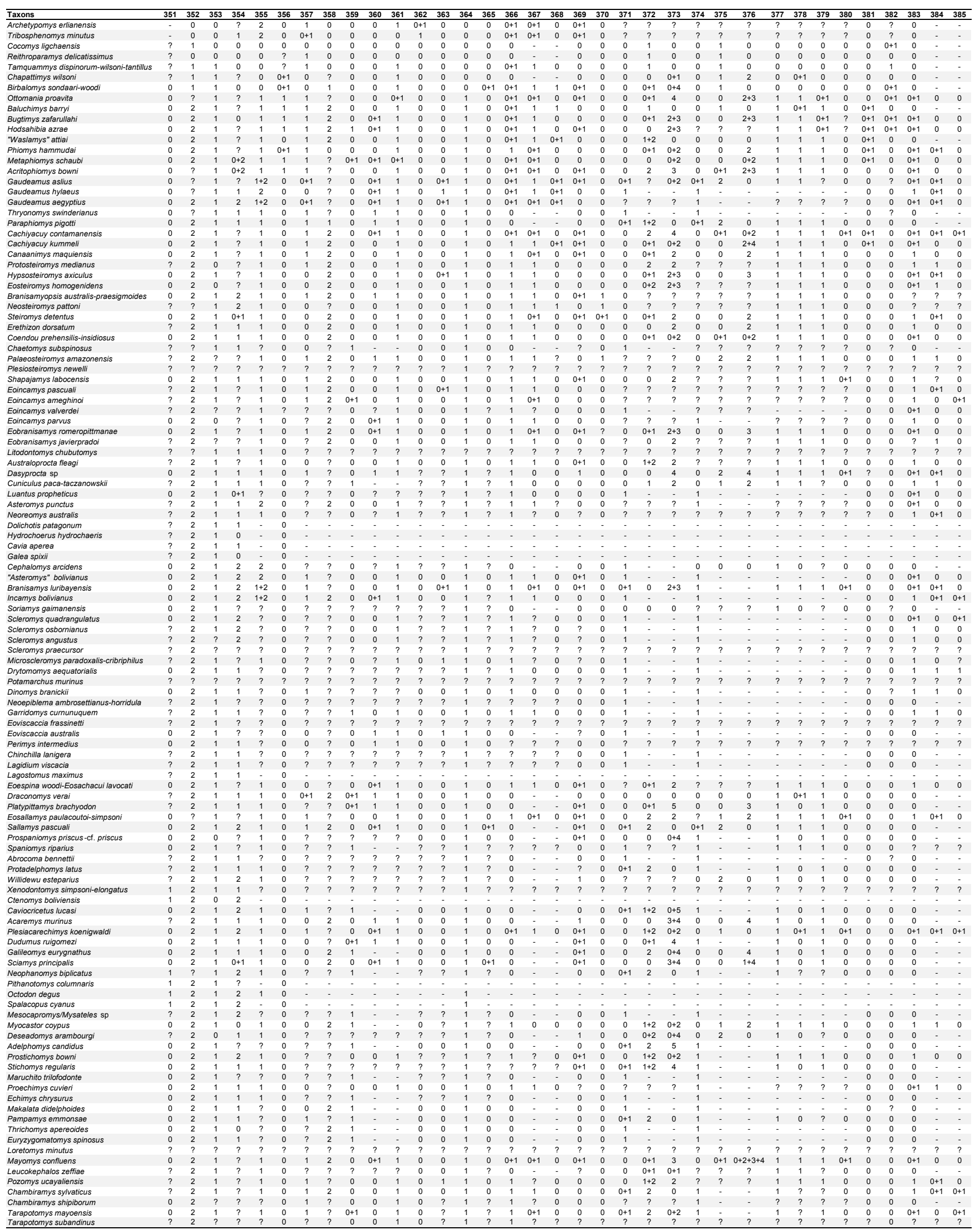




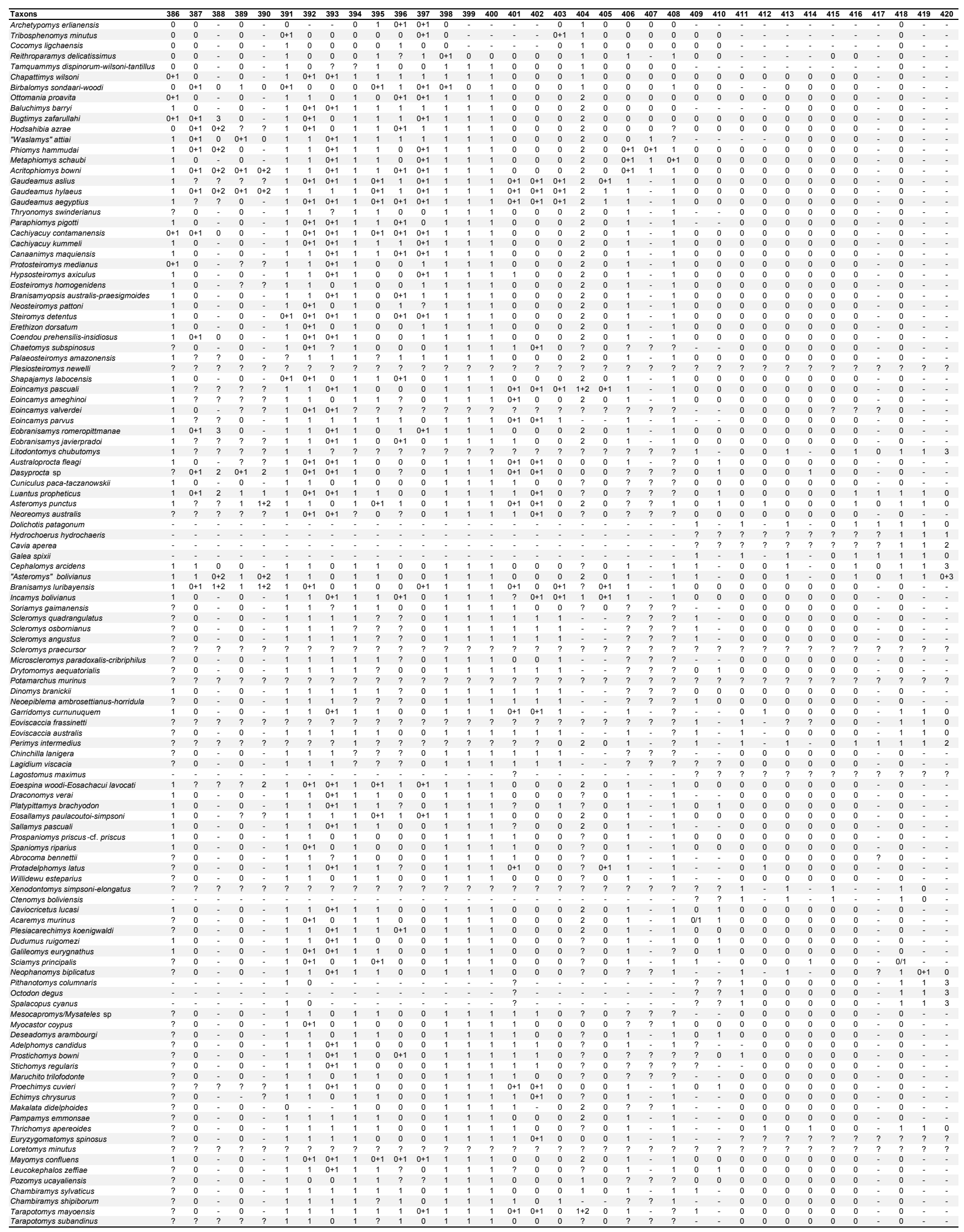




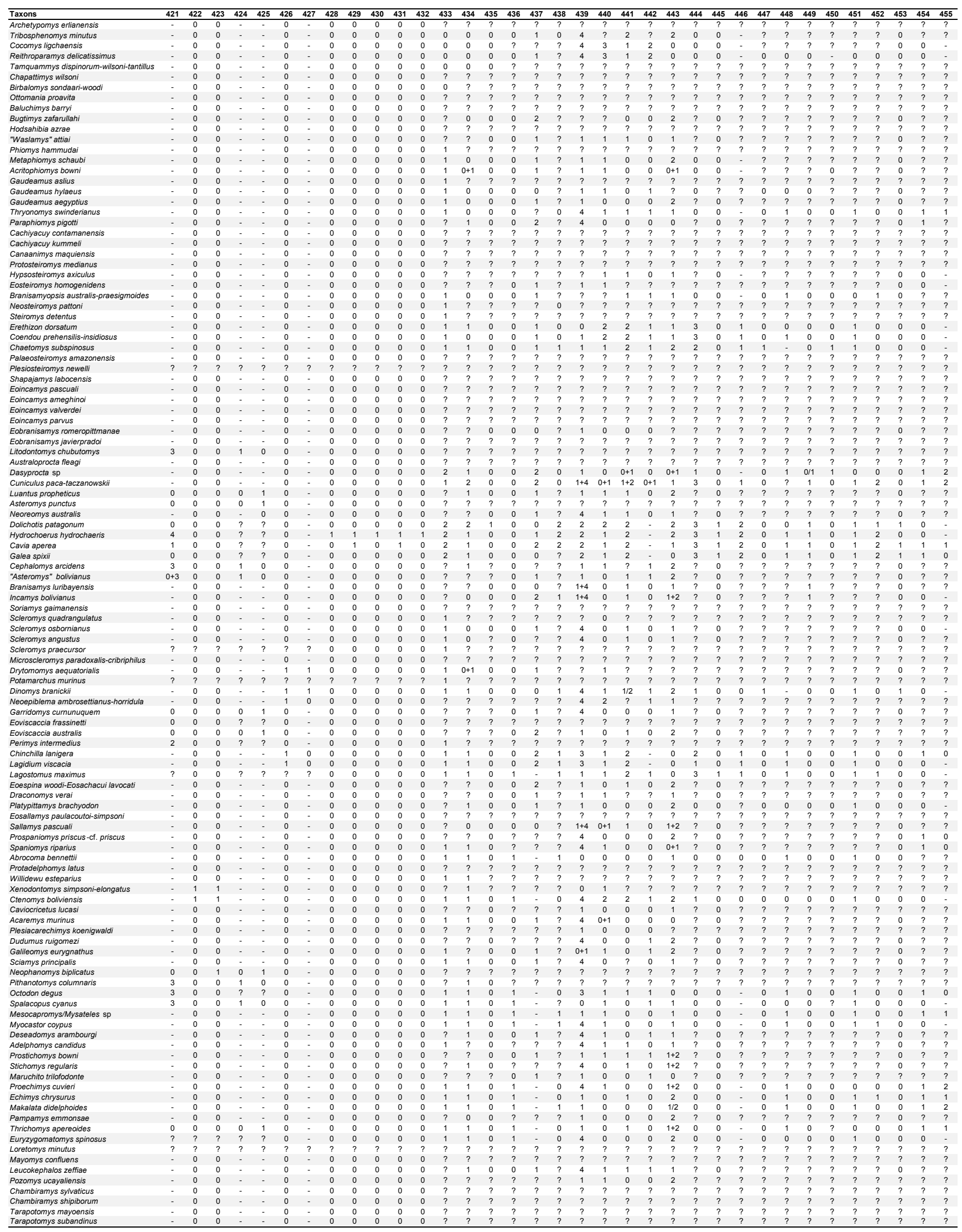




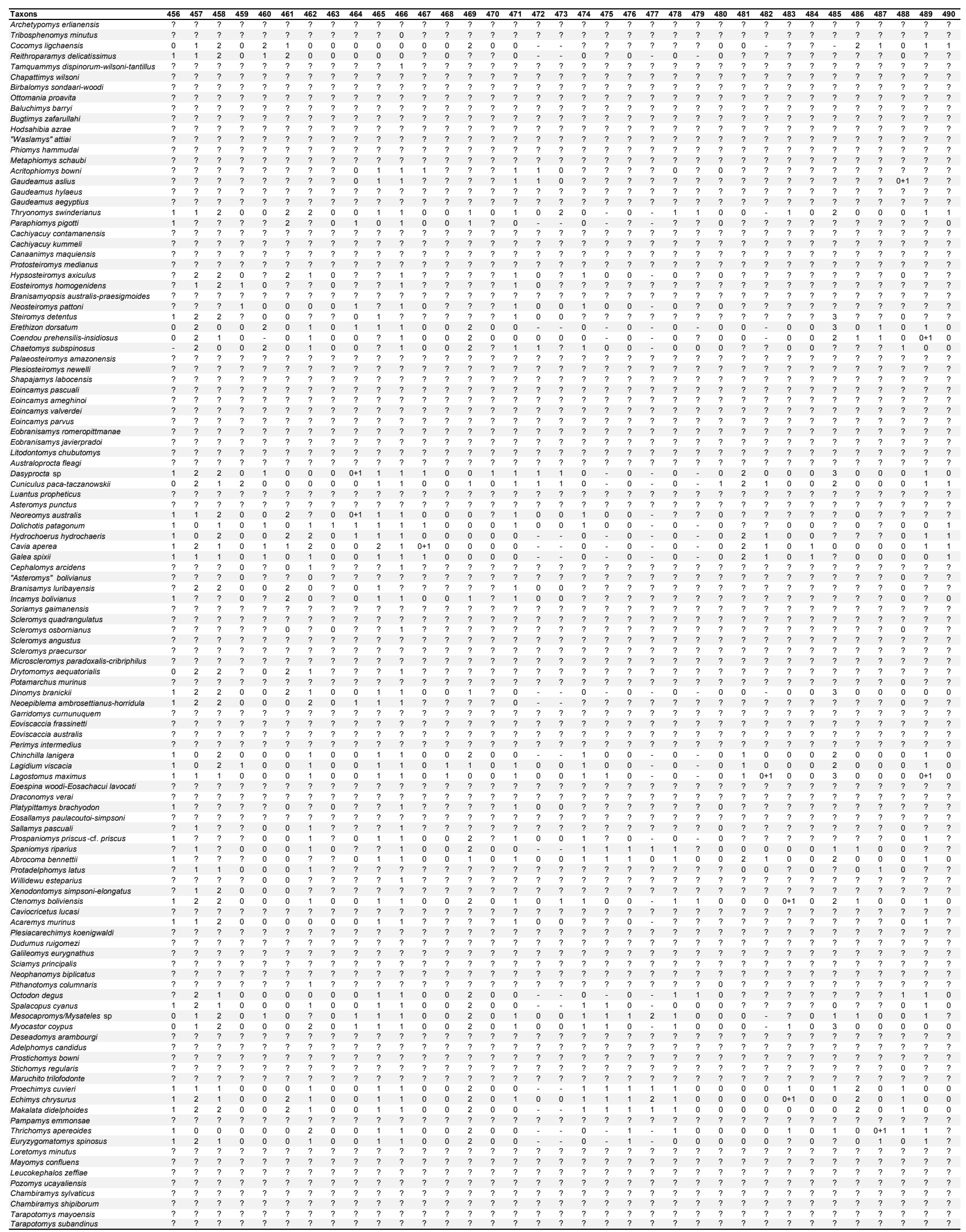


ANNEXE 2. - Suite.

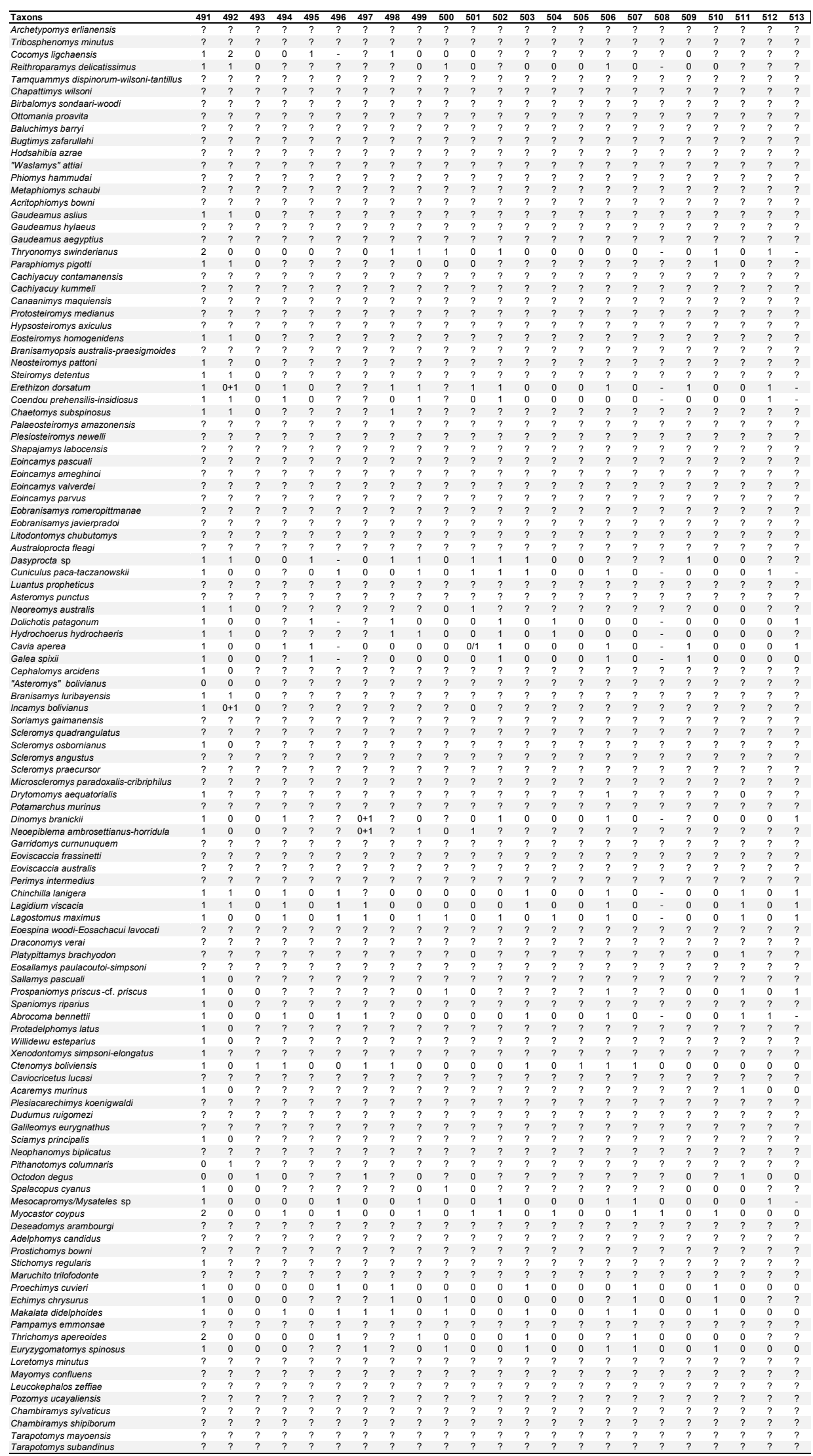


ANNEXE 3. - Liste des taxons inclus dans les analyses cladistique réalisées lors du présent travail. Abréviations: Fm, Formation; INGEMMET, Instituto Geológico Minero y Metalúrgico, Lima; MACN, Museo Argentino de Ciencias Naturales, Buenos Aires; MLP, Museo de Ciencias Naturales de La Plata; MUSM, Museo de Historia Natural de la Universidad Nacional Mayor San Marcos, Lima; MNHN-Bol, Museo Nacional de Historia Natural, La Paz, Bolivia; MNHN, Muséum national d'Histoire naturelle, Paris; UFAC, Laboratorio de Pesquisa Palontolegica, Universidad Federal do Acre; UM, Université de Montpellier. Références utilisées: Ameghino 1887, 1894, 1897, 1902, 1903; Anthony 1922; Antoine et al. 2012; Arnal \& Kramarz 2011; Arnal \& Pérez 2013; Arnal \& Vucetich 2011; Arnal \& Vucetich 2015b; Arnal et al. 2014; Bertrand et al. 2012; Boivin et al. 2017a, b, 2018a; De Bruijn et al. 2003; Busker \& Dozo 2017; Candela 2000, 2003, 2004; Candela \& Nasif 2006; Candela \& Vucetich 2002; Candela et al. 2012. Dawson et al. 1984; Fields 1957; Flynn et al. 1986, 2002; Frailey \& Campbell 2004; Hoffstetter \& Lavocat 1970; Hussein et al. 1978; Jaeger et al. 2010; Kay et al. 2012; Kerber et al. 2017; Kramarz 1998, 2001a, b, c, 2004, 2006b; Kramarz et al. 2010, 2013; Lavocat 1973, 1976; Leidy 1871; Li et al. 1989; Loomis 1914; Martin 1994; Marivaux \& Welcomme 2003; Marivaux et al. 2002; Meng \& Wyss 2001; Meng et al. 2007a, b; Olivares et al. 2012; Patterson \& Wood 1982; Pérez \& Vucetich 2012b; Pérez et al. 2018; Sallam et al. 2009, 2011, 2012; Verzi 1999; Verzi et al. 1991, 1995, 1999, 2003, 2011; Vucetich \& Bond 1984 ; Vucetich \& Verzi 1991, 1994, 1996; Vucetich \& Vieytes 2006; Vucetich et al. 1992, 1993a, 2010a, b, 2015b; Walton 1990, 1997; Wood 1949, 1962, 1968; Wood \& Patterson 1959.

\begin{tabular}{|c|c|c|c|c|c|}
\hline Genus & Species & $\begin{array}{l}\text { Locality and/or } \\
\text { Formation }\end{array}$ & Age & $\begin{array}{r}\text { Observed material } \\
\text { (original or cast) }\end{array}$ & Primary references \\
\hline Archetypomys & A. erlianensis & $\begin{array}{l}\text { Nuhetingboerhe, Lower } \\
\text { Arshanto Formation, } \\
\text { Inner Mongolia }\end{array}$ & late early Eocene & & Meng et al. 2007a \\
\hline Tribosphenomys & T. minutus & Subeng, Inner Mongolia & $\begin{array}{l}\text { late Palaeocene } \\
\text { - earliest } \\
\text { Eocene }\end{array}$ & & $\begin{array}{r}\text { Meng \& Wyss 2001; } \\
\text { Meng et al. 2007b }\end{array}$ \\
\hline Cocomys & C. lingchaensis & Hengdong County, China & early Eocene & UM (cast) & Li et al. 1989 \\
\hline Reithroparamys & R. delicatissimus & $\begin{array}{l}\text { Wasatch Formation, } \\
\text { Wyoming, USA }\end{array}$ & early Eocene & UM (cast) & $\begin{array}{l}\text { Leidy 1871; Wood } \\
\quad 1962\end{array}$ \\
\hline Tamquammys & $\begin{array}{l}\text { T. dispinorum-wilsoni- } \\
\text { tantillus }\end{array}$ & $\begin{array}{l}\text { Andarak 2, Kyrgyzstan; } \\
\text { Khaychin, Arshanto, } \\
\text { Mongolia }\end{array}$ & $\begin{array}{l}\text { late early - early } \\
\text { middle Eocene }\end{array}$ & & Dawson et al. 1984 \\
\hline Chapattimys & C. wilsoni & $\begin{array}{l}\text { H-GSP 144, Kala Chitta } \\
\text { Range (Kuldana Fm), } \\
\text { Pakistan }\end{array}$ & middle Eocene & & Hussain et al. 1978 \\
\hline Birbalomys & B. sondaari-woodi & $\begin{array}{l}\text { H-GSP 144, Kala Chitta } \\
\text { Range (Kuldana Fm), } \\
\text { Pakistan }\end{array}$ & middle Eocene & UM (cast) & Hussain et al. 1978 \\
\hline Ottomania & O. proavita & $\begin{array}{l}\text { Süngülü, Lesser Caucasus, } \\
\text { Turkey }\end{array}$ & $\begin{array}{l}\text { Eocene/ } \\
\text { Oligocene } \\
\text { boundary } \\
\text { interval }\end{array}$ & & De Bruijn et al. 2003 \\
\hline Baluchimys & B. barryi & $\begin{array}{l}\text { Y-GSP 417, Baluchistan, } \\
\text { Pakistan }\end{array}$ & early Oligocene & & Flynn et al. 1986 \\
\hline Bugtimys & B. zafarullahi & $\begin{array}{l}\text { Paali DBC2, Bugti Hills, } \\
\text { Pakistan }\end{array}$ & early Oligocene & UM (original) & Marivaux et al. 2002 \\
\hline Hodsahibia & H. azrae & $\begin{array}{l}\text { Paali DBC2, Bugti Hills, } \\
\text { Pakistan }\end{array}$ & early Oligocene & UM (original) & $\begin{array}{l}\text { Flynn et al. 1986; } \\
\text { Marivaux \& } \\
\text { Welcomme } 2003\end{array}$ \\
\hline «Waslamys» & «W». attiai & $\begin{array}{l}\text { BQ-2, Birket Qarun Loc. 2, } \\
\text { Fayum, Egypt }\end{array}$ & early late Eocene & UM (cast) & Sallam et al. 2009 \\
\hline Acritophiomys & A. bowni & $\begin{array}{l}\text { L-41 (Jebel el Qatrani Fm), } \\
\text { Fayum, Egypt }\end{array}$ & latest Eocene & & Sallam et al. 2012 \\
\hline Phiomys & P. hammudai & DT-1\&2, Dur At-Talah, Libya & $\begin{array}{l}\text { late ?middle - } \\
\text { late Eocene }\end{array}$ & UM (cast) & Jaeger et al. 2010 \\
\hline Metaphiomys & M. schaubi & $\begin{array}{l}\text { Y Quarry B, E, G (Jebel } \\
\text { el Qatrani Fm), Fayum, } \\
\text { Egypt; Zallah, Libya }\end{array}$ & early Oligocene & UM (cast) & Wood 1968 \\
\hline \multirow[t]{3}{*}{ Gaudeamus } & G. aegyptius & $\begin{array}{l}\text { Jebel el Qatrani Fm, } \\
\text { Fayum, Egypt }\end{array}$ & lastest Eocene & & Wood 1968 \\
\hline & G. aslius & $\begin{array}{l}\text { L-41 (Jebel el Qatrani Fm), } \\
\text { Fayum, Egypt }\end{array}$ & lastest Eocene & & Sallam et al. 2011 \\
\hline & G. hylaeus & $\begin{array}{l}\text { L-41 (Jebel el Qatrani Fm), } \\
\text { Fayum, Egypt }\end{array}$ & lastest Eocene & & Sallam et al. 2011 \\
\hline Paraphiomys & P. pigotti & Rusinga, Kenya & early Miocene & UM (original) & Lavocat 1973 \\
\hline Thryonomys & T. swinderianus & & Recent & UM (original) & \\
\hline \multicolumn{6}{|l|}{ CAVIOMOPHA } \\
\hline \multirow[t]{2}{*}{ Cachiyacuy } & C. contamanensis & $\begin{array}{l}\text { Contamana CTA-27 (Pozo } \\
\text { Fm), Peru }\end{array}$ & $\begin{array}{l}\text { late middle } \\
\text { Eocene }\end{array}$ & MUSM (original) & $\begin{array}{l}\text { Antoine et al. 2012; } \\
\text { Boivin et al. 2017a }\end{array}$ \\
\hline & C. kummeli & $\begin{array}{l}\text { Contamana CTA-27 (Pozo } \\
\text { Fm), Peru }\end{array}$ & $\begin{array}{l}\text { late middle } \\
\text { Eocene }\end{array}$ & MUSM (original) & $\begin{array}{l}\text { Antoine et al. 2012; } \\
\text { Boivin et al. 2017a }\end{array}$ \\
\hline Canaanimys & C. maquiensis & $\begin{array}{l}\text { Contamana CTA-27 (Pozo } \\
\text { Fm), Peru }\end{array}$ & $\begin{array}{l}\text { late middle } \\
\text { Eocene }\end{array}$ & MUSM (original) & $\begin{array}{l}\text { Antoine et al. 2012; } \\
\text { Boivin et al. 2017a }\end{array}$ \\
\hline Pozomys & P. ucayaliensis & $\begin{array}{l}\text { Contamana CTA-29 (Pozo } \\
\text { Fm), Peru }\end{array}$ & $\begin{array}{l}\text { late middle } \\
\text { Eocene }\end{array}$ & MUSM (original) & Boivin et al. 2017a \\
\hline
\end{tabular}


ANNEXE 3. - Suite.

\begin{tabular}{|c|c|c|c|c|c|}
\hline Genus & Species & $\begin{array}{l}\text { Locality and/or } \\
\text { Formation }\end{array}$ & Age & $\begin{array}{r}\text { Observed material } \\
\text { (original or cast) }\end{array}$ & Primary references \\
\hline Leucokephalos & L. zeffiae & $\begin{array}{l}\text { Cabeza Blanca (Sarmiento } \\
\text { Fm), Argentina }\end{array}$ & $\begin{array}{l}\text { late early } \\
\text { Oligocene- } \\
\text { late Oligocene }\end{array}$ & UM (cast) & Vucetich et al. 2015b \\
\hline \multirow[t]{2}{*}{ Chambiramys } & C. shipiborum & $\begin{array}{l}\text { Contamana CTA-32 } \\
\text { (Chambira Fm), Peru }\end{array}$ & late Oligocene & MUSM (original) & Boivin et al. 2017b \\
\hline & C. sylvaticus & $\begin{array}{l}\text { Contamana CTA-61 } \\
\quad \text { (Chambira Fm), Peru }\end{array}$ & late Oligocene & MUSM (original) & Boivin et al. 2017b \\
\hline \multirow[t]{2}{*}{ Tarapotomys } & T. mayoensis & $\begin{array}{l}\text { TAR-01, Shapaja (Pozo } \\
\text { Fm), Peru }\end{array}$ & early Oligocene & MUSM (original) & Boivin et al. 2018a \\
\hline & T. subandinus & $\begin{array}{l}\text { TAR-21, TAR-22, Shapaja } \\
\text { (Pozo Fm), Peru }\end{array}$ & early Oligocene & MUSM (original) & Boivin et al. 2018a \\
\hline \multicolumn{6}{|l|}{ Octodontoidea } \\
\hline Eosallamys & $\begin{array}{l}\text { E. paulacoutoi- } \\
\text { simpsoni }\end{array}$ & $\begin{array}{l}\text { Santa Rosa (Yahuarango } \\
\text { Fm), Peru }\end{array}$ & $\begin{array}{l}\text { ?late Eocene / } \\
\text { early Oligocene }\end{array}$ & $\begin{array}{l}\text { INGEMMET } \\
\text { (holotype) }\end{array}$ & $\begin{array}{l}\text { Frailey \& Campbell } \\
2004\end{array}$ \\
\hline Eoespina-Eosachacui & E. woodi-E. lavocati & $\begin{array}{l}\text { Santa Rosa (Yahuarango } \\
\text { Fm), Peru }\end{array}$ & $\begin{array}{l}\text { ?late Eocene / } \\
\text { early Oligocene }\end{array}$ & $\begin{array}{l}\text { INGEMMET } \\
\text { (holotype) }\end{array}$ & $\begin{array}{l}\text { Frailey \& Campbell } \\
2004\end{array}$ \\
\hline Mayomys & M. confluens & $\begin{array}{l}\text { TAR-01, Shapaja (Pozo } \\
\text { Fm), Peru }\end{array}$ & early Oligocene & MUSM (original) & Boivin et al. 2018a \\
\hline Draconomys & D.verai & $\begin{array}{l}\text { La Cantera (Sarmiento Fm), } \\
\text { Argentina }\end{array}$ & $\begin{array}{l}\text {, late early } \\
\text { Oligocene }\end{array}$ & UM (cast) & Vucetich et al. 2010a \\
\hline Sallamys & S. pascuali & Salla (Salla Beds), Bolivia & $\begin{array}{l}\text { late early } \\
\text { Oligocene-- } \\
\text { late Oligocene }\end{array}$ & $\begin{array}{l}\text { MNHN-Bol, MNHN \& } \\
\text { UM (original) }\end{array}$ & $\begin{array}{l}\text { Hoffstetter \& Lavocat } \\
\text { 1970; Lavocat 1976; } \\
\text { Patterson \& Wood } \\
1982\end{array}$ \\
\hline Platypittamys & P. brachyodon & $\begin{array}{l}\text { Scaritt Pocket (Sarmiento } \\
\text { Fm), Argentina }\end{array}$ & late Oligocene & UM (cast) & $\begin{array}{l}\text { Wood 1949; Wood \& } \\
\quad \text { Patterson } 1959\end{array}$ \\
\hline Deseadomys & D. arambourgi & $\begin{array}{l}\text { Cabeza Blanca (Sarmiento } \\
\text { Fm) and La Flecha, } \\
\text { Argentina }\end{array}$ & $\begin{array}{l}\text { late early } \\
\text { Oligocene - } \\
\text { late Oligocene }\end{array}$ & MHNH (original) & $\begin{array}{l}\text { Wood \& Patterson } \\
1959\end{array}$ \\
\hline Loretomys & L. minutus & $\begin{array}{l}\text { Contamana CTA-32 } \\
\text { (Chambira Fm), Peru }\end{array}$ & late Oligocene & MUSM (original) & Boivin et al. 2017b \\
\hline Galileomys & G. eurygnathus & $\begin{array}{l}\text { Pinturas Fm (for the list of } \\
\text { localities, see Kramarz, } \\
\text { 2004), Argentina }\end{array}$ & late early Miocene & MACN (original) & Kramarz 2004 \\
\hline Dudumus & D. ruigomezi & $\begin{array}{l}\text { Bryn Gwyn (Sarmiento Fm), } \\
\text { Argentina }\end{array}$ & , early Miocene & MACN (original) & Arnal et al. 2014 \\
\hline Caviocricetus & C. lucasi & $\begin{array}{l}\text { Bryn Gwyn and Gran } \\
\text { Barranca (Sarmiento } \\
\text { Fm), Paso Córdoba } \\
\text { (Chinchinales Fm) and } \\
\text { Cerro Bandera (Cerro } \\
\text { Bandera Fm), Argentina }\end{array}$ & early Miocene & MACN (original) & $\begin{array}{l}\text { Vucetich \& Verzi 1996; } \\
\text { Vucetich et al. } \\
2010 \mathrm{~b}\end{array}$ \\
\hline Acaremys & A. murinus & $\begin{array}{l}\text { Sarmiento Fm, Pinturas } \\
\text { Fm and Santa Cruz Fm } \\
\text { (for the list of localities, } \\
\text { see Arnal and Vucetich, } \\
\text { 2015), Argentina }\end{array}$ & early Miocene & $\begin{array}{l}\text { MACN \& MLP } \\
\quad \text { (original) }\end{array}$ & $\begin{array}{l}\text { Ameghino 1887; } \\
\text { Arnal \& Vucetich } \\
\text { 2015b }\end{array}$ \\
\hline Willidewu & W. esteparius & $\begin{array}{l}\text { Paso Córdoba } \\
\text { (Chinchinales Fm) and } \\
\text { Bryn Gwyn (Sarmiento } \\
\text { Fm), Argentina }\end{array}$ & early Miocene & MLP (original \& cast) & $\begin{array}{l}\text { Vucetich \& Verzi 1991; } \\
\text { Vucetich et al. } \\
\text { 2010b }\end{array}$ \\
\hline Prospaniomys & $\begin{array}{l}\text { P. priscus- } \\
\text { P. cf. priscus }\end{array}$ & $\begin{array}{l}\text { Gran Barranca, Bryn } \\
\text { Gwyn?, Sacanana, } \\
\text { Pampa de Gran Gan } \\
\text { (also collected by } \\
\text { Ameghino; Sarmiento } \\
\text { Fm), Argentina }\end{array}$ & early Miocene & MACN (original) & $\begin{array}{l}\text { Ameghino 1902; } \\
\text { Vucetich et al. } \\
\text { 2010b; Arnal \& } \\
\text { Kramarz } 2011\end{array}$ \\
\hline Prostichomys & P. bowni & $\begin{array}{l}\text { Gran Barranca (Sarmiento } \\
\text { Fm) and Pinturas Fm (for } \\
\text { the list of localities, see } \\
\text { Kramarz, 2001, 2004), } \\
\text { Argentina }\end{array}$ & early Miocene & MACN (original) & $\begin{array}{r}\text { Kramarz 2001a, 2004; } \\
\text { Kramarz et al. } 2010\end{array}$ \\
\hline
\end{tabular}




\begin{tabular}{|c|c|c|c|c|c|}
\hline Genus & Species & $\begin{array}{l}\text { Locality and/or } \\
\text { Formation }\end{array}$ & Age & $\begin{array}{r}\text { Observed material } \\
\text { (original or cast) }\end{array}$ & Primary references \\
\hline Adelphomys & A. candidus & $\begin{array}{l}\text { Lago Cardiel and } \\
\text { Gobernador Gregores } \\
\text { (Pinturas Fm) and Santa } \\
\text { Cruz Fm, Argentina }\end{array}$ & early Miocene & $\begin{array}{l}\text { MACN \& MLP } \\
\quad \text { (original) }\end{array}$ & $\begin{array}{r}\text { Ameghino 1887; } \\
\text { Kramarz } 2004\end{array}$ \\
\hline Protadelphomys & P. latus & $\begin{array}{l}\text { Gran Brranca, Bryn Gwyn, } \\
\text { Sacanana (Sarmiento } \\
\text { Fm), Argentina }\end{array}$ & early Miocene & MLP (original) & $\begin{array}{l}\text { Ameghino 1902; } \\
\text { Vucetich \& Bond } \\
\text { 1984; Vucetich } \\
\text { et al. 1992, 2010b; } \\
\text { Vucetich \& Verzi } \\
\text { 1994 }\end{array}$ \\
\hline Spaniomys & S. riparius & $\begin{array}{l}\text { Santa Cruz Fm, Argentina; } \\
\text { Pampa Castillo } \\
\text { (unnamed Fm), Chile }\end{array}$ & \multicolumn{2}{|c|}{$\begin{array}{c}\text { late early MioceneMACN \& MLP } \\
\text { (original) }\end{array}$} & $\begin{array}{l}\text { Ameghino 1887, 1894; } \\
\text { Flynn et al. } 2002\end{array}$ \\
\hline Stichomys & S. regularis & Santa Cruz Fm, Argentina & \multirow{2}{*}{\multicolumn{2}{|c|}{$\begin{array}{c}\text { late early MioceneMACN (original) } \\
\text { late early MioceneMACN \& MLP } \\
\text { (original) }\end{array}$}} & Ameghino 1887 \\
\hline Sciamys & S. principalis & $\begin{array}{l}\text { Santa Cruz Fm, Argentina; } \\
\text { Pampa Castillo } \\
\text { (unnamed Fm), Chile }\end{array}$ & & & $\begin{array}{l}\text { Ameghino 1887; } \\
\text { Arnal \& Vucetich } \\
\text { 2011; Arnal \& Pérez } \\
\text { 2013; Flynn et al. } \\
\text { 2002 }\end{array}$ \\
\hline Plesiacarechimys & P. koenigswaldi & \multicolumn{2}{|c|}{$\begin{array}{l}\text { Estancia Cerro San Antonio middle Miocene } \\
\text { (Collón Cura Fm), } \\
\text { Argentina }\end{array}$} & MLP (original) & $\begin{array}{l}\text { Vucetich \& Vieytes } \\
\quad 2006\end{array}$ \\
\hline Maruchito & M. trilofodonte & $\begin{array}{l}\text { Canadon del Tordillo, } \\
\text { localities 1, } 4 \text { and } \\
5 \text { (Collón Cura Fm), } \\
\text { Argentina }\end{array}$ & middle Miocene & MLP (original) & Vucetich et al. 1993a \\
\hline Pampamys & P. emmonsae & $\begin{array}{l}\text { Cerro Azul Fm (for the list } \\
\text { of localities, see Olivares } \\
\text { et al., 2012), Argentina }\end{array}$ & \multicolumn{2}{|l|}{ late Miocene } & $\begin{array}{l}\text { Verzi et al. 1995; } \\
\quad \text { Olivares et al. } 2012\end{array}$ \\
\hline Xenodontomys & X. simpsoni-elongatus & $\begin{array}{l}\text { Barrancas Coloradas, El } \\
\text { Guanaco, Estancia, } \\
\text { El Rocado (Cerro Azul } \\
\text { Fm) and Salinas Chicas } \\
\text { basin (Los Salitrales Fm), } \\
\text { Argentina }\end{array}$ & \multicolumn{2}{|l|}{ late Miocene } & $\begin{array}{l}\text { Verzi 1999; Verzi et al. } \\
\text { 1991, } 2003\end{array}$ \\
\hline Neophanomys & N. biplicatus & $\begin{array}{l}\text { Chiquimil, Valle of } \\
\text { Santa María area and } \\
\text { Huayquerías de San } \\
\text { Carlos (unnamed Fm), } \\
\text { Argentina }\end{array}$ & \multicolumn{2}{|l|}{ late Miocene } & Verzi et al. 2011 \\
\hline Pithanotomys & P. columnaris & $\begin{array}{l}\text { Monte Hermoso Fm, } \\
\text { Argentina }\end{array}$ & early ?Pliocene & MACN (original) & \\
\hline Abrocoma & A. bennettii & & Recent & MNHN (original) & \\
\hline Spalacopus & S. cyanus & & Recent & MNHN (original) & \\
\hline Octodon & O. degus & & Recent & UM (original) & \\
\hline Ctenomys & C. boliviensis & & Recent & MLP \& UM (original) & \\
\hline $\begin{array}{l}\text { Mesocapromys/ } \\
\text { Mysasteles }\end{array}$ & $\begin{array}{l}\text { Mesocapromys/ } \\
\text { Mysasteles sp. }\end{array}$ & & Recent & UM (original) & \\
\hline Euryzygomatomys & E. spinosus & & Recent & $\begin{array}{l}\text { MLP \& MNHN } \\
\quad \text { (original) }\end{array}$ & \\
\hline Makalata & M. didelphoides & & Recent & UM (original) & \\
\hline Echimys & E. chrysurus & & Recent & UM (original) & \\
\hline Thrichomys & T. apereoides & & Recent & MNHN (original) & \\
\hline Proechimys & P. cuvieri & & Recent & UM (original) & \\
\hline Myocastor & M. coypus & & Recent & UM (original) & \\
\hline \multicolumn{6}{|l|}{ Erethizontoidea } \\
\hline Shapajamys & S. labocensis & $\begin{array}{l}\text { TAR-01, Shapaja (Pozo } \\
\text { Fm), Peru }\end{array}$ & early Oligocene & MUSM (original) & Boivin et al. 2018a \\
\hline Protosteiromys & P. medianus & $\begin{array}{l}\text { Cabeza Blanca and La } \\
\text { Flecha (Sarmiento Fm), } \\
\text { Argentina }\end{array}$ & $\begin{array}{l}\text { late early } \\
\text { Oligocene- } \\
\text { late Oligocene }\end{array}$ & $\begin{array}{l}\text { MACN \& MHNH } \\
\text { (original) }\end{array}$ & $\begin{array}{l}\text { Ameghino 1903; } \\
\text { Wood \& Patterson } \\
1959\end{array}$ \\
\hline Palaeosteiromys & P. amazonensis & $\begin{array}{l}\text { Contamana CTA-32 } \\
\text { (Chambira Fm), Peru }\end{array}$ & late Oligocene & MUSM (original) & Boivin et al. 2017b \\
\hline Plesiosteiromys & P. newelli & $\begin{array}{l}\text { Contamana CTA-61 } \\
\text { (Chambira Fm), Peru }\end{array}$ & late Oligocene & MUSM (original) & Boivin et al. 2017b \\
\hline
\end{tabular}


ANNEXE 3. - Suite.

\begin{tabular}{|c|c|c|c|c|c|}
\hline Genus & Species & $\begin{array}{l}\text { Locality and/or } \\
\text { Formation }\end{array}$ & Age & $\begin{array}{r}\text { Observed material } \\
\text { (original or cast) }\end{array}$ & Primary references \\
\hline Hypsosteiromys & H. axiculus & $\begin{array}{l}\text { Bryn Gwyn and Grand } \\
\text { Barranca (also collected } \\
\text { by Ameghino, Sarmiento } \\
\text { Fm), Argentina }\end{array}$ & early Miocene & MACN (original) & $\begin{array}{l}\text { Ameghino, 1902; } \\
\text { Candela 2000; } \\
\text { Candela \& Vucetich } \\
\text { 2002; Vucetich et al. } \\
\text { 2010b }\end{array}$ \\
\hline Steiromys & S. detentus & Santa Cruz Fm, Argentina & early Miocene & $\begin{array}{l}\text { MACN \& MLP } \\
\quad \text { (original) }\end{array}$ & $\begin{array}{l}\text { Ameghino 1887; } \\
\text { Candela 2000; } \\
\text { Candela et al. 2012; } \\
\text { Kay et al. } 2012\end{array}$ \\
\hline Eosteiromys & E. homogenidens & $\begin{array}{l}\text { Bryn Gwyn and Grand } \\
\text { Barranca (also collected } \\
\text { by Ameghino, Sarmiento } \\
\text { Fm), Argentina }\end{array}$ & early Miocene & MACN \& MLP (original & $\begin{array}{l}\text { Ameghino 1902; } \\
\text { Candela 2000; } \\
\text { Vucetich et al. } \\
\text { 2010b }\end{array}$ \\
\hline Branisamyopsis & $\begin{array}{l}\text { B. australis- } \\
\text { praesigmoides }\end{array}$ & $\begin{array}{l}\text { Southern Cliff of Lake } \\
\text { Colhué Huapi (Gran } \\
\text { Barranca; Sarmiento } \\
\text { Fm), Argentina; Pinturas } \\
\text { Fm (for the list of } \\
\text { localities, see Kramarz, } \\
\text { 2004), Argentina }\end{array}$ & early Miocene & $\begin{array}{l}\text { MACN, MLP \& MHNH } \\
\text { (original) }\end{array}$ & $\begin{array}{l}\text { Candela 2000, 2003; } \\
\text { Kramarz 2004; } \\
\text { Vucetich et al. } \\
\text { 2010b }\end{array}$ \\
\hline Neosteiromys & N. pattoni & $\begin{array}{l}\text { Chiquimil (Andalhuala Fm), } \\
\text { Argentina }\end{array}$ & late Miocene & & Candela 2000, 2004 \\
\hline Coendou & $\begin{array}{l}\text { C. prehensilis- } \\
\text { insidiosus }\end{array}$ & & Recent & $\begin{array}{l}\text { MLP \& MNHN } \\
\quad \text { (original) }\end{array}$ & Candela 1999 \\
\hline Erethizon & E. dorsatum & & Recent & MNHN (original) & \\
\hline Chaetomys & C. subspinosus & & Recent & MHNG (pictures) & Martin 1994 \\
\hline \multicolumn{6}{|l|}{$\begin{array}{l}\text { Chinchilloidea or } \\
\text { Cavioidea }\end{array}$} \\
\hline Branisamys & B. luribayensis & Salla (Salla Beds), Bolivia & $\begin{array}{l}\text { late early } \\
\text { Oligocene - } \\
\text { late Oligocene }\end{array}$ & $\begin{array}{l}\text { MNHN-Bol, MNHN \& } \\
\text { UM (original) }\end{array}$ & $\begin{array}{l}\text { Hoffstetter \& Lavocat } \\
\text { 1970; Lavocat 1976; } \\
\text { Patterson \& Wood } \\
1982\end{array}$ \\
\hline \multicolumn{6}{|l|}{ Chinchilloidea } \\
\hline Incamys & I. bolivianus & $\begin{array}{l}\text { Salla (Salla Beds), } \\
\text { Bolivia; Cabeza Blanca } \\
\text { (Sarmiento Fm), } \\
\text { Argentina }\end{array}$ & $\begin{array}{l}\text { late early } \\
\text { Oligocene-- } \\
\text { late Oligocene }\end{array}$ & $\begin{array}{l}\text { MNHN-Bol, MNHN \& } \\
\text { UM (original) }\end{array}$ & $\begin{array}{l}\text { Hoffstetter \& Lavocat } \\
\text { 1970; Lavocat 1976; } \\
\text { Patterson \& Wood } \\
\text { 1982; Busker \& } \\
\text { Dozo } 2017\end{array}$ \\
\hline \multirow[t]{2}{*}{ Eoviscaccia } & E. frassinettii & $\begin{array}{l}\text { Tinguiririca Termas del } \\
\text { Flaco (Abanico Fm), } \\
\text { Chile }\end{array}$ & $\begin{array}{l}\text { late early } \\
\text { Oligocene-- } \\
\text { late Oligocene }\end{array}$ & & $\begin{array}{l}\text { Wyss et al. 1993; } \\
\text { Bertrand et al. } 2012\end{array}$ \\
\hline & E. australis & $\begin{array}{l}\text { Sarmiento Fm, Chichinales } \\
\text { Fm and Cerro Bandera } \\
\text { Fm, Argentina (for the list } \\
\text { of localities, see Vucetich } \\
\text { et al., 2010b) }\end{array}$ & early Miocene & MACN (original) & $\begin{array}{l}\text { Vucetich 1989; } \\
\text { Kramarz 2001b; } \\
\text { Vucetich et al. } \\
\text { 2010b }\end{array}$ \\
\hline Garridomys & G. curunuquem & $\begin{array}{l}\text { near Barda Negra (Cerro } \\
\text { Bandera Formation), } \\
\text { Argentina }\end{array}$ & early Miocene & & Kramarz et al. 2013 \\
\hline \multirow[t]{4}{*}{ Scleromys } & S. praecursor & $\begin{array}{l}\text { Contamana CTA-61 } \\
\text { (Chambira Fm), Peru }\end{array}$ & late Oligocene & MUSM (original) & Boivin et al. 2017b \\
\hline & S. quadrangulatus & $\begin{array}{l}\text { Pinturas Fm (for the list of } \\
\text { localities, see Kramarz, } \\
\text { 2006), Argentina }\end{array}$ & $\begin{array}{l}\text { late early } \\
\text { Miocene }\end{array}$ & $\begin{array}{l}\text { MACN \& MLP } \\
\text { (original) }\end{array}$ & Kramarz 2006b \\
\hline & S. angustus & Santa Cruz Fm, Argentina & $\begin{array}{l}\text { late early } \\
\text { Miocene }\end{array}$ & MACN (original) & Ameghino 1887 \\
\hline & S. osbornianus & $\begin{array}{l}\text { Santa Cruz Fm and } \\
\text { Pinturas Fm, Argentina }\end{array}$ & $\begin{array}{l}\text { late early } \\
\text { Miocene }\end{array}$ & MACN (original) & $\begin{array}{l}\text { Ameghino 1894; } \\
\text { Kramarz 2006b }\end{array}$ \\
\hline Perimys & P. intermedius & $\begin{array}{l}\text { Pinturas Fm (for the list of } \\
\text { localities, see Kramarz, } \\
\text { 2002), Argentina }\end{array}$ & late early Miocene & & Kramarz 2002 \\
\hline Drytomomys & D. aequatorialis & $\begin{array}{l}\text { near the hacienda of Seňor } \\
\text { Carrasco (near Nabon), } \\
\text { Ecuador; La Venta } \\
\text { (Villavieja Fm), Colombia }\end{array}$ & $\begin{array}{l}\text { late middle } \\
\text { Miocene }\end{array}$ & & $\begin{array}{l}\text { Anthony 1922; Fields } \\
\text { 1957; Walton 1997; } \\
\text { Candela \& Nasif } \\
2006\end{array}$ \\
\hline
\end{tabular}


ANNEXE 3. - Suite.

\begin{tabular}{|c|c|c|c|c|c|}
\hline Genus & Species & $\begin{array}{l}\text { Locality and/or } \\
\text { Formation }\end{array}$ & Age & $\begin{array}{r}\text { Observed material } \\
\text { (original or cast) }\end{array}$ & Primary references \\
\hline Potamarchus & P. murinus & $\begin{array}{l}\text { Solimoes Fm (for the list } \\
\text { of localities, see Kerber } \\
\text { et al., 2016), Brazil }\end{array}$ & late Miocene & & Kerber et al. 2016 \\
\hline Neoepiblema & $\begin{array}{l}\text { N. ambrosettianus- } \\
\text { horridula }\end{array}$ & $\begin{array}{l}\text { Niteroi and Cachoeira } \\
\text { do Bandeiro localities } \\
\text { (Solimoes Fm), Brazil }\end{array}$ & late Miocene & UFAC (original) & Kerber et al. 2017 \\
\hline Chinchilla & C. lanigera & & Recent & MLP (original) & \\
\hline Lagidium & L. viscacia & & Recent & $\begin{array}{l}\text { MLP \& MNHN } \\
\text { (original) }\end{array}$ & \\
\hline Lagostomus & L. maximus & & Recent & $\begin{array}{l}\text { MLP \& MNHN } \\
\text { (original) }\end{array}$ & \\
\hline Dinomys & D. branickii & & Recent & MNHN (original) & \\
\hline \multicolumn{6}{|l|}{ ?Chinchilloidea } \\
\hline \multirow[t]{4}{*}{ Eoincamys } & E. ameghinoi & $\begin{array}{l}\text { Santa Rosa (Yahuarango } \\
\text { Fm), Peru }\end{array}$ & $\begin{array}{l}\text { ?late Eocene/ } \\
\quad \text { early Oligocene }\end{array}$ & INGEMMET (holotype) & $\begin{array}{l}\text { Frailey \& Campbell } \\
2004\end{array}$ \\
\hline & E. pascuali & $\begin{array}{l}\text { Santa Rosa (Yahuarango } \\
\text { Fm), Peru }\end{array}$ & $\begin{array}{l}\text { ?late Eocene/ } \\
\quad \text { early Oligocene }\end{array}$ & INGEMMET (holotype) & $\begin{array}{l}\text { Frailey \& Campbell } \\
2004\end{array}$ \\
\hline & E. valverdei & $\begin{array}{l}\text { TAR-13, TAR-20-22, } \\
\text { Shapaja (Pozo Fm), Peru }\end{array}$ & early Oligocene & MUSM (original) & Boivin et al. 2018a \\
\hline & E. parvus & $\begin{array}{l}\text { TAR-20-22, Shapaja (Pozo } \\
\text { Fm), Peru }\end{array}$ & early Oligocene & MUSM (original) & Boivin et al. 2018a \\
\hline \multicolumn{6}{|l|}{ Cavioidea } \\
\hline \multirow[t]{2}{*}{ Eobranisamys } & E. javierpradoi & $\begin{array}{l}\text { Contamana CTA-27 (Pozo } \\
\text { Fm), Peru }\end{array}$ & $\begin{array}{l}\text { late middle } \\
\text { Eocene }\end{array}$ & MUSM (original) & $\begin{array}{l}\text { Antoine et al. 2012; } \\
\text { Boivin et al. 2017a }\end{array}$ \\
\hline & E. romeropittmanae & $\begin{array}{l}\text { Santa Rosa (Yahuarango } \\
\text { Fm), Peru }\end{array}$ & $\begin{array}{l}\text { ?late Eocene / } \\
\text { early Oligocene }\end{array}$ & INGEMMET (holotype) & $\begin{array}{l}\text { Frailey \& Campbell } \\
\quad 2004\end{array}$ \\
\hline Asteromys & A. punctus & $\begin{array}{l}\text { Cabeza Blanca (Sarmiento } \\
\text { Fm), Argentina }\end{array}$ & $\begin{array}{l}\text { late early } \\
\text { Oligocene - } \\
\text { late Oligocene }\end{array}$ & MACN (original) & $\begin{array}{l}\text { Ameghino 1887; } \\
\text { Wood \& Patterson } \\
\text { 1959; Pérez \& } \\
\text { Vucetich 2012b }\end{array}$ \\
\hline Australoprocta & A. fleaglei & $\begin{array}{l}\text { Bryn Gwyn, Sacanana } \\
\text { (Sarmineto Fm) and Paso } \\
\text { Córdoba (Chinchinales } \\
\text { Fm), Argentina }\end{array}$ & early Miocene & & $\begin{array}{l}\text { Kramarz 1998; } \\
\text { Vucetich et al. } \\
\text { 2010b }\end{array}$ \\
\hline Neoreomys & N. australis & $\begin{array}{l}\text { Santa Cruz Fm and } \\
\text { Pinturas Fm (for the list } \\
\text { of localities, see Kramarz } \\
\text { 2006b), Argentina; } \\
\text { Pampa Castillo } \\
\text { (unnamed Fm), Chile }\end{array}$ & $\begin{array}{l}\text { late early } \\
\text { Miocene }\end{array}$ & $\begin{array}{l}\text { MACN \& MLP } \\
\text { (original) }\end{array}$ & $\begin{array}{l}\text { Ameghino 1887; } \\
\text { Kramarz 2006b; } \\
\text { Flynn et al. 2002; } \\
\text { Candela et al. 2012; } \\
\text { Kay et al. } 2012\end{array}$ \\
\hline Luantus & L. propheticus & $\begin{array}{l}\text { Pinturas Fm (for the list of } \\
\text { localities, see Kramarz, } \\
\text { 2006), Argentina }\end{array}$ & late early Miocene & & Kramarz 2006a \\
\hline Microscleromys & $\begin{array}{l}\text { M. paradoxalis- } \\
\text { cribriphilus }\end{array}$ & $\begin{array}{l}\text { La Venta (Villavieja Fm), } \\
\text { Colombia }\end{array}$ & $\begin{array}{r}\text { late middle } \\
\text { Miocene }\end{array}$ & & Walton 1990, 1997 \\
\hline Dasyprocta & D. sp. & & Recent & MLP \& UM (original) & \\
\hline Cuniculus & C. paca-taczanowskii & & Recent & UM (original) & \\
\hline Cavia & C. aperea & & Recent & MLP (original) & \\
\hline Galea & G. spixii & & Recent & MNHN (original) & \\
\hline Dolichotis & D. patagonum & & Recent & UM (original) & \\
\hline Hydrochoerus & H. hydrochaeris & & Recent & $\begin{array}{l}\text { MLP \& MNHN } \\
\text { (original) }\end{array}$ & \\
\hline \multicolumn{6}{|c|}{$\begin{array}{l}\text { Incertae sedis extinct } \\
\text { superfamily }\end{array}$} \\
\hline Litodontomys & L. chubutomys & $\begin{array}{l}\text { Cabeza Blanca (Sarmiento } \\
\text { Fm), Argentina }\end{array}$ & $\begin{array}{l}\text { late early } \\
\text { Oligocene - } \\
\text { late Oligocene }\end{array}$ & & $\begin{array}{l}\text { Loomis 1914; Wood \& } \\
\text { Patterson } 1959\end{array}$ \\
\hline Cephalomys & C. arcidens & $\begin{array}{l}\text { Cabeza Blanca and La } \\
\text { Flecha (Sarmiento Fm), } \\
\text { Argentina }\end{array}$ & $\begin{array}{l}\text { late early } \\
\text { Oligocene - } \\
\text { late Oligocene }\end{array}$ & MNHN (original) & $\begin{array}{l}\text { Ameghino 1897; } \\
\text { Wood \& Patterson } \\
1959\end{array}$ \\
\hline «Asteromys » & «A. » bolivianus & Salla (Salla Beds), Bolivia & $\begin{array}{l}\text { late early } \\
\text { Oligocene - } \\
\text { late Oligocene }\end{array}$ & $\begin{array}{l}\text { MNHN-Bol, MNHN \& } \\
\text { UM (original) }\end{array}$ & $\begin{array}{l}\text { Lavocat 1976; } \\
\text { Patterson \& Wood } \\
\text { 1982; Pérez et al. } \\
2018\end{array}$ \\
\hline Soriamys & S. gaimanensis & $\begin{array}{l}\text { Bryn Gwyn (Sarmiento Fm), } \\
\text { Argentina }\end{array}$ & early Miocene & & $\begin{array}{l}\text { Kramarz 2001c; } \\
\text { Vucetich et al. } \\
2010 b\end{array}$ \\
\hline
\end{tabular}


ANNEXE 4. - Consensus stricts obtenus pour les AG1 non équilibré et AG2-AG9.

AG1 non équilibrée
872 arbres équiparcimonieux
3839 pas chacun
IC $=0,547$
IR $=0,593$


ANNEXE 4. - Consensus stricts obtenus pour les AG1 non équilibré et AG2-AG9 (suite).

AG2 : inclusion de Gaudeamus aslius, $G$. hylaeus et $G$. aegyptius

42 arbres équiparcimonieux 3493,48 pas

$\mathrm{IC}=0,582$

$\mathrm{IR}=0,597$
Spaniomys riparius Myocastor coypus Proechimys cuvier Maruchito trilofodonte Thrichomys apereoides Echimys chrysurus Makalata didelphoides Mesocapromys/Mysateles sp. Xenodontomys simpsoni-elongatus Ctenomys boliviensis Neophanomys biplicatus Pithanotomys columnaris Octodon degus Spalacopus cyanus Abrocoma bennettii Prostichomys bowni Stichomys regularis Adelphomys candidus Prospaniomys priscus-cf. priscus Dudumus ruigomezi Pampamys emmonsa Plesiacarechimys koenigwaldi Acaremys murinus Sciamys principalis Galileomys eurygnathus Platypittamys brachyodon Deseadomys arambourgi Willidewu esteparius Sallamys pascuali Draconomys verai Leucokephalos zeffiae Mayomys confluens Chinchilla lanigera
Lagidium viscacia Lagostomus maximus Neoepiblema ambrosettianus-horridula Dinomys branickil Drytomomys aequatorialis Potamarchus murinus Eoviscaccia frassinet Eoviscaccia australis Gcleromys quadrangulatus Scleromys angustus Scleromys osbornianus Incamys bolivianus Scleromys praecursor Chambiramys shipibor Gaudeamus aslius Gaudeamus aegyptius Gaudeamus hylaeus Eoincamys pascuali Eoincamys parvus Microscleromys paradoxalis-cribriphilus Chambiramys sylvaticus Eoincamys valverdei Eoincamys ameghino Eosallamys paulacoutoi-simpsoni Dolichotis patagonum haeris Cavia aperea

Perimys intermedius Asteromys punctus Luantus propheticus Australoprocta fleagi Neoreomys australis Branisamys luribayensis Dasyprocta sp. Cuniculus paca-taczanowski Branisamyopsis australis-praesigmoides Neosteiromys pattoni

Eosteiromys homogenidens

Erethizon prehensilis-insidiosus Erethizon dorsatum Protosteiromys medianus Hypsosteiromys axiculus Chaetomys subspinosus Palaeosteiromys amazonensis Eobranisamys romeropittmana Eobranisamys javierpradol Shapajamys labocensis Tarapotomys mayoensis Tarapotomys subandinus Pozomys ucayaliensis Eoespina woodi-Eosachacui lavocati Canaanimys maquiensis Cachiyacuy kummeli Phiomys hammuda Thryonomys swinderianus Paraphiomys pigotti Metaphiomys schaubi Acritophiomys bowni Bugtimys zafarullahi Hodsahibia azra 'Waslamys' attiai Ottomania proavita Baluchimys barryi

Tamquammys dispinorum-wilsoni-tantillus Reithroparamys delicatissimus Birbalomys sondaari-woodi Brrbalomys sondaari-woodis Tribosphenomys minutus Cocomys ligchaensis 
ANNEXE 4. - Consensus stricts obtenus pour les AG1 non équilibré et AG2-AG9 (suite).

AG3 : inclusion d'« Asteromys » bolivianus, de Cephalomys arcidens, Litodontomys et Soriamys non contraints

2 arbres équiparcimonieux 3393,98 pas

$\mathrm{IC}=0,562$

$\mathrm{IR}=0,592$

- Spaniomys riparius Myocastor coypus Maruchito trilofodonte

- Thrichomys apereoides

- Echimys chrysurus

Makalata didelphoides

- Mesocapromys/Mysateles sp.

Euryzygomatomys spinosus

- Xenodontomys simpsoni-

- Neophanomys biplicatus

- Octodon degus

- Abrocoma bennettii

- Prostichomys bowni

Stichomys regularis

Adelphomys candidus

Prospaniomys priscus-cf. priscus

Dudumus ruigomezi

Plesiacarechimys koenigwaldi

- Acaremys murinus

Acaremys murinus

Galileomys eurygnathus

- Leucokephalos zeffiae

Willidewu esteparius

Deseadomys arambourgi

Platypittamys brachyodon

- C Chinchilla lanigera

Lagidium viscacia

Neoepiblema ambrosettianus-horridula

Dinomys branickii

Drytomomys aequatorialis

Potamarchus murinus

- Eoviscaccia frassinetti

- Scleromys quadrangulatus

Garridomys curnunuquem

Scleromys angustus

Scleromys osbornianu

Incamys bolivianus

- Chambiramys shipiborum

Eoincamys pascuali

Microscleromys paradoxalis-cribriphilus

Chambiramys sylvaticus

Protadelphomys latus

- Cephalomys arcidens

"Asteromys " bolivianus

Litodontomys chubutomys

Asteromys punctus

Eoincamys valverdei

Eoincamys ameghinoi

Eoespina woodi-Eosachacui lavocati

Mayomys confluens

Tarapotomys mayoensis

Tarapotomys subandinus

Pozomys ucayaliensis

- Eobranisamys romeropittmanae

Eobranisamys javierpradoi

Shapajamys labocensis

- Cachiyacuy contaman

Cachiyacuy kummeli

- Hydrochoerus hydrochaeris

- Gavia aperea spixii

Perimys intermedius

Soriamys gain

Luantus propheticus

Neoreomys australis

Australoprocta fleagi

Branisamys luribayensis

- Dasyprocta sp.

- Cuniculus paca-taczanowskii

Pranisamyopsis australis-pris

- Neosteiromys pattoni

Steiromys detentus

Erethizon dorsatum

- Coendou prehensilis-insidiosus

- Protosteiromys medianus

Eosteiromys homogeniden

Hypsosteiromys axiculus

Chaetomys subspinosus

Draconomys verai

- Paraphiomys pinderianus

Paraphiomys pigotti

Metaphiomys schaubi

Acritophiomys bowni

Bugtimys zafarullahi

Hodsahibia azrae

'Waslamys' attiai

Ottomania proavita

Baluchimys barryi

Chapattimys wilsoni

Reithroparamys delicatissimus

Reithroparamys delicatissim
Birbalomys sondaari-woodi
Archetypomys erlianensis

Tribosphenomys minutus

Cocomys ligchaensis 
ANNEXE 4. - Consensus stricts obtenus pour les AG1 non équilibré et AG2-AG9 (suite).

AG4 : inclusion d'“ Asteromys " bolivianus, A. punctus, de $C$. arcidens, Litodontomys et Soriamys contraints en un clade divergeant dans les chinchilloïdes

291 arbres équiparcimonieux

3397,47 pas

IC $=0,511$

$\mathrm{IR}=0,591$
- Chinchilla lanigera

Neoepiblema ambrosettianus-horridula

Lagostomus maximus

Drytomomys aequatorials

Potamarchus murinus

Dinomys branickil

Scleromys quadrangulatus

Scleromys osbornianus

- Elovisins angustus

Eoviscaccia frassinetti

Garridomys curnunuquem

Eoincamys pascuali

Eoincamys parvus

Incamys bolivianus

Scleromys praecurso

Microscleromys paradoxalis-cribriphilus

Chambiramys shipiborum

Chambiramys sylvaticus

- Eoincamys ameghino

Protanys valverdei

Protadelphomys latus

- Cephalomys arcidens

Litodontomys chubutomys

Asteromys punctus

Soriamys gaimanensi

Spaniomys riparius

Myocastor coypus

Proechimys cuvieri

Maruchito trilofodonte

- Pampamys emmonsae

- Echimys chrysurus

- Echimys chrysurus

- Mesocapromys/Mysateles sp.

- Mesocapromys/Mysateles sp.

- Xenodontomys simpsoni-elongatus

- Ctenomys boliviensis

- Neophanomys biplicatus

- Pithanotomys columnaris

Octodon degus

Spalacopus cyanus

Abrocoma bennetti

- Prostichomys bown

Stichomys regularis

Prospaniomys priscus-cf. priscus

Dudumus ruigomez

Acaremys murinus

Sciamys principalis

Caviocricetus lucasi

Plesiacarechimys koenigwald

Galileomys eurygnathus

Draconomys veral

Platypittamys brachyodon

Sallamys pascuali

Willidewu esteparius

Deseadomys arambour

Leucokephalos zeffiae

Hydrochoerus hydrochaeris

Cavia aperea

Galea spixii

Perimys intermedius

Luantus propheticus

Australoprocta fleagi

Neoreomys australis

Branisamys luribayensis

- Dasyprocta sp.

paca-taczanowsk il

(

Branisamyopsis australis-praesigmoides

Neosteiromys pattoni

Eosteiromys homogeniden

Erethizon dorsatum

Coendou prehensilis-insidiosus

Steiromys detentus

Protosteiromys medianus

Hypsosteiromys axiculus

Chaetomys subspinosus

Eobranisamys romeropittmanae

Eobranisamys javierprado

Shapajamys labocensis
Eosallamys paulacoutoi-simpsoni

Eosallamys paulacouto-s

Tazomys ucayaliensis

Mayomys confluens

Tarapotomys subandinus

Cachiyacuy contamanensis

Canaanimys maquiensis

Eoespina woodi-Eosachacui lavocat

Cachiyacuy kummel

Phiomys hammudai
Thryonomys swinderianus

Thryonomys swinderi

Paraphiomys pigotti

Acritophiomys bowni

Acritophiomys bowni

Hodsahibia azrae

'Waslamys' attiai

Ottomania proavita

Baluchimys barryi

Chapattimys wilsoni
Tamquammys dispinorum-wilsoni-tantillus

Reithroparamys delicatissimus

Birbalomys sondaari-woodi

Archetypomys erlianensis

Tribosphenomys minutu 


AG5 : inclusion d'« Asteromys » bolivianus, A. punctus,
de $C$. arcidens, Litodontomys et Soriamys contraints
en un clade divergeant dans les cavioïdes
6 arbres équiparcimonieux
3398,14 pas
IC $=0,561$
IR $=0,591$

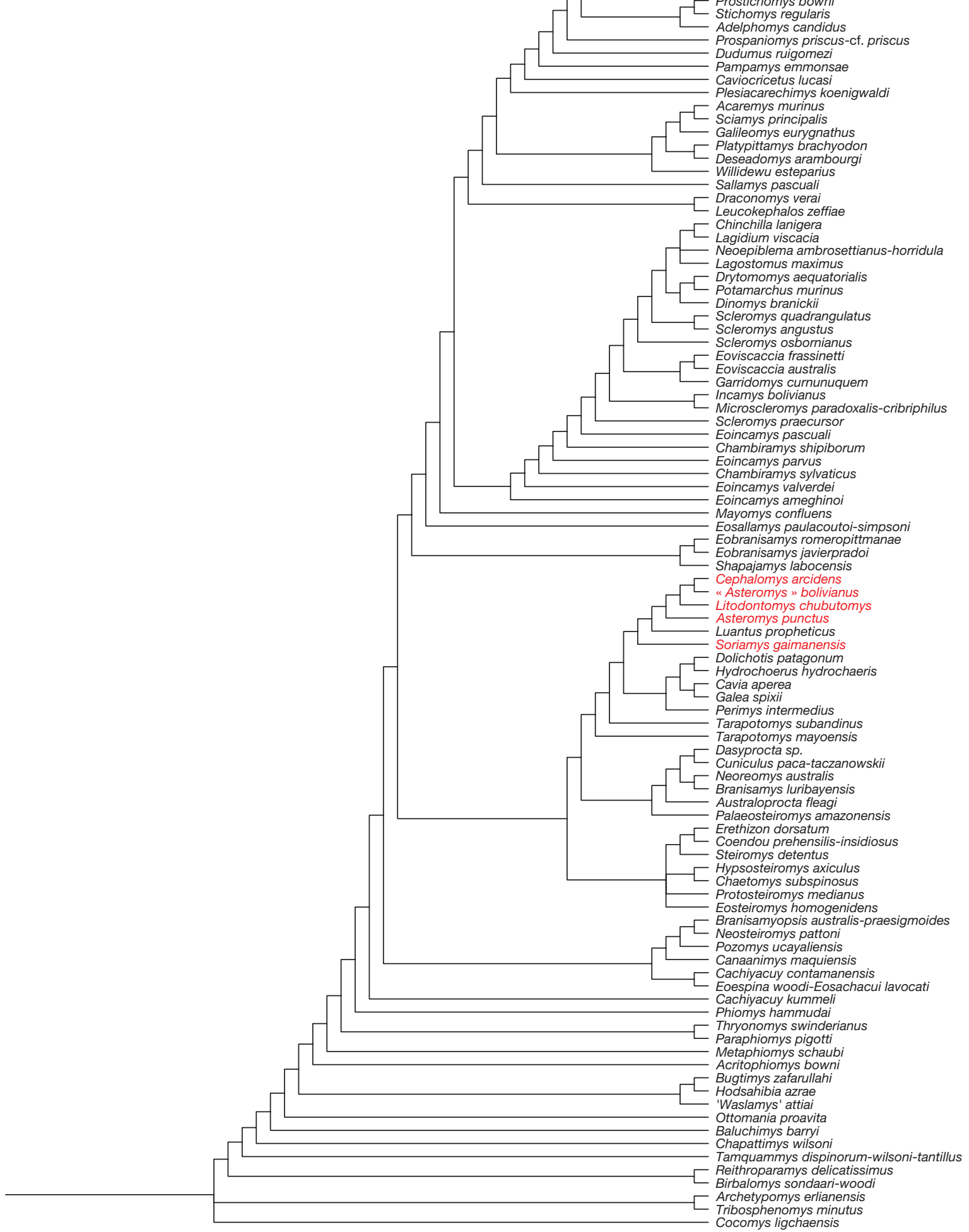


ANNEXE 4. - Consensus stricts obtenus pour les AG1 non équilibré et AG2-AG9 (suite).

AG6 : inclusion d' "Asteromys » bolivianus, de $C$. arcidens et Litodontomys non contraints

4 arbres équiparcimonieux

3372,97 pas

$\mathrm{IC}=0,565$

$\mathrm{IR}=0,594$
- Spaniomys riparius Proechimys cuviei Maruchito trilofodonte Thrichomys apereoides Echimys chrysurus

Makalata didelphoides

- - Protadelphomys latus

Euryzygomatomys spinosus

Xenodontomys simpsoni-elongatus

- Ctenomys boliviensis

Neophanomys biplicatus

Pithanotomys columnaris

Octodon degus

Spalacopus cyanus

Abrocoma bennettii

Prostichomys bowni

Stichomys regularis

Adelphomys candidus

Dudumus ruigomezi

Pampamys emmonsa

Caviocricetus lucasi

Plesiacarechimys koer

Sciamys principalis

Galileomys eurygnathus

- Platypittamys brachyodon

Deseadomys arambourgi

Willidewu esteparius

Sallamys pascuali

Draconomys verai

Chinchilla lanigera

Lagidium viscacia

Neoepiblema ambrosettianus-horridula

Lagostomus maximus

Drytomomys aequatorialis

Potamarchus murinus

Dinomys branickii

Scleromys quadrangulatus

Scleromys angustus

- Eoviscaccia australis

Garridomys curnunuquem

Incamys bolivianus

Microscleromys paradoxalis-cribriphilus

Eoincamys pascuali

Chambiramys shipibo

Eoincamys parvus

Chambiramys sylvaticus

Eoincamys valverdei

Eoincamys ameghinoi

Mayomys confluens

Canaanimys maquien

ropittmanae

Eobranisamys javierprado

Shapajamys labocensis

Dolichotis patagonum

Cavia aperea

Galea spixii

Perimys intermedius

Cephalomys arcidens

"Asteromys " bolivianus

Litodontomys chabutomys

Asteromys punctus

Tarapotomys subandinus

Pozomys ucayaliensis

Tarapotomys mayoensis

Australoprocta fleagi

Branisamys luribayensis

Dasyprocta sp.
Cuniculus paca-taczanowskil

Neoreomys australis

Palaeosteiromys amazonensis

- - Branisamyopsis austrilis

Erethizon dorsatum

Coendou prehensilis-insidiosus

Hypsosteiromys axiculus

Chaetomys subspinosus

Protosteiromys medianus

Eosteiromys homogenidens

Cachiyacuy contamanensis

Eosachacui lavocati

Phiomys hammudai

Phyonomys swinderianus

Paraphiomys pigotti

Metaphiomys schaubi

Acritophiomys bowni

Hodsahibia azrae

'Waslamys' attiai

Ottomania proavita

Baluchimys barryi

Chapattimys wilsoni

Tamquammys dispinorum-wilsoni-tantillus

Reithroparamys delicatissimus

Birbalomys sondaari-woodi

Archetypomys erlianensis

Tribosphenomys minutus 


AG7 : inclusion d'« Asteromys » bolivianus,
A. punctus, de C. arcidens et Litodontomys
contraints en un clade divergeant dans
les chinchilloïdes
52 arbres équiparcimonieux
3373,31 pas
IC $=0,565$
IR $=0,594$

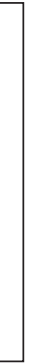

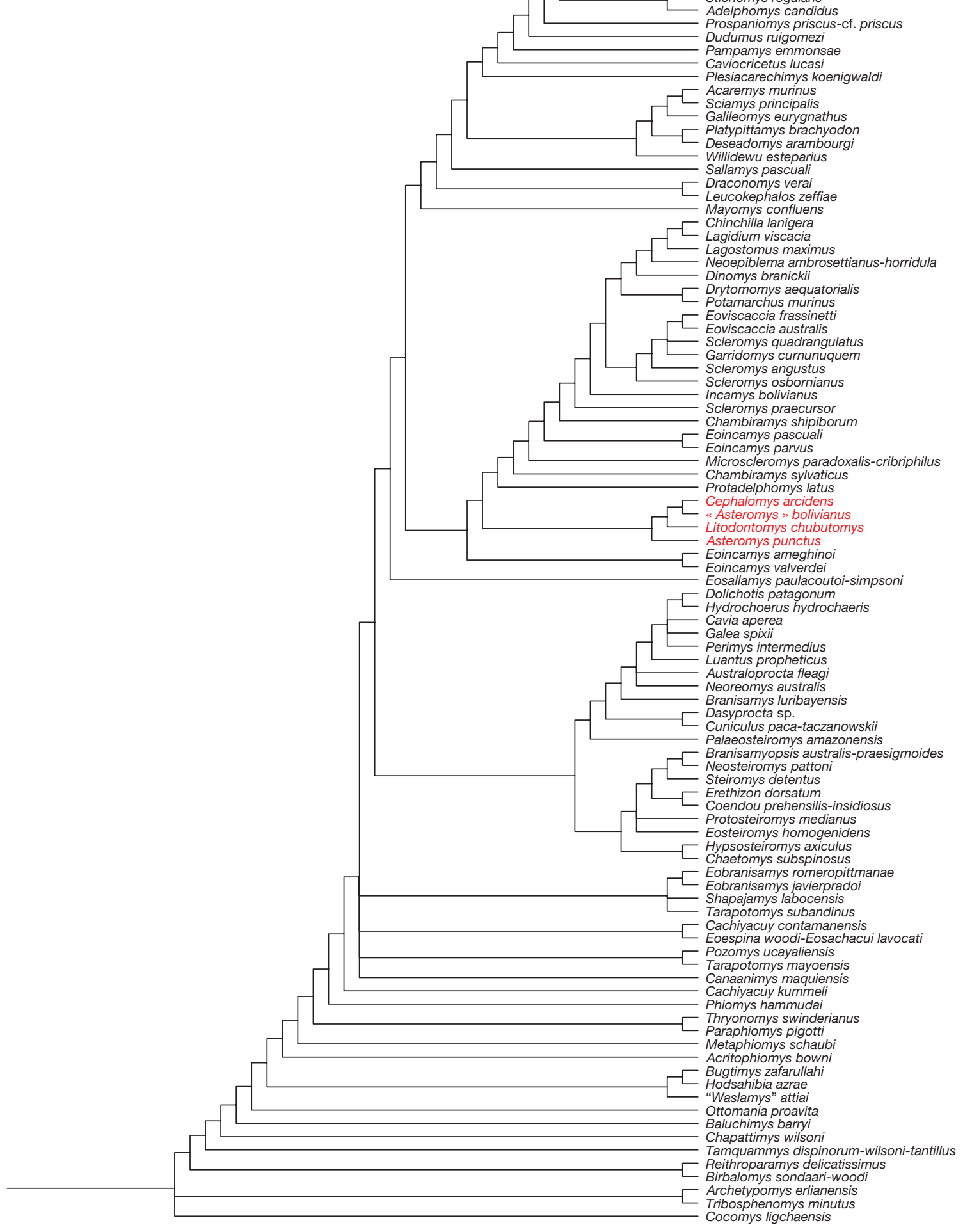


ANNEXE 4. - Consensus stricts obtenus pour les AG1 non équilibré et AG2-AG9 (suite).

\section{AG8 : inclusion de Plesiosteiromys}

16 arbres équiparcimonieux 3304,98 pas

$\mathrm{IC}=0,573$

$\mathrm{IR}=0,599$
- Spaniomys riparius Myocastor coypus Maruchito trilofodont Thrichomys apereoides Echimys chrysurus Makalata didelphoides Protadelphomys latus Euryzygomatomys spinosus Mesocapromys/Mysateles sp. Xenodontomys simpsoni-elongatus Ctenomys boliviensis Neophanomys biplicatus Pithanotomys columnan Sptodon degus

Spalacopus cyanus

Prostichomys bown

Stichomys regularis

Adelphomys candidus

Prospaniomys priscus-cf. priscus

Dudumus ruigomezi

Pampamys emmonsae

Caviocricetus lucasi

Plesiacarechimys koenigwaldi

Acaremys murinus

Sciamys principalis

Galileomys eurygnathus

Platypittamys brachyodon

Willidemu aramius

Willidewu esteparius

Draconomys verai

Leucokephalos zeffia

Chinchilla lanigera

Lagidium viscacia

Neoepiblema ambrosettianus-horridula

Lagostomus maximus

Drytomomys aequatorialis

Potamarchus murinus

Dinomys branickii

Scleromys quadrangulatus

Scleromys angustus

Scleromys osbornianus

Eoviscaccia frassinetti

Garridomys curnunuquem

Gncamys bolivianus

Incamys bolivianus

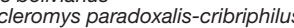

Scleromys praecursor

Chambiramys shipiborum

Eoincamys parvus

Chambiramys sylvaticus

Eoincamys valverdei

Eoincamys ameghino

Mayomys confluens

Eosallamys paulacoutoi-simpsoni

Dolichotis patagonum

chaeris

Cavia aperea

Perimys intermedius

Asteromys punctus

Luantus propheticus

Australoprocta fleagi

Neoreomys australis

Branisamys luribayensis

Dasyprocta sp.

Cuniculus paca-taczanowskil

Branisamyopsis australis-praesigmoide

Neosteiromys pattoni

Eosteiromys homogeniden

Erethizon dorsatum

Coendou prensilis-insidiosus

Steiromys detentus

Protosteiromys medianus

Hypsosteiromys axiculus

Palaeosteiromys amazonensis

Eobranisamys romeropittmana

Eobranisamys javierpradoi

Shapajamys labocensis

Plesiosteiromys newelli

Tarapotomys subandinus

Pozomys ucayaliensis

Tarapotomys mayoensis

Cachiyacuy contamanensis

Eoespina woodi-Eosachacui lavocati

Canaanimys maquiensis

Cachiyacuy kumme

Thryonomys swinderianus

Thryonomys swinderi

Paraphiomys pigotti

Metaphiomys schaubi

Acritophiomys bowni

Hodsahibia azrae

'Waslamys' attiai

Ottomania proavita

Baluchimys barryi

Chapattimys wilsoni

Tamquammys dispinorum-wilsoni-tantillus

Reithroparamys delicatissimus

Birbalomys sondaari-woodi

Archetypomys erlianensis

Cocomys ligchaensis 


\section{AG9 : inclusion de Loretomys}

54 arbres équiparcimonieux 3308,15 pas chacun

IC $=0,573$

$\mathrm{IR}=0,599$
- Spaniomys riparius Myocastor coypus Proechimys cuvieri Thrichomys apedonte Echimys chrysurus Chakalas chrysurus Protadelphomys latus Euryzygomatomys spinosus

Mesocapromys/Mysateles sp.

- Xenodontomys simpsoni-elongatus Ctenomys boliviensis Neophanomys biplicatus

Pithanotomys columnaris

Octodon degus

Spalacopus cyanus Abrocoma bennetti

Prostichomys bowni

Stichomys regularis

Prospaniomys priscus-cf. priscus

Dudumus ruigomezi

Pampamys emmonsae

Caviocricetus lucasi

Plesiacarechimys koenigwaldi

- Acaremys murinus

Sciamys principalis

Galileomys eurygnathus

Platypittamys brachyodo

Deseadomys arambourgi

Willidewu esteparius

Sallewu esteparius

Draconomys verai

Leucokephalos zeffia

Leucokephalos zeffia

Chinchilla lanigera

Neopiblema ambrosettianus-horridula

Lagostomus maximus

Drytomomys aequatorialis

Potamarchus murinus

Dinomys branickil

Scleromys quadrangulatus

Scleromys angustus

Scleromys osbornianus

Eoviscaccia frassinetti

Eoviscaccia australis

Incamys bolivianus

Microscleromys paradoxalis-cribriphilus

Scleromys praecursor

Eoincamys pascuali

Chambiramys shipiborum

Eoincamys parvus

Chambiramys sylvaticus

Eoincamys valverdei

Eoincamys ameghino

Mayomys confluens

Eosallamys paulacoutoi-simpsoni

Dolichotis patagonum

Hydrochoerus hydrochaeris

Cavia aperea

Galea spixii

Asteromys punctus

Luantus propheticus

Australoprocta fleagi

Neoreomys australis

Branisamys luribayensis

Dasyprocta sp.

Branisamyopsis australis-praesigmoide

Neosteiromys pattoni

Eosteiromys homogeniden

Erethizon dorsatum

Coendou prehensilis-insidiosus

Steiromys detentus

Protosteiromys medianus

Hypsosteiromys axiculus

Palaeosteiromys amazonensis

Eobranisamys romeropittmana

Eobranisamys javierpradoi

Shapajamys labocensis

Tarapotomys mayoensis

Pozomys ucayaliensis

Cachiyacuy contamanensis

Eoespina woodi-Eosachacui lavocat

Canaanimys maquiensis

Cachiyacuy kumme

Thryonomys swinderianus

Paraphiomys pigotti

Metaphiomys sigotti

Acritophiomys bowni

Acritophiomys bowni

Hodsahibia azrae

"Waslamys" attiai

Ottomania proavita

Baluchimys barryi

Chapattimys wilson

Tamquammys dispinorum-wilsoni-tantillus

Reithroparamys delicatissimus

Birbalomys sondaari-woodi

Archetypomys erlianensis

cocomys ligchaensis 
ANNEXE 5. - Donnée électronique supplémentaire 1: Liste des apomorphies aux nœuds et liste des changements pour chaque caractère du consensus strict de l'AG1. Permalien vers la ressource: $\mathrm{http}: / /$ sciencepress.mnhn.fr/sites/default/files/documents/fr/g2019v41a4_1.pdf Digital Object Identifier (DOI) de ce document: https://doi.org/10.5252/geodiversitas2019v41a4_1

ANNEXE 6. - Liste des taxons cités dans l'article, avec leur(s) auteur(s) correspondant(s).

Abrocoma Waterhouse, 1837

Abrocoma bennettii Waterhouse, 1837

ACAREMYIDAE Wood, 1949

Acaremys murinus Ameghino, 1887

Acritophiomys bowni Sallam, Seiffert \& Simons, 2012

AdelPhOMYIDAe Patterson \& Pascual, 1968, n. stat.

Adelphomys candidus Ameghino, 1887

Archetypomys erlianensis Meng, Li, Ni, Wang \& Beard, 2007

Asteromys punctus Wood \& Patterson, 1959

Asteromys bolivianus (Lavocat, 1976)

Australoprocta fleagi Kramarz, 1998

Baluchimys barryi Flynn, Jacobs \& Cheema, 1986

Birbalomys sondaari Hussain, de Bruijn \& Leinders, 1978

Birbalomys woodi Sahni \& Khare, 1973

Branisamyopsis australis Candela, 2003

Branisamyopsis praesigmoides Candela, 2003

Branisamys luribayensis Hoffstetter \& Lavocat, 1970

Bugtimys zafarullahi Marivaux, Vianey-Liaud, Welcomme \& Jaeger, 2002

Cachiyacuy contamanensis Antoine, Marivaux, Croft, Billet, Ganerød, Jaramillo, Martin, Orliac, Tejada-Lara, Altamirano, Duranthon, Fanjat, Rousse \& Salas-Gismondi, 2012

Cachiyacuy kummeli Antoine, Marivaux, Croft, Billet, Ganerød, Jaramillo, Martin, Orliac, Tejada-Lara, Altamirano, Duranthon, Fanjat, Rousse \& Salas-Gismondi, 2012

Canaanimys maquiensis Antoine, Marivaux, Croft, Billet, Ganerød, Jaramillo, Martin, Orliac, Tejada-Lara, Altamirano, Duranthon, Fanjat, Rousse \& Salas-Gismondi, 2012

Cavia Pallas, 1766

Cavia aperea Erxleben, 1777

CAVIIDAE Waterhouse, 1839

CAVIINAE G. Fischer, 1817

Caviocricetus lucasi Vucetich \& Verzi, 1996

CAVIOIDEA Fischer de Waldheim, 1817

Cephalomyopsis hipselodontus Vucetich, 1985

Cephalomys arcidens Wood \& Patterson, 1959

Chaetomyinae Thomas, 1897

Chaetomys Gray, 1843

Chaetomys subspinosus Olfers, 1818

Chambiramys shipiborum Boivin, 2017

Chambiramys sylvaticus Boivin, 2017

Chapattimys wilsoni Hussain, de Bruijn \& Leinders, 1978

Chinchilla Bennett, 1829

Chinchilla lanigera Bennett, 1829

Chinchillinae Bennett, 1833

Chinchilloidea Bennett, 1833

Cocomys lingchaensis Li, Zheng \& Ting, 1979

Coendou Lacépède, 1799

Coendou insidiosus (Olfers, 1818)

Coendou prehensilis (Linnaeus, 1758)
CTENOMYIDAE Lesson, 1842

Ctenomys Blainville, 1826

Ctenomys boliviensis Waterhouse, 1848

Cuniculus Brisson, 1762

Cuniculus paca (Linnaeus, 1766)

Cuniculus taczanowskii (Stolzmann, 1885)

Dasyprocta Illiger, 1811

Deseadomys arambourgi Wood \& Patterson, 1959

DinOMYIDAE Alston, 1876

Dinomys Peters, 1873

Dinomys branickii Peters, 1873

Dolichotis Desmarest, 1819

Dolichotis patagonum (Zimmermann, 1780)

Draconomys verai Vucetich, Vieytes, Pérez \& Carlini, 2010

Drytomomys aequatorialis Anthony, 1922

Drytomomys typicus (Scalabrini in Ameghino, 1889)

Dudumus ruigomezi Arnal, Kramarz, Vucetich \& Vieytes, 2014

ECHIMYIDAE Gray, 1825

ECHIMYINAE Gray, 1825

ECHIMYINI Fabre, Upham, Emmons, Justy, Leite, Loss, Orlando,

Tilak, Patterson \& Douzery, 2017

Echimys Cuvier, 1809

Echimys chrysurus (Zimmermann, 1780)

Eobranisamys javierpradoi Boivin, 2017

Eobranisamys romeropittmanae Frailey \& Campbell, 2004

Eoespina woodi Frailey \& Campbell, 2004

Eoincamys ameghinoi Frailey \& Campbell, 2004

Eoincamys parvus Boivin, Marivaux, Pujos, Salas-Gismondi,

Tejada-Lara, Varas-Malca \& Antoine, 2018

Eoincamys pascuali Frailey \& Campbell, 2004

Eoincamys valverdei Boivin, Marivaux, Pujos, Salas-Gismondi,

Tejada-Lara, Varas-Malca \& Antoine, 2018

Eosachacui lavocati Frailey \& Campbell, 2004

Eosallamys paulacoutoi Frailey \& Campbell, 2004

Eosallamys simpsoni Frailey \& Campbell, 2004

Eosteiromys homogenidens Ameghino, 1902

Eoviscaccia australis Vucetich, 1989

Eoviscaccia boliviana Vucetich, 1989

Eoviscaccia frassinettii Bertrand, Flynn, Croft \& Wyss, 2012

ERETHICAVIOI Boivin, n. taxon

Erethizon Cuvier, 1823

Erethizon dorsatum (Linnaeus, 1758)

Erethizon insidiosus (Olfers, 1818)

Erethizon prehensilis (Linnaeus, 1758)

ERETHIZONTINAE Bonaparte, 1845

ERETHIZONTOIDEA Bonaparte, 1845

Euryzygomatomys Goeldi, 1901

Euryzygomatomys spinosus (Fischer, 1814)

Galea Meyen, 1833

Galea spixii (Wagler, 1831) 
ANNEXE 6. - Liste des taxons cités dans l'article, avec leur auteur correspondant (suite).

Galileomys eurygnathus Kramarz, 2004

Garridomys curunuquem Kramarz, Vucetich \& Arnal, 2013

Gaudeamus aegyptius Wood, 1968

Gaudeamus aslius Sallam, Seiffert \& Simons, 2011

Gaudeamus hylaeus Sallam, Seiffert \& Simons, 2011

Gaudeamus lavocati Coster, Benammi, Lazzari, Billet, Martin,

Salem, Bilal, Chaimanee, Schuster, Valentin, Brunet \& Jaeger, 2010

Hodsahibia azrae Flynn, Jacobs \& Cheema, 1986

Hydrochoerus Brisson, 1762

Hydrochoerus hydrochaeris (Linnaeus, 1766)

Hypsosteiromys axiculus Ameghino, 1902

HYSTRICOGNATHI Tullberg, 1899

Incamys bolivianus Hoffstetter \& Lavocat, 1970

Lagidium Meyen, 1833

Lagidium viscacia (Molina, 1782)

Lagostomus Brookes, 1829

Lagostomus maximus (Desmarest, 1817)

Leucokephalos zeffiae Vucetich, Dozo, Arnal \& Pérez, 2015

Litodontomys chubutomys Wood \& Patterson, 1959

Loretomys minutus Boivin, 2017

Luantus propheticus Kramarz, 2006

Makalata Husson, 1978

Makalata armata (Geoffroy Saint-Hilaire, 1830)

Makalata didelphoides (Desmarest, 1817)

Maquiamys praecursor (Boivin, 2017), n. comb.

Maruchito trilofodonte Vucetich, Mazzoni \& Pardiñas, 1993

Mayomys confluens Boivin, Marivaux, Pujos, Salas-Gismondi,

Tejada-Lara, Varas-Malca \& Antoine, 2018

Mesocapromys Varona, 1970

Metaphiomys schaubi Wood, 1968

Microscleromys cribriphilus Walton, 1997

Microscleromys paradoxalis Walton, 1997

Myocastor Kerr, 1792

Myocastor coypus (Molina, 1782)

MYOCASTORINI Fabre, Upham, Emmons, Justy, Leite,

Loss, Orlando, Tilak, Patterson \& Douzery, 2017

Mysateles Lesson, 1842

Neoepiblema ambrosettianus (Ameghino, 1889)

Neoepiblema horridula (Ameghino, 1886)

Neophanomys biplicatus Rovereto, 1914

Neoreomys australis Ameghino, 1887

Neosteiromys pattoni Candela, 2004

OCTOCHINCHILLOI Boivin, n. taxon

Octodon Bennett, 1832

Octodon degus (Molina, 1782)

OCTODONTIDAE Waterhouse, 1839

OCTODONTOIDEA Waterhouse, 1839

Ottomania proavita de Bruijn, Ünay, Saraç \& Yïmaz, 2003

Palaeosteiromys amazonensis Boivin, 2017
Pampamys emmonsae Verzi, Vucetich \& Montalvo, 1995

Paraphiomys pigotti Andrews, 1914

Perimys intermedius Kramarz, 2002

Phiomys andrewsi Osborn, 1908

Phiomys hammudai Jaeger \& Marivaux, 2010

Phoberomys burmeisteri (Ameghino, 1886)

Phoberomys pattersoni Mones, 1980

Pithanotomys columnaris Ameghino, 1887

Platypittamys brachyodon Wood, 1949

Plesiacarechimys koenigswaldi Vucetich \& Vieytes, 2006

Plesiosteiromys newelli Boivin, 2017

Potamarchus murinus Burmeister, 1885

Pozomys ucayaliensis Boivin, 2017

Proechimys Allen, 1899

Proechimys cuvieri Petter, 1978

Prospaniomys priscus Ameghino, 1902

Prospaniomys cf. priscus Arnal \& Kramarz, 2011

Prostichomys bowni Kramarz, 2001

Protadelphomys latus Ameghino, 1902

Protosteiromys medianus Wood \& Patterson, 1959

Reithroparamys delicatissimus Leidy, 1871

Sallamys pascuali Hoffstetter \& Lavocat, 1970

Sciamys principalis Ameghino, 1887

Scleromys angustus Ameghino, 1887

Scleromys osbornianus Ameghino, 1894

Scleromys praecursor Boivin, 2017

Scleromys quadrangulatus Kramarz, 2006

Shapajamys labocensis Boivin, Marivaux, Pujos, Salas-Gismondi,

Tejada-Lara, Varas-Malca \& Antoine, 2018

Soriamys gaimanensis Kramarz, 2001

Spalacopus Wagler, 1832

Spalacopus cyanus (Molina, 1782)

Spaniomys riparius Ameghino, 1887

Steiromys detentus Ameghino, 1887

Stichomys regularis Ameghino, 1887

Tamquammys dispinorum Tong, 1997

Tamquammys wilsoni Dawson, Li \& Qi, 1984

Tamquammys tantillus Shevyreva, 1971

Tarapotomys mayoensis Boivin, Marivaux, Pujos, Salas-Gismondi, Tejada-Lara, Varas-Malca \& Antoine, 2018

Tarapotomys subandinus Boivin, Marivaux, Pujos, Salas-Gismondi, Tejada-Lara, Varas-Malca \& Antoine, 2018

Thrichomys Trouessart, 1880

Thrichomys apereoides (Lund, 1839)

Thryonomys Fitzinger, 1867

Thryonomys swinderianus (Temminck, 1827)

Tribosphenomys minutus Meng, Wyss, Dawson \& Zhai, 1994

Willidewu esteparius Vucetich \& Verzi, 1991

Xenodontomys elongatus Verzi, Montalvo \& Tiranti, 2003

Xenodontomys simpsoni Kraglievich, 1961 


\section{TABLE DES MATIÈRES}

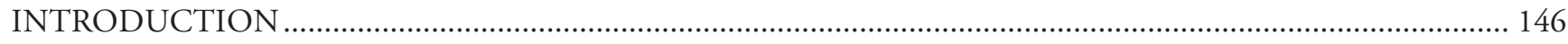

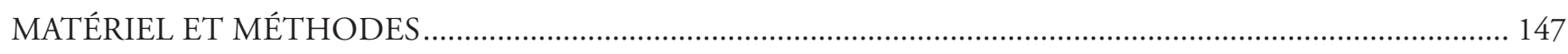

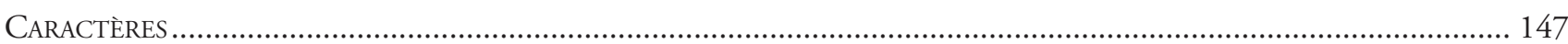

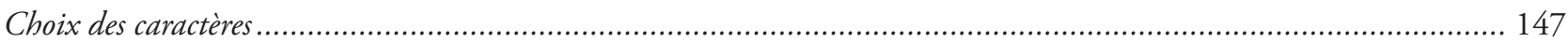

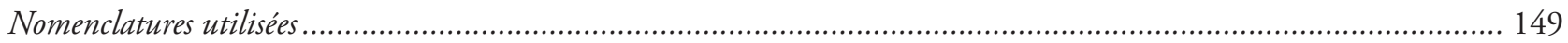

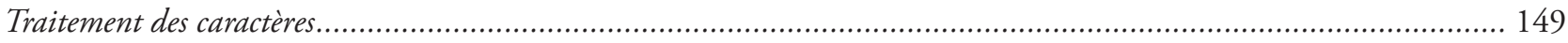

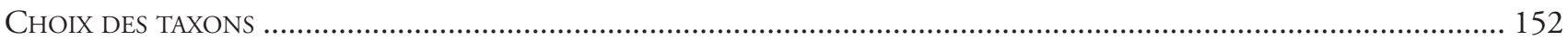

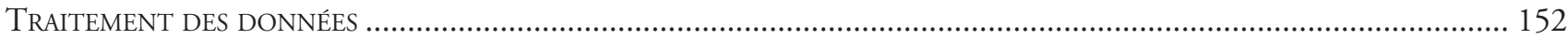

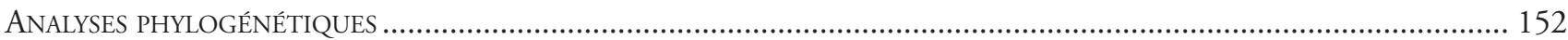

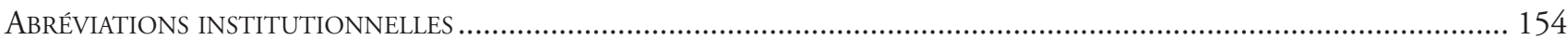

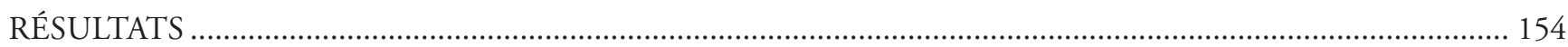

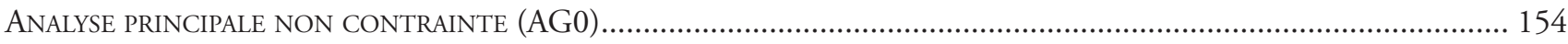

ANALYSE PRINCIPALE CONTRAINTE (AG1 ET AG1 NON ÉQUILIBRÉE) ……........................................................... 154

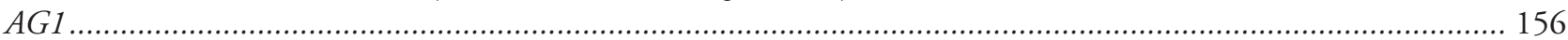

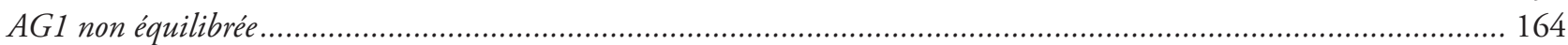

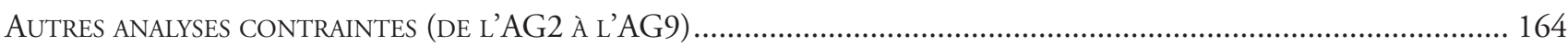

AG2: inclusion de Gaudeamus aslius, G. hylaeus et G. aegyptius ......................................................................... 164

AG3: inclusion d' 'Asteromys» bolivianus, de Cephalomys arcidens, Litodontomys et de Soriamys non contraints... 164

AG4: inclusion d' 'Asteromys» bolivianus, Asteromys punctus, de Cephalomys arcidens, Litodontomys

et de Soriamys, tous contraints en un clade divergeant au sein des chinchillö̈des .................................................... 165

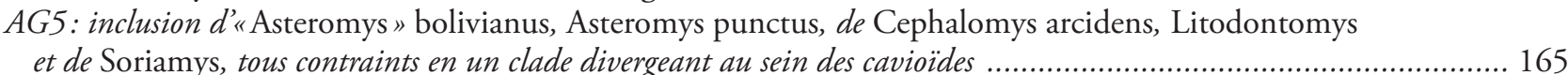

AG6: inclusion d' 'Asteromys» bolivianus, de Cephalomys arcidens et de Litodontomys, non contraints.................. 165

AG7: inclusion d'«Asteromys » bolivianus, Asteromys punctus, de Cephalomys arcidens et de Litodontomys,

tous contraints en un clade divergeant dans les chinchillö̈des............................................................................. 165

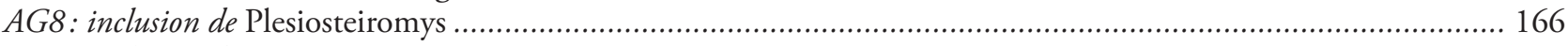

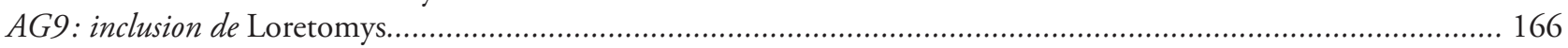

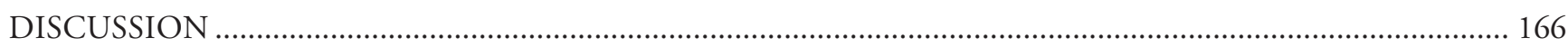

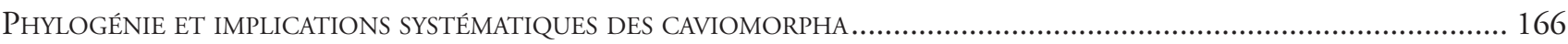

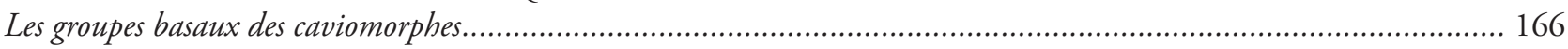

Le statut de Palaeosteiromys : éréthizontoïde? cavioide? ou autre? ...................................................................... 169

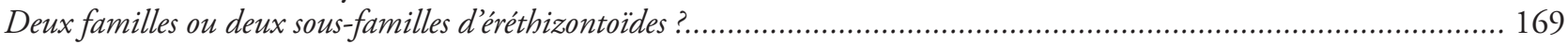

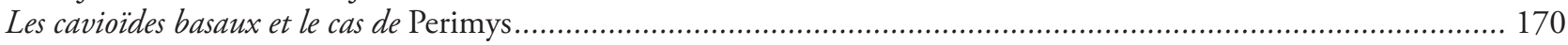

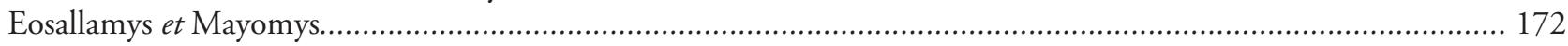

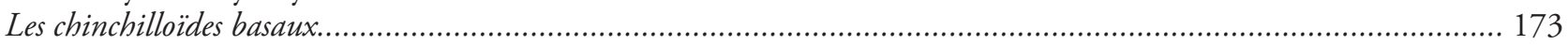

Octodontoïdes: nouvelle définition des Acaremyidae et Adelphomyidae, n. stat., et taxons fossiles au sein du groupe couronne........ 175

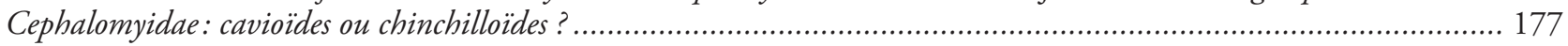

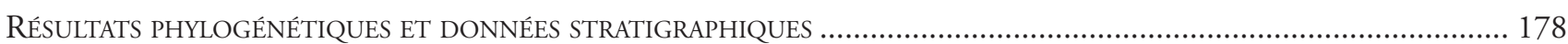

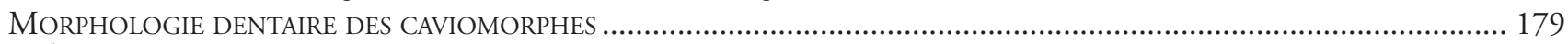

Évolution morphologique du patron dentaire des caviomorphes au Paléogène ........................................................... 179

Caractéristiques dentaires et tendances évolutives chez les quatre super-familles ........................................................ 180

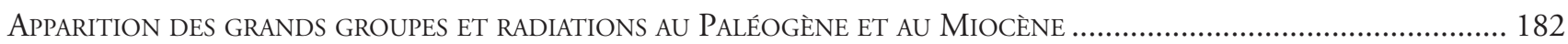

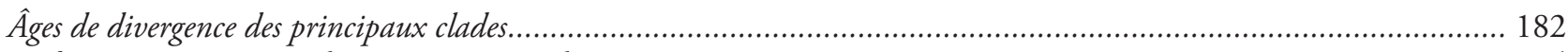

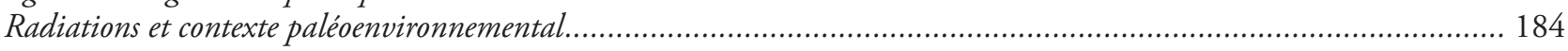

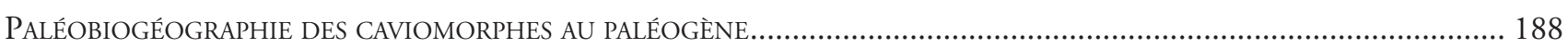

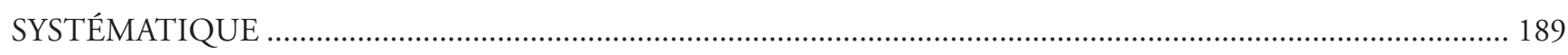

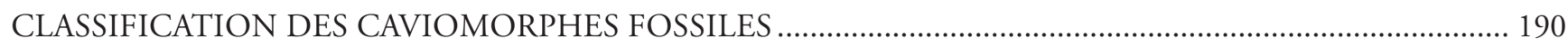

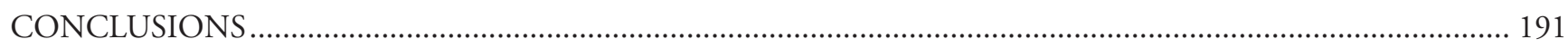

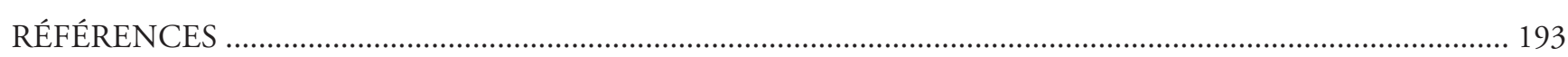

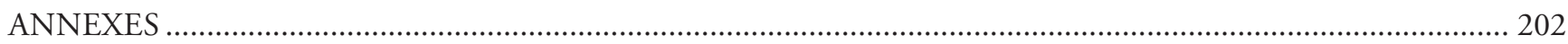

